\title{
Calculation of Graviton Scattering Amplitudes Using New Techniques
}

A thesis submitted to the University of Wales for the degree of Doctor of Philosophy.

- Paul Stephen Norridge

Department of Physics

University of Wales, Swansea

Swansea SA2 $8 P P$

$U K$

March 1996 


\section{Declaration}

This work has not previously been submitted for any degree and is not being concurrently submitted in candidature for any degree.

Signed

Date 15 th May 96

This thesis is the result of my own investigations carried out under the supervision of Dr. David Dunbar. Except where otherwise stated it is original.

Other sources are acknowledged by references to the bibliography.

Signed .

Date 1 ț..... May 96

I give consent for my thesis, if accepted, to be available for photocopying and inter-library loan, and for the title and summary to be made available to outside organisations.

Signed .

Date 15 th May 96

Material from chapters 5 and 6 has previously been published as

Calculation of Graviton Scattering Amplitudes using String-Based Methods.

David C. Dunbar and Paul S. Norridge.

Nucl. Phys. B483:181 (1995).

Material from chapter 9 and section 8.1 has been published as

Infinities in Graviton Scattering Amplitudes.

David C. Dunbar and Paul S. Norridge.

SWAT/95.59 - hep-th/9512084.

Material from sections 8.2 and 8.3 will appear in preprint

Recovering Infinities in Graviton Scattering Amplitudes using Cutkosky rules.

Paul S. Norridge.

SWAT/96-100. 


\section{Abstract}

- The calculations of scattering amplitudes in gauge theories are well-known for the difficulties which they present. Traditional methods for obtaining perturbative results (e.g., Feynman diagrams) are often impractical in all but the simplest cases. This has led to searches for new techniques which simplify the calculational process and allow a wider set of results to be obtained. In recent - years these searches have produced a number of new methods which have been used successfully to find many amplitudes in QCD.

Scattering amplitudes in perturbative quantum gravity are among the most difficult to calculate. The success of the new techniques in the standard model scenario suggests that it would be profitable to look at extensions of these to PQG. Here we look at the ways in which two of these methods - the stringinspired and Cutkosky rules - can be applied in the new situation. We show how a set of string-inspired rules for one-loop graviton amplitudes can be derived by looking at a closed bosonic string and how we can use information provided by the Cutkosky method. As an example of the string-inspired technique we calculate all one-loop four graviton amplitudes with massless internal particles. An application of the Cutkosky rules allows us to check these results as well as to obtain information about divergences in PQG theories. A combination of the Cutkosky rules and dimensional reduction enables us to re-derive some of the string-based results and to extend these to cases with massive internal particles. 
To my family.

weavers' fingers flying on the loom patterns shift too fast to be discerned all these years of thinking ended up like this in front of all this beauty understanding nothing

Bruce Cockburn 


\section{Acknowledgements}

'Thanks are due to...

Dave Dunbar for his excellent supervision;

My family for their continual support;

The Swansea Theory Group Postgraduates for discussions and distractions, and especially Doug and Rhys for their proof-reading;

Zvi Bern, Lance Dixon, Andrew Morgan and Graham Shore

. for conversations and vital snippets of information;

The UCLA physics department for their hospitality during some of this work;

My friends at New Covenant Church Swansea, especially the Williams' - Mark, Sue, Sam, David, Thomas and Hannah - and the Griffiths' - Hugh, Jayne and Nathan for keeping me housed and sane at various points;

Neil Townsend for further proof-reading and sanity via email;

P.P.A.R.C. (né S.E.R.C.) for the financial support of this work;

and God, for creating such a beautiful and interesting universe. 


\section{Contents}

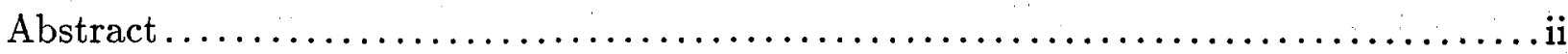

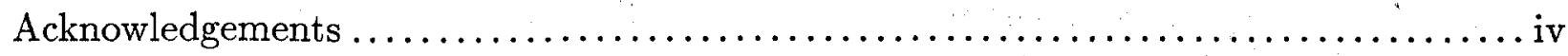

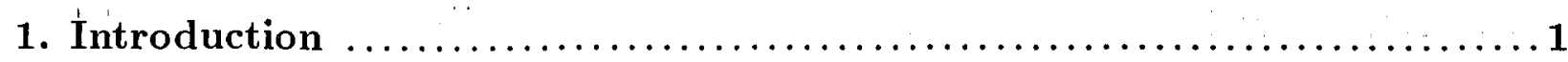

2. Gravity in Quantum Field Theory $\ldots \ldots \ldots \ldots \ldots \ldots \ldots \ldots \ldots \ldots \ldots \ldots$

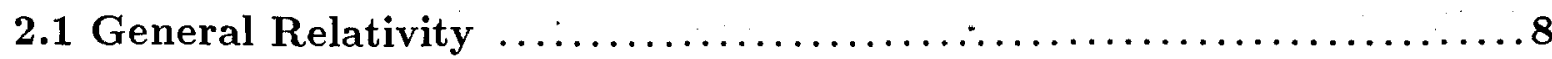

2.2 Particle Physics Approaches to Quantum Gravity ............ 10

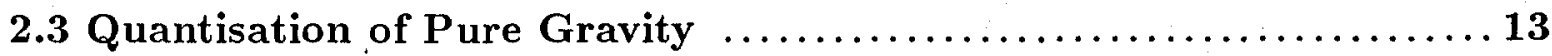

2.4 Coupling to Other Particles: Bosons $\ldots \ldots \ldots \ldots \ldots \ldots \ldots \ldots \ldots \ldots \ldots$

2.5 Coupling to Other Particles: Fermions $\ldots \ldots \ldots \ldots \ldots \ldots \ldots \ldots \ldots \ldots$

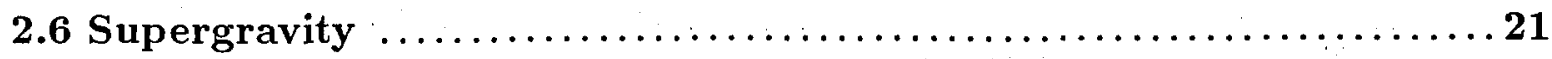

2.7 Previous Calculations in Perturbative Quantum Gravity ..........24

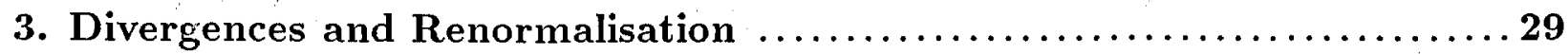

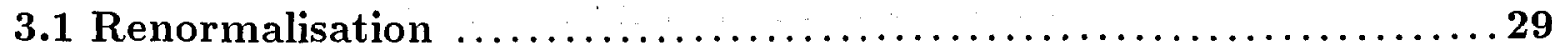

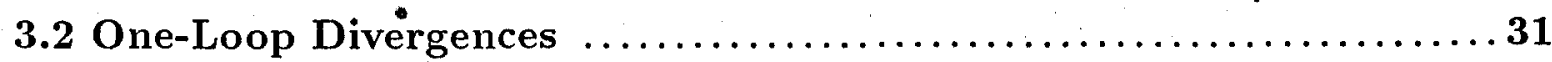

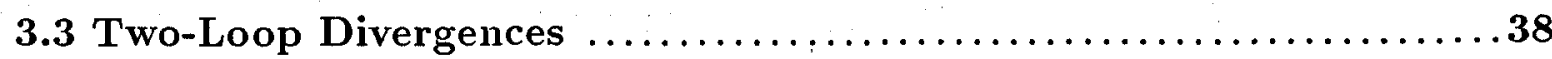

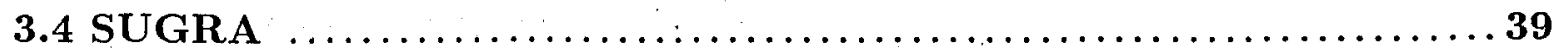

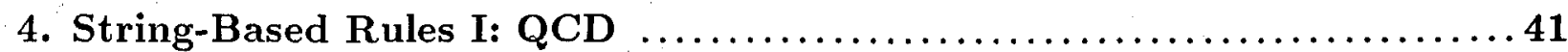

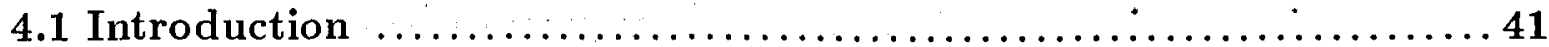

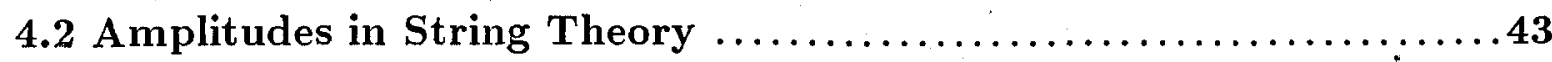

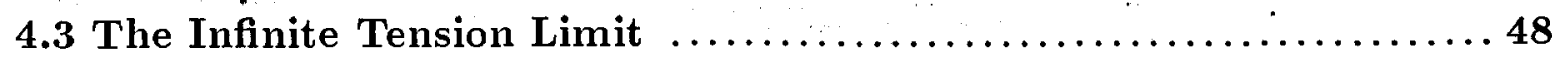

4.4 Derivation of QCD Rules from Open Strings $\ldots \ldots \ldots \ldots \ldots \ldots \ldots \ldots$

4.5 QCD Rules $\ldots \ldots \ldots \ldots \ldots \ldots \ldots \ldots \ldots \ldots \ldots \ldots \ldots \ldots \ldots \ldots \ldots \ldots$

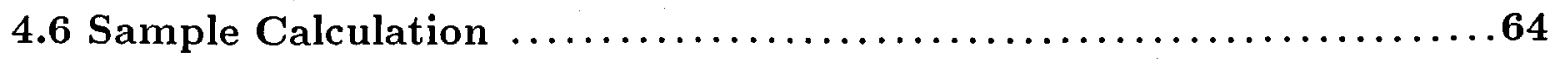




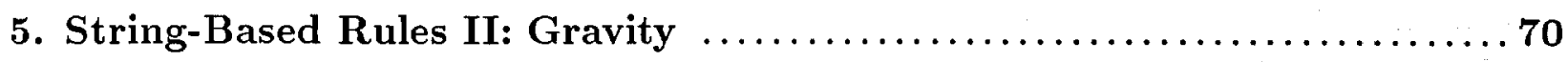

5.1 Derivation of Gravity Rules from Closed Strings ...............71

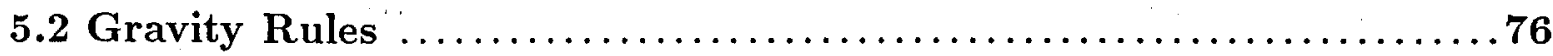

5.3 Sample Calculation $\ldots \ldots \ldots \ldots \ldots \ldots \ldots \ldots \ldots \ldots \ldots \ldots \ldots \ldots \ldots \ldots$.

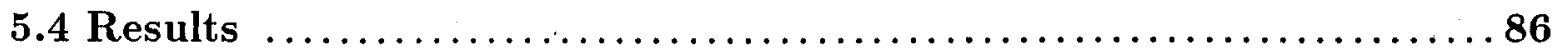

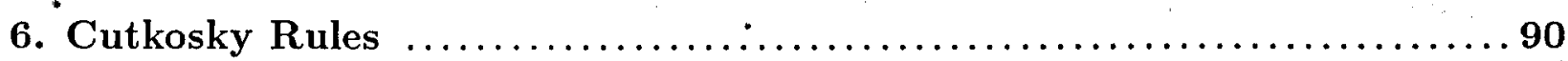

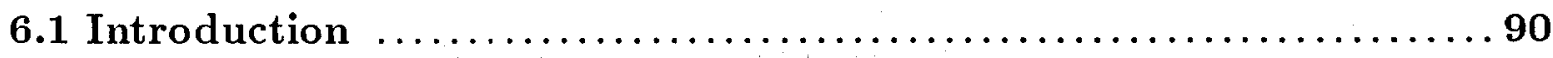

6.2 Application to One-Loop Calculations $\ldots \ldots \ldots \ldots \ldots \ldots \ldots \ldots \ldots . \ldots 2$

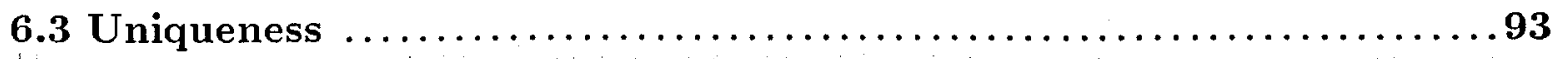

6.4 External Graviton Calculations $\ldots \ldots \ldots \ldots \ldots \ldots \ldots \ldots \ldots \ldots \ldots \ldots \ldots$

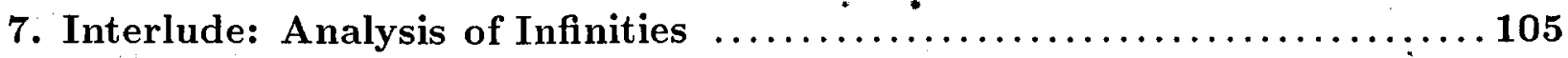

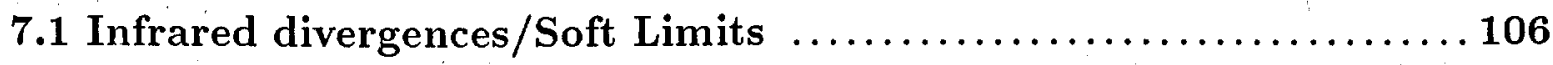

7.2 One-loop Amplitudes with External Gravitons $\ldots \ldots \ldots \ldots \ldots \ldots 113$

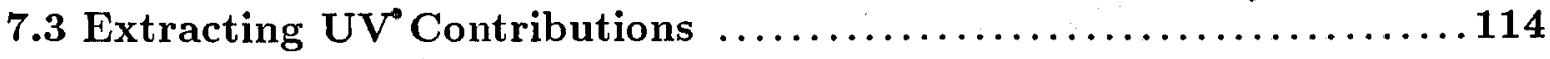

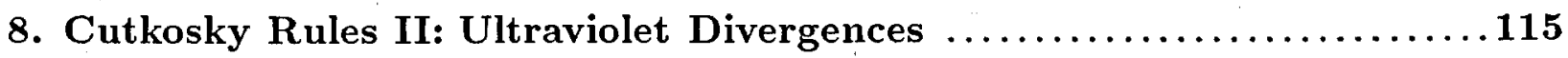

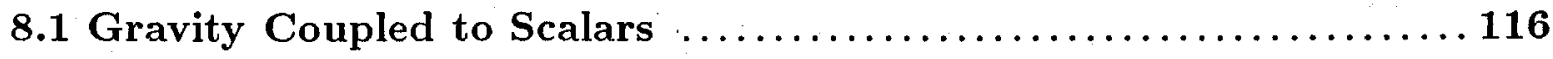

8.2 Gravity Coupled to Fermions ........................... 125

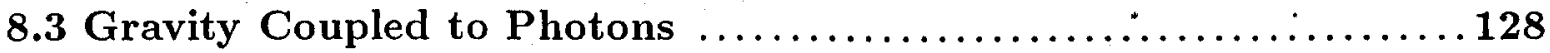

9. Cutkosky Rules III: Complete Amplitudes .....................134

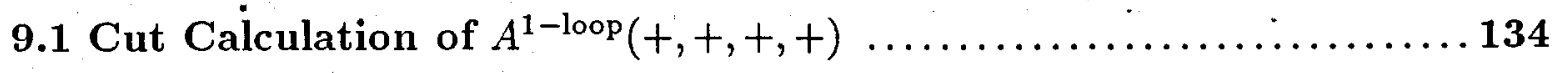

9.2 Loops with Internal Massive Particles $\ldots \ldots \ldots \ldots \ldots \ldots \ldots \ldots \ldots \ldots 138$

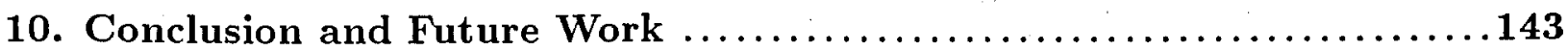




\section{Appendices}

I. Quantities in General Relativity $\ldots \ldots \ldots \ldots \ldots \ldots \ldots \ldots \ldots \ldots \ldots \ldots \ldots \ldots$

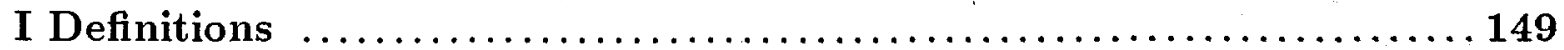

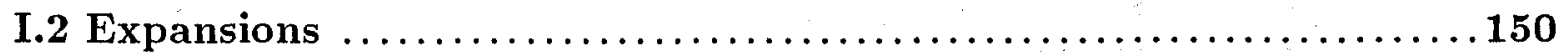

II. Expansions of String Theory Green Functions $\ldots \ldots \ldots \ldots \ldots \ldots \ldots \ldots \ldots$

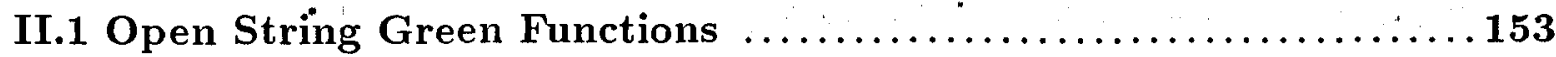

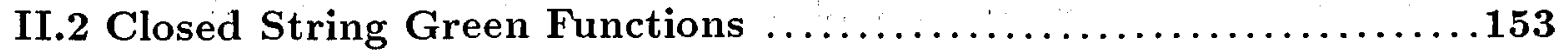

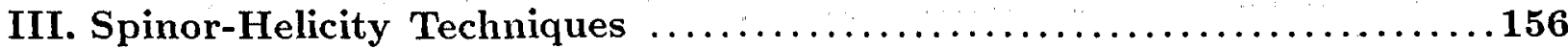

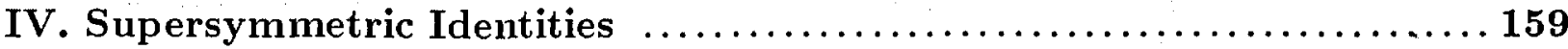

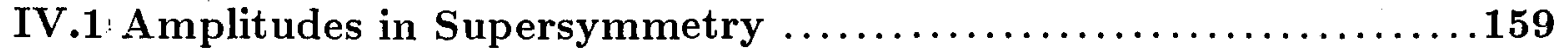

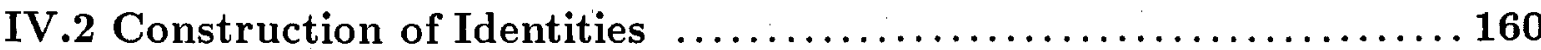

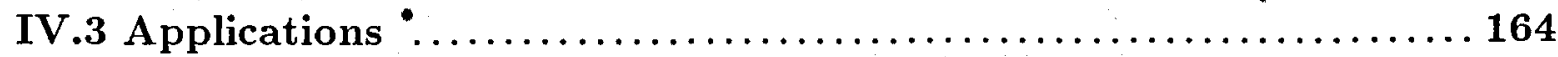

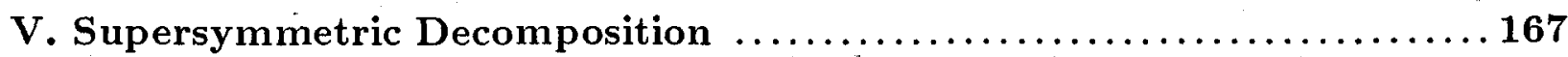

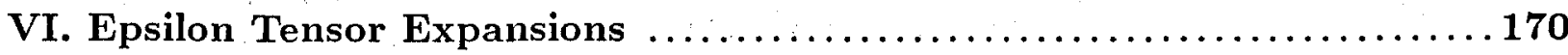

References 


\section{Introduction}

The ability to carry out calculations is an important part of physics. Whether we are interested in the formal aspects of a theory or its value as a model, calculations will be a prime source of information. Moreover, if we cannot use a theory to produce quantitative predictions then it will be practically useless. Consequently, the development of efficient calculational methods is vital for the continuing progress and serviceability of physics.

In quantum field theories (QFTs) the calculation of physical quantities is often a difficult process. A large proportion of results cannot be obtained precisely, so we rely on perturbative expansions to give us some approximation. These are expressed as a series in some small parameter of the theory. Rather than looking for the whole result, we try to find terms in this series. The conventional way for finding terms in such an expansion is the well- . known 'Feynman Diagram' method [1]. This involves drawing graphical representations of all processes - constructed from 'propagators' and 'vertices'. Each vertex and propagator is related to an algebraic expression, derived from the Lagrangian of the theory. These relations allow us to construct an integral expression for each diagram. We can class the integrals according to perturbative order. The sum of all the integrals in a particular class gives us the required term in the expansion.

In principle, Feynman rules are applicable for all orders in the perturbative expansion. However, it will be clear to anyone who has attempted to use them for gauge theory calculations that they have severe limitations. In all but the simplest cases the method proves to be prohibitively complex; the combination of a large number of diagrams and difficult algebra often make the computation of any results beyond leading order (tree 
level) impractical. We might accept this as an inherent difficulty with the theory if the resulting expressions for amplitudes were equally complex - a result of many pages length might be expected to require pages of calculation. In practice; this does not seem to be the case. There are many cancellations in the final sum of integrals, so that, while calculations can be very long, they typically produce results which can be written compactly. It seems that Feynman diagram techniques are a particularly inefficient way to carry out amplitude calculations.

This inefficiency is a severe drawback for particle physics. In quantum chromodynamics (QCD) especially, higher order corrections are important in comparisons with experiment and the lack of.results has left large theoretical uncertainties. In recent years, these problems have led to a search for alternatives to Feynman's method; techniques which produce results by a more efficient means. At tree level there have been developments such as the spinor helicity method, colour decomposition and supersymmetric (SUSY) Ward Identities (see, e.g., the review in ref. [2]). In this work we will be concerned with methods for one-loop calculations (Next to Leading Order, or NLO). In QCD, two techniques have been particularly effective at this order - the string-inspired rules [3,4] and an investigation of unitarity constraints [5]. (Though we will not discuss them here, we should also mention other developments - the worldline formalism (e.g., [6,7]), Mahlon's recursive methods [8] and the investigation of collinear limits $[9,10,11]$ - which have proved useful.)

The string inspired technique was first developed by Bern and Kosower [3]. The esserce of the method is the observation that string theories contain gauge theories in their infinite tension limit $[12,13]$. By choosing a suitable string and looking at this limit, it is possible to derive an alternative set of rules for amplitude calculations. These rules inherit many of the simplicities of one-loop string calculations. In particular, they seem to reorganise the contributions so that many cancellations occur early in the calculation, rather than in the final stages. Consequently, the amount of computation required is significantly less than with Feynman diagrams. In fact, the method is quite a contrast to 
Feynman's. It begins with a kinematic expression - the 'Master Formula' - which contains all the information for the amplitude. The rules are simply manipulations which allow results to be extracted from this. The exact forms of the 'Master Formula' and the rules depend on the initial string theory. Though the original manifestation was developed from a fully consistent heterotic string [3], it was later shown that an alternative version could be obtained by analysis of the simpler open bosonic string $[14,15]$. This, in turn, produces a simpler Master Formula and set of rules.

The second technique takes a completely different approach to the 'direct calculations' of both the Feynman and string-inspired rules. Instead, it focusses on the mathematical restrictions which can be placed on amplitudes. With the information which these provide, some attempt can be made to reconstruct expressions for the results. In this case, the mathematical restrictions are found using the Cutkosky rules $[5,16,1]$. These rules allow us to find discontinuities of an amplitude by looking at integrals involving amplitudes of lower order. If we can relate these discontinuities to other terms which might appear, then we can begin to reconstruct the full expression. So, for instance, we can determine the absorptive parts of a one-loop amplitude from our knowledge of tree results $[17,10]$. From this we can deduce which logarithmic terms must be present and place strong constraints on the total form. This provides a good check on calculations by other methods but, generally, ambiguities prevent us from determining the complete result.

The advantages of this Cutkosky-based technique are clear - since it can be used purely at the amplitude level, it circumvents many of the problems associated with Feynman diagrams; trees can be simplified before the calculation and late cancellations are reduced. The limitation of the method seems to be the ambiguities in the results. However, there are two ways in which this limitation can be overcome: First, looking at the basis of functions required to construct one-loop amplitudes, it becomes clear that many SUSY results are uniquely specified by this method [17]; the combinations of functions which cause problems are not relevant in these cases. Second, all results can be found precisely if the method is 
carried out in a dimensional regularisation scenario (i.e. $4-2 \epsilon$ dimensions) [18]; analysis of the basis functions implies that knowledge of the discontinuities at $\mathcal{O}(\epsilon)$ gives the complete amplitude at $\mathcal{O}\left(\epsilon^{0}\right)$. By using the Cutkosky-based approach in these ways, we can go beyond merely checking previous calculations and find new results.

The two one-loop techniques also allow us to incorporate other simplifications. For example, the tree-level techniques mentioned above can be used to great effect. The spinor helicity method $[19,20,21]$ expresses helicity vectors as products of Weyl spinors. In this form many inner products involving these vectors vanish. In addition, expressions for amplitudes can be written in a particularly compact form. Using this method, the 'Master Formula' in the string-inspired rules and the trees in the Cutkosky method can both be simplified greatly. The SUSY Ward Identities $[22,23,2]$ are also useful in applications of the Cutkosky rules. In SUSY theories, it is possible to relate amplitudes with different external particles. At tree level, the difference between SUSY and non-SUSY amplitudes is largely cosmetic, so we can use these SUSY relations in a more general context. As tree amplitudes are the basic building block in the Cutkosky-based one-loop calculations, these relations are a useful tool for simplifying the process.

A further application of SUSY which comes in at the one-loop level is the supersymmetric decomposition [15,24]. This is another technique inspired by string theory. It suggests that the most efficient way to calculate a complete set of amplitudes is to concentrate on those for SUSY multiplets and then to reconstruct others with linear combinations of these. We have already mentioned that considering SUSY multiplets in Cutkosky calculations is one of the ways of finding complete amplitudes. In addition, for both the string-based and Cutkosky methods these results involve the simplest calculations.

The combination of the string-inspired rules and the Cutkosky-based method (together with the SUSY and spinor helicity simplifications) have led to a range of new results. In QCD, they have produced all one-loop four- and five-gluon amplitudes $[3,4,25]$ and oneloop $\mathrm{n}$-gluon amplitudes with specific helicity configurations $[10,17,26]$. They have also 
contributed to the calculation of QCD amplitudes with two external quarks [27] and some electroweak results [24].

Even within QFTs, quantum theories of gravity - for instance perturbative quantum gravity (PQG) $[28,29]$ and supergravity (SUGRA) [30] - are renowned for the calculational difficulties they present. The large number of different processes and the algebraic complexity of contributions conspire to make the majority of calculations practically impossible. A comparison with QCD shows the problems clearly: The Feynman rules for PQG include more vertices than $\mathrm{QCD}$ and the vertex formulae are more complex. In addition, PQG integrals typically involve more powers of loop momenta. Clearly, quantum gravity amplitudes will be harder to obtain than the (already difficult) QCD equivalents. As before, we can contrast the computational difficulties with the relatively simple results found for typical amplitudes.

Given the success of the new techniques for QCD, we might wonder if we can use similar methods for quantum gravity. This is the question which will be addressed in this thesis. We will take the work outlined above and look at the ways it can be adapted for . PQG and SUGRA calculations. We will see that both string-based and Cutkosky-based methods can be used successfully in this new situation. As in the QCD case, these two methods will also allow us to use spinor helicity and SUSY methods to simplify amplitudes further and calculate more efficiently.

The development of string-inspired rules for gravity closely resembles the QCD case. Again, we find a relevant string theory and look at the infinite-tension limit [31]. Again, we find a 'Master Formula' and a set of rules which can be used to extract amplitude expressions.' The primary difference between the two cases is the starting point; open bosonic strings do not include the spin-2 graviton required by PQG. Instead, we must look at a closed bosonic string [12]. While the derivations in these two cases are similar, we find the closed string case includes some complications: The closed string has two sets 
of variables - left- and right-moving - where the open string has one. Consequently, the string expression will include components of both sets, as well as interactions between them. Thus, we expect the string-based rules for gravity to resemble, but differ from, those for QCD. •

The Cutkosky rules can be used in precisely the same way for any theory, so we do not need to make changes for gravity; the difference will be the amplitudes which we use as building blocks. We will look here at analogous exercises to those carried out in QCD - the reconstruction of one-loop amplitudes from trees. As in QCD, we can use this to find some SUSY amplitudes exactly, though the number in gravity is reduced significantly. In gravity, the method is useful cven when we cannot find complete amplitudes; the Cutkosky rules can be useful for investigating the problematic divergences which plague these theories. If we are interested in obtaining a wider range of complete amplitudes then we can again turn to the combination and dimensional regularisation.

As examples of these methods we will look at the calculation of one-loop amplitudes with external gravitons. We will also use the Cutkosky rules to investigate some of the one-loop divergences in PQG.

The work presented here is structured as follows: In the next chapter we outline the conventional construction of perturbative quantum gravity and give a brief discussion of supersymmetry and supergravity theories. Since the major problems in quantum gravity are related to divergences and renormalisability, we go on to look at these in chapter 3; calculations later will help to confirm or extend existing results in this area. In chapter 4 we review the string-inspired technique for $\mathrm{QCD}$ and include a sample calculation. This introduces, in a simpler case, many of the ideas required in the extension to gravity. We discuss this extension in chapter 5 . Using the string-inspired rules we are able to calculate all the one-loop four graviton amplitudes; that is, all amplitudes with four external gravitons and any choice of internal (massless) particles. 
In chapter 6 we introduce the Cutkosky rules. We use these as check on the amplitudes which we have calculated by the string-inspired techniques. We follow this with a short interlude discussing how to find infrared divergences in one-loop quantum gravity amplitudes. We can use this to confirm that only IR divergences occur in the one-loop graviton amplitudes but, more importantly, it prepares us for chapter 8 in which we calculate divergences in a wider range of gravity amplitudes using the Cutkosky techniques. Since we can identify IR divergences independently, we can use these results to find UV divergences in quantum gravity. We use these to confirm earlier derivations of counterterms and to obtain new information about those previously unknown.

We end our calculations in chapter 9, where we look at the extension of the Cutkosky technique commented on above. Combining Cutkosky rules with an explicit use of dimensional regularisation gives us the potential to recover any one-loop amplitude efficiently. As an example of this we re-derive one of the earlier results found with the string-inspired rules. We also find an expression for a four-graviton amplitude with internal massive particles. "

After a concluding chapter, we end with a series of appendices which describe some of the less important techniques which we implemented in the preceeding calculations (SUSY Ward identities, SUSY decomposition and Spinor Helicity methods), as well as outlining some of the more technical aspects of the work. 
We know that we live in contradiction, but that we must refuse this contradiction and do what is needed to reduce it.

Albert Camus

\section{Gravity in Quantum Field Theory}

Gravity has long posed a problem for particle physicists. While we have a successful classical theory in General Relativity (GR), this does not fit with our quantum theories of matter in a neat 'philosophical' way. This is a puzzle which cannot easily be side-stepped since matter is related directly to GR quantities in Einstein's field equations (2.9). So, we would like to find some consistent method to link GR to quantum field theories, preferably by quantising gravity itself. The difficulty is that it is not clear what form such a quantum theory of gravity should take - all those proposed have flaws of some sort. A continuing problem in all investigations in this area is the difficulty of calculating quantities, as we discussed in the Introduction. In particular, this has made hard work of attempts to . understand the formal problems such as renormalisation (see next chapter). Hopefully, some of these restrictions will be relieved by the work presented here.

Before we look at the new calculational techniques, let us sketch the scenario in which we will be working. We will begin in the next section by giving a brief outline of GR. Following this, we will go on to discuss some of the conjectures for describing gravity in a way consistent with theories of matter. We will then look in detail at those to which our calculations will apply - the Lagrangian-based theories. This will involve short descriptions of the approach which we take with the pure gravity Lagrangian, the ways in which bosons and fermions are coupled to gravity and extensions to SUGRA. Finally, we will give an overview of the previous calculations which have been carried out. 


\subsection{General Relativity}

Let us begin with a very brief overview of the essential aspects of General Relativity to establish notation (for all full discussion see, e.g., [32]). GR is an extension of Special Relativity (SR) which includes gravity. To begin with, the Lorentz transformation of SR is replaced by a general coordinate (gauge) transformation:

$$
\begin{aligned}
x^{\mu} & \rightarrow x^{\prime \mu}(x) \\
\mathrm{d} x^{\mu} & \rightarrow \mathrm{d} x^{\prime \mu}=\Lambda_{\nu}^{\mu} \mathrm{d} x^{\prime \nu}
\end{aligned}
$$

(That is, transformations under the group $G L(4, \mathcal{R})$.) Scalars, vectors and tensors in the theory are then quantities which transform under the gauge transformations as

$$
\begin{array}{r}
\phi(x) \rightarrow \phi^{\prime}\left(x^{\prime}\right)=\phi(x) \\
A_{\mu}(x) \rightarrow A_{\mu}^{\prime}\left(x^{\prime}\right)=\left(\Lambda^{-1}\right)_{\mu}^{\nu} A_{\nu}(x) \\
T_{\nu_{1} \ldots \nu_{m}}^{\mu_{1} \ldots \mu_{n}}(x) \rightarrow T_{\nu_{1} \ldots \nu_{m}}^{\mu_{1} \ldots \mu_{n}}\left(x^{\prime}\right)=\Lambda_{\rho_{1}}^{\mu_{1}} \cdots \Lambda_{\rho_{n}}^{\mu_{n}} \\
\\
\times\left(\Lambda^{-1}\right)_{\nu_{1}}^{\sigma_{1}} \ldots\left(\Lambda^{-1}\right)_{\nu_{m}}^{\sigma_{m}} T_{\sigma_{1} \ldots \sigma_{m}}^{\rho_{1} \ldots \rho_{n}}(x) .
\end{array}
$$

Gravity comes into the theory as a result of the curvature of space-time. This is encoded in the metric tensor, $g_{\mu \nu}$ (together with an inverse $g^{\mu \nu}$ s.t. $g^{\mu \alpha} g_{\alpha \nu}=\delta_{\nu}^{\mu}$ ). The curvature is then seen in a typical line element, defined as

$$
\mathrm{d} \tau^{2}=g_{\mu \nu} \mathrm{d} x^{\mu} \mathrm{d} x^{\nu}
$$

The metric is used to raise, lower and contract indices on tensors (e.g., $A_{\mu}=g_{\mu \nu} A^{\nu}$ ).

As with all gauge theories, we must define a covariant derivative. This is the conventional derivative plus extra terms which produce the correct transformation properties. For example, for scalars and vectors we define it to be

$$
\begin{aligned}
\nabla_{\mu} \phi & =\partial_{\mu} \phi \\
\nabla_{\mu} A_{\nu} & =A_{\nu ; \mu}=\partial_{\mu} A_{\nu}-\Gamma_{\mu \nu}^{\lambda} A_{\lambda}
\end{aligned}
$$

(For a more general expression, see appendix I.) $\Gamma$ is the connection, which is defined in such way as to give the correct transformation properties to $\nabla_{\mu}$. In GR we assume that 
the theory is 'torsion-free', that is, that $\Gamma_{\mu \nu}^{\lambda}=\Gamma_{\nu \mu}^{\lambda}$. The usual choice for $\Gamma$ is

$$
\Gamma_{\mu \nu}^{\lambda}=\frac{1}{2} g^{\lambda \alpha}\left(\partial_{\mu} g_{\alpha \nu}+\partial_{\nu} g_{\alpha \mu}-\partial_{\alpha} g_{\mu \nu}\right)
$$

This is the 'metric connection' and, in this case, $\Gamma$ is referred to as the Christoffel symbol. This choice is made so that

$$
\nabla_{\lambda} g_{\mu \nu}=0
$$

- The other quantities which we define are curvature tensors. These give a guide to the geometry of the space we are working in. The most usual choices are the Riemannian tensor

$$
\cdot R_{\nu \sigma \rho}^{\mu}=\partial_{\sigma} \Gamma_{\nu \rho}^{\mu}-\partial_{\rho} \Gamma_{\nu \sigma}^{\mu}+\Gamma_{\nu \sigma}^{\alpha} \Gamma_{\alpha \rho}^{\mu}-\dot{\Gamma}_{\nu \rho}^{\alpha} \Gamma_{\alpha \sigma}^{\mu}
$$

and the Ricci tensor and scalar

$$
\begin{aligned}
R_{\mu \nu} & =R_{\mu \alpha \nu}^{\alpha} \\
R & =g^{\mu \nu} R_{\mu \nu \cdot *}
\end{aligned}
$$

The dependence of the curvature on the matter in the universe is expressed in the Einstein field equations:

$$
R_{\mu \nu}-\frac{1}{2} g_{\mu \nu} R=8 \pi G T_{\mu \nu}
$$

$T_{\mu \nu}$ is the energy-momentum tensor of the matter. Clearly, this is a non-linear equation in $g_{\mu \nu}$, making explicit our contention that this is what encodes the curvature.

\subsection{Particle Physics Approaches to Quantum Gravity}

There have been a number of attempts to include gravity in a quantum theory; all have problems of some sort. The theories which we will discuss here are perturbative quantum gravity (PQG) $[28,29,33]$, supergravity (SUGRA) [30] and string theory (ST) [34]. We will look at string theory later; this forms the basis for one of the new calculational techniques. String theories include gravity with matter in a way which avoids the theoretical problems present in other models. However, they have their own difficulties: At present it is not clear how to construct a ST which fits with experimental results. In this chapter we give 
an overview of PQG and SUGRA. It is these theories at which our calculations will be aimed.

PQG takes the most obvious path to a quantum theory of gravity: A Lagrangian which leads to the Einstein field equations, or some generalisation of these. The Lagrangian which gives the left hand side of (2.9) exactly is the Einstein-Hilbert action,

$$
\mathcal{L}=-\frac{2}{\kappa^{2}} \sqrt{-g} R\left(g_{\mu \nu}\right)
$$

(This is the case for a theory with no matter; $T_{\mu \nu}=0$. A non-zero energy-momentum tensor will be present in (2.9) when we couple this with matter Lagrangians.) $g_{\mu \nu}$ is taken to be the fundamental field in the theory and represents the spin- 2 graviton. The Lagrangian is quantised in a way analogous to other field theories and so fits neatly with our theories of matter, as hoped. Unfortunately, it can be shown that this theory is nonrenormalisable; that is, it includes divergences which cannot be removed by the addition of a finite number of counterterms (see next chapter).

It is possible to find a Lagrangian which is renormalisable. For instance, Lagrangians of the form $[35,36]$

$$
\dot{\mathcal{L}}=-2 \sqrt{-g}\left(\kappa^{-2} R+\alpha R^{2}+\beta R_{\mu \nu} R^{\mu \nu}\right)
$$

These theories have their own problems. To see this note that the propagator will look like

$$
\frac{1}{p^{2}+\beta \kappa^{2} p^{4}} \sim \frac{1}{p^{2}}-\frac{1}{p^{2}+\kappa^{2} / \beta}
$$

The second term here is a propagator for a massive ghostlike particle, which implies that the theory violates unitarity (see ref. [36] and chapter III of ref. [33]).

The other method which tries to extend (2.10) in a way which deals with the renormalisation problem is supergravity. As is well known, SUSY and SUGRA theories (see refs. $[37,30])$ include symmetries between fermions and bosons. These symmetries would be expected to reduce the divergences in the theory, and maybe remove them completely. 
does not exist any satisfactory quantum theory of gravitation, and in lieu of such a theory it would seem well to gain what experience we can by solving any problems that can be solved with the limited formal apparatus already at our disposal.'

A better understanding of gravity amplitudes and the low energy limit of quantum gravity theories may give pointers to the form of a full consistent theory.

Apart from the direct applications to gravity, this work enables us to explore and develop the scope of the new techniques which we are using. It shows the power of these methods and makes clear the ways they can be generalised to a wider range of situations.

In this work, we will consider calculations for gravity coupled to a range of particles. This includes theories containing spin $0, \frac{1}{2}, 1$ and $\frac{3}{2}$ particles and, in particular, supergravity theories. Before discussing any calculations let us briefly look at the methods for quantising theories involving gravity.

\subsection{Quantisation of Pure Gravity $[28,29,33]$}

Let us begin with the quantisation of pure gravity theory. As we have said, the starting point is the Einstein-Hilbert action

$$
S_{\text {grav }}=-\frac{2}{\kappa^{2}} \int d^{4} x \sqrt{-g} R
$$

Here $g=\operatorname{det}\left(g_{\mu \nu}\right)$ and $R$ is the Ricci scalar, defined above.

In order to quantise the theory we expand the metric as small perturbations around a classical background.

$$
g_{\mu \nu}=\tilde{g}_{\mu \nu}+\kappa h_{\mu \nu}
$$

We will use this to expand all quantities in the theory, the expansion parameter being $\kappa$. $\kappa=\sqrt{8 \pi G}$, where $G$ is Newton's gravitational constant. Note that this implies that $\kappa$ is a dimensional parameter. It is this property which is at the root of the renormalisation difficulties. $h_{\mu \nu}$ will be the quantum of our theory. The indices on $h_{\mu \nu}$ are raised and 
lowered by $\tilde{g}_{\mu \nu}$ and its inverse:

$$
\begin{aligned}
h^{\mu \nu} & =\tilde{g}^{\mu \alpha} h_{\alpha}{ }^{\nu} \\
& =\tilde{g}^{\mu \alpha} \tilde{g}^{\nu \beta} h_{\alpha \beta}
\end{aligned}
$$

The inverse metric is similarly expanded as

$$
g^{\mu \nu}=\tilde{g}^{\mu \nu}-\kappa h^{\mu \nu}+\kappa^{2} h_{\alpha}^{\mu} h^{\alpha \nu}-\kappa^{3} h_{\alpha}^{\mu} h_{\beta}^{\alpha} h^{\beta \nu}+O\left(\kappa^{4}\right)
$$

This enables us to expand the quantities in the Lagrangian as follows: The square root of thè determinant becomes

$$
\sqrt{-g}=\sqrt{-\tilde{g}}\left(1+\frac{\kappa}{2} h+\frac{\kappa^{2}}{8}\left(h^{2}-2 h_{\alpha}^{\beta} h_{\beta}^{\alpha}\right)+\frac{\kappa^{3}}{48}\left(h^{3}-6 h h_{\alpha}^{\beta} h_{\beta}^{\alpha}+8 h_{\alpha}^{\mu} h_{\beta}^{\alpha} h_{\mu}^{\beta}\right)\right)
$$

and inserting the above definition in (I.3) gives the expansion for the Ricci scalar

$$
\begin{array}{r}
R=\tilde{R}+\kappa\left(h_{\beta ; \alpha}^{\beta \alpha}-h_{\beta ; \alpha}^{\alpha \beta}\right) \\
+\kappa^{2}\left(\frac{1}{4} h^{\sigma \lambda ; \mu} h_{\sigma \lambda ; \mu}-\frac{1}{2}\left(h^{\lambda \sigma}\left(2 h_{\sigma \mu}^{; \mu_{\mu}}-h_{\mu_{; \sigma}}^{\mu}\right)\right)_{; \lambda}\right. \\
\quad+\frac{1}{2} h_{\rho ; \beta}^{\tau} h_{\beta ; \tau}^{\rho}-\frac{1}{2} h^{\mu \nu}\left(h_{\beta ; \mu \nu}^{\beta}-2 h_{\mu ; \nu \beta}^{\beta}\right) \\
\left.\quad-\frac{1}{4} h_{\mu ; \tau}^{\mu}\left(2 h_{\alpha}^{\tau ; \alpha}-h_{\nu}^{\nu ; \tau}\right)+h_{\mu}^{\alpha} h^{\mu \beta} \tilde{R}_{\alpha \beta}\right)+O\left(\kappa^{3}\right)
\end{array}
$$

(see appendix I for details of these expansions).

For our purposes, we will always take the classical background to be flat Minkowski space $^{\dagger}$. Hence,

$$
\tilde{g}_{\mu \nu}=\eta_{\mu \nu}
$$

Including this choice of classical background, the action becomes

$$
\begin{aligned}
S_{\text {grav }}=-\frac{2}{\kappa^{2}} \int & d^{4} x\left(\kappa\left(h_{\beta \beta, \alpha \alpha}-h_{\beta \alpha, \beta \alpha}\right)\right. \\
& +\kappa^{2}\left(\frac{1}{2} h\left(h_{\beta \beta, \alpha \alpha}-h_{\beta \alpha, \beta \alpha}\right)+\frac{1}{4} h_{\sigma \lambda, \mu} h_{\sigma \lambda, \mu}+\frac{1}{2} h_{\tau \rho, \beta} h_{\rho \beta, \tau}\right. \\
& \left.\left.-h_{\lambda \sigma, \lambda} h_{\sigma \mu, \mu}+\frac{1}{4} h_{\mu \mu, \tau} h_{\nu \nu, \tau}\right)+O\left(\kappa^{3}\right)\right) .
\end{aligned}
$$

$\dagger$ We choose to define the Minkowski metric, $\eta_{\mu \nu}$, with the signature $(+---)$. 
This will be the implicit starting point in all our calculations. We quantise as usual. For instance, we can define Feynman rules from this Lagrangian, in the same way as we do for other quantum field theories, to allow us to find scattering amplitudes. Note that we have an infinite number of terms in the action; these will lead to interactions with any number of gravitons. It is here that we already begin to see the difficulty of calculations in gravity - with each increase in the number of external particles or number of loops being considered in an amplitude calculation the number of possible interactions in the theory also increases. We can also see hints of the complexity of interaction terms: the low orders shown here already have a large number of terms which must be considered.

As in other field theories, calculations by conventional methods require us to include a gauge fixing term, $-\frac{1}{2} C_{\mu}^{2}$, together with ghost contributions. A usual gauge choice is the de Donder gauge [29], $C_{\mu}=\partial_{\alpha} h_{\mu}^{\alpha}-\frac{1}{2} \partial_{\mu} h$. In fact, none of the new methods here require an explicit choice of gauge or any involvement of ghost contributions. This is one of the many simplifications in the new techniques as compared to the conventional ones.

\subsection{Coupling to Other Particles: Bosons}

To include other particles in our theory we add suitable Lagrangians to the pure gravity . one. Clearly, when adding other particles to our theory we must couple them in some way to our graviton field. In non-supersymmetric quantum gravity, we do this with a 'minimal coupling'. In the case of bosons, this simply means making all derivatives in the added Lagrangian covariant with respect to Lorentz transformations and including the metric explicitly where vertices are contracted. For instance, the Lagrangian for a free massless scalar is

$$
L_{\Phi}=\frac{1}{2} \partial_{\mu} \Phi \partial^{\mu} \Phi
$$

So, for a theory containing scalars coupled to gravity we just add the term

$$
\begin{aligned}
S_{\Phi} & =\frac{1}{2} \int d^{4} x \sqrt{-g} g^{\mu \nu} \nabla_{\mu} \Phi \nabla_{\nu} \Phi \\
& =\frac{1}{2} \int d^{4} x \sqrt{-g} g^{\mu \nu} \partial_{\mu} \Phi \partial_{\nu} \Phi
\end{aligned}
$$


to $S_{\text {grav }}$. Including the expansion (2.14) and condition (2.19), this becomes

$$
\begin{aligned}
S_{\Phi}= & +\frac{1}{2} \int d^{4} x\left(\partial_{\mu} \Phi \partial^{\mu} \Phi+\kappa\left(\frac{1}{2} \eta^{\rho \sigma} h_{\rho \sigma} \eta_{\mu \nu}-h_{\mu \nu}\right) \partial_{\mu} \Phi \partial_{\nu} \Phi\right. \\
& +\kappa^{2}\left(\frac{1}{8}\left(\left(\eta^{\rho \sigma} h_{\rho \sigma}\right)^{2}-2 h_{\alpha \beta} h_{\alpha \beta}\right) \eta_{\mu \nu}-h_{\alpha \mu} h_{\alpha \nu}\right) \partial_{\mu} \Phi \partial_{\nu} \Phi+O\left(\kappa^{3}\right)
\end{aligned}
$$

The graviton and $\Phi$ fields are then quantised.

We will also consider the cases with gravity coupled to massive and complex scalars. As might be expected, the first merely involves adding an extra term to the action

$$
S=S_{\text {grav }}+S_{\Phi}-\int d^{4} x \sqrt{-g} m^{2} \Phi^{2}
$$

For the second the scalar component of the action becomes

$$
S_{\Phi}=+\int d^{4} x \sqrt{-g} g^{\mu \nu} \partial_{\mu} \Phi^{*} \partial_{\nu} \Phi
$$

The practical effect of this will be extra particles in loop calculations and different combinatorics in Feynman rules.

We can include spin 1 particles in an analogous way. The starting point in this case is the Lagrangian

$$
L_{A}=-\frac{1}{4} \operatorname{tr}\left(F_{\mu \nu} F^{\mu \nu}\right)
$$

with

$$
F_{\mu \nu}=\partial_{\mu} A_{\nu}-\partial_{\nu} A_{\mu}+i g\left[A_{\mu}, A_{\nu}\right]
$$

(For photons $\left[A_{\mu}, A_{\nu}\right]=0$.) So, coupling of this field to gravity requires the addition of the term

$$
\begin{aligned}
S_{A}=-\frac{1}{4} \int d^{4} x \sqrt{-g} g^{\alpha \mu} g^{\beta \nu} \operatorname{tr}\left(\left(\nabla_{\mu} A_{\nu}\right.\right. & \left.-\nabla_{\nu} A_{\mu}+i g\left[A_{\mu}, A_{\nu}\right]\right) \\
& \left.\times\left(\nabla_{\alpha} A_{\beta}-\nabla_{\alpha} A_{\beta}+i g\left[A_{\alpha}, A_{\beta}\right]\right)\right)
\end{aligned}
$$

to the pure gravity action and quantisation of the graviton and $A_{\mu}$ fields. We can expand this as above and quantise.

Note again that in both these cases we must consider interactions between two, three or four matter particles and any number of gravitons. Even in the coupling to matter, the calculational problems will increase considerably with higher order calculations. 


\subsection{Coupling to Other Particles: Fermions}

For fermions, the coupling to gravity is more complex [41,42]. Unlike the Lorentz group, it is not possible to construct spinor representations of the local coordinate transformation group $(G L(4, \mathcal{R}))$. The coordinate transformation group is the basic symmetry of GR, so we must find some way to overcome this problem if we wish to include spinors, and hence fermions, in our theory. In order to deal with this we find that we must formulate GR in a slightly different way; in order to obtain a spinor representation we set up a locally inertial space at each point. Since this is Lorentz invariant, we can define representations within it for all quantities, including spinors.

To carry this out in practice, we set up a system of vierbeins, $e_{\alpha}^{a}$, which take us from our original coordinates, $x_{\mu}$, to coordinates, $\xi_{a}$, which are local inertial at each point. In effect, we place all of the coordinate dependence in the vierbeins, leaving us to deal only with the Lorentz aspects of fields. We can then include spinors in the theory with relatively little trouble.

The $e_{\alpha}^{a}$ 's are defined by

$$
e_{\alpha}^{a} \equiv \frac{\partial \xi^{a}}{\partial x^{\alpha}}
$$

and produce a transformation of the metric

$$
e_{\alpha}^{a} e_{\beta}^{b} \eta_{a b}=g_{\alpha \beta}
$$

(Note that the vierbeins can be thought of as the matrix square root of the metric.) We raise Greek indices using the metric tensor and Roman indices using the Minkowski tensor.

The vierbeins can be shown to be both Lorentz and $G L(4, \mathcal{R})$ (coordinate) vectors,

$$
e_{\mu}^{a \prime}(x)=L_{b}^{a}(x) e_{\mu}^{b}(x) \quad e_{\mu}^{a \prime}(\bar{x})=\frac{\partial x^{\nu}}{\partial \bar{x}^{\mu}} e_{\mu}^{b}(x)
$$

and hence we can use them to transform coordinate tensors into Lorentz tensors and coordinate scalars. e.g, a coordinate vector becomes a set of four coordinate scalars which form a Lorentz vector,

$$
A^{a} \equiv e_{\mu}^{a} A^{\mu}
$$


When this is done for all tensors, spinors can be brought into the formulation in a way analogous to any other field - as a set of coordinate scalars which transform as a Lorentz spinor.

As with the metric formulation of GR, we must introduce a covariant derivative. We define this to be

$$
\mathcal{D}_{\mu} \equiv \partial_{\mu}+\frac{1}{2} \sigma^{a b} \omega_{\mu a b}
$$

The $\sigma^{a b}$ are generators of a representation the Lorentz group. The representation depends on the type of quantity being acted on. (The significant case here being spinors, where $\sigma^{a b}=\frac{1}{4}\left[\gamma^{a}, \gamma^{b}\right]$.)

$\omega_{\mu a b}$ is the 'spin connection'; it plays an analogous role to the connection in (2.4). We can define this in a number of ways; the two main conventions are the 'first' and 'second' order formalisms. These give two different 'minimal couplings' of fermions to gravity. In the first order formalism we begin by taking $\omega_{\mu a b}$ to be an independent field and subsequently eliminate it by solving its field equation, $\delta S / \delta \omega_{\mu a b}=0$. We will use this method in the next section to construct the simplest form of $N=1$ SUGRA. Here, we outline the second order formalism. This is the usual choice for fermions in non-SUGRA theories. The definition follows the method for defining the metric connection in the metric formulation of GR. In that case we require that the covariant derivative of the metric tensor vanishes. Here, we define the the second order spin connection, $\omega_{\mu a b}(e)$, by

$$
\partial_{\mu} e_{\nu}^{a}+\omega_{\mu}^{a b}(e) e_{b \nu}-\Gamma_{\nu \mu}^{\rho} e_{\rho}^{a}=0
$$

Which implies that

$$
\omega_{\mu a b}=e_{a}^{\nu}\left(\partial_{\mu} e_{b \nu}-\partial_{\nu} e_{b \mu}+\frac{1}{2} e_{a}^{\nu} e_{b}^{\rho}\left(\partial_{\rho} e_{c \nu}-\partial_{\nu} e_{c \rho} e_{\mu}^{c}\right)\right)+(a \leftrightarrow b)
$$

(This is, in fact, the solution of $\delta S / \delta \omega_{\mu a b}=0$ when $S$ is the pure gravity action.) 
In order for us to use the correct measure in the action integration, we must also define $\sqrt{-g}$ in terms of the vierbeins. We find, in agreement with the suggestion that the vierbeins are the 'matrix square root' of the metric, that it is simply

$$
\sqrt{-g}=e \equiv \operatorname{det}\left(e_{\alpha}^{a}\right)
$$

In terms of the vierbein formalism the pure gravity Lagrangian becomes

$$
L_{\text {grav }}=-\frac{1}{2 \kappa^{2}} e R=-\frac{1}{2 \kappa^{2}} e R_{\mu \nu}^{a b} e_{a}^{\nu} e_{b}^{\mu}
$$

Once we have written quantities in this vierbein formalism of GR, we can easily find the minimally coupled Lagrangian as before - we simply change derivatives to covariant derivatives wherever they occur. However, before we discuss the details of this, we must look at how we handle the gravitons in this formalism. The gravity field is now represented by the vierbeins, so we would expect that these should be expanded in some way. Obviously, for the simplest application of the theory we would like to expand the vierbeins in a way which meshes with our formulation for bosons. If this were not possible we would have to rewrite the bosonic terms (and, in particular, the pure gravity terms) using vierbeins whenever we included fermions in our theory. While this is possible it is not ideal by any means. Fortunately, it has been shown that, for suitable gauge choices, we can write the vierbeins so that the metric is the field variable. i.e., we can expand $e_{\mu}^{a}$ in terms of $h_{\mu \nu}$ and use both GR formulations in parallel [43]. The expansion of the vierbein is

$$
e_{\mu}^{a}=\tilde{e}_{\mu}^{a}+\frac{\kappa}{2} h_{\mu \beta} \tilde{g}^{-1 \beta \nu} \tilde{e}_{\nu a}-\frac{\kappa^{2}}{8}\left(h_{\mu \beta} \tilde{g}^{-1 \beta \nu} h_{\nu \rho} \tilde{g}^{-1 \rho \alpha} \tilde{e}_{\alpha a}\right)+O\left(\kappa^{3}\right)
$$

where $\tilde{g}^{\beta \nu}$ and $\tilde{e}_{\alpha a}$ are respectively the metric and vierbein fields representing the classical background.

With our choice of $\tilde{g}^{\beta \nu}=\eta^{\beta \nu}$ this expansion is

$$
e_{\mu}^{a}=\delta_{\mu}^{a}+\frac{\kappa}{2} h_{\mu}^{a}-\frac{\kappa^{2}}{8} h_{\mu \alpha} h^{\alpha a}+O\left(\kappa^{3}\right)
$$

(It is trivial to check that (2.39) and (2.30) imply (2.14).) 
Having constructed a way of introducing fermions into GR and found the above expansion to relate this to our other results, we are now in a position to define our theory with gravity-fermion couplings in a way which fits with the boson couplings of the last section. We begin with spin- $\frac{1}{2}$ fermions.

As is well known, the Lagrangian for a free massless spin- $\frac{1}{2}$ fermion is

$$
L_{\eta}=\frac{i}{2}\left(\bar{\eta} \gamma^{\mu}\left(\partial_{\mu} \eta\right)-\left(\partial_{\mu} \bar{\eta}\right) \gamma^{\mu} \eta\right)
$$

We must convert the derivatives, $\partial_{\mu}$, to covariant derivatives and insert vierbeins where appropriate. So, the extra term to be added to the action is

$$
S_{\eta}=\frac{i}{2} \int d^{4} x e\left(\bar{\eta} \gamma^{a} e_{a}^{\mu}\left(\mathcal{D}_{\mu} \eta\right)-\left(e_{a}^{\mu} \mathcal{D}_{\mu} \bar{\eta}\right) \gamma^{a} \eta\right)
$$

$\gamma^{a}$ are the usual constant $\gamma$-matrices. We can use (2.39) to give us an action which can be quantised as before.

Similarly, we can look at spin-3/2 fermions. The kinetic-energy term which we must use for this field is the Rarita-Schwinger term [44]:

$$
L_{\Psi}=-\frac{1}{2} \epsilon^{\mu \nu \rho \sigma} \bar{\Psi}_{\mu} \gamma_{5} \gamma_{\nu} \partial_{\rho} \Psi_{\sigma}
$$

Again, to couple this to gravity, we must convert the derivative to a covariant one. For simplicity of description we initially mix the two GR formulations in our description of this field $[45,46]$; we consider the field to have a coordinate vector index and a Lorentz spinor index. i.e., $\Psi=\Psi_{\mu \alpha}$.

Then, the action of the covariant derivative on this term must take the form

$$
\mathcal{D}_{\nu} \Psi_{\mu \alpha}=\partial_{\nu} \Psi_{\mu \alpha}-\Gamma_{\mu \nu}^{\lambda} \Psi_{\lambda \alpha}+\frac{1}{2} \omega_{\nu a b}\left(\sigma^{a b}\right)_{\alpha}^{\beta} \Psi_{\mu \beta}
$$

where $\omega_{\mu a b}$ and $\sigma_{a b}$ are those defined above. Note that in the Lagrangian the symmetric indices on $\Gamma$ are contracted with the Levi-Civita tensor and so the middle term on the right-hand side vanishes in the Lagrangian - we may regard the derivative to be with 
respect to the spin- $\frac{1}{2}$ content of $\Psi$ only. Hence, we can ignore the $\Gamma \Psi$ term and need not worry about the mixing of the GR formulations.

Assuming this definition for $\mathcal{D}$, we can write the complete action for the gravitygravitino system as:

$$
S_{\text {grav } / \Psi}=\int d^{4} x\left(-\frac{2 \sqrt{-g}}{\kappa^{2}} R-\frac{1}{2} \epsilon^{\mu \nu \rho \sigma} \bar{\Psi}^{\mu} \gamma_{5} \gamma^{a} e_{a}^{\rho} \mathcal{D}_{\rho} \Psi_{\sigma}\right)
$$

This can then be expanded and quantised.

\subsection{Supergravity $[30,46]$}

As we have already mentioned, supersymmetric theories are theories which contain symmetries between bosons and fermions. These symmetries imply that there will be cancellations in amplitude calculations and simplifications in final expressions. The hope that cancellations will remove problematic divergences has generated a great deal of interest in SUSY theories which contain gravity.

While a spin-2 (graviton) field can be included in a global SUSY theory (see, e.g., ref. [46]), the typical way of creating a theory with gravity and SUSY is to use local supersymmetry (see ref. [30]). In fact, it is easy to show that a gauged supersymmetric theory requires the introduction of a spin- 2 boson and its spin- $\frac{3}{2}$ partner. We can identify the spin-2 particle with the graviton and so find that theories with local supersymmetry must contain gravity. We name this class of theories 'supergravity' (SUGRA).

The simplest SUGRA theory is one containing a 'single supersymmetry' and a single doublet containing a graviton and a gravitino (the spin- $\frac{3}{2}$ particle). Evidently, the starting point for such a theory will be the Lagrangian found in the final last section for a theory coupling these two particles (2.44). There are then two ways to approach the problem of 'supersymmetrising' this. The first historically was to add interaction terms which make the Lagrangian invariant under SUSY transformations [45]:

$$
L_{i n t}=\frac{1}{32}\left(\bar{\Psi}^{a} \gamma^{b} \Psi^{c}\left(\bar{\Psi}_{a} \gamma_{b} \Psi_{c}+2 \bar{\Psi}^{b} \gamma^{a} \Psi^{c}\right)-4\left(\bar{\Psi}^{a} \gamma \cdot \Psi\right)^{2}\right)
$$


The Lagrangian is then invariant under the local SUSY transformation:

$$
\begin{aligned}
& \delta e_{\mu}^{a}=\frac{\kappa}{2} \bar{\epsilon} \gamma^{a} \Psi_{\mu} \\
& \delta \Psi_{\mu}=\frac{1}{\kappa} \mathcal{D}_{\mu} \epsilon
\end{aligned}
$$

"It is, however, possible to construct a simpler form of the action if one takes $\omega_{\mu a b}$ to be an independent field from the beginning [47]. Then we find that our supersymmetric Lagrangian is just

$$
L_{N=1}=-\frac{1}{2 \kappa^{2}} e R-\frac{1}{2} \epsilon^{\mu \nu \rho \sigma} \bar{\Psi}^{\mu} \gamma_{5} \gamma^{a} e_{a}^{\rho} \mathcal{D}_{\rho} \Psi_{\sigma}
$$

This is invariant under the transformations

$$
\begin{aligned}
& \delta e_{\mu}^{a}=\frac{\kappa}{2} \bar{\epsilon} \gamma^{a} \Psi_{\mu} \\
& \delta \Psi_{\mu}=\frac{1}{\kappa} \mathcal{D}_{\mu} \epsilon \\
& \delta \omega_{\mu a b}=\bar{\epsilon} \gamma_{5} \gamma_{\mu} \mathcal{D}_{\rho} \Psi_{\nu} \epsilon^{a b \nu \rho}-\frac{1}{2} e_{\mu}^{b} \bar{\epsilon}_{5} \gamma_{\sigma} \mathcal{D}_{\rho} \Psi_{\nu} \epsilon^{a \sigma \nu \rho} \\
& +\frac{1}{2} e_{\mu}^{a} \bar{\epsilon} \gamma_{5} \gamma_{\sigma} \mathcal{D}_{\rho} \Psi_{\nu} \epsilon^{b \sigma \nu \rho}
\end{aligned}
$$

This is the so-called second order formalism for the spin connection. If we solve the field equation

$$
\frac{\delta S}{\delta \omega_{\mu a b}}=0
$$

we find that $\omega_{\mu a b}$ is given by

$$
\omega_{\mu a b}=\omega_{\mu a b}(e)+\kappa_{\mu a b}
$$

Here, $\omega_{\mu a b}(e)$ is the connection defined in equation (2.34). $\kappa_{\mu a b}$ is defined as

$$
\kappa_{\mu a b}=\frac{\kappa^{2}}{4}\left(\bar{\Psi}_{\mu} \gamma_{a} \Psi_{b}-\bar{\Psi}_{\mu} \gamma_{b} \Psi_{a}+\bar{\Psi}_{a} \gamma_{\mu} \Psi_{b}\right)
$$

This extra term can be interpreted as implying that gravitinos add torsion to our theory. Note that the two actions are equivalent on-shell. Since our calculations are restricted to on-shell amplitudes, we will not need to distinguish between the different formulations in the results discussed here. Thus, we have formulated a theory with a single local SUSY invariance. 
We can also regard this from an operator perspective. In this view, we have an operator, $Q$, which transforms between the creation and annihilation operators of the theory. So, in the theory above we would have a $Q$ such that

$$
\begin{aligned}
{\left[Q, a_{g}^{ \pm}\right] } & = \pm \alpha_{1} a_{\psi}^{ \pm} \\
\left\{Q, a_{\psi}^{ \pm}\right\} & = \pm \alpha_{2} a_{g}^{ \pm}
\end{aligned}
$$

where $a_{g}^{ \pm}$and $a_{\psi}^{ \pm}$are creation and annihilation operators for gravitons and gravitinos (of helicity \pm 1 ) respectively. Since we have only one operator this is designated to be a $N=1$ SUGRA theory. In this formulation we implicitly have two SUSY doublets, one containing the positive helicity particles and the other containing the negative helicity ones. We can easily extend the theory by coupling other $N=1$ SUSY doublets [48]. These need not necessarily be $\left(2, \frac{3}{2}\right)$ doublets.

It is possible to create theories containing more than one SUSY operator - that is, with more that one supersymmetry between particles. Starting with our $N=1$ Lagrangian, it can be shown that, if we couple a $\left(\frac{3}{2}, 1\right)$ doublet, it is possible to extend the Lagrangian in such a way that the two spin- $\frac{3}{2}$ fields can be rotated into each other. This implies that the theory has a second SUSY transformation similar to the original one but with the spin $-\frac{3}{2}$ fields swapping roles. From an operator point of view we now have two SUSY operators; the theory is $N=2$ SUGRA [49]. The four particles comprise a complete $N=2$ multiplet. (In general, we will include two multiplets containing sets of positive and negative helicity particles.)

In this way, we can continue to add more particles and increase the number of operators [50,51]. The Lagrangians become increasingly complicated, so we will not give any of them in detail here. A theory can contain any number of multiplets with different combinations of particles related by the supersymmetry. So, for instance, in an $N=2$ theory we may have the 2 multiplets described above plus a matter multiplet containing two scalars and a spin- $\frac{1}{2}$ fermion. The natural limit of increasing SUSY operators seems to be $N=8$ SUGRA. Beyond this point we must add a spin- $\frac{5}{2}$ particle into the theory. 
There are difficulties in coupling such a field with other particles and, anyway, there is no reason to believe that nature contains anything beyond spin-2 [30,52].

The $N=8$ SUGRA [53] has one possible multiplet containing the particle content: 1 graviton, 8 gravitinos, 28 vectors, 56 spin- $\frac{1}{2}$ fermions and 35 (complex) scalars. This SUGRA has been of great interest to theorists. Since it contains a very high degree of symmetry it could be that this overcomes completely the problems of infinities found in quantum gravity. Also, we might hope that the theory has a symmetry group which could be broken to give the standard model and all its particles. Unfortunately, the second of these seems to be untrue in practice [46]; the first we will discuss later.

\subsection{Previous Calculations in Perturbative Quantum Gravity}

There are two sets of calculations which are relevant in quantum gravity, perturbative amplitudes and counterterms. Obviously, these are, strongly intertwined, the first often giving information for the second. We will leave the problem of counterterms to the next section, where we discuss the divergences and renormalisation in PQG. Here we look at explicit calculations of amplitudes.

Due to the difficulties involved in calculating quantities in quantum field theories, we often consider a perturbative expansion. That is, we take a small parameter (usually, a coupling constant) and limit our calculations to specific orders in that parameter. This is the most widespread way for finding scattering amplitudes for particle interactions. The majority of these calculations are carried out using the Feynman diagram technique (though we will also mention cases where string theory has been used in the past). Since this technique is widely used in particle physics we will only give a brief review of the essential aspects of the method.

The Feynman rules are constructed to provide a straightforward, graphical and compact way of calculating Green functions or scattering amplitudes in quantum field theories. The rules are constructed from the Lagrangian of the theory being considered (including 
a suitable gauge fixing condition and ghost contribution). They consist of a set of 'vertices' and 'propagators'; the former describing the point interactions of the particles in the theory and the latter their movement between these interactions. These components are used to construct diagrams representing perturbative processes in the theory. Each vertex and propagator is associated with an algebraic formula; these are used, together with the diagrams, to construct integral expressions for the Green functions of the theory.

Since each vertex carries a power of one of the coupling constants of the theory we can easily separate Feynman diagram results into contributions at the same perturbative order. For a fixed number of external particles the diagrams can be classed according to the number of loops which they contain; the higher the number of loops, the higher the perturbative order a diagram will contribute to. So, the lowest order is found be summing all tree diagrams - that is, those with no internal loops; the next will be the sum of all diagrams with one loop; and so on.

In PQG we can carry out this process in precisely the same way as other field theories; the rules are derived from the Lagrangian and calculations at a particular perturbative order can be carried out. (For an example of a complete set of Feynman rules see ref. [54].) . We have already hinted at the significant difference between this and most other field theories - the expansion of the Lagrangian contains an infinite number of terms, so we will have an infinite number of vertices which must be considered. Each vertex is also typically more complex than analogous ones in other theories and integrals will contain more powers of momenta, in general. These all work to increase the difficulty of calculations in PQG.

There is one further step which is often made in gravity formulations: the background field method [55]. This simplifies calculations and retains some of the symmetry of the theory. It is primarily used to produce an effective action with has the same symmetries as the theory. It is particularly useful when considering counterterms, since it allows us to write them in a form in which all symmetries are seen explicitly [56].

This method is formulated within an effective action description of the theory and 
so is relevant, in the first instance, for 1 particle irreducible diagrams. The basic step in the background field method is to make a split of the fields into background and quantum parts. So in gravity we will have an additional split to the one in (2.14):

$$
h_{\mu \nu}=\hat{g}_{\mu \nu}+\phi_{\mu \nu}
$$

( $\hat{g}_{\mu \nu}$ is the 'background' field and $\phi_{\mu \nu}$ the 'quantum' one). (We will use " $"$ to indicate quantities which depend only on background quantities.) The effective action is considered to be an integral only over the quantum fields; only these can appear in loops. Conversely, only background fields have source terms; only these can appear as external lines in diagrams. So, our 1PI diagrams consist of external $\bar{g}_{\mu \nu}$ particles with loops of $\phi_{\mu \nu}$ particles.

We find that the theory now has two gauge symmetries: one in which the background fields do not transform and the other in which all fields transform, the background ones with the original gauge symmetry of the theory. So, we can just break the first symmetry and leave all the results as functions of the background fields which retain the symmetries of the theory. This way we will recover an effective action which obeys all the theory's symmetries.

We can use this for general amplitude calculations, as was shown in ref. [57]. Breaking the second gauge symmetry allows us to introduce a propagator for the background field with which we can join the 1 PI diagrams. So, we will have loops containing quantum fields joined by background field propagators. As we have broken the quantum field and background field symmetries independently, there is no reason that they should have the same gauge choice. So, surprisingly, we find that we have a method in which we can use different gauges in the loop and tree parts of our calculations.

It is in this background gauge method form that we will implicitly consider the counterLagrangian. In this way the counterterms retain the symmetries seen in the underlying theory. It also seems that this is the gauge implied by the string rules; to find a field theory mapping for these rules we are required to formulate our theory in precisely this way (see 
ref. [58]). The field theory mapping for the QCD rules implies that the most efficient method of calculation in that situation is to use the background field method plus the Gervais-Neveu [59] gauge on the trees.' In the string-inspired rules for gravity, we require the background gauge plus the de Donder gauge and a slight redefinition of the fields [31].

Now let us catalogue the previous calculations in PQG. As with all field theories, the calculations of gravity tree amplitudes are reasonably straightforward using Feynman diagrams. There is no integration required, so the results come almost immediately after the rules have been derived. There are two cases which are of interest here because of their use of string theory. In ref. .60], Sannan obtained the tree-level 4-graviton scattering amplitude both directly and by looking at the infinite tension limit of a closed string theory. The latter is close in spirit to the work which we will present here. Berends, Giele and Kuijf [20] combined this with the fact that open and closed string tree scattering amplitudes could be related [61]. This allowed them to relate gluon and graviton amplitudes and find new graviton results at tree level. By this method, they obtained a conjecture for $n$-graviton amplitudes of the form $A^{\text {tree }}\left(1^{-}, 2^{-}, 3^{+}, \ldots, n^{+}\right)$and confirmed it numerically for $n \leq 11$.

The majority of one-loop calculations using Feynman diagrams in perturbative gravity have been corrections to the propagator. These have been calculated for all particles types in refs. $[62,63]$. Corrections to the propagator are the simplest calculations at any order, so this is perhaps the obvious starting point. The results found gave some indication of the divergences in PQG.

Probably the most heroic attempt at using the traditional methods for one-loop gravity calculations was by Grisaru and Zak in ref. [64]. They calculated all one-loop four graviton amplitudes with internal massless scalars. We reproduce these results, using the stringinspired and Cutkosky rules, as part of a more general set of one-loop results.

The calculation which is closest in spirit to the work done here is that by Green, Schwarz and Brink in ref. [65]. They obtained the four-graviton $N=8$ supersymmetric 
amplitude by looking at the field theory limit of a closed string theory. Again, we reproduce this result with both the string-inspired and Cutkosky rules.

The other Feynman diagram calculation at one-loop was carried out by Deser and van Nieuwenhuizen [42]. They looked at the scattering of eight fermions. Their purpose was to identify non-zero counterterms in the Dirac-Einstein system and so they concentrated only on the divergent contribution of the diagram. We will look at this in the next section where we consider the wider issue of divergences and counterterm determination in PQG. 
I can't help it, the idea of the infinite torments me.

Alfred de Musset

\section{Divergences and Renormalisation .}

Any discussion of gravity in quantum field theory must include some consideration of renormalisability, since this is one of the major problems for anyone wishing to construct a quantum gravity theory. Our work here will be no exception: many of the results will relate directly to the problem and we must keep it in mind even when they do not. For this reason, we will devote this chapter to an outline the issue of renormalisation and divergences in PQG and SUGRA.

\subsection{Renormalisation [66]}

In general, if we write down some Lagrangian for a quantum field theory we will find that infinities appear in calculations we carry out. These infinities come in two forms. - infrared and ultraviolet (coming from the low and high momentum sector of integrals, respectively). We will discuss the infrared divergences in chapter 7; they do not concern us too much since we know that they will eventually vanish in the S-matrix $[1,67]$. If the only divergences in a theory are IR then the theory is finite and we have no problems. If, on the other hand, the theory contains ultraviolet divergences, we will have problems if we cannot deal with them in a sensible way.

The usual method for removing any UV divergences which occur in a particular theory is to add 'counterterms' to the Lagrangian. These are extra terms which act to remove the infinities from the results. In perturbative calculations it may be that counterterms must be added at each order. This process of adding counterterms is fine if the new terms are the same as the terms already in the Lagrangian or if we only need to add a finite number. 
The first case merely implies that the 'physical' parameters which we chose were wrong; the second that we chose the wrong Lagrangian. In both these cases we call the theory renormalisable.

The problem comes if an infinite number of counterterms is required to make a theory finite. This will happen if, for instance, we must add a new type of term at each perturbative order. In this case we lose predictability (since, in effect, we must rewrite the theory at each order) and the theory is non-renormalisable.

Any theory containing perturbative gravity will theoretically be non-renormalisable, a fact which can be attributed to its dimensional coupling constant: At each order we must add an additional momentum in the numerator to balance the extra power of the coupling constant. Hence, as the number of loops being considered gets larger there is potential for increasingly divergent contributions to the amplitude. As divergences at each order will be of different type, their presence will require an infinite set of counterterms.

Of course, this non-renormalisability would not be a problem if these potential divergences vanish order-by-order in the theory and the perturbative expansion was finite. In this case all potential counterterms will have zero coefficients and be irrelevant. Proof or disproof of this has been a significant area of research in gravity, especially for the case of SUGRA theories. Here we give a brief review of the results obtained. We begin with general 1-loop results, followed by two-loop pure gravity and finish with a discussion of the status in SUGRA.

Before we start, note that in all calculations and discussions of divergences we will implicitly assume that regularisation is carried out by use of a dimensional regularisation [68] or dimensional reduction [69] scheme. In both these schemes we analytically continue the dimension we are working in away from 4 to $4-2 \epsilon$. In the former we decrease the number of states accordingly; in the latter we assume the change in dimensions is due to compactification, so that the number of states is constant. Dimensional reduction is important in the supersymmetric theories, since it retains supersymmetric cancellations. 
It can be shown that the two are equivalent (up to redefinition of the coupling) [69]. In both schemes divergences are seen as poles, $1 / \epsilon^{n}$. Note further, that when calculating in $4-2 \epsilon$ dimensions, the dimension of $\kappa$ will change. To compensate for this we should make the redefinition $\kappa \rightarrow \mu^{\epsilon} \kappa$, where $\mu$ is some arbitrary mass scale [63]; $\kappa$ can then retain its four-dimensional value throughout. However, for simplicity, we will not make this change explicitly, but will assume that it can be done where necessary.

\subsection{One-Loop Divergences}

When it was realised that gravity was potentially a non-renormalisable theory, it was hoped that there might be some 'hidden symmetries' in the theory which would force it to be finite at all orders. To make some progress towards discovering whether this was true, a number of calculations of one loop results were carried out. The first of these was by 't Hooft and Veltman. They were able to show via simple arguments that pure gravity is in fact finite on-shell at one-loop [56]. Unfortunately, this success did not carry over to other situations. A simply algorithm showed [70] that pure gravity off-shell and gravity coupled to scalar particles are both divergent [56]. Deser and van Nieuwenhuizen [71] used this algorithm to show the same for gravity coupled to a photon and (together with Tsao) for gravity coupled to Yang-Mills particles [72]. They also found, this time via direct calculation, that the Einstein-Dirac system is no better [42].

We will describe these calculations below, but first let us begin with the proof of a useful theorem: When dealing with external on-shell particles, we can use the classical field equations to simplify the counter-Lagrangian. This was shown by 't Hooft [70]: Consider a general Lagrangian, dependent on fields $\phi_{i}$,

$$
\mathcal{L}\left(\phi_{i}, \lambda\right)=\mathcal{L}_{0}\left(\phi_{i}\right)+\Delta \mathcal{L}\left(\phi_{i}, \lambda\right)
$$

$\Delta \mathcal{L}$ is the counter-Lagrangian, $\Delta \mathcal{L} \sim 1 / \epsilon$, and is dependent on a parameter $\lambda, \Delta \mathcal{L} \sim \lambda$. We will consistently discard terms $\sim \mathcal{O}\left(\lambda^{2}\right)$. A field redefinition should not affect any 
results, so let us look at the effect of changing $\phi_{i}$ by

$$
\phi_{i} \rightarrow \phi_{i}+\delta \phi_{i}
$$

with $\delta \phi_{i} \sim \lambda$. Inserting this into the Lagrangian gives

$$
\mathcal{L}_{0}\left(\phi_{i}+\delta \phi_{i}\right) \rightarrow \mathcal{L}_{0}\left(\phi_{i}\right)+\mathcal{L}_{0}^{\prime}\left(\phi_{i}\right) \delta \phi_{i}+\mathcal{O}\left(\lambda^{2}\right)
$$

$\delta \phi_{i}$ can contain poles of $\epsilon$ and, if so, will require a change in the counter-Lagrangian of $-\mathcal{L}_{0}^{\prime}\left(\phi_{i}\right) \delta \phi_{i} . \mathcal{L}_{0}^{\prime}=0$ are the classical field equations, so this suggests that we can use these to simplify the counter-Lagrangian.

\section{On-shell Pure Gravity}

It is, in fact, quite straightforward to show the on-shell finiteness of pure gravity by simple arguments concerning the counterterms:

To find all possible one-loop counterterms consider first that the degree of divergence of a one-loop calculation will be $\mathcal{O}\left(k^{4}\right)$. We must therefore look for counterterms containing four derivatives. We can restrict our search to terms which are invariant functions of the background field, since the counter-Lagrangian should have the same symmetries as the bare one.

A moment's thought shows that this implies we must build terms containing just two Riemann tensors or one Riemann tensor and two covariant derivatives. Further, it is easy to see that any term with derivatives must be exact derivative, and so will not affect the action. We are left with three candidate counterterms:

$$
\begin{gathered}
\hat{R}^{2} \\
\hat{R}_{\mu \nu} \hat{R}^{\mu \nu} \\
\hat{R}_{\mu \nu \sigma \rho} \hat{R}^{\mu \nu \sigma \rho}
\end{gathered}
$$

(In all cases these terms depend only on the background fields.) We can simplify this further by implementing the well-known Gauss-Bonnet formula

$$
\int d^{4} x \sqrt{-g}\left(\hat{R}_{\mu \nu \sigma \rho} \hat{R}^{\mu \nu \sigma \rho}-4 \hat{R}_{\mu \nu} \hat{R}^{\mu \nu}+\hat{R}^{2}\right)=0
$$


This allows us to remove one of the three terms in the action in favour of the other two. Thus, we find our counter-Lagrangian must take the form

$$
\Delta L \sim \sqrt{-g}\left(\alpha \hat{R}_{\mu \nu} \hat{R}^{\mu \nu}+\beta \hat{R}^{2}\right)
$$

In general, when we are considering external on-shell particles, we can use the classical field equations of the theory to simplify the counter Lagrangian. In this case the field equations are simply $\hat{R}_{\mu \nu}(\hat{g})=0$. Clearly, inserting these into (3.6) will give the result $\Delta L=0$ implying that any pure gravity amplitudes with external on-shell gravitons are finite.

\section{Off-shell Pure Gravity}

If we wish to consider the divergences for amplitudes which are not on-shell we must find the values of $\alpha$ and $\beta$. In general this involves a complicated calculation, but the 't HooftVeltman algorithm reduces the work considerably by recasting the theory in the form of a Lagrangian of scalar fields. With only the calculation of simple vacuum energy diagrams, they showed that a Lagrangian of the form

$$
\mathcal{L}=\sqrt{-g}\left(\partial_{\mu} \phi_{i}^{*} g^{\mu \nu} \partial_{\nu} \phi_{i}-2 \phi_{i}^{*} \mathcal{N}^{\mu} \partial_{\mu} \phi_{i}+\phi_{i}^{*} \mathcal{M} \phi_{i}\right)
$$

(where $\mathcal{N}^{\mu}$ and $\mathcal{M}$ are functions of external fields) will have the counter-Lagrangian

$$
\begin{array}{r}
\Delta \mathcal{L}=\frac{\sqrt{-g}}{(4 \pi)^{2} \epsilon}\left(\frac{1}{12} \mathcal{Y}^{\mu \nu} \mathcal{Y}_{\mu \nu}+\frac{1}{2}\left(\mathcal{M}+\mathcal{N}^{\mu} \mathcal{N}_{\mu}+D_{\mu} \mathcal{N}^{\mu}-\frac{1}{6} R\right)^{2}\right. \\
\left.+\frac{1}{60}\left(R_{\mu \nu} R^{\mu \nu}-\frac{1}{3} R^{2}\right)\right)
\end{array}
$$

with $\mathcal{Y}_{\mu \nu}=D_{\mu} \mathcal{N}_{\nu}-D_{\nu} \mathcal{N}_{\mu}+\mathcal{N}_{\mu} \mathcal{N}_{\nu}-\mathcal{N}_{\nu} \mathcal{N}_{\mu}$

In ref. [56], this was applied to pure gravity by carrying out a background field expansion of the gravity field, arranging the Lagrangian in a suitable form and identifying the ten independent fields in $h_{i j}$ (recall that $h_{i j}$ is symmetric) with $\phi_{1}, \ldots, \phi_{10}$.

This gives the counter-Lagrangian for off-shell gravity to be

$$
\Delta L_{g r a v}=-\frac{\sqrt{-g}}{(4 \pi)^{2} \epsilon}\left(\frac{1}{120} \hat{R}^{2}+\frac{7}{20} \hat{R}_{\mu \nu} \hat{R}^{\mu \nu}\right)
$$

(Note that this is of the form (3.6), in agreement with the general arguments given above.) 


\section{Gravity coupled to Bosons}

't Hooft and Veltman also applied the same algorithm to a theory with one scalar coupled to gravity.

$$
L=-\frac{2 \sqrt{-g}}{\kappa^{2}} R+\frac{\sqrt{-g}}{2} \partial_{\mu} \phi g^{\mu \nu} \partial_{\nu} \phi
$$

To do this, they simply extended the set of fields $\phi_{i}$ by identifying the extra scalar with $\phi_{11}$. In this way, they found the counter-Lagrangian

$$
\begin{aligned}
\Delta L=-\frac{\sqrt{-g}}{(4 \pi)^{2} \epsilon}\left(\frac{9}{720} \hat{R}^{2}+\frac{43}{120} \hat{R}_{\mu \nu} \hat{R}^{\mu \nu}\right. & +\frac{\kappa^{4}}{2}\left(\partial_{\mu} \hat{\phi} g^{\mu \nu} \partial_{\nu} \hat{\phi}\right)^{2} \\
& \left.+\frac{\kappa^{2}}{12} \hat{R}\left(\partial_{\mu} \hat{\phi} g^{\mu \nu} \partial_{\nu} \hat{\phi}\right)+2 \kappa^{2}\left(D_{\mu} D^{\mu} \hat{\phi}\right)^{2}\right)
\end{aligned}
$$

( $\hat{\phi}$ is the background scalar field). Note that in this case there are divergences for on-shell as well as off-shell particles; unlike the previous case, the classical field equations do not remove all the terms in the counter-Lagrangian. We can, however, use the field equations to simplify (3.11) when the external particles are on-shell. The relevant equations in this case are

$$
\begin{aligned}
D_{\mu} D^{\mu} \hat{\phi} & =0 \\
\hat{R}_{\mu \nu} & =\frac{\kappa^{2}}{2}\left(D_{\mu} \hat{\phi}\right)\left(D_{\nu} \hat{\phi}\right) \\
\hat{R} & =\frac{\kappa^{2}}{2}\left(D_{\mu} \hat{\phi}\right)\left(D^{\mu} \hat{\phi}\right)
\end{aligned}
$$

Implying the counter-Lagrangian can simplified as

$$
\Delta L=-\frac{\sqrt{-g}}{(4 \pi)^{2} \epsilon} \frac{203}{80} \hat{R}^{2}=-\frac{\sqrt{-g}}{(4 \pi)^{2} \epsilon} \frac{203}{320} \kappa^{4}\left(D_{\mu} \hat{\phi} D^{\mu} \hat{\phi}\right)^{2} .
$$

The 't Hooft-Veltman algorithm was also used to show that gravity coupled to elecromagnetism and gravity coupled to Yang-Mills fields both contain divergences $[71,72]$. The counter-terms for an Einstein-Maxwell Lagrangian are

$$
\begin{aligned}
\Delta L=-\frac{\sqrt{-g}}{(4 \pi)^{2} \epsilon}\left(\frac{9}{20} \hat{R}_{\mu \nu} \hat{R}^{\mu \nu}-\frac{1}{40} \hat{R}^{2}\right. & +\frac{\kappa^{2}}{3} \hat{R}_{\mu \nu} \hat{T}^{\mu \nu} \\
& \left.+\frac{13}{6} \kappa^{4} \hat{T}_{\mu \nu} \hat{T}^{\mu \nu}-\frac{\kappa^{2}}{3} \operatorname{tr}\left(D^{\alpha} \hat{F}_{\alpha \nu} D^{\beta} \hat{F}_{\beta}^{\nu}\right)\right)
\end{aligned}
$$


where, as usual, $\hat{F}_{\mu \nu}=\partial_{\mu} \hat{A}_{\nu}-\partial_{\nu} \hat{A}_{\mu}$ and $\hat{T}_{\mu \nu}{ }^{\prime}=\hat{F}_{\mu \alpha} \hat{F}_{\nu}^{\alpha}-\frac{1}{4} g_{\mu \nu} \hat{F}^{\alpha \beta} \hat{F}_{\alpha \beta}\left(\hat{A}_{\mu}\right.$ is the background photon field). Using the field equations, we can simplify this to

$$
\Delta L=-\frac{\sqrt{-g}}{(4 \pi)^{2} \epsilon} \frac{137}{60} \hat{R}_{\mu \nu} \hat{R}^{\mu \nu}
$$

when the external particles are on-shell. This can be generalised to the (on-shell) YangMills case as

$$
\Delta L=-\frac{\sqrt{-g}}{(4 \pi)^{2} \epsilon}\left(\left(\frac{137}{60}+\frac{r-1}{10}\right) \hat{R}_{\mu \nu} \hat{R}^{\mu \nu}-\frac{11}{24} C\left(\frac{f}{\kappa}\right)^{2}\left(\hat{F}^{a} \hat{F}^{a}\right)\right)
$$

where $\mathrm{r}$ is the dimension of the gauge group, $f$ is the Yang-Mills coupling constant and $r C=C^{a b c} C_{a b c}$, the product of the structure functions.

\section{Gravity coupled to Fermions}

The 't Hooft-Veltman algorithm is not applicable to the calculation for the Dirac-Einstein Lagrangian without significant adaption; it cannot be used in the form above when fermions are involved. Deser and van Nieuwenhuizen [42] were able to show that one of the counterterm coefficients can be calculated by looking at one set of diagrams - those with eight external fermions and internal gravitons. (In fact, only the divergent parts of this diagram are needed to extract the relevant information.) The coefficients found in this calculation are sufficient to prove that this system will also contain divergences.

In general, we would expect the counter-Lagrangian to take the form

$$
\begin{array}{r}
\Delta \mathcal{L}=\frac{e}{(4 \pi)^{2} \epsilon}\left[\alpha_{1} \hat{R}_{\mu \nu}^{2}+\alpha_{2} \hat{R}^{2}+\alpha_{3} \kappa^{4}\left(\bar{\eta} \gamma_{\mu} D_{\nu} \eta\right)^{2}+\alpha_{4} \kappa^{4}\left(\bar{\eta} \gamma^{\mu} D_{\mu} \eta\right)^{2}\right. \\
+\alpha_{5} \kappa^{4} \hat{R}_{\mu \nu \rho \sigma}\left(\bar{\eta} \sigma^{\mu \nu} \eta\right)\left(\bar{\eta} \sigma^{\rho \sigma} \eta\right)+\alpha_{6} \kappa^{2} \hat{R}^{\mu \nu}\left(\bar{\eta} \gamma_{\mu} D_{\nu} \eta\right) \\
+\alpha_{7} \kappa^{8} \prod_{i=1}^{4}\left(\bar{\eta} F_{i} \eta\right)+\alpha_{8} \kappa^{10}\left(\bar{\eta} D_{\nu} \eta\right)^{2}\left(\bar{\eta} F_{5} \eta\right)^{2} \\
\left.+\alpha_{9} \kappa^{4}\left(\left(\bar{\eta} F_{6} \eta\right)\left(\bar{\eta} F_{7} \eta\right)\right)^{\mu \nu}\left(\bar{\eta} \gamma_{\mu} D_{\nu} \eta\right)+\cdots\right]
\end{array}
$$

(Our splitting of the fermion fields differs from that used in ref. [42]; here, we choose $\psi=\eta+\chi$. So, $\eta$ and $\bar{\eta}$ are the fermion background fields.) The $F_{i}$ 's are expressions which 
contain $\gamma$-matrices, but no derivatives. (Note that the $F_{i}$ 's may include suppressed indices. For example, the raised indices in the $\alpha_{9}$ term are shared in some way between $F_{6}$ and $F_{7}$.) As usual, for on-shell amplitudes, we can implement the field equations:

$$
\begin{aligned}
& R_{\mu \nu}=\kappa^{2} T_{\mu \nu} \\
& R=0 \\
& \gamma^{\mu} D_{\mu} \eta=0 \\
& T_{\mu \nu} \sim \bar{\eta} \gamma_{\mu} D_{\nu} \eta .
\end{aligned}
$$

These imply that the $\alpha_{2}$ and $\alpha_{4}$ terms vanish on-shell, and that the $\alpha_{1}, \alpha_{3}$ and $\alpha_{6}$ terms are all equivalent. The only term which neither vanishes nor is mixed with others due to these equations is the $\alpha_{7}$ one. For this reason Deser and van Nieuwenhuizen focussed on this part of the counter-Lagrangian. Their method was to considered the diagrams described by fig. 3.A. Power counting implies that the only parts of (3.17) which can act as counterterms for this diagram are the $\alpha_{7}$ and $\alpha_{8}$ terms. (The other parts have divergences which will introduce too many powers of momenta.) It can be shown that the latter will only be,relevant if we include antisymmetric components of the vierbein in our calculation. It is easy to exclude these contributions. (In fact, we can define the vierbein in a symmetric (way and ignore them completely.) So, we find that we can relate the $\alpha_{7}$ term to this simple set of calculations. In ref. [42] the relevant calculation was considered and it was shown that $\alpha_{7}$ must be non-zero; there must be a term in the counter-Lagrangian

$$
\sim \frac{e}{(4 \pi)^{2} \epsilon} \kappa^{4}\left(\left(\bar{\eta} \gamma^{a} \gamma_{5} \eta\right)\left(\bar{\eta} \gamma^{b} \gamma_{5} \eta\right) \eta_{a b}\right)^{2}
$$

Clearly, knowledge of the existence of this term is all we require to deduce that the Dirac-Einstein system is neither finite nor renormalisable (it can not be absorbed into the Lagrangian or removed by the field equations). Fermions coupled to gravity do not solve the problems with the theory. (We should note that this calculation was restricted to the theory based on the second order formalism for the spin connection. The situation for a theory based on the first order formalism remains an open problem.) 

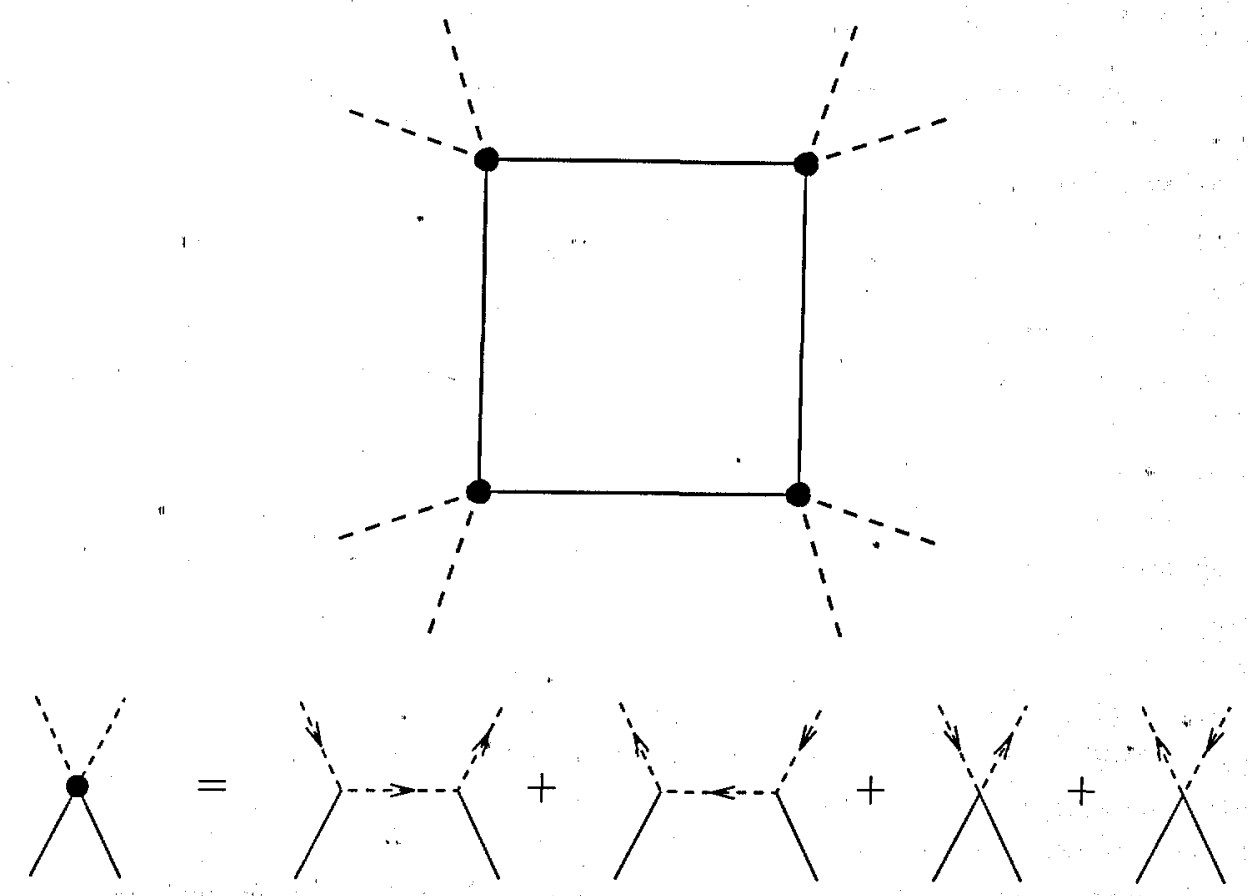

Figure 3.A: The diagram calculated by Deser and van Nieuwenhuizen.

The bottom figure shows the combination of vertices which the 'dot' represents

So, various calculations have lead to the conclusion that pure gravity is finite at one-loop (on-shell), but that this does not generalise to simple cases with a coupling to matter. To overcome this problem, we must look to theories in which the matter fields are included in the same SUSY multiplet as the graviton, as we discuss below.

Before we look at the situation at two-loops and in SUGRA, let us make a small detour to consider where the one-loop divergences in these theories will appear. We can show that infinities will not be seen in amplitudes with only external gravitons. We follow the proof of Grisaru et al [73]. For a general set of matter fields, $\phi^{i}$, we expect to have a counter Lagrangian of the form:

$$
\Delta L=\frac{1}{(4 \pi)^{2} \epsilon}\left(\alpha\left(R_{\mu \nu}\right)^{2}+\beta R^{2}+\gamma R_{\mu \nu \rho \sigma} M^{\mu \nu \rho \sigma}\left(\phi^{i}, e\right)+\delta N\left(\phi^{i}, e\right)\right)
$$

Where $M$ and $N$ are polynomials of the $\phi^{i}$ fields and their derivatives. (The vierbein, $e_{\alpha}^{\mu}$, enters since the derivatives must be covariant.) 
As in the case of pure gravity, we can always insert the field equations for the background field into our counter-Lagrangian. The general form for these is

$$
R_{\alpha \beta}-\frac{1}{2} g_{\alpha \beta} R=\kappa^{2} T_{\alpha \beta}
$$

Contracting the indices allows us to deduce that

$$
R=-\kappa^{2} T_{\alpha \beta} g^{\alpha \beta} \equiv-\kappa^{2} T
$$

and that

$$
R_{\alpha \beta} \doteq \kappa^{2}\left(T_{\alpha \beta}-\frac{1}{2} g_{\alpha \beta} T\right)
$$

The counter-Lagrangian can then be recast as

$$
\Delta L=\frac{1}{(4 \pi)^{2} \epsilon}\left(\kappa^{4} \alpha\left(T_{\mu \nu}\right)^{2}+\kappa^{4} \beta T^{2}+\gamma R_{\mu \nu \rho \sigma} M^{\mu \nu \rho \sigma}\left(\phi^{i}, e\right)+\delta N\left(\phi^{i}, e\right)\right)
$$

We can find the tree-level results (which is all we need at this stage) by inserting iterative solutions of the field equations:

$$
\begin{aligned}
& e_{a \mu}(x)=e_{a \mu}^{i n}(x)+\kappa \int D\left(x-x^{\prime}\right) J\left(\phi^{i}, e\right) d x^{\prime} \\
& \phi^{i}(x)=\phi_{i n}^{i}(x)+\kappa \int D\left(x-x^{\prime}\right) K\left(\phi^{i}, e\right) d x^{\prime}
\end{aligned}
$$

into $\Delta L$. Collecting the relevant number of 'in-fields' will give the terms which contribute to the $S$ matrix for any particular process. Note from equation (3.24) that there are no purely gravitational terms, implying that it is not possible to find non-trivial terms with only external gravitons.

This is a completely general result - we can always eliminate the Ricci quantities from the counterterms in favour of the energy-momentum tensor using the field equations. This will be relevant both to the results of our calculations and the discussion of SUGRA divergences below. 


\subsection{Two-Loop Divergences}

The finiteness of pure gravity at one-loop led to the hope that this might also be the case at higher orders. Unfortunately, a problem occurs as soon as we look at the twoloop counter-terms. There is one independent counter-term which cannot be dismissed for on-shell gravitons

$$
R_{\mu \nu}^{\sigma \rho} R_{\alpha \beta}^{\mu \nu} R_{\sigma \sigma \rho}^{\alpha \beta} .
$$

(By 'independent' we mean that any other possibilities can be related to this one.)

The only way for amplitudes to be divergence-free at this order is if the coefficient of this term vanishes. A number of calculations have shown that this is not the case [74]. We find that we have the two-loop counter-term:

$$
\Delta L=\frac{209}{2880} \frac{\kappa^{2}}{(4 \pi)^{4} \epsilon} R_{\mu \nu}^{\sigma \rho} R_{\alpha \beta}^{\mu \nu} R_{\sigma \rho}^{\alpha \beta}
$$

So, not only does gravity coupled to matter diverge at one-loop, but pure gravity is not much better - diverging at the two-loop level.

\subsection{SUGRA}

As we have stated, SUGRA theories contain a significant number of symmetries and our hope is that these symmetries will completely remove the problems of divergences in theories including gravity. We can show that this hope is realised at low orders.

We can use the symmetries in SUSY theories to relate the amplitudes for different sets of external particles (see appendix IV). We can use these 'SUSY Ward identities' [22,23,2] to deduce that there are no divergences at one-loop in SUGRA theories [73]: First, we note that these relations imply that all non-zero amplitudes within a SUSY multiplet are equal up to a phase. This phase cannot introduce a divergence into an amplitude, so if we can find one finite amplitude in a particular multiplet then we know that all amplitudes in that multiplet will be finite. Recall that we were able to show that one-loop amplitudes with external gravitons were finite in all cases. So, any amplitudes between particles in 
the same multiplet as the graviton are finite and, consequently, any theories which contain only gravity multiplets will be finite at one-loop order. Unfortunately, this fact does not carry over to theories also containing pure matter multiplets [75].

We do find, however, that for theories with pure gravity multiplets the finiteness goes further - all two-loop divergences also vanish. We can show this in a similar way to the above [76]; the two loop counterterm

$$
R_{\mu \nu}^{\sigma \rho} R_{\alpha \beta}^{\mu \nu} R_{\sigma \rho}^{\alpha \beta}
$$

represents a flipping of graviton helicity [77]. We can show using the SUSY Ward identities that supersymmetry does not allow this flipping, so this term must vanish. Another way of looking at this is to notice that it is not possible to adjust this term, or add other contributions to it, in such a way as to create a SUSY invariant quantity [78]. This implies that it cannot be a counterterm in a SUSY theory. So, at two loops our hopes about SUGRA seem to be justified; at the very least the extra symmetries have postponed the onset of divergences.

The situation becomes more complicated at three-loops. It has been shown that it is possible to construct counterterms at this order which obey all the necessary symmetries [78]. This would suggest that divergences are theoretically possible at this order. There may, of course, be some hidden symmetry which causes these to vanish, but divergence in two-loop pure gravity has led to pessimism regarding this. However, as with that case, the only true test is to carry out explicit calculations. The complexity of such calculations in PQG and SUGRA has inhibited investigations which may shed light on this. It is to new ways of carrying out such calculations that we begin to look in the next chapter. 
I don't want reality,

I want magic.

Vivien Leigh in 'A Streetcar named Desire'

\section{String-Based Rules I: QCD}

\subsection{Introduction}

As we have said, calculations in field theories are typically computationally complex. While the basic theory of the conventional Feynman techniques for these calculations is fairly straight-forward, its application soon leads to very complex calculations. In the Introduction, we discussed the incongruity of the complexity of $\mathrm{QCD}$ and gravity calculations with the simplicity of the answers. This leads us to the obvious questions: 'Isn't there a better approach to field theory calculations?' and, if so, 'Where do we look for this new approach?'

When comparing the processes of perturbative calculation in point-particle field theory. (using Feynman techniques) and string theory we notice that the latter has a number of advantages over the former:

- In Feynman calculations there are typically a large number of diagrams which must be considered at each order, in string theory there is only one;

- In string theory one loop amplitudes can be the formulated in such a way that the loop momenta are implicitly integrated out, in the Feynman diagram method this integration must be done explicitly;

- Simplifications due to spinor helicity techniques can be used at an early stage in string theory, whereas most can only be used at the end in field theory;

- Symmetries (such as SUSY) seem to simplify the calculation process in string theory rather than merely producing cancellations at the end. 
These hint that string theory could be a promising place to look for alternative methods of calculations to the traditional Feynman diagram methods; if we could find a relation between the results of string calculations and field theory amplitudes then we could carry some or all of these advantages over.

In fact, it can be proved that string theories contain gauge field theories in the infinitetension limit $[12,13]$. Hence, we have a clear connection between the theories with simpler calculation techniques and the theories for which we would like results. This connection was exploited by Bern and Kosower to develop a new, simpler calculation technique for one-loop QCD [3,4]. The result was a straightforward set of rules which could be used easily without knowledge of the development details. These rules have successfully been use to carry out one-loop 4- and 5-gluon amplitudes $[3,4,25]$ and many of the techniques derived from the rules have been used in electroweak calculations [24]. Since gravity is also contained in the infinite-tension limit of certain string theories [12], it is natural to try to apply the same techniques to this more complex case. The results from such an attempt are even more dramatic than the QCD case. The first steps in this application were made by Bern, Dunbar and Shimada in ref. [31]. We will present a complete discussion in the next chapter.

In this chapter we will give a brief description of the relevant elements of string theory and the infinite-tension limit, followed by a discussion of the way the rules for QCD are derived. As would be expected these rules are simpler than those for gravity. However, many of the significant ideas are shared between the two sets, and an introduction to these in the simpler case will aid the later discussion. We finish with a summary of the QCD rules and an example of calculations using this method.

The 'string-inspired' rules for QCD were originally developed using a heterotic string theory [3]. This allowed a direct derivation of a complete set of rules from a fully consistent string in four dimensions. We will concentrate on an alternative derivation using bosonic 
strings $[15,14]$. Bosonic string theory is one of the simplest available and, hence, engenders the simplest derivation and a particularly compact form of the field theory rules. There are potential problems in using bosonic string theories as our starting point - they do not contain all necessary particles and are formally inconsistent in four dimensions - but a correct and complete set of rules can be constructed by comparison with the heterotic-based result. For theories containing particles of spin less than or equal to one (e.g. QCD, QED) the formulation can be carried out using an open string theory. (For the case of perturbative gravity, where the gauge boson has a spin of 2 , we must look at closed string theories. There are both obvious and subtle differences in the construction of these theories which must be taken into account while developing field theory rules from them.)

\subsection{Amplitudes in String Theory ${ }^{\dagger}$}

It is well-known that string theory models particles as excitations of one-dimensional objects - 'strings'. Strings can either be closed or open; i.e., they have two free ends or they are loops with no free ends, respectively (fig. 4.A).
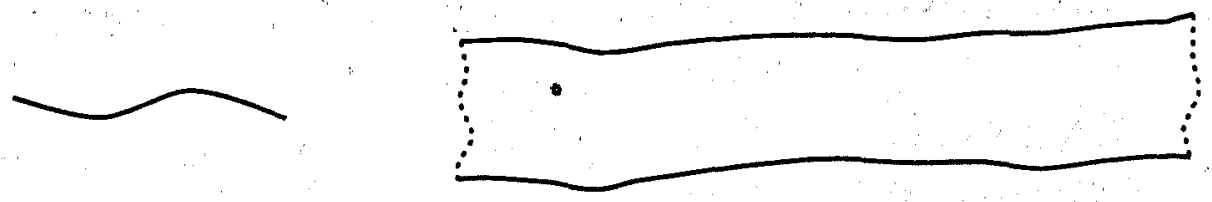

(a)
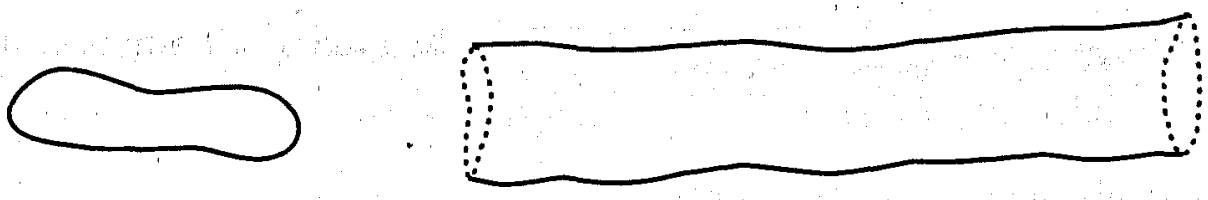

(b)

Figure 4.A: (a) Open and (b) closed strings and example world-sheets for each freely propagating.

All particle trajectories in a string theory are represented by two-dimensional 'worldsheets'; the area mapped out by the strings as they move through space (fig. 4.A). We

\footnotetext{
See, for instance, ref. [34] and references therein.
} 
parameterise the world-sheets with a space-like and a time-like parameter, $\sigma$ and $\tau$. We can then describe the string trajectory mathematically by $X^{\mu}(\sigma, \tau)$, a vector giving the space-time position of the string at world-sheet position $(\sigma, \tau)$. We also introduce a metric, $h_{\mu \nu}(\sigma, \tau)$, on the world-sheet. This allows us to write the action of the free string as

$$
S=\left(4 \pi \alpha^{\prime}\right)^{-1} \int d^{2} \xi \sqrt{h} h_{\alpha \beta} \partial^{\alpha} X_{\mu} \partial^{\beta} X^{\mu}
$$

(Note that the integral and the $\alpha$ and $\beta$ indices are on the two-dimensional world-sheet; the $\mu$ index is on the physical space.)

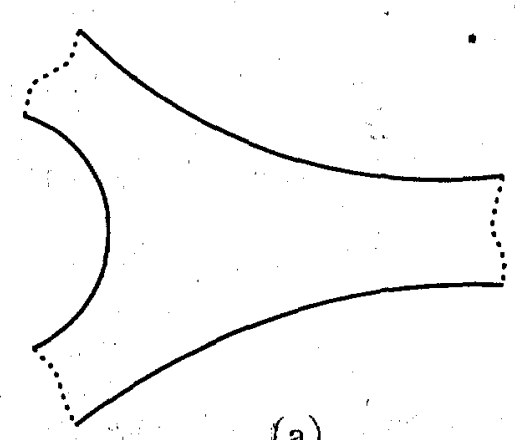

(a)

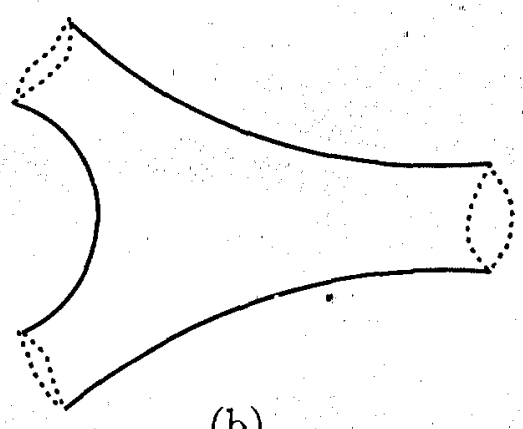

(b)

Figure 4.B: World-sheets representing the joining of two strings to form a third for (a) open and (b) closed strings

An interaction in string theory can be pictured as the world sheet in fig. 4.B; i.e.; those which describe the joining of two strings to form one new string (or, equivalently, one string splitting into two). We find the string diagrams for a perturbative expansion in an analogous way to point-particle theory: The interactions are used as the building blocks for diagrams with the required perturbative order and number of external particles. In the case of open strings, the result will be world-sheets with a number of 'holes' and 'strips' extending to the far past and far future. The holes correspond to loops in Feynman diagrams and the strips to external particles. In the same way, for closed strings we will have 'handles' (corresponding to loops) and 'tubes' extending to the past and future (corresponding to external particles). 

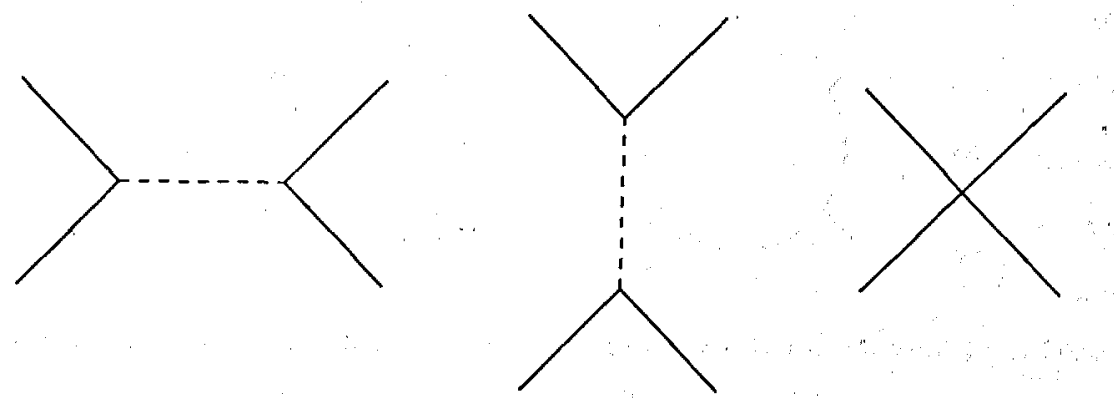

Figure 4.C: Contributions to four-point tree amplitude in. point particle theory.

The major difference between point-particle and string theory (as far as perturbation theory goes) is that the latter.has only one diagram at each perturbative order; since manifolds are completely determined by the number of loops and holes they have, we need only consider diagrams which are topologically distinct. There is also no need to consider diagrams with different internal particles propagating since all particles described by the string are included. For example, consider a simple 4-point tree amplitude. In Feynman calculations we would potentially require all the diagrams in fig 4.C, including a sum over all particles in the theory propagating along the internal lines. In string theory all these possibilities are included in one tree diagram (fig. 4.D). The different contributions will be found as different regions of integration in the string amplitude.

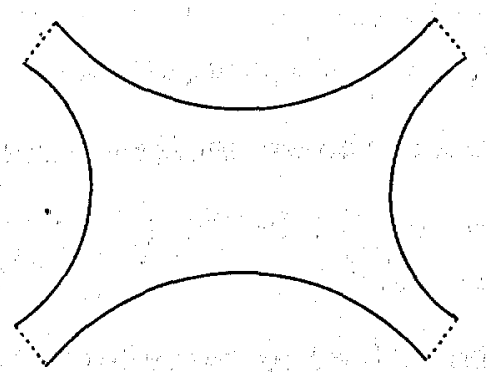

Figure 4.D: Contribution to four-point tree amplitude in string theory.

To begin calculating string amplitudes in practice, we make use of a conformal invariance of the string action to manipulate the diagrams into a more manageable form. We can make a conformal rescaling of the world-sheet metric which takes the world-sheets to 

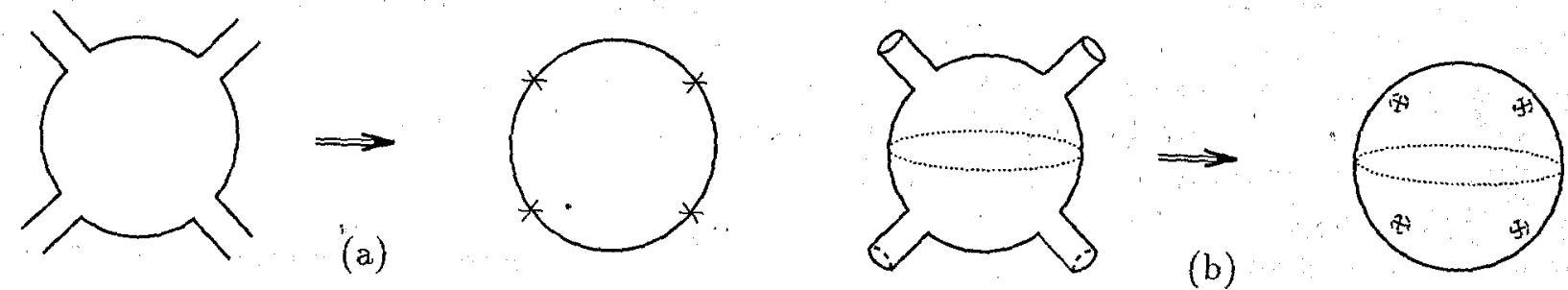

Figure 4.E: Conformal mappings taking the external strings of a tree to points on a (a) disc and (b) sphere.
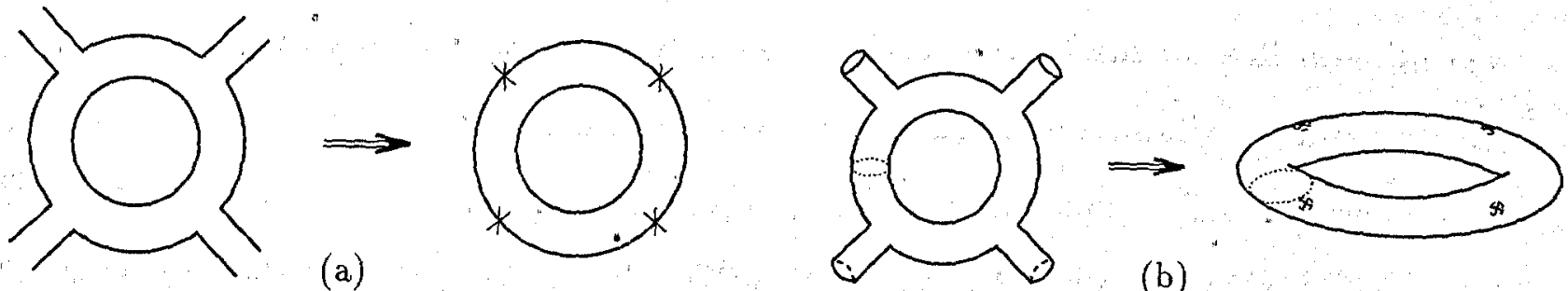

(b)

Figure 4.F: Conformal mapping taking a one-loop diagram to points on (a) an annulus and (b) a torus.

compact manifolds with the external particles mapped to points on the surface (e.g. see figs. 4.E and 4.F). We are interested here in one loop amplitudes (fig. 4.F): In the case of the open string, this mapping takes a one loop diagram to an annulus with vertex operators distributed over the two edges. Similarly, for a closed string we obtain a torus with vertex operators distributed over its surface. Since this process maps external states to points, the quantum numbers for these must be retained in some way. We find that there are local operators - 'vertex operators' - at each point encoding this information. For a particle, $\Lambda$, with momentum $k_{\mu}$, the vertex operators are of the form

$$
V_{\Lambda}(k)=\int d \nu \sqrt{h} W_{\Lambda}(\nu) e^{i \alpha^{\prime} k \cdot X}
$$

$W_{\Lambda}$ contains the quantum number information for the particle (ie the spin dependence, etc). The integral is over the locations of emission points on the world-sheet. $X^{\mu}$ and $h_{\mu \nu}$ are the same quantities as those found in the action (4.1). $\alpha^{\prime}$ is a string parameter which we can identify as the reciprocal of the string tension.

One way in which we can express an amplitude using the vertex operators is via the Polyakov formalism, in which the amplitude is the expectation value of the vertex operators 
summed over all possible string topologies:

$$
A_{n}\left(\Lambda_{1}, k_{1} ; \ldots ; \Lambda_{n}, k_{n}\right)=\sum_{\text {topologies }}\left\langle\prod_{i=1}^{n} V_{\Lambda_{i}}\left(k_{i}\right)\right\rangle .
$$

where the average is weighted as

$$
\left\langle V_{1} \ldots V_{n}\right\rangle \sim \int[\mathrm{D} X]\left[\mathrm{D} h_{\alpha \beta}\right] \exp (-S) V_{1}\left(k_{1}\right) \ldots V_{n}\left(k_{n}\right)
$$

and $S$ is the string action defined earlier (4.1). This is the form we will use. In oneloop calculations the functional integral over the metric is essentially an integral over conformally inequivalent tori (or annuli), specified by a parameter $\tau$. The metric on each of these tori (annuli) is $h_{\alpha \beta} \sim \eta_{\alpha \beta}$. So, $\int\left[\mathrm{D} h_{\alpha \beta}\right]$ will be reduced to $\int \mathrm{d} \tau f(\tau)$. In order to manipulate this into a more convenient form for calculations, we can use Wick's Theorem to re-write the average. The most useful expression of this for our purposes is (from appendix 7.A of ref. [34])

$$
\left\langle: e^{A}:: e^{B}:\right\rangle=e^{\langle A B\rangle}
$$

which can be generalised as

$$
\left\langle: e^{A_{1}}:: e^{A_{2}}: \cdots: e^{A_{M}}:\right\rangle=\prod_{i<j} e^{\left\langle A_{i} A_{j}\right\rangle} .
$$

Thus, if we can express the $V$ 's in the form $V=\int d^{2} \nu e^{B}(4.3)$ will become (in the one-loop case)

$$
A_{n}^{1-\text { loop }} \sim \int d^{2} \tau f(\tau) \prod_{k} \int d^{2} \nu_{k} \prod_{i<j} e^{\left(B_{i} B_{j}\right\rangle}
$$

This will be our starting point for the development of the Bern and Kosower method. We will look at the infinite tension limit of this expression to construct a new set of rules for one-loop calculations.

Before we go on to look at the effect of this limit, we must make a short comment about the dimension in which our strings live. It is well known that bosonic string theories are only consistent in a 26 dimensional space-time. However, we are interested in amplitudes 
in $4-2 \epsilon$ dimensions (working in a dimensional regularisation scheme). This might appear to be a problem, but, for our purposes, we find that we can simply truncate the number of dimensions in a naive way from 26 to $4-2 \epsilon$ with only a minor adjustment. We can easily confirm that this is sufficient for the purpose of our analysis by comparing the results with the fully consistent heterotic string construction.

\subsection{The Infinite Tension Limit ${ }^{\dagger}$}

In order to obtain the particle amplitudes required we must consider the field theory or 'infinite tension' limit of our string amplitude $[12,13,3]$. The conventional string parameter $\alpha^{\prime}$ is the reciprocal of the string tension, so 'infinite tension' corresponds to taking

$$
\alpha^{\prime} \rightarrow 0
$$

We must look carefully to find all the contributions in this limit: in both the open and string cases we find that the amplitude has an overall factor of $\left(\alpha^{\prime}\right)^{m}(m>0)$, so we must look at the regions of $\tau$ and $\nu$ integration which produce compensating powers of $\left(\alpha^{\prime}\right)^{-1}$. We will see that there are two regions which must be considered. The first is where the position of two vertex operators come close - or are 'pinched'. i.e.

$$
\left|\nu_{i}-\nu_{j}\right| \rightarrow 0 \quad \text { some } i, j
$$

$\left(\nu_{i}\right.$ and $\nu_{j}$ are the positions of two emission points, cf. (4.2).) In the infinite tension limit, this corresponds to extracting massless poles. We can regard it 'diagrammatically' as two external legs coming together to produce a tree attached to the loop (fig. 4.G). Each pinch of this form will contribute a leading $\left(\alpha^{\prime}\right)^{-1}$ to the amplitude expression. In practice we will find that we must consider all possible combinations of pinches. These combinations give us a set of diagrams which must be considered - all one-loop $\phi^{3}$ diagrams with the correct number of external particles. This may seem surprising since the theories we are

$\uparrow$ This section follows ref. 3 closely. 

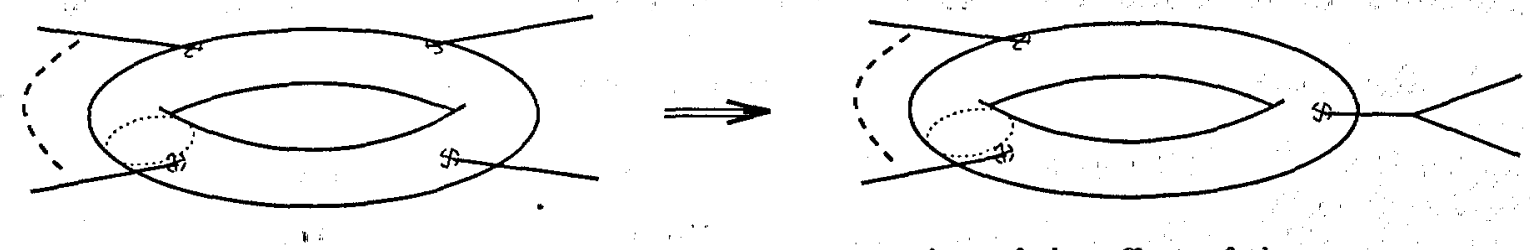

Figure 4.G: A diagrammatic representation of the effect of the limit $\left|\nu_{i}-\nu_{j}\right| \rightarrow 0$.

considering have vertices where more than 3 particles meet. In fact, gravity has n-point vertices for all $n \geq 3$. This reduction of vertices to be considered is a simplification reflecting the string theory roots.

The second integration region is the one in which

$$
\tau \rightarrow \infty
$$

(In the closed string case this will translate to $\Im(\tau) \rightarrow \infty$.) 'Diagtammatically' we can think of this as shrinking the annulus or torus to a thin circular wire (fig. 4.H). We find that this limit leads ús to consider an integral of the form

$$
\int^{\infty} d \tau(\tau)^{m+\epsilon / 2} e^{-\alpha^{\prime} K \tau}
$$

for some $m \geq 0$. This gives a contribution (to leading order in $\alpha^{\prime}$ ) of

$$
\frac{\Gamma(m+1+\epsilon / 2)}{\left(\alpha^{\prime} K\right)^{m+1+\epsilon / 2}}
$$

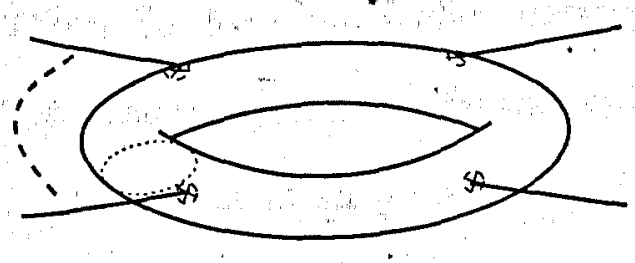

Figure 4.H: A diagrammatic representation of the effect of the limit $\tau \rightarrow \infty$. 
So, we have $\left(\alpha^{\prime}\right)^{-1}$ contributions from the $\tau$ integration and from combinations of pinches. We might wonder if these all give non-zero contributions as $\alpha \rightarrow 0$. Let us consider the open string case: Here we have an overall factor of $\left(\alpha^{\prime}\right)^{n-2}$ ( $\mathrm{n}$ is the number of external legs). Whatever choice of 'pinches' we are considering, the combination of these two contributions cancels these $n-2$ powers completely; the pinches give $\left(n-n_{l}\right)$ and the loop integration $\left(n_{l}-2\right)$ powers of $\left(\alpha^{\prime}\right)^{-1}\left(n_{l}\right.$ is the number of legs attached to the loop). In the closed string case the situation is slightly more complicated but essentially the same. In both cases, the implication is that we must include all pinch combinations ${ }^{\ddagger}$, and that these give the complete non-vanishing contribution as $\alpha^{\prime} \rightarrow 0$.

After considering the $\tau$ integration we are left with the integrals over the remaining $\nu_{i}$ 's. As the $\nu_{i}$ 's always appear with $1 / \tau$ and $0<\nu_{i}<\tau$, the only region of $\nu_{i}$ integration with non-zero contribution in this limit is

$$
\left|\nu_{i}\right| \rightarrow \infty \quad \text { s.t. } \frac{\nu_{i}}{\tau} \neq 0 \quad \forall i
$$

(In the case of closed strings it is the limit in which the imaginary part of these quantities goes to infinity which must be considered.) We will find that these amount to Feynman parameter integrals. ( $\mathrm{K}$ in equation (4.12) will be seen to be a typical Feynman parameter denominator.) The numerator of the integral will depend on the explicit form of the vertex operators and the particle content of the theory.

It is the $\alpha^{\prime} \rightarrow 0$ limit which allows us to control the particle content. The limit causes all massive states to decouple so that we are left only with the massless states and the tachyon. So, for a particular spectrum, we merely select theory with a suitable massless sector. The tachyon is a potential problem; we do not want it to feature in our final results, but it is present in all bosonic theories and produces exponentially large contributions in the limit (4.8). In fact, since it is clear which contributions come from the tachyon, it is $\ddagger$ We omit tadpole diagrams and those with the loop isolated on an external leg. These vanish because
we implement the usual dimensional regularisation prescription [13]. 
sufficient for us merely to drop these as we go along. As with the truncation of dimension we need not worry since the final results can be shown to work.

So, we begin to see the form thàt our method must take: we expect to look for a number of $\phi^{3}$ diagrams corresponding to pinch contributions, plus a set of rules which lead to a Feynman parameter integral for each diagram. Summing over all the contributions will give us an expression for the amplitude.

\subsection{Derivation of QCD Rules from Open Strings [14,15]}

We now turn to an explicit discussion of this limit in the open bosonic string case. We know from earlier in this chapter that amplitudes in this theory can be described by a set of vertex operators sitting on the boundaries of an annulus.

Open bosonic string theory has a massless sector which contains only a spin one YangMills particle, so in the first instance we can use it to produce rules for use in pure QCD gauge calculations. Note that amplitudes in QCD must include traces of colour factors, so before discussing the rules let us see how these would enter in the string-inspired method. There is a very simple way of expressing these amplitudes which comes out naturally from open string theory:

$$
\begin{gathered}
A_{n}=g^{n} \sum_{\sigma \in S_{n} / S_{n ; 1}} N_{c} \operatorname{Tr}\left(T^{a_{\sigma(1)}} \ldots T^{a_{\sigma(n)}}\right) A_{n ; 1}\left(k_{\sigma(1)}, \epsilon_{\sigma(1)} ; \ldots ; k_{\sigma(n)}, \epsilon_{\sigma(n)}\right) \\
\quad+g^{n} \sum_{m=2}^{[n / 2]} \sum_{\sigma \in S_{n} / S_{n ; m}} \operatorname{Tr}\left(T ^ { a _ { \sigma ( 1 ) } } \ldots T ^ { a _ { \sigma ( m - 1 ) } ) } \operatorname { T r } \left(T^{a_{\sigma(m)}} \ldots T^{\left.a_{\sigma(n)}\right)}\right.\right. \\
\times A_{n ; m}\left(k_{\sigma(1)}, \epsilon_{\sigma(1)} ; \ldots ; k_{\sigma(n)}, \epsilon_{\sigma(n)}\right)
\end{gathered}
$$

where the traces are over colour matrices. This is a 'colour decomposition' of QCD amplitudes [79]. In this method, rather than calculating the full amplitude, $A_{n}$, we need only calculate the simpler 'colour-ordered' sub-amplitudes, $A_{n ; m}$. In the field theory limit of open string theory this occurs naturally due to Chan-Paton [80] factors of the string (these are quantum numbers which sit on the ends of the string). These factors produce the traces 
seen in the above sum. We know that vertex operators sit on the edges of an annulus. If all vertex operators are located on one edge of annulus then we have one trace; terms with two traces occur when vertex operators are shared out between the two boundaries. Since our aim here is to give pointers to the closed string development for gravity we will not discuss this further: Closed strings do not have Chan-Paton factors and the world-sheets do not have edges; these differences are consistent with our understanding of gravity since it has no equivaleft to colour and we expect to have to sum over all orderings. Instead, we concentrate on the calculation of $A_{n ; 1}$ since this is closest in form to the approach for gravity.

For the calculation of $A_{n ; 1}$ we must consider an open string amplitude with all vertex operators located on the same boundary of the annulus (as in fig. 4.I). Since the operators are restricted to one-dimensional spaces, there is an implicit ordering of the vertex operators. (It is this ordering which allows us to use Wick's theorem.) We must integrate over all positions of the vertex operators consistent with this ordering.

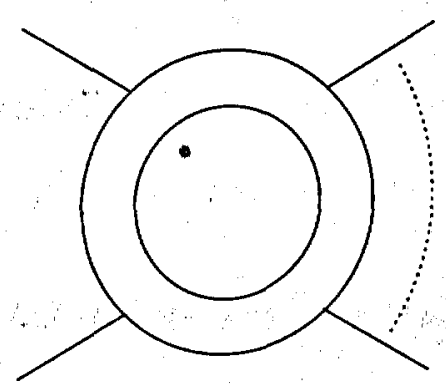

Figure 4.I: The string diagram relevant for the calculation of $A_{n ; 1}$.

For external vector particles, the vertex operators required are

$$
\begin{aligned}
V_{i} & \sim \frac{g}{\sqrt{\alpha^{\prime}}} \epsilon_{i} \cdot \partial_{\nu_{i}} X\left(\nu_{i}\right) e^{\left(i \sqrt{\alpha^{\prime}} k_{i} \cdot X\left(\nu_{i}\right)\right)} \\
& \left.\sim \frac{y}{\sqrt{\alpha^{\prime}}} e^{i\left(\sqrt{\alpha^{\prime}} k_{i} \cdot X+\epsilon_{i} \cdot \partial X\right)}\right|_{\text {linear }}
\end{aligned}
$$

where, by 'linear', we mean 'keep only terms linear in $\epsilon_{i}$ '. Since we have $\nu_{i}$ in a suitable 'exponentiated' form we know from section 4.2 that we can express the amplitude as

$$
\left.A_{n}^{1-\text { loop }} \sim \int \prod d \nu_{k} \prod_{i<j} e^{\left\langle\left(\sqrt{\alpha^{\prime}} k_{i} \cdot X+\epsilon_{i} \cdot \partial X\right)\left(\sqrt{\alpha^{\prime}} k_{j} \cdot X+\epsilon_{j} \cdot \partial X\right)\right\rangle}\right|_{\text {multi-linear }}
$$


'Multi-linear' is the natural generalisation of the condition in (4.15) - we keep all terms containing one of each $\epsilon_{i}$. We can expand a generic term in the product as

$$
\begin{array}{r}
\exp \left(\left\langle\left(i \sqrt{\alpha^{\prime}} k_{i} \cdot X\left(\nu_{i}\right)+\epsilon_{i}: \partial_{\nu_{i}} X\left(\nu_{i}\right)\right)\left(i \sqrt{\alpha^{\prime}} k_{j} \cdot X\left(\nu_{j}\right)+\epsilon_{j} \cdot \partial_{\nu_{j}} X\left(\nu_{j}\right)\right)\right\rangle\right) \\
=\exp \left(\alpha^{\prime} k_{i}^{\mu} k_{j}^{\nu}\left(X_{\mu}\left(\nu_{i}\right) X_{\nu}\left(\nu_{j}\right)\right\rangle+\sqrt{\alpha^{\prime}} k_{i}^{\mu} \epsilon_{j}^{\nu}\left\langle X_{\mu}\left(\nu_{i}\right) \partial_{\nu_{j}} X_{\nu}\left(\nu_{j}\right)\right\rangle\right. \\
\left.+\sqrt{\alpha^{\prime}} k_{j}^{\mu} \epsilon_{i}^{\nu}\left\langle\partial_{\nu_{i}} X_{\mu}\left(\nu_{i}\right) X_{\nu}\left(\nu_{j}\right)\right\rangle+\epsilon_{j}^{\mu} \epsilon_{i}^{\nu}\left\langle\partial_{\nu_{i}} X_{\mu}\left(\nu_{i}\right) \partial_{\nu_{j}} X_{\nu}\left(\nu_{j}\right)\right\rangle\right)
\end{array}
$$

a function of the tvo-point correlation function. This correlation function can be evaluated as

$$
\begin{aligned}
\left\langle X_{\mu}\left(\nu_{i}\right) X_{\nu}\left(\nu_{j}\right)\right\rangle & =\delta_{\mu \nu} G_{B}\left(\nu_{i}-\nu_{j}\right) \\
& =-\delta_{\mu \nu}\left(\log \left|2 \sinh \left(\nu_{i j}\right)\right|-\frac{\left(\nu_{i j}\right)^{2}}{\tau}-4 q \sinh ^{2}\left(\nu_{i j}\right)\right)+\mathcal{O}\left(q^{2}\right)
\end{aligned}
$$

$\left(\nu_{i j}=\nu_{i}-\nu_{j}\right.$ and $\left.q=\exp -2 \tau\right)$. In general we will write this as $G_{B}$ since we will only require certain limits of the function. In this way (4.17) becomes,

$$
\left.\exp \left(\alpha^{\prime} k_{i} \cdot k_{j} G_{B}\left(\nu_{i j}\right)+\sqrt{\alpha^{\prime}}\left(k_{i} \cdot \epsilon_{j}-k_{j} \cdot \epsilon_{i}\right) \dot{G}_{B}\left(\nu_{i j}\right)-\epsilon_{i} \cdot \epsilon_{j} \ddot{G}_{B}\left(\nu_{i j}\right)\right)\right|_{\text {multi-linear }}
$$

with $\dot{G}_{B}(\nu)=\frac{1}{2} \partial_{\nu} G_{B}(\nu)$ and $\ddot{G}_{B}(\nu)=\frac{1}{4} \partial_{\nu}^{2} G_{B}(\nu)$. Inserting this in (4.16), and filling in the missing terms, gives us a complete expression for the $n$-point amplitude

$$
\begin{aligned}
& A_{n ; 1}=i \frac{(4 \pi)^{\epsilon / 2}}{16 \pi^{2}}\left(\mu^{\epsilon} g \sqrt{2}\right)^{n}\left(\alpha^{\prime}\right)^{n / 2-2} \int_{0}^{\infty} d \tau \int_{i=1}^{n-1} d \nu_{i} \theta\left(\nu_{i}-\nu_{i+1}\right) \tau^{-2+\epsilon / 2} q^{-1}[f(q)]^{-2\left(1-\delta_{R} \epsilon / 2\right)} \\
& \times\left.\prod_{i<j}^{n} \exp \left(\alpha^{\prime} k_{i} \cdot k_{j} G_{B}\left(\nu_{i j}\right)+\sqrt{\alpha^{\prime}}\left(k_{i} \cdot \epsilon_{j}-k_{j} \cdot \epsilon_{i}\right) \dot{G}_{B}\left(\nu_{i j}\right)-\epsilon_{i} \cdot \epsilon_{j} \ddot{G}_{B}\left(\nu_{i j}\right)\right)\right|_{\text {multi-linear }}
\end{aligned}
$$

where $f(q)$ is the partition function

$$
f(q)=\prod_{n=1}^{\infty}\left(1-q^{n}\right)
$$

and there is an arbitrariness in the $\nu_{i}$ 's which has allowed us to set $\nu_{n}$ equal to $\tau$. We emphasise again that the $\nu$ 's are implicitly ordered in this expression $\left(\tau=\nu_{n}<\nu_{n-1}<\right.$ $\cdots<\nu_{1}<1$ ). Note that, as discussed earlier, this is a naive truncation of the bosonic 
string expression from 26 to $4-2 \epsilon$ dimensions. The subtlety is that we keep the leading $q^{-1}$ by hand in order to obtain a sensible limit as $\tau \rightarrow \infty$.

We could use this expression 'as it is' as our starting point for the rules. However, before looking at the limits it is useful to remove all $\ddot{G}_{B}$ factors by integrating by parts. Doing this will enable us to find a particularly simple form of the rules. For each $\ddot{G}_{B}\left(\nu_{i j}\right)$ we integrate by parts with respect to one of the variables $\nu_{i}$ and $\nu_{j}$. For example, in a four point calculation a simple term would be one such as

$$
\ddot{G}_{B}\left(\nu_{12}\right) \dot{G}_{B}\left(\nu_{23}\right) \dot{G}_{B}\left(\nu_{34}\right)^{2} \exp \left(\alpha^{\prime} \sum_{i<j} k_{i} \cdot k_{j} G_{B}\left(\nu_{i j}\right)\right)
$$

We can remove $\ddot{G}_{B}\left(\nu_{12}\right)$ by integrating by parts w.r.t. $\nu_{1}$ to give ${ }^{\dagger}$

$$
\begin{aligned}
\alpha^{\prime} \dot{G}_{B}\left(\nu_{12}\right) \dot{G}_{B}\left(\nu_{23}\right) \dot{G}_{B}\left(\nu_{34}\right)^{2} \exp \left(\alpha^{\prime} \sum_{i<j} k_{i} \cdot k_{j} G_{B}\left(\nu_{i j}\right)\right) \\
\\
\quad \times\left(k_{1} \cdot k_{2} \dot{G}_{B}\left(\nu_{12}\right)+k_{1} \cdot k_{3} \dot{G}_{B}\left(\nu_{13}\right)+k_{1} \cdot k_{4} \dot{G}_{B}\left(\nu_{14}\right)\right)
\end{aligned}
$$

In many cases such a manipulation will create another $\ddot{G}_{B}$, but iteration of the process will eventually lead to an expression with no $\ddot{G}_{B}$ ' $\mathrm{s}^{\ddagger}$. (It was proved in ref. [79] that this process always ends.)

Having carried out the integration by parts we will be left with an integral over $\exp \left(G_{B}\right)$ and $\dot{G}_{B}$ 's. We can now look at the effect of $\alpha^{\prime} \rightarrow 0$. When (4.20) is expanded out fully, the leading power of $\alpha^{\prime}$ will be $\left(\alpha^{\prime}\right)^{n-2}$. So, to find the non-zero factors in the

† One might expect boundary terms to appear here, but we can dispatch these easily: All boundary terms will contain a factor of the form $\left.\left|\nu_{i}-\nu_{j}\right|^{-n-\alpha^{\prime} k_{i} \cdot k_{j}}\right|_{\nu_{i} \rightarrow \nu_{j}}$. (Some terms will require the use of periodicity $\nu \rightarrow \nu+\tau=\nu+\nu_{n}$ to enable them to be seen in this form.) With a suitable analytic continuation of $k_{i} \cdot k_{j}[13,79,81]$ these will vanish.

$\ddagger$ For example, if we had (unwisely) chosen to integrate (4.22) by parts w.r.t. $\nu_{2}$ we would have obtained

$$
\dot{G}_{B}\left(\nu_{12}\right) \dot{G}_{B}\left(\nu_{34}\right)^{2} \exp \left(\alpha^{\prime} \sum_{i<j} k_{i} \cdot k_{j} G_{B}\left(\nu_{i j}\right)\right)\left(\alpha^{\prime} \sum_{i=2}^{4} k_{1} \cdot k_{i} \dot{G}_{B}\left(\nu_{1 i}\right) \dot{G}_{B}\left(\nu_{23}\right)-\ddot{G}_{B}\left(\nu_{23}\right)\right)
$$

Integrating the the second term by parts w.r.t. $\nu_{3}$ followed by a further integration by parts w.r.t. $\nu_{4}$ will eventually give a $\ddot{G}_{B}$-free expression equivalent to (4.23). 
limit, we must look for places where inverse powers of $\alpha^{\prime}$ are produced to cancel this. As we discussed in section 4.3, there are two types of contribution, 'pinch' and 'loop', which we must consider.

The first of these comes from the integration regions where $\nu_{i} \rightarrow \nu_{j}$. In this limit the $\exp \left(\alpha^{\prime} \sum_{i<j} k_{i} \cdot k_{j} G_{B}^{i j}\right)$ term looks like $\nu^{-\alpha^{\prime} k_{i} \cdot k_{j} / \pi}$ and each $\dot{G}_{B}^{i j}$ produces a factor of $\nu_{i j}^{-1}$ (see appendix I). So in general we will have an integral of the form

$$
\int d \nu\left(\nu_{i j}\right)^{-n-\alpha^{\prime} k_{i} \cdot k_{j} / \pi} \quad n \geq 0
$$

In the $\alpha^{\prime} \rightarrow 0$ limit this will only produce a lasting contribution if $n=1$ :

$$
\int d \nu_{i} \frac{1}{\nu_{i j}^{1+\alpha^{\prime} k_{i} \cdot k_{j} / \pi}} \longrightarrow-\frac{\pi}{\alpha^{\prime} k_{i} \cdot k_{j}} \quad \alpha^{\prime} \rightarrow 0
$$

For $n \neq 1$ the resulting denominator will be ( $\alpha^{\prime}$ - integer) which will not survive $\alpha^{\prime} \rightarrow 0$ due to the the overall powers of $\alpha^{\prime}$. We can recognise (4.25) as a 'massless propagator' like term, confirming our earlier assertion that pinches extract massless poles.

The practical effect is that, when considering a pinch contribution in which $\nu_{i j} \rightarrow 0$ we will drop all those terms which do not have exactly one power of $\dot{G}_{B}\left(\nu_{i j}\right)$. Where single powers of $\dot{G}_{B}\left(\nu_{i j}\right)$ do occur they will be replaced by the massless pole $1 / k_{i} \cdot k_{j}$; $\nu_{i}$ will be substituted for $\nu_{j}$ in all other factors.

After carrying out a series of pinches we will have introduced a factor of $\left(\alpha^{\prime}\right)^{-n+n_{l}}$ ( $n_{l}$ is the number of legs still attached to the loop after pinching). We will also have reduced the number of $\nu$ integrations to $n_{l}$; we will now only need to integrate over a subset $\left\{\nu_{i_{1}}, \nu_{i_{2}}, \ldots, \nu_{i_{n_{l}}}\right\}$. (Note how this reduction of variables changes the exponential term,

$$
\exp \left(\alpha^{\prime} \sum_{l<m}^{n_{l}} P_{i_{l}} \cdot P_{i_{m}} G_{B}\left(\nu_{i_{l} i_{m}}\right)\right)
$$

its form is the same, but the momenta are now the momenta of the legs attached to the loop.) 
Once we have considered the pinch contributions we must look at the $\tau,|\nu| \rightarrow \infty$ limit. Note first that the string amplitude contains an overall of $q^{-1}$; an exponential function of $\tau$. In the $\tau \rightarrow \infty$ limit we must find a single power of $q$ to cancel this and produce finite contributions. Terms with higher powers of $q$ will vanish in the limit. (There is a component of the amplitude which has no $q$ 's to cancel this factor, leading to a exponentially growing term. This is the tachyonic contribution referred to above which can simply be discarded for our purposes.) There are two places in our expression where we can find this $q$; the partition function (4.21) and the Green functions (appendix II).

First let us look at the partition function. If we expand $f(q)$ it is clear that this will introduce an overall factor of $2\left(1-\frac{{ }^{\circ}}{2} \delta_{R} \epsilon\right)$, but have no other effect. We then only need the $\mathcal{O}\left(q^{0}\right)$ terms in the the $\dot{G}_{B}$ expansion (appendix II) leaving us with

$$
\dot{G}_{B}(\nu) \sim-\frac{1}{2} \operatorname{sign}(\nu)+\nu
$$

So, this contribution will simply consist of an overall factor plus the above substitution for each of the Green functions.

From (II.2) we see that every Green function also has a term including $q$ which could potentially contribute, so this is the second possibility which we must consider. This $\mathcal{O}(q)$ part of the Green functions is takes the form

$$
-q \exp \left(2 \tau\left|x_{i}-x_{j}\right|\right) \text {. }
$$

where we have defined $x_{i} \equiv \nu_{i} / \tau$. When considering this, we are in a similar situation to the overall $q^{-1}$; the term contributes an exponentially growing factor to the $\tau$ integral which we discard as part of the tachyon contribution, but it also produces finite contributions which we must take into account. The finite contributions must come where this term is multiplied by a factor which cancels the exponential growth exactly. In a general product of $\dot{G}_{B}$ 's, equation (4.28) will be multiplied be factors of $\exp (-2|\nu|)$ to give an overall contribution of

$$
-q \exp \left(2 \tau\left(\left|x_{i}-x_{j}\right|-\sum_{\text {pairs }(k, l)}\left|x_{k}-x_{l}\right|\right)\right)
$$


This can only give a finite (and non-zero) contribution if the sum exactly cancels $\left|x_{i}-x_{j}\right|$. This can only happen if the sum follows the ordering of the external legs and takes the form

$$
\begin{aligned}
\sum\left|x_{k}-x_{l}\right|=\left|x_{i}-x_{i_{1}}\right|+\left|x_{i_{1}}-x_{i_{2}}\right| & +\cdots \\
\cdots & +\left|x_{i_{r-1}}-x_{i_{r}}\right|+\left|x_{i_{r}}-x_{j}\right|
\end{aligned}
$$

which clearly cancels $\left|x_{i}-x_{j}\right|$, leaving us with $-q$. This will be true if we have a 'cycle' of $\dot{G}_{B}$ 's. That is, a product of $\dot{G}_{B}$ 's with indices arranged as

$$
\dot{G}_{B}\left(\nu_{i_{1} i_{2}}\right) \dot{G}_{B}\left(\nu_{i_{2} i_{3}}\right) \ldots \dot{G}_{B}\left(\nu_{i_{n-1} i_{n}}\right) \dot{G}_{B}\left(\nu_{i_{n} i_{1}}\right) \text {. }
$$

with $\nu_{i_{1}}<\nu_{i_{2}}<\ldots<\nu_{i_{n-1}}<\nu_{i_{n}}$. Such a cycle with more than two $\dot{G}_{B}$ 's will give a contribution of 1 in the limit; a cycle with exactly two $\dot{G}_{B}$ 's will contribute 2 (since both $\dot{G}_{B}$ can contribute a $q$ and the 'cycle' criterion will be fulfilled).

Again, once we have cancelled the $q^{-1}$ the remaining $\dot{G}_{B}$ 's can be expanded as (4.27). Note that if more than one cycle can be identified in any term then each can contribute a $q$; the term produces a sum over the contributions from each cycle. (Note also that they must only contribute one at a time - this is in contrast to cycle contributions from other particles, as we will see later.) "

Once we have dealt with the dominant $q^{-1}$ we can consider the $\tau$ and $\nu_{i}$ integrations. The first step is to change to the new variables $x_{i} \equiv \nu_{i} / \tau$. In these variables (II.2) and (4.27) become

$$
\begin{aligned}
\dot{G}_{B}\left(x_{i j}\right) & \sim-\frac{1}{2} \operatorname{sign}\left(x_{i j}\right)+x_{i j} \\
\prod_{i<j} \exp \left(\alpha^{\prime} G_{B}\left(\nu_{i j}\right) k_{i} \cdot k_{j}\right) & \sim \prod_{i<j} \exp \left(\alpha^{\prime} \sum_{l<m}^{n_{\ell}} P_{i_{l}} \cdot P_{i_{m}} \tau\left(x_{i_{l} i_{m}}^{2}-\left|x_{i_{i_{m}}}\right|\right)\right)
\end{aligned}
$$

where the sum only includes those legs remaining after pinches have been carried out.

The only $\tau$ contribution is now the exponential plus the explicit factors in (4.20). This contribution is independent of the $\dot{G}_{B}$ content, so we can carry out the integration now rather than include it explicitly in the rules. The resulting integration is simply

$$
\int d \tau \tau^{n_{l}-1} \tau^{-2+\epsilon / 2} \exp \left(\alpha^{\prime} \sum_{l<m}^{n_{\ell}} P_{i_{l}} \cdot P_{i_{m}} \tau\left(x_{i_{l} i_{m}}^{2}-\left|x_{i_{i} i_{m}}\right|\right)\right)
$$


Note that the $\tau^{n_{l}-1}$ term here comes from $\prod d \nu_{i}=\prod d x_{i} \tau^{n_{l}-1}$. Equ. (4.33) is recognisable as (4.11) with $K=\alpha^{\prime} \sum_{l<m}^{n_{\ell}} P_{i_{l}} \cdot P_{i_{m}}\left(x_{i_{l} i_{m}}^{2}-\left|x_{i_{l} i_{m}}\right|\right)$, so we get the answer

$$
\frac{1}{\left(\alpha^{\prime}\right)^{n_{l}-2+\epsilon / 2}} \frac{\Gamma\left(n_{l}-2+\epsilon / 2\right)}{\left[\sum_{l<m}^{n_{\ell}} P_{i_{l}} \cdot P_{i_{m}}\left|x_{i_{l} i_{m}}\right|\left(1-\left|x_{i_{l} i_{m}}\right|\right)\right]^{n_{l}-2+\epsilon / 2}}
$$

Finally, we integrate over the region $0<x_{i_{1}}<x_{i_{2}}<\cdots<x_{i_{n_{l}-1}}<x_{i_{n_{l}}}=1$. Note that we can relate the $x_{i}$ parameters to conventional Feynman parameters. Setting $x_{i}=\sum_{j=1}^{i} a_{j}$ shows (4.34) to be a typical Feynman denominator with parameters $a_{j}$.

As required, the combination of pinch and loop contributions has given us an overall factor of $(\alpha)^{-n+n_{l}}(\alpha)^{-n_{l}+2}=(\alpha)^{-n+2}$. This is independent of the number of pinches which we carried out, which implies that we must consider all possible pinch combinations to obtain the complete result.

So, we can see the basic plan for the rules: We can take into account the various pinch contributions by considering all the diagrams coming from the different ways of pinching (as in fig. 4.G) - that is, all $\phi^{3}$ one-loop diagrams (except tadpoles and those with the loop on an external leg). For each diagram we begin by looking at the effect of pinches present and then look at the two ways the loop contributes. This produces a Feynman parameter integral which we can easily evaluate. Then a sum over all contributions from diagrams gives the required amplitude.

As we said, this string contains only a spin-one particle in its massless sector, so the analysis above will only lead to rules for pure gluon amplitudes. We may wonder if we can derive rules for other particles 'circulating in the loop'. We find that we can do this with relatively small changes. The simplest case is for scalar particles in the loop. We can find the rules for these by making a small adaption of the string: If we compactify some dimensions before making our 'naive truncation', we will introduce scalars into the spectrum $[82,65]$. We find that $(4.20)$ changes by an overall factor of

$$
1+n_{s} q+\mathcal{O}\left(q^{2}\right)
$$


( $n_{s}$ is the number of scalars). From this we can easily deduce that scalars will have similar rules to gluons, but without cycle contributions.

The bosonic string spectrum does not contain fermionic particles, so we cannot deduce rules for fermions in the loop directly by the above method. We can, however, find such a set of rules indirectly by looking at the superstring rules. The superstring expression has an extra component constructed from fermionic Green functions, $G_{F}^{i j}$. It was noted in ref. [3] that (after removing $\ddot{G}_{B}$ terms via the integration by parts process) the kinematic function found in the superstring rules would vanish if the substitution $G_{F}^{i j} \rightarrow-\dot{G}_{B}^{i j}$ was made. This suggests that all the information we need is in some way encoded in the bosonic component of the superstring, and hence in our bosonic string expression. By looking at the superstring rules, we can deduce rules which extract this information.

\subsection{QCD Rules [14]}

After considering the analysis from string theory, we can simplify the procedure to give a simple set of rules which encode the calculational steps required while discarding the unwanted string theory artifacts. In this way the method can easily be used by those who do not have a prior knowledge of string theory. Here we give these rules in their simplified form. We also detail the differences required for particles other than gluons in the loop.

The starting point for the rules is to identify all relevant diagrams. Recall that we must include diagrams corresponding to all pinch combinations. In practice, this means drawing all 1-loop $\phi^{3}$ diagrams with the correct number of external legs (except 'tadpole' diagrams and those with bubbles on external legs). Since we are considering the colour ordered form of the amplitude, only labellings of the external legs which follow the cyclic ordering of the colour trace need be included. Internal legs are labelled according to the pinch rule at a three point vertex the internal leg is given the label of the most clockwise of the two outer legs. We then derive an expression for each diagram and sum the contributions at the end. 
For each diagram we have the integration

$$
\begin{aligned}
\mathcal{D}=\frac{\Gamma\left(n_{l}-2+\frac{1}{2} \epsilon\right)}{(4 \pi)^{2-\epsilon / 2}} \int_{0}^{1} d x_{i_{n_{\ell}-1}} \int_{0}^{x_{i_{n_{\ell}-1}}} d x_{i_{n_{\ell}-2}} \cdots \int_{0}^{x_{i_{3}}} d x_{i_{2}} \int_{0}^{x_{i_{2}}} d x_{i_{1}} \\
\times \frac{K_{\mathrm{red}}\left(x_{i_{1}}, \ldots, x_{i_{n_{\ell}}}\right)}{\left(\sum_{l<m}^{n_{\ell}} P_{i_{l}} \cdot P_{i_{m}} x_{i_{m} i_{l}}\left(1-x_{i_{m} i_{l}}\right)\right)^{n_{\ell-2+\epsilon}}}
\end{aligned}
$$

where $n_{l}$ is the number of legs attached to the loop.

$K_{\text {red }}$ is the 'reduced kinematic factor'. To obtain the this we begin with the full kinematic expression

$$
\mathcal{K}=\left.\int \prod_{i=1}^{n} d x_{i} \prod_{i<j}^{n} \exp \left[k_{i} \cdot k_{j} G_{B}^{i j} \cdot\left(k_{j} \cdot \epsilon_{i}-k_{i} \cdot \epsilon_{j}\right) \dot{G}_{B}^{i j}-\epsilon_{i} \cdot \epsilon_{j} \ddot{G}_{B}^{i j}\right]\right|_{\text {multi-linear }}
$$

which will be recognised as a simplified form of the numerator in the string integrand, with $G_{B}^{i j}=G_{B}\left(\nu_{i j}\right)$.

While we know from earlier sections that $G_{B}$ and its derivatives are string theory Green functions, in these simplified rules we can regard them simply as functions of Feynman parameters. It is useful to note that $G_{B}$ and $\ddot{G}_{B}$ are symmetric, while $\dot{G}_{B}$ is antisymmetric.

As in the derivation, we begin by integrating by parts to remove the $\ddot{G}_{B}$ 's from the expression (in the same way as our earlier example, (4.22) and (4.23)). Once this has been done we can drop the $\prod d x_{i} \prod \exp \left[k_{i} \cdot k_{j} G_{B}^{i j}\right]$ term from the expression. (Recall from the derivation that the only explicit contribution from this term was in the denominator resulting from the $\tau$ integration; this has already been accounted for in (4.35).)

So, we are now left with a kinematic expression consisting of a sum of products of 'Feynman parameter functions'. Our next step is to deal with the pinch, or tree, contributions. For any tree attached to the loop we consider each vertex in turn, beginning with those outside and working in. At each vertex we make the substitutions in fig. 4.J.

Once we have done this for every tree in the diagram being considered, we move on to the loop rules. We saw in the derivation, that there are two types of loop contribution 


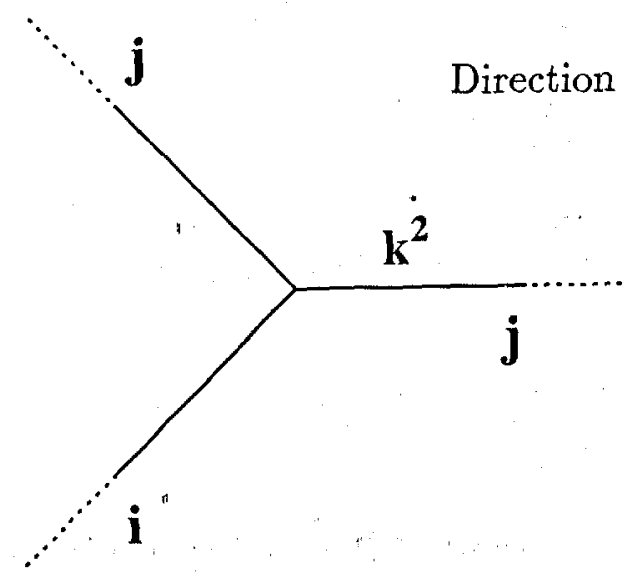

$$
\begin{aligned}
\left(\dot{G}_{B}^{i j}\right)^{n} & \rightarrow-\frac{\delta_{n, 1}}{k^{2}}=-\frac{\delta_{n, 1}}{2 k_{i} \cdot k_{j}} \\
i & \rightarrow j \quad \text { in remaining terms }
\end{aligned}
$$

Figure 4.J: The Tree Rules. Note that leg i appears before leg $\mathrm{j}$ in the (clockwise) cyclic ordering.

we must consider. For the first, we multiply the kinematic expression by $2\left(1-\frac{1}{2} \delta_{R} \epsilon\right)$ and make the substitution

$$
\dot{G}_{B} \rightarrow-\frac{1}{2} \operatorname{sign}\left(x_{i j}\right)+x_{i j} \quad \cdot \quad \cdot
$$

The second contribution comes when we have terms containing 'cycles' of Green functions. As we discussed in the last section these are products of Green functions with indices:

$$
\dot{G}_{B}^{i_{1} i_{2}} \dot{G}_{B}^{i_{2} i_{3}} \cdots \dot{G}_{B}^{i_{n-1} i_{n}} \dot{G}_{B}^{i_{n} i_{1}}
$$

We must only consider cycles which follow the ordering of the legs. There are two possible contributions, dependent on the length of the cycle. For a cycle containing two $\dot{G}_{B}$ 's we make the substitution

$$
\dot{G}_{B}^{i_{1} i_{2}} \dot{G}_{B}^{i_{2} i_{1}} \rightarrow 2
$$

for one with more than two we substitute

$$
\dot{G}_{B}^{i_{1} i_{2}} \dot{G}_{B}^{i_{2} i_{3}} \ldots \dot{G}_{B}^{i_{n-1} i_{n}} \dot{G}_{B}^{i_{n} i_{1}} \rightarrow 1 \quad(n>2)
$$

We must consider the cycles contributions in each term one at a time. After a cycle substitution has been made we substitute for all other $\dot{G}_{B}$ 's as in (4.37). 
Let us consider a typical term as an example of the loop rules:

$$
\left(\dot{G}_{B}^{12}\right)^{2} \dot{G}_{B}^{34} \dot{G}_{B}^{45} \dot{G}_{B}^{53}
$$

with the legs ordered as $x_{1} \leq x_{2} \leq x_{3} \leq x_{4} \leq x_{5}$. The non-cycle contribution will be

$$
2\left(1-\frac{1}{2} \delta_{R} \epsilon\right)\left(\frac{1}{2}+x_{12}\right)^{2}\left(\frac{1}{2}+x_{34}\right)\left(\frac{1}{2}+x_{45}\right)\left(-\frac{1}{2}+x_{53}\right)
$$

We then have two cycles contributions

$$
\left(\dot{G}_{B}^{12}\right)^{2}=-\dot{G}_{B}^{12} \dot{G}_{B}^{21} \rightarrow-2
$$

and

$$
\dot{G}_{B}^{34} \dot{G}_{B}^{45} \dot{G}_{B}^{53} \rightarrow 1
$$

giving a total loop contribution of

$$
\begin{aligned}
2\left(1-\frac{1}{2} \delta_{R} \epsilon\right)\left(\frac{1}{2}+x_{12}\right)^{2}\left(\frac{1}{2}+x_{34}\right)\left(\frac{1}{2}+x_{45}\right)\left(-\frac{1}{2}+x_{53}\right) \\
-2 \times\left(\frac{1}{2}+x_{34}\right)\left(\frac{1}{2}+x_{45}\right)\left(-\frac{1}{2}+x_{53}\right)+1 \times\left(\frac{1}{2}+x_{12}\right)^{2}
\end{aligned}
$$

Having carried out all the rules and obtained an expression for $K_{\text {red }}$, we can insert this into (4.35) and integrate to obtain the contribution from the diagram being considered. (We can, if we wish, make the substitution $x_{i}=\sum_{j=1}^{i} a_{j}$ to obtain a Feynman parameter integration.) Once we have contributions from all diagrams we simply sum to get an expression for the full amplitude.

The loop rules above give the contribution for a complete gluon (with two degrees of freedom). In general, it will be useful to express the rules for one gluon degree of freedom. If we choose the dimensional regularisation scheme where $\delta_{R}=0$ then we simply omit the overall factor multiplying the non-cycle terms and the cycle substitutions become

$$
\dot{G}_{B}^{i_{1} i_{2}} \dot{G}_{B}^{i_{2} i_{1}} \rightarrow 1
$$


and

$$
\dot{G}_{B}^{i_{1} i_{2}} \dot{G}_{B}^{i_{2} i_{3}} \ldots \dot{G}_{B}^{i_{n-1} i_{n}} \dot{G}_{B}^{i_{n} i_{1}} \rightarrow \frac{1}{2} \quad(n>2)
$$

As we mentioned in the last section, we can also find rules for scalars easily. The result is that we follow the above prescription precisely, but omit cycle terms; after dealing with pinches, we merely make the substitution (4.37) and multiply by an overall factor of $N_{s}$, the number of real scalars. For instance, our previous example term (4.41) would give us the scalar loop contribution

$$
N_{s}\left(1-\frac{1}{2} \delta_{R} \epsilon\right)\left(\frac{1}{2}+x_{12}\right)^{2}\left(\frac{1}{2}+x_{34}\right)\left(\frac{1}{2}+x_{45}\right)\left(-\frac{1}{2}+x_{53}\right)
$$

For fermions, we again find that the only change is in the cycle contributions. Unlike the gluon case, where only cycles of a particular ordering are included, we include cycles of all orderings. The actual contribution for each cycle also differs slightly. For fermions, we make the substitution

$$
\dot{G}_{B}^{i_{1} i_{2}} \dot{G}_{B}^{i_{2} i_{3}} \ldots \dot{G}_{B}^{i_{m-1} i_{m}} \dot{G}_{B}^{i_{m} i_{1}} \rightarrow-\left(-\frac{1}{2}\right)^{m} \prod_{k=1}^{m} \operatorname{sign}\left(x_{i_{k} i_{k+1}}\right)
$$

The other difference from the gluon case is that cycles no longer contribute one at a time, So, if a term has more than one cycle, we must include all possible combinations of contributions. For example, consider again the sample term (4.41); in the fermion case this will give a loop contribution

$$
\begin{aligned}
-N_{f}\left(\left(\frac{1}{2}+x_{12}\right)^{2}\left(\frac{1}{2}+x_{34}\right)\left(\frac{1}{2}+x_{45}\right)\left(-\frac{1}{2}+x_{53}\right)\right. \\
\left.-\frac{1}{4} \times\left(\frac{1}{2}+x_{34}\right)\left(\frac{1}{2}+x_{45}\right)\left(-\frac{1}{2}+x_{53}\right)+\frac{1}{8} \times\left(\frac{1}{2}+x_{12}\right)^{2}+\frac{1}{32}\right)
\end{aligned}
$$

We have also multiplied by an over all factor of minus the number of fermionic degrees of freedom, $-N_{f}$.

It is useful to note at this point that the only momenta which appear in these rules are the on-shell external momenta. We can therefore make full use of the spinor-helicity technique 
described in appendix III to simplify (4.36) at the start of the calculation. An astute choice of reference momenta will significantly reduce the number of terms which we must consider from that expression. This allows significant simplifications over Feynman diagrams, where off-shell loop momenta occur explicitly and restrict the use of spinor-helicity until the end.

\subsection{Sample Calculation [15]}

As an example of this method, let us consider the one-loop four gluon helicity amplitude $A^{1-\text { loop }}\left(1^{-}, 2^{+}, 3^{+}, 4^{+}\right)$. As specified in the rules, we begin the calculation by identifying relevant diagrams. In this case we consider those in fig. 4.K. They are the diagrams with no pinches - (a); with one pinch - (b), (c), (d) and (e); and with two pinches - (f) and (g).

Before considering the kinematic expression, let us try to simplify things by using spinor helicity relations. A useful choice of reference momenta for this amplitude is ( $\left.k_{4}, k_{1}, k_{1}, k_{1}\right)$ (by which we mean, the first particle has reference momentum $k_{4}$, the second $k_{1}$, etc.). We can see immediately that this allows us to identify the following as trivial

$$
\begin{array}{ll}
\epsilon_{i} \cdot \epsilon_{j}=0 & \forall i, j \\
k_{1} \cdot \epsilon_{j}=0 & \forall j \\
k_{4} \cdot \epsilon_{1}=0, &
\end{array}
$$

and, using (4.50) together with momentum conservation, to equate

$$
\begin{array}{ll}
k_{2} \cdot \epsilon_{1}=-k_{3} \cdot \epsilon_{1} & k_{3} \cdot \epsilon_{2}=-k_{4} \cdot \epsilon_{2} \\
k_{2} \cdot \epsilon_{3}=-k_{4} \cdot \epsilon_{3} & k_{2} \cdot \epsilon_{4}=-k_{3} \cdot \epsilon_{4} .
\end{array}
$$

With these simplifications (4.36), with $n=4$, becomes

$$
\begin{aligned}
\mathcal{K}=\left.\int \prod_{i=1}^{4} d x_{i} \prod_{i<j}^{4} \exp \left[k_{i} \cdot k_{j} G_{B}^{i j}-\left(k_{j} \cdot \epsilon_{i}-k_{i} \cdot \epsilon_{j}\right) \dot{G}_{B}^{i j}\right]\right|_{\text {multilinear }} \\
=\int \prod_{i=1}^{4} d x_{i} \prod_{i<j}^{4} \exp \left[k_{i} \cdot k_{j} G_{B}^{i j}\right]\left(k_{2} \cdot \epsilon_{1}\left(\dot{G}_{B}^{12}-\dot{G}_{B}^{13}\right)\right. \\
\left.\quad \times k_{3} \cdot \epsilon_{2}\left(\dot{G}_{B}^{23}-\dot{G}_{B}^{24}\right) k_{2} \cdot \epsilon_{3}\left(\dot{G}_{B}^{23}+\dot{G}_{B}^{34}\right) k_{2} \cdot \epsilon_{4}\left(\dot{G}_{B}^{24}-\dot{G}_{B}^{34}\right)\right)
\end{aligned}
$$



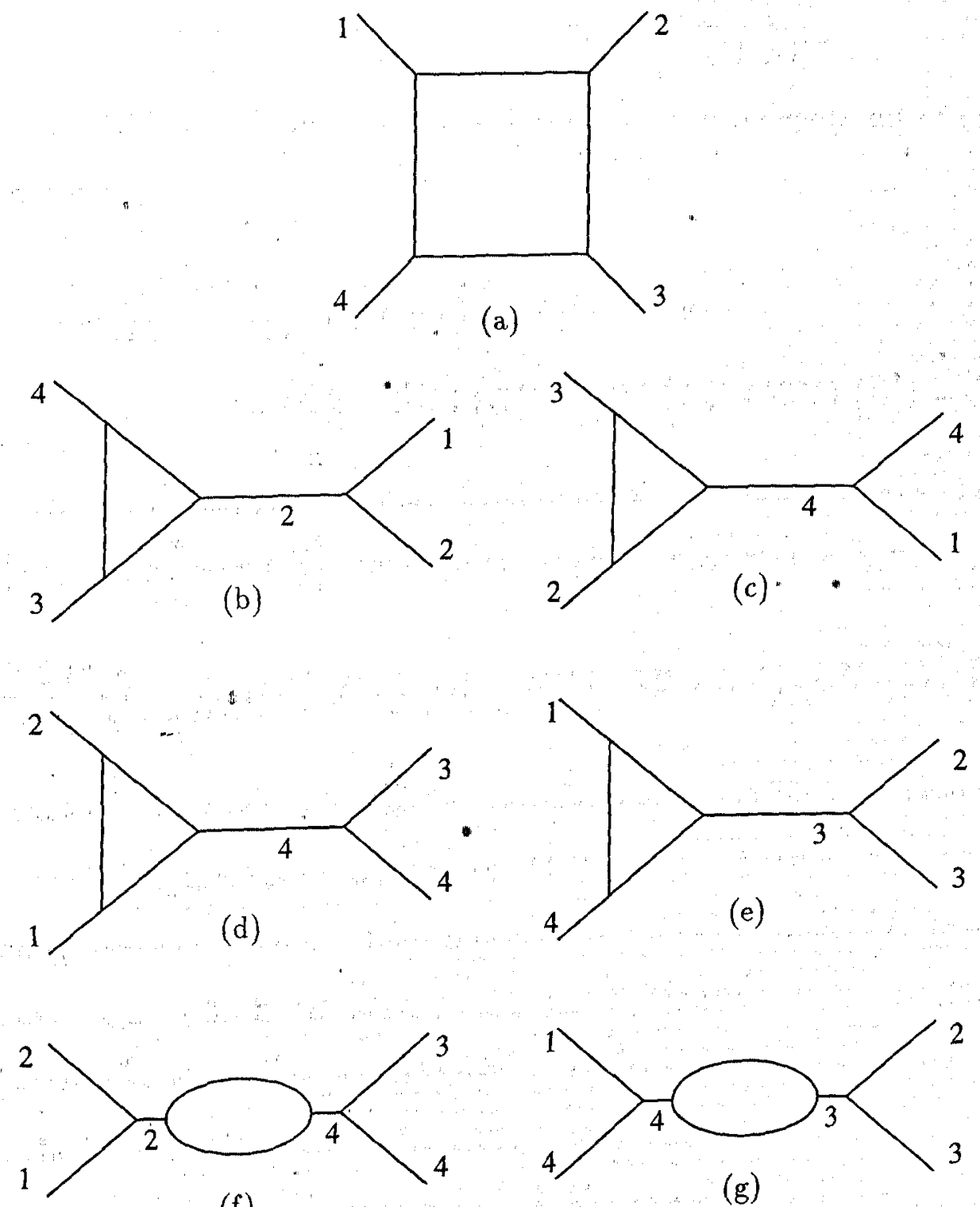

(f)

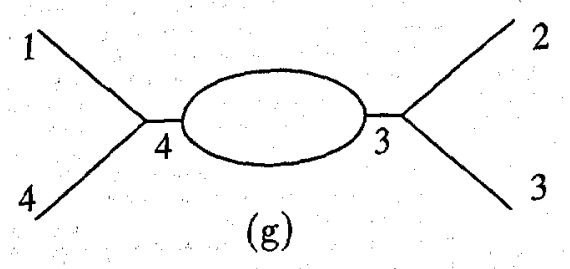

Figure 4.K: Relevant diagrams for the $A^{1-\operatorname{loop}}\left(1^{-}, 2^{+}, 3^{+}, 4^{+}\right)$ gluon calculation. 
Using the spinor helicity expressions to express the dot products in terms of spinor innerproducts,

$$
\begin{array}{ll}
k_{2} \cdot \epsilon_{1}=\frac{[42]\langle 21\rangle}{\sqrt{2}[14]} & k_{3} \cdot \epsilon_{2}=\frac{\langle 13\rangle[32]}{\sqrt{2}\langle 12\rangle} \\
k_{2} \cdot \epsilon_{3}=\frac{\langle 12\rangle[23]}{\sqrt{2}\langle 13\rangle} & k_{2} \cdot \epsilon_{4}=\frac{\langle 12\rangle[24]}{\sqrt{2}\langle 14\rangle}
\end{array}
$$

we can rewrite (4.52) in a form which is both compact and independent of our choice of reference momenta,

$$
\begin{aligned}
\mathcal{K}=\frac{1}{4} \frac{[24]^{2} t s^{2}}{[12]\langle 23\rangle\langle 34\rangle[41]} \int \prod_{i=1}^{4} d x_{i} \prod_{i<j}^{4} \exp \left[k_{i} \cdot k_{j} G_{B}^{i j}\right] \\
\times\left(\dot{G}_{B}^{12}-\dot{G}_{B}^{13}\right)\left(\dot{G}_{B}^{23}-\dot{G}_{B}^{24}\right)\left(\dot{G}_{B}^{23}+\dot{G}_{B}^{34}\right)\left(\dot{G}_{B}^{24}-\dot{G}_{B}^{34}\right)
\end{aligned}
$$

We have no need to integrate by parts here, so we can drop the integral and exponential terms, $\int \prod d x_{i} \prod \exp \left[k_{i} \cdot k_{j} G_{B}^{i j}\right]$, immediately to give the kinematic factor

$$
\frac{1}{4} \frac{[24]^{2} t s^{2}}{[12]\langle 23\rangle\langle 34\rangle[41]}\left(\dot{G}_{B}^{12}-\dot{G}_{B}^{13}\right)\left(\dot{G}_{B}^{23}-\dot{G}_{B}^{24}\right)\left(\dot{G}_{B}^{23}+\dot{G}_{B}^{34}\right)\left(\dot{G}_{B}^{24}-\dot{G}_{B}^{34}\right)
$$

Looking at the effect of pinch rules for the various diagrams, we can instantly see that diagram (c) vanishes: there is no $\dot{G}_{B}^{14}$ term in (4.53), so the pinch contribution vanishes. Also (e) and (g) vanish: when a $2-3$ pinch occurs $\left(\dot{G}_{B}^{12}-\dot{G}_{B}^{13}\right)$ becomes $\left(\dot{G}_{B}^{13}-\dot{G}_{B}^{13}\right)=0$, since we must set $2 \rightarrow 3$. In the same way, (d) and (f) have zero contribution because $\left(\dot{G}_{B}^{23}-\dot{G}_{B}^{24}\right)$ vanishes when there is a 3-4 pinch. These can all be dismissed before the loop rules are applied and so give no contribution, scalar or cycle.

So, we are left with (a) and (b). Let us look first at the scalar contribution to these, beginning with (a). This has no pinches, so we go straight to the loop rules. The loop substitutions give us

$$
\begin{aligned}
K_{\mathrm{red}}^{(a)} & =\frac{1}{4} \frac{[24]^{2} t s^{2}}{[12]\langle 23\rangle\langle 34\rangle[41]}\left(x_{12}-x_{13}\right)\left(x_{23}-x_{24}\right)\left(1+x_{23}+x_{34}\right)\left(x_{24}-x_{34}\right) \\
& =\frac{1}{4} \frac{[24]^{2} t s^{2}}{[12]\langle 23\rangle\langle 34\rangle[41]}\left(x_{2}-x_{3}\right)\left(x_{3}-x_{4}\right)\left(1+x_{2}-x_{4}\right)\left(x_{2}-x_{3}\right)
\end{aligned}
$$


Substituting this into (4.35) gives us

$$
\begin{aligned}
\mathcal{D}_{s}^{(a)}=\frac{\Gamma\left(n_{l}-2+\frac{1}{2} \epsilon\right)}{(4 \pi)^{2-\epsilon / 2}} & \frac{1}{4} \frac{[24]^{2} t^{2} s}{[12]\langle 23\rangle\langle 34\rangle[41]} \\
& \times j_{0}^{1} d x_{3} \int_{0}^{x_{3}} d x_{2} \int_{0}^{x_{2}} d x_{1} \frac{\left(x_{2}-x_{3}\right)^{2}\left(x_{3}-1\right) x_{2}}{D^{2+\epsilon / 2}}
\end{aligned}
$$

Where $x_{4}$ has been set to 1 and

$$
\begin{aligned}
D & =\sum_{i>j}^{4} k_{i} \cdot k_{j} x_{i j}\left(1-x_{i j}\right) \\
& =\left(-s x_{1}\left(x_{3}-x_{2}\right)-t\left(x_{2}-x_{1}\right)\left(1-x_{3}\right)\right)
\end{aligned}
$$

We can convert (4.55) into a Feynman parameter integral with the substitution $x_{i}=\sum_{j=1}^{i} a_{j}$ :

$$
\begin{aligned}
& \mathcal{D}_{s}^{(a)}=\frac{\Gamma\left(n_{l}-2+\frac{1}{2} \epsilon\right)}{(4 \pi)^{2-\epsilon / 2}} \frac{1}{4} \frac{[24]^{2} \dot{t s}^{2}}{[12]\langle 23\rangle\langle 34\rangle[41]} \\
& \times \int_{0}^{1} \prod_{i=1}^{4} d a_{i} \delta\left(1-\sum_{i=1}^{4} a_{i}\right) \frac{a_{3}^{2} a_{4}\left(a_{1}+a_{2}\right)}{\left(-s a_{1} a_{3}-t a_{2} a_{4}\right)^{2+\epsilon / 2}}
\end{aligned}
$$

This can then be integrate (for example, with an integral table constructed by the methods outlined in ref. [83]) to give:

$$
\mathcal{D}_{s}^{(a)}=\frac{\Gamma\left(n_{l}-2+\frac{1}{2} \epsilon\right)}{(4 \pi)^{2-\epsilon / 2}} \frac{1}{4} \frac{[24]^{2} t s^{2}}{[12]\langle 23\rangle\langle 34\rangle[41]} \times \frac{1}{6 s t}
$$

For (b), we must first apply the pinch rules. So, we substitute

$$
\left(\dot{G}_{B}^{12}\right)^{n}=\frac{\delta_{n, 1}}{2 k_{1} \cdot k_{2}}=\frac{\delta_{n, 1}}{s}
$$

and change the indices $1 \rightarrow 2$ in remaining terms. This gives

$$
\begin{aligned}
& \frac{1}{4} \frac{[24]^{2} t s^{2}}{[12]\langle 23\rangle\langle 34\rangle[41]} \frac{1}{s}\left(\dot{G}_{B}^{23}-\dot{G}_{B}^{24}\right)\left(\dot{G}_{B}^{23}+\dot{G}_{B}^{34}\right)\left(\dot{G}_{B}^{24}-\dot{G}_{B}^{34}\right) \\
& =\frac{1}{4 s} \frac{[24]^{2} t s^{2}}{[12]\langle 23\rangle\langle 34\rangle[41]}\left(x_{3}-1\right)\left(x_{2}-2\right)\left(x_{2}-x_{3}\right)
\end{aligned}
$$

Then, similar to (a), we get

$$
\begin{aligned}
\mathcal{D}_{s}^{(b)}=\frac{\Gamma\left(n_{l}-2+\frac{1}{2} \epsilon\right)}{(4 \pi)^{2-\epsilon / 2}} \frac{1}{4} \frac{[24]^{2} t s^{2}}{[12]\langle 23\rangle\langle 34\rangle[41]} & \\
& \times \int_{0}^{1} d x_{3} \int_{0}^{x_{3}} d x_{2} \frac{\left(x_{2}-x_{3}\right)\left(x_{3}-1\right) x_{2}}{D^{1+\epsilon / 2}}
\end{aligned}
$$


where $x_{4}$ has been set to 1 and

$$
D=\sum_{i>j=2}^{4} K_{i} \cdot K_{j} x_{i j}\left(1-x_{i j}\right)
$$

$K_{i}$ is the momentum in the leg attached to the loop with label $i$, so this evaluates to

$$
D=s x_{2}\left(x_{2}-x_{3}\right)
$$

and the integral, in the Feynman parameter form, is

$$
\begin{aligned}
\mathcal{D}_{s}^{(b)}=\frac{\Gamma\left(n_{l}-2+\frac{1}{2} \epsilon\right)}{(4 \pi)^{2-\epsilon / 2}} \frac{1}{4 s} \frac{[24]^{2} t s^{2}}{[12]\langle 23\rangle\langle 34\rangle[41]} & \\
& \times \int_{0}^{1} \prod_{i=2}^{4} d a_{i} \delta\left(1-\sum_{i=2}^{4} a_{i}\right) \frac{a_{3} a_{4}\left(a_{2}-2\right)}{\left(-s a_{2} a_{3}\right)^{1+\epsilon / 2}}
\end{aligned}
$$

Which gives

$$
\mathcal{D}_{s}^{(b)}=\frac{\Gamma\left(n_{l}-2+\frac{1}{2} \epsilon\right)}{(4 \pi)^{2-\epsilon / 2}} \frac{1}{4} \frac{[24]^{2} t s^{2}}{[12]\langle 23\rangle\langle 34\rangle[41]} \times \frac{1}{6 s^{2}}
$$

Combining (4.58) and (4.65) we get the result

$$
\mathcal{D}_{\dot{s}}=\frac{\Gamma\left(n_{l}-2+\frac{1}{2} \epsilon\right)}{(4 \pi)^{2-\epsilon / 2}} \frac{u}{24} \frac{[24]^{2} t s^{2}}{[12]\langle 23\rangle\langle 34\rangle[41]}
$$

Now let us look at the extra cycle contributions for the gluon. If we expand (4.53),

$$
\begin{array}{r}
\frac{1}{32} \frac{[24]^{2} t s^{2}}{[12]\langle 23\rangle\langle 34\rangle[41]}\left(\dot{G}_{B}^{12}-\dot{G}_{B}^{13}\right)\left(\left(\dot{G}_{B}^{23}\right)^{2} \dot{G}_{B}^{24}-\left(\dot{G}_{B}^{24}\right)^{2} \dot{G}_{B}^{23}+\dot{G}_{B}^{24}\left(\dot{G}_{B}^{34}\right)^{2}\right. \\
\left.-\left(\dot{G}_{B}^{24}\right)^{2} \dot{G}_{B}^{34}-\left(\dot{G}_{B}^{23}\right)^{2} \dot{G}_{B}^{34}-\dot{G}_{B}^{23}\left(\dot{G}_{B}^{34}\right)^{2}+\dot{G}_{B}^{23} \dot{G}_{B}^{34} \dot{G}_{B}^{24}+\dot{G}_{B}^{24} \dot{G}_{B}^{23} \dot{G}_{B}^{34}\right)
\end{array}
$$

we can identify cycles in every term - two-cycles in the first six here and three-cycles in the remaining two. Making the cycle substitutions, this becomes

$$
\begin{aligned}
& \frac{1}{32} \frac{[24]^{2} t s^{2}}{[12]\langle 23\rangle\langle 34\rangle[41]}\left(\dot{G}_{B}^{12}-\dot{G}_{B}^{13}\right) \\
& \times\left(-2\left(\dot{G}_{B}^{24}-\dot{G}_{B}^{23}+\dot{G}_{B}^{24}-\dot{G}_{B}^{34}-\dot{G}_{B}^{34}-\dot{G}_{B}^{23}\right)-1-1\right)
\end{aligned}
$$

We then make the loop substitutions and obtain

$$
\frac{1}{32} \frac{[24]^{2} t s^{2}}{[12]\langle 23\rangle\langle 34\rangle[41]}\left(x_{2}-x_{3}\right)\left(-2\left(2\left(\frac{1}{2}+x_{24}\right)-2\left(\frac{1}{2}+x_{23}\right)-2\left(\frac{1}{2}+x_{34}\right)\right)-2\right),
$$

which is clearly zero. 
The gluon cycles for (b) also vanish since it has essentially the same sum of contributions. Similarly, there will be no fermion cycle contribution for any of the diagrams. So, the total loop contribution is the same for every particle content (up to a numerical constant):

$$
\mathcal{D}=N_{s} \frac{\Gamma\left(n_{l}-2+\frac{1}{2} \epsilon\right)}{(4 \pi)^{2-\epsilon / 2}} \frac{u}{3} \frac{[24]^{2} t s^{2}}{[12]\langle 23\rangle\langle 34\rangle[41]}
$$

Where $N_{s}=N_{b}-N_{f}$, the number of bosonic minus the number of fermionic degrees of freedom.

So, we have shown how we can use an open bosonic string to simplify QCD amplitude calculations. By looking at the infinite tension limit of such a string we can construct a set of rules for finding gluon scattering amplitudes. These rules prove to be significantly more efficient than conventional techniques, such as Feynman diagrams, as we demonstrated with a sample calculation. In the next chapter we will look at the ways in which we can use this type of analysis to help us in PQG. 
Remember that what pulls the strings is the force hidden within; there lies the power to persuade...

Marcus Aurelius Antoninus

\section{String-Based Rules II: Gravity.}

In the previous chapter we used open bosonic strings to construct rules for the one-loop amplitudes with external spin one particles and particles of spin one, one half and zero in the loop. We now turn to the case of gravity; for this we need a system which includes particles of spins up to two. This entails a switch from using open bosonic strings to closed, since the former only includes particles of $\operatorname{spin} \leq 1$. As might be expected, this leads to us a set of rules which have a similar structure to the QCD rules outlined in chapter 4, but for which there are also significant differences. In the next section we will outline the derivation in the closed string case. We will pay particular attention to ways in which it differs from the open string analysis. Once we have considered the essential features of the derivation, we will give the complete set of rules for one-loop calculations containing external gravitons and present the results which have been obtained using these.

Before we look at the derivation let us identify the differences we expect to see. We know that one loop closed ștring amplitudes can be described as a torus with vertex operators on the external surface, rather than the annulus with vertex operators on the boundaries in the open string case. Since the operators are now located on a two-dimensional surface we describe their locations by complex variables instead of real ones. The closed nature also leads to different boundary conditions which generate 'left'- and 'right'-moving components 'in the string theory and 'left'- and 'right'- moving variables. As a result, we will find that our starting amplitude expression is the product of two copies of the open string expression, the left- and right-moving components, plus an interaction term. So, we expect that our 
kinematic term will resemble that in the QCD rules squared plus terms which mix the left and right moving variables. The pinch rules will require a contribution from both components; the loop rules look very similar to the open rules applied to each component independently. We also find that we must express our kinematic factor in a different way; the mixing terms introduce extra powers of $\tau^{-1}$ which will adjust our use of (4.11). In order to take this into account we find it is necessary to postpone the $\tau$ integration and include it as an explicit feature of the rules.

Since our calculation is on a torus the vertex operators are not restricted to lie in a certain order. This will be reflected in the rules by a sum over all orderings of external legs.

\subsection{Derivation of Gravity Rules from Closed Strings}

In this section we look at the derivation of the gravity rules from closed strings. As there are many similarities to the $\mathrm{QCD} /$ open string case, we will concentrate on the differences outlined above.

In this case the particle structure is slightly more complex than that seen in the last chapter; the massless sector of the closed bosonic string contains a symmetric tensor - the graviton - as expected, but it also includes an antisymmetric tensor and a scalar. In four dimensions, the antisymmetric tensor has only one independent component and so looks like a second scalar. So, initially we will be constructing rules which will be valid for a graviton plus two (real) scalars in the loop.

In order to carry out a one-loop closed string amplitude we map the torus onto the complex plane. We then integrate over all positions of vertex operators. The vertex operator for the graviton is

$$
V \sim \kappa: \epsilon_{\mu \nu} \frac{\partial X^{\mu}}{\partial \nu} \frac{\partial X^{\nu}}{\partial \bar{\nu}} e^{i k \cdot X}
$$

We can factorise $\epsilon_{\mu \nu}=\epsilon_{\mu} \bar{\epsilon}_{\nu}[84]$ and so rewrite this as

$$
V \sim \kappa:\left.\exp i\left(k \cdot X+\epsilon \cdot \partial_{\nu} X+\bar{\epsilon} \cdot \partial_{\bar{\nu}} X\right)\right|_{\text {linear }}:
$$


The 'linear' condition implies that we keep terms linear in $\epsilon$ and $\bar{\epsilon}$. We then insert this into (4.6). Again, let us begin by looking at a generic term. We find that we have two copies of the closed string result, one dependent on $\nu_{i j}$ and the other on $\bar{\nu}_{i j}$, plus an term which mixes the left- and right-movers

$$
\begin{array}{r}
\exp \left(k_{i} \cdot k_{j} G_{B}\left(\nu_{i j}\right)+\left(k_{i} \cdot \epsilon_{j}-k_{j} \cdot \epsilon_{i}\right) \dot{G}_{B}\left(\nu_{i j}\right)-\epsilon_{i} \cdot \epsilon_{j} \ddot{G}_{B}\left(\nu_{i j}\right)\right. \\
\left.+\left(k_{i} \cdot \epsilon_{j}-k_{j} \cdot \epsilon_{i}\right) \dot{\bar{G}}_{B}\left(\nu_{i j}\right)-\epsilon_{i} \cdot \epsilon_{j} \ddot{\bar{G}}_{B}\left(\nu_{i j}\right)\right) \\
\quad \times\left.\exp \left(-\left(\epsilon_{i} \cdot \bar{\epsilon}_{j}+\epsilon_{j} \cdot \bar{\epsilon}_{i}\right) H_{B}^{i j}\right)\right|_{\text {multi-linear }}
\end{array}
$$

('Multi-linear' is the generalisation of 'linear' above.) $G_{B}$ is defined as before as

$$
\left\langle X_{\mu} X_{\nu}\right\rangle=\delta_{\mu \nu} G_{B}\left(\nu_{i}-\nu_{j}\right)
$$

and

$$
\begin{aligned}
\dot{G}_{B}(\nu)=\frac{1}{2} \partial_{\nu} G_{B}(\nu) & \dot{\bar{G}}_{B}(\bar{\nu})=\frac{1}{2} \partial_{\bar{\nu}} G_{B}(\nu) \\
\ddot{G}_{B}(\nu)=\frac{1}{4} \partial_{\nu}^{2} G_{B}(\nu) & \ddot{\bar{G}}_{B}(\bar{\nu})=\frac{1}{4} \partial_{\bar{\nu}}^{2} G_{B}(\nu)
\end{aligned}
$$

The mixing term comes from the part of the expansion which looks like

(see appendix II).

$$
\begin{aligned}
\epsilon_{i}^{\mu} \bar{\epsilon}_{j}^{\nu} & \left\langle\partial_{\nu_{i}} X_{\mu} \partial_{\bar{\nu}_{j}} X_{\nu}\right\rangle+\bar{\epsilon}_{i}^{\mu} \epsilon_{j}^{\nu}\left\langle\partial_{\bar{\nu}_{i}} X_{\mu} \partial_{\nu_{j}} X_{\nu}\right\rangle \\
& =\left(\epsilon_{i}^{\mu} \bar{\epsilon}_{j}^{\nu} \ddot{\partial}_{\nu_{i}} \partial_{\bar{\nu}_{j_{o}}}+\bar{\epsilon}_{i}^{\mu} \epsilon_{j}^{\nu} \partial_{\bar{\nu}_{i}} \partial_{\nu_{j}}\right) G_{B}\left(\nu_{i j}\right) \\
& =-\left(\epsilon_{i}^{\mu} \bar{\epsilon}_{j}^{\nu}+\bar{\epsilon}_{i}^{\mu} \epsilon_{j}^{\nu}\right) H_{B}^{i j}
\end{aligned}
$$

So, we eventually end up with an expression for the amplitude ${ }^{\dagger}$

$$
\begin{aligned}
A_{n}= & i \frac{(4 \pi)^{\epsilon / 2}}{16 \pi^{2}}(\sqrt{2})^{n} \kappa^{n}\left(\alpha^{\prime}\right)^{-2} \\
& \times \int_{0}^{\infty} d^{2} \tau(\Im \tau)^{-2+\epsilon / 2} \int \prod_{i=1}^{n-1} d^{2} \nu_{i} \theta\left(\left|\nu_{i}\right|-\left|\nu_{i+1}\right|\right) \bar{q}^{-1} q^{-1}[f(q) f(\bar{q})]^{-2\left(1-\delta_{R} \epsilon / 2\right)} \\
& \times \prod_{i<j}^{n} \exp \left(\alpha^{\prime} k_{i} \cdot k_{j} G_{B}\left(\nu_{i j}\right)+\sqrt{\alpha^{\prime}}\left(k_{i} \cdot \epsilon_{j}-k_{j} \cdot \epsilon_{i}\right) \dot{G}_{B}\left(\nu_{i j}\right)-\epsilon_{i} \cdot \dot{\epsilon}_{j} \ddot{G}_{B}\left(\nu_{i j}\right)\right. \\
& \left.+\sqrt{\alpha^{\prime}}\left(k_{i} \cdot \bar{\epsilon}_{j}-k_{j} \cdot \bar{\epsilon}_{i}\right) \dot{\bar{G}}_{B}\left(\bar{\nu}_{i j}\right)-\bar{\epsilon}_{i} \cdot \bar{\epsilon}_{j} \ddot{G}_{B}\left(\bar{\nu}_{i j}\right)-\left(\epsilon_{i} \cdot \bar{\epsilon}_{j}+\bar{\epsilon}_{i} \cdot \epsilon_{j}\right) H_{B}^{i j}\right)\left.\right|_{\text {multi-l-l}}
\end{aligned}
$$

$\dagger$ Recall, from chapter 3 , that we have chosen not to write the coupling explicitly as $\mu^{\epsilon} \kappa$ in $4-2 \epsilon$ dimensions. 
$q$ and $\bar{q}$ are defined as $\exp (-2 \tau)$ and $\exp (-2 \bar{\tau})$, respectively. Note that we have an implicit ordering of the $\nu$ 's in the integral: $\left|\nu_{1}\right|<\ldots<\left|\nu_{n}\right|=|\tau|$. This has been imposed to allow us to use Wick's Theorem. Closed string amplitudes do not have any natural ordering of the legs (since they are located on the surface of a torus); we will take this into account in the rules by summing over contributions from all orderings of the external labels.

As with open string case we begin by integrating by parts to remove $\ddot{G}_{B}$ and $\ddot{G}_{B}$ terms. We can do this for the left and right terms in turn, but must be careful to look out for those places where a differentiation with respect to $\left(\begin{array}{c}\text { left } \\ \text { right }\end{array}\right)$ moving variables hits ( $\left.\begin{array}{c}\text { right } \\ \text { left }\end{array}\right)$ moving functions to produce $H_{B}$ 's. We will give an example of this when we give a final version of the rules.

Assuming we have carried out this process, let us look at the effect of the $\alpha^{\prime} \rightarrow 0$ limit. It is easy to check that, when the exponential is expanded to fulfil the 'multi-linear' condition, a term containing $k H_{B}$ 's will have a overall factor of $\left(\dot{\alpha}^{\prime}\right)^{n-k-2}$. As before, we will find 'pinch' and 'loop' contributions which produce sufficient powers of $\left(\alpha^{\prime}\right)^{-1}$ to cancel this. First, let us look at the way the pinches work in this case.

Pinch contributions come from the effect of $\left|\nu_{i}^{\prime}-\nu_{j}\right| \rightarrow 0$. Evidently, this also means that $\left|\bar{\nu}_{i}-\bar{\nu}_{j}\right| \rightarrow 0$, so we expect contributions from both $\dot{G}_{B}^{i j}$ and $\dot{G}_{B}^{i j}$. In the limit we will find integrals of the form

$$
\int d^{2} \nu_{i} \frac{1}{\left(\nu_{i j} \bar{\nu}_{i j}\right)^{-\alpha^{\prime} k_{i} \cdot k_{j} / 2 \pi}} \nu_{i j}^{m} \bar{\nu}_{i j}^{n}
$$

(cf $(4.24))$. The $|\nu|^{-\alpha^{\prime} k_{i} \cdot k_{j} / \pi}$ comes from the $\exp \left(G_{B}^{i j}\right)$ term; each $\dot{G}_{B}^{i j}$ and $\dot{\bar{G}}_{B}^{i j}$ produces a $\nu_{i j}^{-1}$ and $\bar{\nu}_{i j}^{-1}$ respectively. This integral will only give a contribution which survives as $\alpha^{\prime} \rightarrow 0$ if $n=m=1$; if $n \neq m$ then the integral vanishes and if $n=m \neq 1$ then the result will be $1 /\left(\alpha^{\prime}\right.$-integer $)$ which gives a trivial contribution in the limit as $\alpha^{\prime} \rightarrow 0$. For $n=m=1$ (5.8) becomes:

$$
\int d^{2} \nu_{i} \frac{1}{\left(\nu_{i j} \bar{\nu}_{i j}\right)^{1+\alpha^{\prime} k_{i} \cdot k_{j} / 2 \pi}} \longrightarrow-\frac{2 \pi}{\alpha^{\prime} k_{i} \cdot k_{j}} \quad \alpha^{\prime} \rightarrow 0
$$


So, a pinch contribution in the closed string rules will arise when we have exactly one $\dot{G}_{B}^{i j}$ and one $\dot{G}_{B}^{i j}$ in a term. We can compare this to the open string case in which a pinch contribution occured for a term containing exactly one $\dot{G}_{B}^{i j}$. Apart from this difference, the pinches will function in precisely the same way as the previous case: we must substitute $\nu_{i} \rightarrow \nu_{j}$ in all other terms, the number of integrations will be reduced to $n_{l}$ (the number of legs remaining attached to the loop), the exponential will look like (4.26) and we will generate an overall factor of $\left(\alpha^{\prime}\right)^{-n+n_{l}}$.

We now turn to the loop contributions. Here we find that the relevant portion of the $\tau$ integral is that in which $\Im \tau \rightarrow \infty$. This implies that we also restrict the $\nu_{i}$ integrations to the regions where $\left|\Im \nu_{i}\right| \rightarrow \infty$. As with the open string case, we must deal with exponential functions of $\tau$. In this case, we see that (5.7) has factors of both $q^{-1}$ and $\bar{q}^{-1}$. We treat barred and unbarred variables independently, so, up to the point at which we carry out the $\Im \tau$ integration, we can follow the open string analysis for the loop contributions on the left and right movers independently: For each 'half' we must include the gluon cycle and non-cycle contributions that we used before. When applying these loop rules we can treat the $H_{B}$ 's as constants, since they have no relevant contribution. The only subtlety. in this process comes when making the substitution for $\dot{G}_{B}$ and $\dot{\bar{G}}_{B}$ in terms of $x_{i}$ 's. Since both are dependent on $\Im \nu=-\Im \bar{\nu}$, both have the same substitution:

$$
\begin{aligned}
& \dot{G}_{B}\left(x_{i j}\right) \sim-\frac{1}{2} \operatorname{sign}\left(x_{i j}\right)+x_{i j} \\
& \dot{\bar{G}}_{B}\left(x_{i j}\right) \sim-\frac{1}{2} \operatorname{sign}\left(x_{i j}\right)+x_{i j}
\end{aligned}
$$

(There is a relative minus sign ignored here, but this is absorbed into other factors; See appendix II.)

The significant difference comes when we have carried out this analysis and try to integrate over $\Im \tau$. The remaining terms with $\Im \tau$ dependence are

$$
\int d \Im \tau(\Im \tau)^{n_{l}}(\Im \tau)^{-2+\epsilon / 2} \prod_{i<j} \exp \left(\alpha^{\prime} k_{i} \cdot k_{j}\left(x_{i j}^{2}-\left|x_{i j}\right|\right) \Im \tau-\left(\epsilon_{i} \cdot \bar{\epsilon}_{j}+\bar{\epsilon}_{i} \cdot \epsilon_{j}\right) H_{B}^{i j}\right)
$$

Recall that we must impose the 'multi-linear' condition. This implies that, in general, we 
will have a product of $H_{B}$ 's. So, a generic integral will be

$$
\int d \Im \tau(\Im \tau)^{n_{i}}(\Im \tau)^{-2+\epsilon / 2} \prod_{i<j} \exp \left(\alpha^{\prime} k_{i} \cdot k_{j} \Im \tau\left(x_{i j}^{2}-\left|x_{i j}\right|\right)\right) H_{B}^{k_{1} l_{1}} H_{B}^{k_{2} l_{2}} \cdots H_{B}^{k_{l} l_{r}}
$$

We know that in the $|\tau| \rightarrow \infty$ limit $H_{B}^{i j} \sim 1 / \Im \tau$ (appendix I), so this integral becomes

$$
\sim \int d \Im \tau(\Im \tau)^{n_{i}}(\Im \tau)^{-2+\epsilon / 2}(\Im \tau)^{-k} \prod_{i<j} \exp \left(\alpha^{\prime} k_{i} \cdot k_{j} \Im \tau\left(x_{i j}^{2}-\left|x_{i j}\right|\right)\right)
$$

Referring back to (4.33) and (4.34), we can see that the integral will introduce $\left(\alpha^{\prime}\right)^{-n_{l}+2+k}$. This, coupled with the $\left(\alpha^{\prime}\right)^{-n+n_{l}}$ from pinches, will exactly cancel the leading $\left(\alpha^{\prime}\right)^{n-2-k}$ in each term. The number of powers of the Feynman denominator will also depend on the number of $H_{B}$ 's. So, whereas in the open string case we did not have to include the $\tau$ integration in the rules, here it will be necessary to do so. (Note that we could have included the $\tau$ integration in the open string rules and obtained a 'Schwinger proper-time' form of the calculation such as that used in ref. [58].)

As with the open string case we can adapt the cycles rules to include other particles. For vectors and scalars in the loop, we can find rules by using compactification arguments again $[82,65]$. The result for a vector is that we must apply the open string scalar rules to one component and the open string vector rules to the other. For a scalar we simply apply open string scalar rules to both components.

For the fermions, we must look to superstrings for our motivation. The Dirac ( $\left.\operatorname{spin}-\frac{1}{2}\right)$ fermion has the rules one, would expect after examining the vector and scalar cases: we apply open string scalar rules to one component and open string fermion rules to the other. For a Rarita-Schwinger ( $\operatorname{spin}-\frac{3}{2}$ ) fermion we might expect to apply open string fermion and open string vector rules to each component respectively. This is almost right - in fact, like the graviton, this includes slightly more than the particle required. In this case we will have one spin- $\frac{3}{2}$ and one spin $-\frac{1}{2}$ particle. So the Rarita-Schwinger fermion will be found by subtracting the Dirac result from this. 
Given these adjustments, we can now construct a full set of rules for graviton scattering amplitudes which, as in the QCD case, can be used without knowledge of their string theory source.

\subsection{Gravity Rules}

As with the QCD rules, our starting point for an amplitude is to draw all the relevant diagrams - that is'all those constructed from $\phi^{3}$ vertices. (Excluding tadpoles and diagrams with the loop isolated on an external leg.) The difference here is that we must include diagrams with all orderings of the external labels. The internal lines are labelled according to the pinch rules - at a three point vertex the internal leg is given the label of the most clockwise of the two outer legs. The contribution from each labelled $n$-point $\phi^{3}$-like diagram with $n_{\ell}$ legs attached to the loop is

$$
\begin{array}{r}
\mathcal{D}_{S}=i \frac{(-\kappa)^{n}}{(4 \pi)^{2-\epsilon}} \int_{0}^{1} d x_{i_{n_{\ell}-1}} \int_{0}^{x_{i_{n_{\ell}-1}}} d x_{i_{n_{\ell}-2}} \cdots \int_{0}^{x_{i_{2}}} d x_{i_{1}} \int_{0}^{\infty} d T T^{n_{\ell}-3+\epsilon} \\
\quad \times \exp \left(-T \sum_{l<m}^{n_{\ell}} P_{i_{l}} \cdot P_{i_{m}} x_{i_{m} i_{l}}\left(1-x_{i_{m} i_{\ell}}\right)\right) K_{\text {red }}\left(x_{i_{1}}, \ldots, x_{i_{n_{\ell}}}, T\right)
\end{array}
$$

(where $n_{l}$ is the number of legs attached to the loop). In order to evaluate $K_{\text {red }}$, we start with the graviton kinematic expression

$$
\begin{aligned}
\mathcal{K}=\int & \prod_{i=1}^{n} d x_{i} d \bar{x}_{i} \prod_{i<j}^{n} \exp \left[k_{i} \cdot k_{j} G_{B}^{i j}\right] \exp \left[\left(k_{i} \cdot \epsilon_{j}-k_{j} \cdot \epsilon_{i}\right) \dot{G}_{B}^{i j}-\epsilon_{i} \cdot \epsilon_{j} \ddot{G}_{B}^{i j}\right] \\
& \times\left.\exp \left[\left(k_{i} \cdot \bar{\epsilon}_{j}-k_{j} \cdot \bar{\epsilon}_{i}\right) \dot{\bar{G}}_{B}^{i j}-\bar{\epsilon}_{i} \cdot \bar{\epsilon}_{j} \ddot{\bar{G}}_{B}^{i j}\right] \exp \left[-\left(\epsilon_{i} \cdot \bar{\epsilon}_{j}+\epsilon_{j} \cdot \bar{\epsilon}_{i}\right) H_{B}^{i j}\right]\right|_{\text {multi-linear }}
\end{aligned}
$$

We have factorised the graviton polarisation tensor as described earlier; we will recover it at the end by taking $\epsilon_{i}^{\mu} \bar{\epsilon}_{i}^{\nu} \rightarrow \epsilon_{i}^{\mu \nu} . G_{B}$ 's can be regarded as nothing more than 'Feynman 'parameter functions'. $\dot{G}_{B}$ and $\ddot{G}_{B}$ are derivatives of these with respect to left-moving variables (e.g., $\dot{G}_{B}^{12}=\frac{1}{2} \frac{\partial G_{B}^{12}}{\partial x_{1}}$ ) and $\dot{\bar{G}}_{B}$ and $\ddot{G}_{B}$ are derivatives with respect to right-movers (e.g., $\dot{\bar{G}}_{B}^{12}=\frac{1}{2} \frac{\partial G_{B}^{12}}{\partial \bar{x}_{1}}$ ). The term $H_{B}^{i j}$ is the derivative of the Green function with respect 
to one left-moving and one right-moving variable. The functions $G_{B}^{i j}, \ddot{G}_{B}^{i j}$ and $H_{B}^{i j}$ are symmetric in the $i$ and $j$ indices while $\dot{G}_{B}$ is antisymmetric.

We can begin by using spinor-helicity simplifications where possible (appendix III). This entails choosing a particular helicity configuration and relevant reference momenta. Repeating the calculation for all helicity configurations will give us the complete amplitude. Though it is not a necessary part of the rules, the spinor-helicity method will, with a good choice of referencè momenta, remove a number of terms in (5.15) and cut down the work in evaluating the expression.

The first step in the rules is to remove all of the $\ddot{\bar{G}}_{B}^{i j}$ and $\ddot{G}_{B}^{i j}$ by integrating the kinematic expression by parts with respect to the variables $x_{i}$ and $\bar{x}_{i}$ "where necessary. While carrying out this process we must be careful to take into account the cross-terms where a left-mover derivative hits right-mover terms, and vice versa. This can be done by using the results

$$
\begin{array}{cc}
\frac{\partial}{\partial \bar{x}_{k}} \dot{G}_{B}^{i j}=\delta_{k i} H_{B}^{i j}-\delta_{k j} H_{B}^{i j} & \frac{\partial}{\partial x_{k}} \dot{\bar{G}}_{B}^{i j}=\delta_{k i} H_{B}^{i j}-\delta_{k j} H_{B}^{i j} \\
\frac{\partial}{\partial \bar{x}_{k}} \ddot{G}_{B}^{i j}=0 & \frac{\partial}{\partial x_{k}} \ddot{\bar{G}}_{B}^{i j}=0
\end{array}
$$

For example, if the expression

$$
\int \prod_{i=1}^{4} d x_{i} d \bar{x}_{i} \prod_{i<j}^{4} \exp \left[k_{i} \cdot k_{j} G_{B}^{i j}\right] \dot{G}_{B}^{34} \ddot{G}_{B}^{12} \dot{\bar{G}}_{B}^{13}\left(\dot{\bar{G}}_{B}^{34}\right)^{2}
$$

is integrated by parts with respect to $x_{1}$, the result is

$$
\begin{aligned}
\int \prod_{i=1}^{4} d x_{i} d \bar{x}_{i} \prod_{i<j}^{4} \exp \left[k_{i} \cdot k_{j} G_{B}^{i j}\right] \dot{G}_{B}^{34} \dot{G}_{B}^{12}\left(\dot{\bar{G}}_{B}^{34}\right)^{2} \\
\times\left(\left(k_{1} \cdot k_{2} \dot{G}_{B}^{12}+k_{1} \cdot k_{3} \dot{G}_{B}^{13}+k_{1} \cdot k_{4} \dot{G}_{B}^{14}\right) \dot{\bar{G}}_{B}^{13}+H_{B}^{13}\right)
\end{aligned}
$$

(Repeated integrations by parts may be needed to remove the $\ddot{G}_{B}$ and $\ddot{\bar{G}}_{B}$ 's completely.) Once all $\ddot{G}_{B}$ and $\ddot{\bar{G}}_{B}$ 's have been dealt with we can drop the integral and exponential terms, leaving us with a function consisting of $\dot{G}_{B}$ and $\dot{\bar{G}}_{B}$ 's. Using this function, we can 
go on to find an expression for $K_{\text {red }}$ for each diagram in turn. We begin with the pinch contribution: For every tree attached to the loop we make the substitution in fig.5.A. As in the QCD case, we begin with the outermost tree vertices and work inwards applying the rule where appropriate.

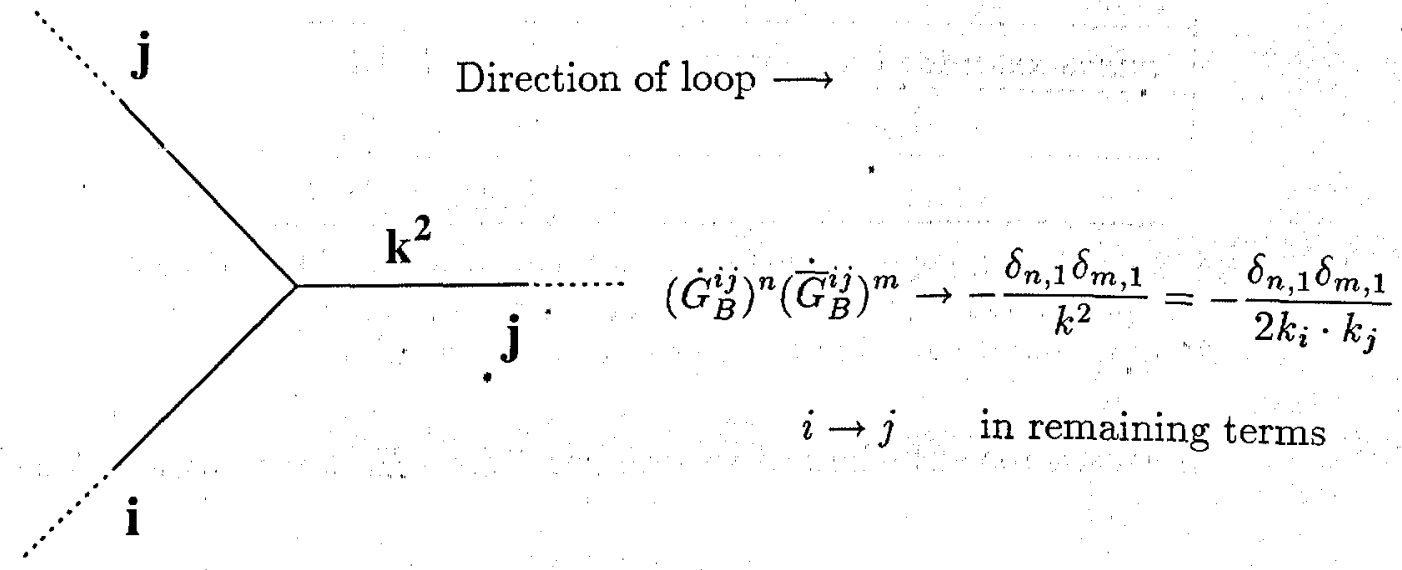

Figure 5.A: The Tree Rules. Note that leg i * appears before leg $\mathrm{j}$ in the (clockwise) cyclic ordering.

Once the tree rules have been carried out for a diagram, we apply the loop rules. The first step is to replace all occurrences of $H_{B}$ with

$$
H_{B}^{i j} \longrightarrow \frac{1}{2 T}
$$

We then turn to the remaining derivatives of $G_{B}$. From earlier discussions, we know that the rules for $\dot{G}_{B}$ and $\dot{\bar{G}}_{B}$ replacements will depend on the particles which are circulating in the loop. As suggested in the derivation, our building blocks are the open string loop rules. We can obtain the full spectrum of particles $($ spin $\leq 2)$ 'in the loop' by applying combinations of the QCD rules to the left and right components of the kinematic function.

In table 5.1, we give the combinations required for the different particles. By $[X, Y]$ we mean that rules $X$ should be applied to the $\dot{G}_{B}$ 's and rules $Y$ should be applied to the $\dot{G}_{B}$ 's. $S, F$ and $V$ represent the scalar, fermion and vector loop rules respectively. (The vector rules we require here are those for a single vector state, equs. (4.46) and (4.47).) 


\begin{tabular}{|c|c|}
\hline Substitution & Particle Content \\
\hline $2[S, S]$ & complex scalar \\
\hline$-2[S, F]$ & Weyl Fermion \\
\hline $2[S, V]$ & Vector \\
\hline$-4[V, F]$ & gravitino and Weyl Fermion \\
\hline $4[V, V]$ & graviton and complex scalar \\
\hline $4[V, V]-2[S, S]$ & graviton \\
\hline$-4[V, F]+2[S, F]$ & gravitino \\
\hline
\end{tabular}

Table 5.1: Applying the substitution rules on the left corresponds to having the particle content on the right circulating in the loop. $[x, y]$ denotes applying substitution rules $x$ and $y$ to barred and unbarred terms, respectively.

The scalar contribution, $S$, simply requires us to make the substitution

$$
\begin{aligned}
& \dot{G}_{B}^{i j} \longrightarrow \frac{1}{2}\left(-\operatorname{sign}\left(x_{i j}\right)+2 x_{i j}\right) \\
& \dot{\bar{G}}_{B}^{i j} \longrightarrow \frac{1}{2}\left(-\operatorname{sign}\left(x_{i j}\right)+2 x_{i j}\right)
\end{aligned} .
$$

We know that $F$ and $V$ each produce two types of contribution; scalar, $S$, and cycle. A cycle contribution is found whenever we have a 'cycle' of $\dot{G}_{B}$ or $\dot{G}_{B}$ 's:

$$
\dot{G}_{B}^{i_{1} i_{2}} \dot{G}_{B}^{i_{2} i_{3}} \ldots \dot{G}_{B}^{i_{n-1} i_{n}} \dot{G}_{B}^{i_{n} i_{1}} \text { or } \dot{\bar{G}}_{B}^{i_{1} i_{2}} \dot{\bar{G}}_{B}^{i_{2} i_{3}} \ldots \dot{\bar{G}}_{B}^{i_{n-1} i_{n}} \dot{\bar{G}}_{B}^{i_{n} i_{1}}
$$

Let us write the cycle contributions as $C_{V}$ and $C_{F}$. So,

$$
\begin{aligned}
& F=S+C_{F} \\
& V=S+C_{V}
\end{aligned}
$$

For $C_{V}$, the substitution rules are

$$
\begin{gathered}
\dot{G}_{B}^{i_{1} i_{2}} \dot{G}_{B}^{i_{2} i_{1}} \rightarrow 1 \\
\dot{G}_{B}^{i_{1} i_{2}} \dot{G}_{B}^{i_{2} i_{3}} \ldots \dot{G}_{B}^{i_{m-1} i_{m}} \dot{G}_{B}^{i_{m} i_{1}} \rightarrow 1 / 2 \quad(m>2)
\end{gathered}
$$

where all the cycles must follow the ordering of the legs and only one cycle at a time may contribute to any term. Once these substitutions have been made all remaining $\dot{G}_{B}$ 's should be replaced as in equ. (5.20). 
For $C_{F}$ the following substitution is made

$$
\dot{G}_{B}^{i_{1} i_{2}} \dot{G}_{B}^{i_{2} i_{3}} \ldots \dot{G}_{B}^{i_{m-1} i_{m}} \dot{G}_{B}^{i_{m} i_{1}} \rightarrow-(-1 / 2)^{m} \prod_{k=1}^{m} \operatorname{sign}\left(x_{i_{k} i_{k+1}}\right)
$$

In contrast to the $V$ rules, all cycles contribute in the $F$ case regardless of ordering. Also, all combinations of one or more cycles from each term contribute. Again, once these substitutions have been made all remaining $\dot{G}_{B}$ 's should be replaced as in (5.20).

Once we have carried out the pinch and loop rules for the diagram and particle content we are considering, we obtain an expression for $K_{\text {red. }}$. We then insert this into (5.14) and carry out the integrations. In contrast to the QCD case, we have an extra $T$ integration which must be evaluated. If we do this first we will find a similar denominator to the QCD case, possibly with a differing exponent. (After carrying out the $T$ integration, a substitution $x_{i}=\sum_{j=1}^{i} a_{j}$ will show that again we have recovered a Feynman parameter integration. $T$ is, in some senses, the Schwinger proper time.) Once we have carried out all integrations, we can sum the results from all diagrams to obtain the full amplitude.

One reason for expressing the loop rules in the form (5.22) is that it allows us to make explicit the simplifications in a SUSY decomposition (appendix V). This involves calculating amplitudes for specific SUSY multiplets rather than for individual particles. The individual contributions can then be reconstructed from the SUSY results. In this way, we can exploit SUSY simplifications during the calculational process; they are seen as cancellations between common contributions in the multiplets.

\begin{tabular}{|c|c|c|c|c|c|}
\hline$N$ & scalars & spin- $1 / 2$ & spin- 1 & spin-3/2 & spin-2 \\
\hline$N=0$ & 1 & & & & \\
\hline$N=1$ & 1 & 1 & & & \\
\hline$N=4$ & 3 & 4 & 1 & & \\
\hline$N=6$ & 10 & 15 & 6 & 1 & \\
\hline$N=8$ & 35 & 56 & 28 & 8 & 1 \\
\hline
\end{tabular}

Table 5.2: Particle content of the supersymmetric multiplets we consider. Scalars are complex, and the fermions are Weyl. 
Here, we chose to calculate amplitudes for the $N=0,1,4,6,8$ multiplets centred around the scalar (ie, those in table 5.2). The cancellations for these multiplets leave a simple contribution to be calculated and a reduced degree of Feynman parameter polynomial (see table '5.3, and note that in the combination $C_{V}-4 C_{F}$ the 2- and 3-cycle contributions cancel). Clearly, this will be a particular efficient way to carry out the one loop amplitude calculations.

\begin{tabular}{|c|c|c|}
\hline$N$ & Contribution & Degree \\
\hline$N=0$ & $2[S, S]$ & $2 n$ \\
\hline$N=1$ & $2\left[C_{F}, S\right]$ & $2 n-2$ \\
\hline$N=4$ & $2\left[C_{V}-4 C_{F}, S\right]$ & $2 n-4$ \\
\hline$N=6$ & $-4\left[C_{V}-4 C_{F}, C_{F}\right]$ & $2 n-6$ \\
\hline$N=8$ & $4\left[C_{V}-4 C_{F}, C_{V}-4 C_{F}\right]$ & $2 n-8$ \\
\hline
\end{tabular}

Table 5.3: The String rules appropriate for the multiplet are given and the degree of the Feynman parameter polynomial for an $n$-point loop integral.

Using the string rules in this way gives a further simplification compared to Feynman techniques.

\subsection{Sample Calculation ${ }^{\dagger}$}

Let us look now at an example of a typical calculation using this technique. We will choose the four-point amplitude with helicity configuration $A^{1-\mathrm{loop}}\left(1-2^{+} 3^{+} 4^{+}\right)$; the gravity equivalent to the $\mathrm{QCD}$ example in the previous chapter.

We begin by identifying the relevant diagrams; these are depicted fig.5.B. These are essentially the same as those in the QCD example, but recall that we must now include all orderings of the external legs.

We go on to look at the use of the spinor-helicity technique. The most suitable choice of helicity reference momenta for this configuration is $\left(k_{4}, k_{1}, k_{1}, k_{1}\right)$. This allows us to

† This was originally calculated in ref. [31] 


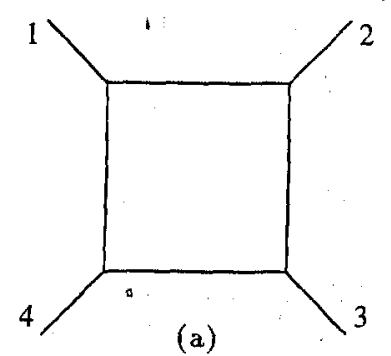

(a)
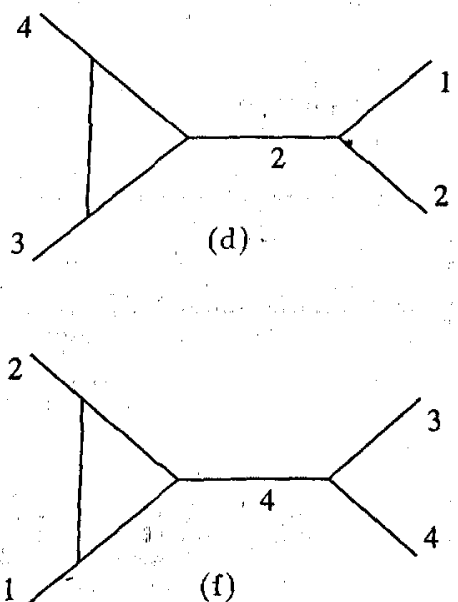

(I)

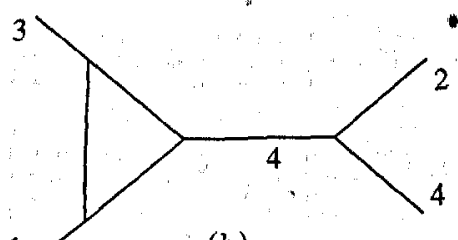

(h)

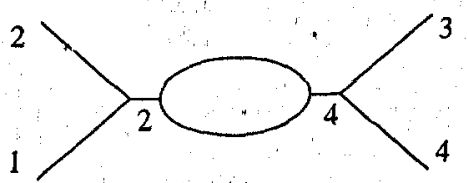

(i)
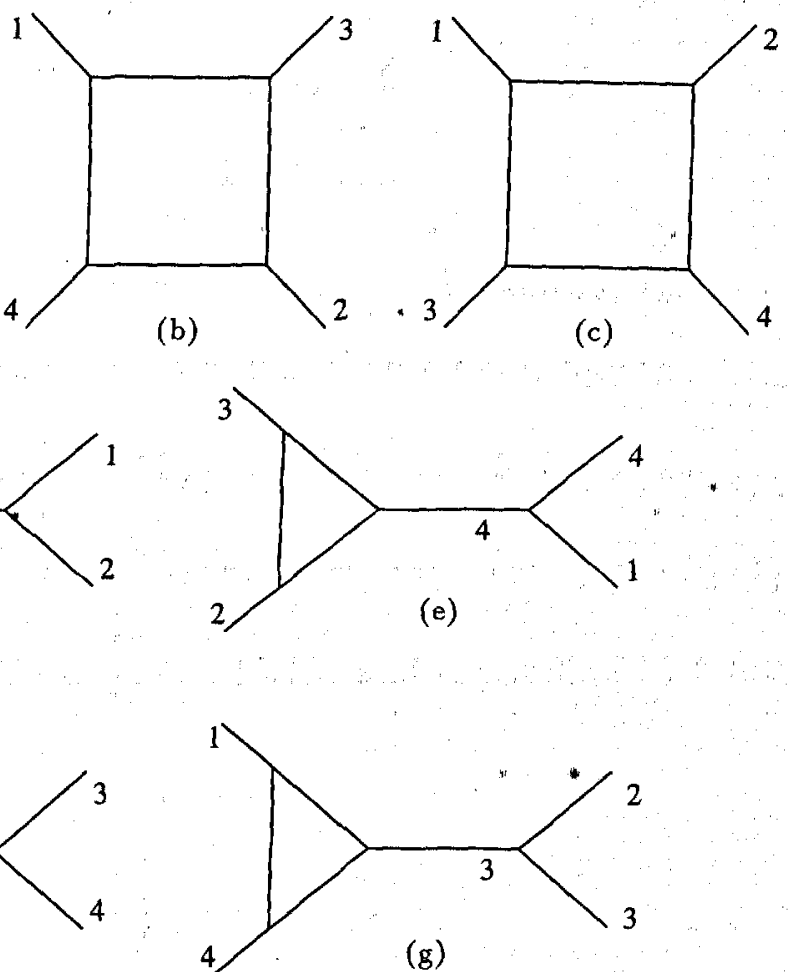

(g)
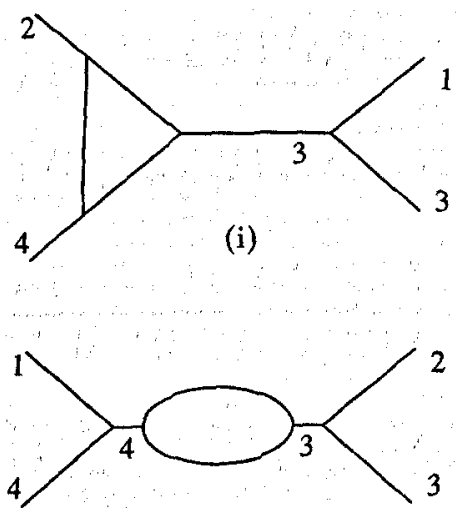

(k)

Figure 5.B: Relevant diagrams for the $A^{1-\operatorname{loop}}\left(1^{-} 2^{+} 3^{+} 4^{+}\right)$ graviton calculation. 
deduce the formulae:

$$
\begin{array}{ccc}
\epsilon_{i} \cdot \epsilon_{j}=0 & \bar{\epsilon}_{i} \cdot \bar{\epsilon}_{j}=0 & \forall i, j \\
\epsilon_{i} \cdot \bar{\epsilon}_{j}=0 & \forall i, j \\
k_{1} \cdot \epsilon_{j}=0 & k_{1} \cdot \bar{\epsilon}_{j}=0 & \forall j \\
k_{4} \cdot \epsilon_{1}=0 & k_{4} \cdot \bar{\epsilon}_{1}=0 . &
\end{array}
$$

(Recall that we can factorise the polarisation tensor as two vectors, $\epsilon_{\mu \nu}=\epsilon_{\mu} \bar{\epsilon}_{\nu}$.) If we combine these results with momentum conservation, then we can equate

$$
\begin{array}{llll}
k_{2} \cdot \epsilon_{1}=-k_{3} \cdot \epsilon_{1} & k_{3} \cdot \epsilon_{2}=-k_{4} \cdot \epsilon_{2} \quad & k_{2} \cdot \bar{\epsilon}_{1}=-k_{3} \cdot \bar{\epsilon}_{1} & k_{3} \cdot \bar{\epsilon}_{2}=-k_{4} \cdot \bar{\epsilon}_{2} \\
k_{2} \cdot \epsilon_{3}=-k_{4} \cdot \epsilon_{3} & k_{2} \cdot \epsilon_{4}=-k_{3} \cdot \epsilon_{4} & k_{2} \cdot \bar{\epsilon}_{3}=-k_{4} \cdot \bar{\epsilon}_{3} & k_{2} \cdot \bar{\epsilon}_{4}=-k_{3} \cdot \bar{\epsilon}_{4} .
\end{array}
$$

Using these results in the kinematic expression (5.15) and expanding to fulfil the multilinear condition gives us

$$
\begin{aligned}
\mathcal{K}=\int & \prod_{i=1}^{4} d x_{i} d \bar{x}_{i} \prod_{i<j}^{4} \exp \left[k_{i} \cdot k_{j} G_{B}^{i j}\right] \\
& \times k_{2} \cdot \epsilon_{1} k_{3} \cdot \epsilon_{2} k_{2} \cdot \epsilon_{3} k_{2} \cdot \epsilon_{4} k_{2} \cdot \bar{\epsilon}_{1} k_{3} \cdot \bar{\epsilon}_{2} k_{2} \cdot \bar{\epsilon}_{3} k_{2} \cdot \bar{\epsilon}_{4} \\
\times & \left.\times\left(-\dot{G}_{B}^{13}+\dot{G}_{B}^{12}\right)\left(-\dot{G}_{B}^{24}+\dot{G}_{B}^{23}\right)\left(\dot{G}_{B}^{23}+\dot{G}_{B}^{34}\right)\left(\dot{G}_{B}^{24}-\dot{G}_{B}^{34}\right)\right] \\
\times & {\left[\left(-\dot{\bar{G}}_{B}^{13}+\dot{\bar{G}}_{B}^{12}\right)\left(-\dot{\bar{G}}_{B}^{24}+\dot{\bar{G}}_{B}^{23}\right)\left(\dot{\bar{G}}_{B}^{23}+\dot{\bar{G}}_{B}^{34}\right)\left(\dot{\bar{G}}_{B}^{24}-\dot{\bar{G}}_{B}^{34}\right)\right] } \\
=\int & \prod_{i=1}^{4} d x_{i} d \bar{x}_{i} \prod_{i<j}^{4} \exp \left[k_{i} \cdot k_{j} G_{B}^{i j}\right]\left(\frac{s^{2} t}{4} \frac{[24]^{2} t s^{2}}{[12]\langle 23\rangle\langle 34\rangle[41]}\right)^{2} \\
\times & {\left[\left(-\dot{G}_{B}^{13}+\dot{G}_{B}^{12}\right)\left(-\dot{G}_{B}^{24}+\dot{G}_{B}^{23}\right)\left(\dot{G}_{B}^{23}+\dot{G}_{B}^{34}\right)\left(\dot{G}_{B}^{24}-\dot{G}_{B}^{34}\right)\right] } \\
\times & {\left[\left(-\dot{\bar{G}}_{B}^{13}+\dot{\bar{G}}_{B}^{12}\right)\left(-\dot{\bar{G}}_{B}^{24}+\dot{\bar{G}}_{B}^{23}\right)\left(\dot{\bar{G}}_{B}^{23}+\dot{\bar{G}}_{B}^{34}\right)\left(\dot{\bar{G}}_{B}^{24}-\dot{\bar{G}}_{B}^{34}\right)\right] }
\end{aligned}
$$

Note that this is simply a double copy of the kinematic expression in the QCD example, so by comparison with that case we know immediately that there is no contribution from pinches (e), (f), (g), (h), (j) and (k). In this case, however, we are left with five other diagrams which we must consider. We have more here because we are considering all 
orderings of the external legs, so there are three boxes to be considered rather than the one in the QCD case, plus an extra pinch diagram.

Let us look first at the scalar contributions from the contributing diagrams. The boxes have no pinches, so we can look at the loop rules straight away. We begin with (a). The scalar loop contributions depend on the ordering of the external legs through the $\operatorname{sign}\left(x_{i j}\right)$ in (5.20). (a) has the ordering $0<x_{1}<x_{2}<x_{3}<x_{4}=1$, which is the same as the QCD box in the previous example. Hence, the reduced kinematic factor which we obtain is precisely the square of (4.54):

$$
K_{\text {red }}^{(a)}=\frac{\kappa^{4}}{16}\left(\frac{[24]^{2} t s^{2}}{[12]\langle 23\rangle\langle 34\rangle[41]}\right)^{2}\left(\left(x_{2}-x_{3}\right)\left(x_{3}-1\right) x_{2}\left(x_{2}-x_{3}\right)\right)^{2}
$$

Substituting this into (5.15), and carrying out the $\mathrm{T}$ integration, gives us

$$
\begin{aligned}
\mathcal{D}_{s}^{(a)}= & \frac{\Gamma\left(n_{l}-2+\frac{1}{2} \epsilon\right)}{(4 \pi)^{2-\epsilon / 2}} \frac{1}{16}\left(\frac{[24]^{2} t^{2} s}{[12]\langle 23\rangle\langle 34\rangle[41]}\right)^{2} \\
& \times \int_{0}^{1} d x_{3} \int_{0}^{x_{3}} d x_{2} \int_{0}^{x_{2}} d x_{1} \frac{\left(x_{2}-x_{3}\right)^{4}\left(x_{3}-1\right)^{2} x_{2}^{2}}{\left(-s x_{1}\left(x_{3}-x_{2}\right)-t\left(x_{2}-x_{1}\right)\left(1-x_{3}\right)\right)^{2+\epsilon / 2}}
\end{aligned}
$$

Which, use the Feynman parameter form is

$$
\begin{array}{r}
\mathcal{D}_{s}^{(a)}=\frac{\Gamma\left(2+\frac{1}{2} \epsilon\right)}{(4 \pi)^{2-\epsilon / 2}} \frac{\kappa^{4}}{16}\left(\frac{[24]^{2} t s^{2}}{[12]\langle 23\rangle\langle 34\rangle[41]}\right)^{2} \\
\times \int_{0}^{1} \prod_{i=1}^{4} d a_{i} \delta\left(1-\sum_{i=1}^{4} a_{i}\right) \frac{\left(a_{3}^{2} a_{4}\left(a_{1}+a_{2}\right)\right)^{2}}{\left(-s a_{1} a_{3}-t a_{2} a_{4}\right)^{2+\epsilon / 2}}
\end{array}
$$

We obtain from this

$$
\mathcal{D}_{s}^{(a)}=r_{\Gamma} \frac{i \kappa^{4}}{(4 \pi)^{2}} \frac{1}{840 s t}\left(\frac{[24]^{2} t s^{2}}{[12]\langle 23\rangle\langle 34\rangle[41]}\right)^{2}
$$

where $r_{\Gamma}$ is given by

$$
r_{\Gamma}=\frac{\Gamma^{2}(1-\epsilon) \Gamma(1+\epsilon)}{\Gamma(1-2 \epsilon)}
$$

For (b); the ordering is $0<x_{1}<x_{3}<x_{2}<x_{4}=1$ which leads to a reduced kinematic expression (after loop substitutions) of

$$
K_{\text {red }}^{(b)}=\frac{1}{16}\left(\frac{[24]^{2} t s^{2}}{[12]\langle 23\rangle\langle 34\rangle[41]}\right)^{2}\left(\left(x_{3}-x_{2}\right)\left(2-x_{3}\right)\left(-1+x_{2}\right)\left(x_{2}-x_{3}\right)\right)^{2}
$$


The relevant denominator in the integral is now $\left(-u x_{1}\left(x_{3}-x_{2}\right)-t\left(x_{2}-x_{1}\right)\left(1-x_{3}\right)\right)^{2+\epsilon / 2}$. So we obtain the integral (in Feynman parameter form)

$$
\begin{aligned}
\mathcal{D}_{s}^{(b)}=\frac{\Gamma\left(2+\frac{1}{2} \epsilon\right)}{(4 \pi)^{2-\epsilon / 2}} \frac{\kappa^{4}}{16}\left(\frac{[24]^{2} t s^{2}}{[12]\langle 23\rangle\langle 34\rangle[41]}\right)^{2} & \\
& \times \int_{0}^{1} \prod_{i=1}^{4} d a_{i} \delta\left(1-\sum_{i=1}^{4} a_{i}\right) \frac{\left(a_{3}^{2} a_{4}\left(a_{1}+a_{2}-2\right)\right)^{2}}{\left(-u a_{1} a_{3}-t a_{2} a_{4}\right)^{2+\epsilon / 2}}
\end{aligned}
$$

Which gives

$$
\mathcal{D}_{s}^{(b)}=r_{\mathrm{\Gamma}} \frac{i \kappa^{4}}{(4 \pi)^{2}} \frac{1}{840 u t}\left(\frac{[24]^{2} t s^{2}}{[12]\langle 23\rangle\langle 34\rangle[41]}\right)^{2}
$$

The final box is (c), with ordering $0<x_{1}<x_{2}<x_{4}<x_{3}=1$. The reduced kinematic expression for this is

$$
K_{\text {red }}^{(c)}=\frac{1}{16}\left(\frac{[24]^{2} t s^{2}}{[12]\langle 23\rangle\langle 34\rangle[41]}\right)^{2}\left(\left(1-x_{2}\right)\left(x_{4}-1\right)\left(x_{2}-\dot{x}_{4}\right) x_{2}\right)^{2}
$$

and the denominator required is $\left(-s x_{1}\left(x_{4}-x_{2}\right)-u\left(x_{2}-x_{1}\right)\left(1-x_{4}\right)\right)^{2+\epsilon / 2}$. From these we obtain the Feynman parameter integral

$$
\begin{array}{r}
\mathcal{D}_{s}^{(c)}=\frac{\Gamma\left(2+\frac{1}{2} \epsilon\right)}{(4 \pi)^{2-\epsilon / 2}} \frac{\kappa^{4}}{16}\left(\frac{[24]^{2} t s^{2}}{[12]\langle 23\rangle\langle 34\rangle[41]}\right)^{2} \\
\times \int_{0}^{1} \prod_{i=1}^{4} d a_{i} \delta\left(1-\sum_{i=1}^{4} a_{i}\right) \frac{\left(a_{3}^{2} a_{4}\left(a_{1}+a_{2}-2\right)\right)^{2}}{\left(-s a_{1} a_{3}-u a_{2} a_{4}\right)^{2+\epsilon / 2}}
\end{array}
$$

Giving the result

$$
\mathcal{D}_{s}^{(c)}=r_{\Gamma} \frac{i \kappa^{4}}{(4 \pi)^{2}} \frac{1}{252 s u}\left(\frac{[24]^{2} t s^{2}}{[12]\langle 23\rangle\langle 34\rangle[41]}\right)^{2}
$$

Now we must look at the pinch diagrams. We begin with (d), the 1-2 pinch. Recall that we must now look for terms containing exactly one $\dot{G}_{B}^{12}$ and one $\dot{\bar{G}}_{B}^{12}$; the substitution rule in this case is

$$
\left(\dot{G}_{B}^{12}\right)^{n}\left(\dot{G}_{B}^{12}\right)^{m}=\frac{\delta_{m, 1} \delta_{n, 1}}{2 k_{1} \cdot k_{2}}=\frac{\delta_{m, 1} \delta_{n, 1}}{s}
$$


and change the indices $1 \rightarrow 2$ in remaining terms. This gives

$$
\begin{array}{r}
\frac{1}{16}\left(\frac{[24]^{2} t s^{2}}{[12]\langle 23\rangle\langle 34\rangle[41]}\right)^{2} \frac{1}{s}\left(\dot{G}_{B}^{23}-\dot{G}_{B}^{24}\right)\left(\dot{G}_{B}^{23}+\dot{G}_{B}^{34}\right)\left(\dot{G}_{B}^{24}-\dot{G}_{B}^{34}\right) \\
\times\left(\dot{\bar{G}}_{B}^{23}-\dot{\bar{G}}_{B}^{24}\right)\left(\dot{\bar{G}}_{B}^{23}+\dot{\bar{G}}_{B}^{34}\right)\left(\dot{\bar{G}}_{B}^{24}-\dot{\bar{G}}_{B}^{34}\right) \\
=\frac{1}{16 s}\left(\frac{[24]^{2} t s^{2}}{[12]\langle 23\rangle\langle 34\rangle[41]}\right)^{2}\left(\left(x_{3}-1\right) x_{2}\left(x_{2}-x_{3}\right)\right)^{2}
\end{array}
$$

With the relevant denominator, this becomes

$$
\begin{aligned}
& \mathcal{D}_{s}^{(d)}=\frac{\Gamma\left(1+\frac{1}{2} \epsilon\right)}{(4 \pi)^{2-\epsilon / 2}} \frac{\kappa^{4}}{16 s} \frac{[24]^{2} t s^{2}}{[12]\langle 23\rangle\langle 34\rangle[41]} \\
& \dot{x} \int_{0}^{1} \prod_{i=2}^{4} d a_{i} \delta\left(1-\sum_{i=2}^{4} a_{i}\right) \frac{\left(a_{3} a_{4}\left(a_{2}-2\right)\right)^{2}}{\left(-s a_{2} a_{3}\right)^{1+\epsilon / 2}}
\end{aligned}
$$

where, as usual, we have converted to Feynman parameters. This evaluates to

$$
\mathcal{D}_{s}^{(d)}=\frac{i \kappa^{4}}{(4 \pi)^{2}} \frac{1}{360} \frac{1}{s^{2}}\left(\frac{[24]^{2} t s^{2}}{[12]\langle 23\rangle\langle 34\rangle[41]}\right)^{2}
$$

Finally, we have the 1-3 pinch diagram (i). After applying the pinch rules we obtain the expression

$$
\begin{aligned}
\frac{1}{16}\left(\frac{[24]^{2} t s^{2}}{[12]\langle 23\rangle\langle 34\rangle[41]}\right)^{2} \frac{1}{u}\left(\dot{G}_{B}^{23}-\dot{G}_{B}^{24}\right)\left(\dot{G}_{B}^{23}+\dot{G}_{B}^{34}\right)\left(\dot{G}_{B}^{24}-\dot{G}_{B}^{34}\right) & \times\left(\dot{\bar{G}}_{B}^{23}-\dot{\bar{G}}_{B}^{24}\right)\left(\dot{\bar{G}}_{B}^{23}+\dot{\bar{G}}_{B}^{34}\right)\left(\dot{\bar{G}}_{B}^{24}-\dot{\bar{G}}_{B}^{34}\right) \\
= & \frac{1}{16 u}\left(\frac{[24]^{2} t s^{2}}{[12]\langle 23\rangle\langle 34\rangle[41]}\right)^{2}\left(\left(x_{3}-1\right) x_{2}\left(x_{2}-x_{3}\right)\right)^{2}
\end{aligned}
$$

The denominator which require in this case is $\left(-u a_{2} a_{3}\right)^{1+\epsilon / 2}$. Clearly we have the same integral as in (d) up to a factor of $(s / u)^{2}$. Hence we find

$$
\mathcal{D}_{s}^{(i)}=\frac{i \kappa^{\dot{4}}}{(4 \pi)^{2}} \frac{1}{360} \frac{1}{u^{2}}\left(\frac{[24]^{2} t s^{2}}{[12]\langle 23\rangle\langle 34\rangle[41]}\right)^{2}
$$

Summing the various contributions gives us the final result of

$$
A\left(1^{-}, 2^{+}, 3^{+}, 4^{+}\right)=N_{s} \frac{i \kappa^{4}}{(4 \pi)^{2}}\left(\frac{s t}{u}\right)^{2}\left(\frac{[24]^{2}}{[12]\langle 23\rangle\langle 34\rangle[41]}\right)^{2} \frac{\left(s^{2}+s t+t^{2}\right)}{5760}
$$




\subsection{Results}

Here we give a complete set of results for 4-graviton amplitudes. We express them using the spinor-helicity terminology, and making use of the SUSY decomposition described in appendix IV.

For the $(-,+,+,+)$ and $(+,+,+,+)$ one-loop amplitudes all the supersymmetric components in the decomposition vanish due to supersymmetric Ward identities. This is related to the fact that all cycles vanish, so that the contribution for every particle is just a multiple of the scalar one. The results are

$$
A\left(1^{-}, 2^{+}, 3^{+}, 4^{+}\right)=N_{s} \frac{i \kappa^{4}}{(4 \pi)^{2}}\left(\frac{s t}{u}\right)^{2}\left(\frac{[24]^{2}}{[12]\langle 23\rangle\langle 34\rangle[41]}\right)^{2} \frac{\left(s^{2}+s t+t^{2}\right)}{5760}
$$

and

$$
A\left(1^{+}, 2^{+}, 3^{+}, 4^{+}\right)=-N_{s} \frac{i \kappa^{4}}{(4 \pi)^{2}}\left(\frac{s t}{\langle 12\rangle\langle 23\rangle\langle 34\rangle\langle 41\rangle}\right)^{2} \frac{\left(s^{2}+. s t+t^{2}\right)}{1920}
$$

where

$$
\therefore N_{s}=N_{b}-N_{f}
$$

the number of bosonic states in the loop minus the number of fermionic states, $s=\left(k_{1}+\right.$ $\left.k_{2}\right)^{2}, t=\left(k_{1}+k_{4}\right)^{2}$ and $u=\left(k_{1}+k_{3}\right)^{2}$. So, for instance, since a graviton is made up of two helicity states the amplitudes for pure gravity are found by putting $N_{s}=2$ in the above expressions.

For the $A\left(1^{-}, 2^{-}, 3^{+}, 4^{+}\right)$amplitude, none of the cycle terms vanish. We express their contributions using the supersymmetric decomposition given in the previous section. The (complex) scalar amplitude is

$$
\begin{array}{r}
A^{[0]}\left(1^{-}, 2^{-}, 3^{+}, 4^{+}\right)=-\frac{F u^{3} t^{3}\left(\ln (-t /-u)^{2}+\pi^{2}\right)}{s^{8}} \\
+\frac{F(t-u)\left(t^{4}+9 u t^{3}+46 u^{2} t^{2}+9 u^{3} t+u^{4}\right) \ln (-t /-u)}{30 s^{7}} \\
+\frac{F\left(2 t^{4}+23 u t^{3}+222 u^{2} t^{2}+23 u^{3} t+2 u^{4}\right)}{180 s^{6}}
\end{array}
$$


where $F$ is

$$
\frac{i \kappa^{4}(4 \pi)^{\epsilon} r_{\Gamma}}{16(4 \pi)^{2}}\left(\frac{s t\langle 12\rangle^{4}}{\langle 12\rangle\langle 23\rangle\langle 34\rangle\langle 41\rangle}\right)^{2}=\frac{s t u \kappa^{2}(4 \pi)^{\epsilon} r_{\Gamma}}{4(4 \pi)^{2}} A^{\text {tree }}\left(1^{-}, 2^{-}, 3^{+}, 4^{+}\right)
$$

and $A^{\text {tree }}\left(1^{-}, 2^{-}, 3^{+}, 4^{+}\right)$is

$$
A^{\text {tree }}\left(1^{-}, 2^{-}, 3^{+}, 4^{+}\right)=\frac{i \kappa^{2}}{4}\left(\frac{\langle 12\rangle^{4}}{\langle 12\rangle\langle 23\rangle\langle 34\rangle\langle 41\rangle}\right)^{2} \times \frac{s t}{u}
$$

(This tree amplitude has been calculated previously in refs. [64,60] and was given in this helicity basis form by Berends, Giele and Kuijf in ref. [20].)

The amplitudes for the supersymmetric multiplets given in table 5.2 are

$$
\begin{aligned}
& A^{N=1}=\frac{F\left(t^{2}+14 t u+u^{2}\right)}{24 s^{4}}-\frac{F t^{2} u^{2}\left(\ln ^{2}(-t /-u)+\pi^{2}\right)}{2 s^{6}} \\
& \quad \quad \quad \frac{F(t-u)\left(t^{2}+8 t u+u^{2}\right) \ln (-t /-u)}{12 s^{5}} \\
& A^{N=4}=\frac{F}{2 s^{4}}\left((t-u) s \ln (-t /-u)-t u\left(\ln ^{2}(-t /-u)+\pi^{2}\right)+s^{2}\right) \\
& A^{N=6}=\frac{-F}{2}\left(\frac{\ln ^{2}(-t /-u)+\pi^{2}}{s^{2}}\right) \\
& A^{N=8}=\frac{2 F}{\epsilon}\left(\frac{\ln (-u)}{s t}+\frac{\ln (-t)}{s u}+\frac{\ln (-s)}{t u}\right) \\
& \quad+2 F\left(\frac{\ln (-t) \ln (-s)}{s t}+\frac{\ln (-u) \ln (-t)}{t u}+\frac{\ln (-s) \ln (-u)}{u s}\right)
\end{aligned}
$$

We chose to express the amplitude in the (unphysical) regime where all momentum variables $s, t$ and $u$ are negative. One can obtain expressions in the physical region by the substitution

$$
\ln (-x) \rightarrow \ln (|x|)-i \pi \Theta(x)
$$

$\Theta(s)$ is the Heavyside function where $\Theta(x)=1, x>0$ and $\Theta(x)=0, x<0$.

The pure gravity amplitude can be found from (5.52) using the expression in equ. (V.1), 
which gives the result

$$
\begin{aligned}
& A^{[2]}\left(1^{-}, 2^{-}, 3^{+}, 4^{+}\right) \\
&=F\left(\frac{2}{\epsilon}\left(\frac{\ln (-u)}{s t}+\frac{\ln (-t)}{s u}+\frac{\ln (-s)}{t u}\right)\right. \\
&+\frac{2 \ln (-u) \ln (-s)}{s u}+\frac{2 \ln (-t) \ln (-u)}{t u}+\frac{2 \ln (-t) \ln (-s)}{t s} \\
&+\frac{\left(4 t^{6}+14 u t^{5}+28 u^{2} t^{4}+35 t^{3} u^{3}+28 u^{4} t^{2}+14 u^{5} t+4 u^{6}\right)}{s^{8}}\left(\ln ^{2}(-t /-u)+\pi^{2}\right) \\
&+\frac{(t-u)\left(261 t^{4}+809 t^{3} u+1126 t^{2} u^{2}+809 t u^{3}+261 u^{4}\right)}{30 s^{7}} \ln (-t /-u) \\
&\left.+\frac{1682 t^{4}+5303 t^{3} u+7422 t^{2} u^{2}+5303 t u^{3}+1682 u^{4}}{180 s^{6}}\right)
\end{aligned}
$$

It is clear from (5.52) and (5.54) that only amplitudes containing gravitons have divergent components. In chapter 3 , we showed that amplitudes in which the only external particles are gravitons should be UV finite. If this is true then the, divergences seen here should be purely IR. We will confirm this in chapter 7 .

We have shown how we can use a closed bosonic string to construct a set of rules for the efficient calculation of one-loop graviton scattering amplitudes. These rules are applicable to any combination of particles in the loop and any number of external gravitons. We find that the method constitutes a powerful alternative to the conventional techniques and, as a result, allows us to carry out many calculations which have not been practical up to now.

Using this string-inspired method, we were able to produce a complete set of fourgraviton one-loop amplitudes. In order to confirm the validity of these results, it would be useful to have independent checks. There are a few we can consider. First, it is clear that these amplitudes exhibit the correct symmetries. Second, we can confirm that our result for internal scalars agrees with the one found by Grisaru and Zak [64]. Finally, we can consider the information provided by the Cutkosky rules. This will be the focus of the next chapter. 
Jolly agreed, so the toys set to work. Little Bear cut ... and Rabbit fixed them in place with glue and pins. Jane Hissey in 'Jolly Tall'

\section{Cutkosky Rules}

The Cutkosky rules $[5,16,1]$ allow us to find the discontinuities of an amplitude. If we can take these discontinuities and relate them in a unique way to other terms which can appear in an amplitude, then the rules will allow us to constrain or reconstruct the form of the amplitude to some extent. We will use these ideas to obtain parts of amplitudes we are interested in. Some we can construct completely; for the others we find parts which provide a check on the string calculations.

\subsection{Introduction}

We can formulate the rules in the following way (see, e.g., [1]): Consider 'cutting' a Feynman diagram into two separate parts (e.g", as in fig. 6.A). Then we can find the discontinuities in channel $x$ associated with that cut.

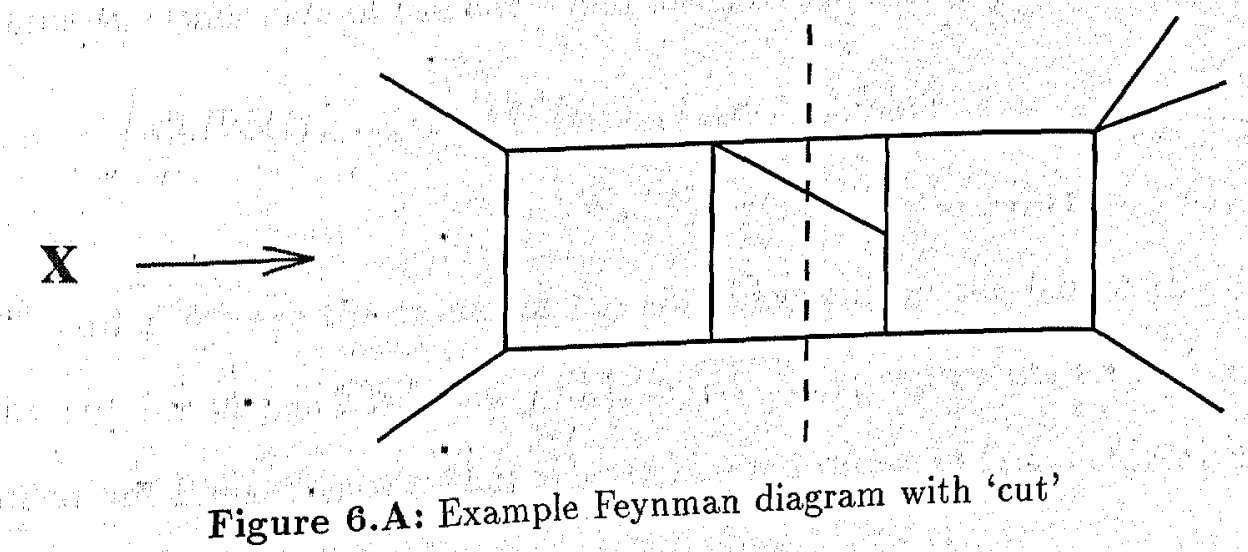

The normal Feynman rules should be used, except that all legs which are 'on the cut' should be taken to be on-shell - where we would use their propagator in the calculation we insert $\sim \theta(k) \delta\left(k^{2}-m^{2}\right.$ ) instead. (Here, $k$ and $m$ are the momentum and mass of the 
propagating particle respectively.) This gives us an equation for the discontinuous parts of this amplitude:

$$
\begin{array}{r}
I_{\text {discont }} \sim \int \prod_{i=1}^{m} \frac{d^{4} q_{i}}{(2 \pi)^{4}} \prod_{j=1}^{n} V_{j} \prod_{k=1}^{m_{1}} \frac{1}{q_{k}^{2}-m_{l}^{2}} \prod_{k=m_{1}+1}^{m_{2}} \frac{1}{q_{k}^{2}-m_{l}^{2}} \\
\times \prod_{r=m_{2}+1}^{m}(2 \pi)^{4} \theta\left(q_{r}^{0}\right) \delta\left(q_{r}^{2}-m_{r}^{2}\right)
\end{array}
$$

when the (original, uncut) diagram has $n$ vertices $V_{i}$ and $m$ propagators. $q_{1} \ldots q_{m_{1}}$ and $q_{m_{1}+1} \ldots q_{m_{2}}$ are the momenta of propagators on the left and right sides of the cut respectively; the remaining are those which cross the cut.
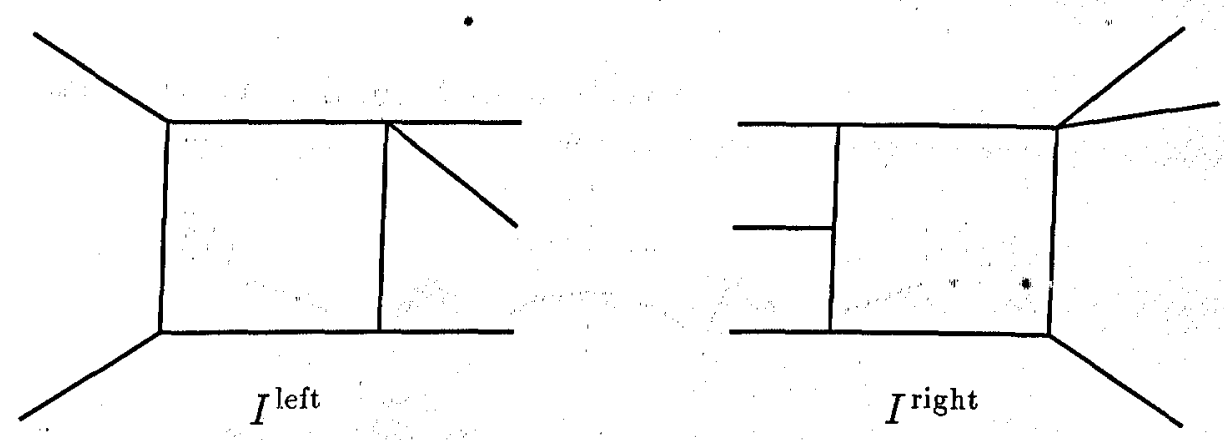

Figure 6.B: The sub-diagrams on either side of the cut in fig. 6.A.

It is easy to see that this expression splits into two distinct parts - one for each of the sub-diagrams on either side of the cut - and leads to the integral

$$
\begin{array}{r}
I_{\text {discont }} \sim \int d \operatorname{LIPS}\left(\ell_{1}, \ldots, \ell_{n}\right) I^{\text {left }}\left(\ell_{1}, \ldots \ell_{n}, q_{1}, \ldots, q_{m_{1}}\right) \\
\quad \times I^{\text {right }}\left(-\ell_{1}, \ldots,-\ell_{n}, q_{m_{1}+1}, \ldots, q_{m_{2}}\right) .
\end{array}
$$

Where $I^{\text {left }}$ and $I^{\text {right }}$ are the values of the sub-diagrams on the left of the cut and the right of the cut (as in fig. 6.B.) and $\int d \operatorname{LIPS}\left(\ell_{1}, \ldots, \ell_{n}\right)$ represents an integral over the 'Lorentz Invariant Phase Space'. That is, over allowed values of $\ell_{1}, \ldots, \ell_{n}$, with the added constraint that $\ell_{i}^{2}=m_{i}^{2}$ for every $i$.

We can use this method in precisely the same way with full amplitudes as we do with Feynman diagrams $[17,10]$. For instance, we can find the discontinuities of a one-loop amplitude by sewing together pairs of on-shell trees. This is obviously a more efficient 
application of the rules - the full amplitudes will generally be simpler than the contributions from individual diagrams (in particular, many sets of diagrams sum to give vanishing amplitudes) and we are only required to carry out a small number of calculations. This has been used successfully to calculate a wide range of one-loop amplitudes in QCD $[17,10]$.

It is this 'amplitude level' option which we implement here for gravity. As well as being a more efficient application, we will find that this will enable us to constrain the amplitudes more tightly. In fact, in some cases we can, produce a 'uniqueness theorem' for our results. That is, a theorem which allows us to identify those results which are determined uniquely by their cuts.

\subsection{Application to One-Loop Calculations}

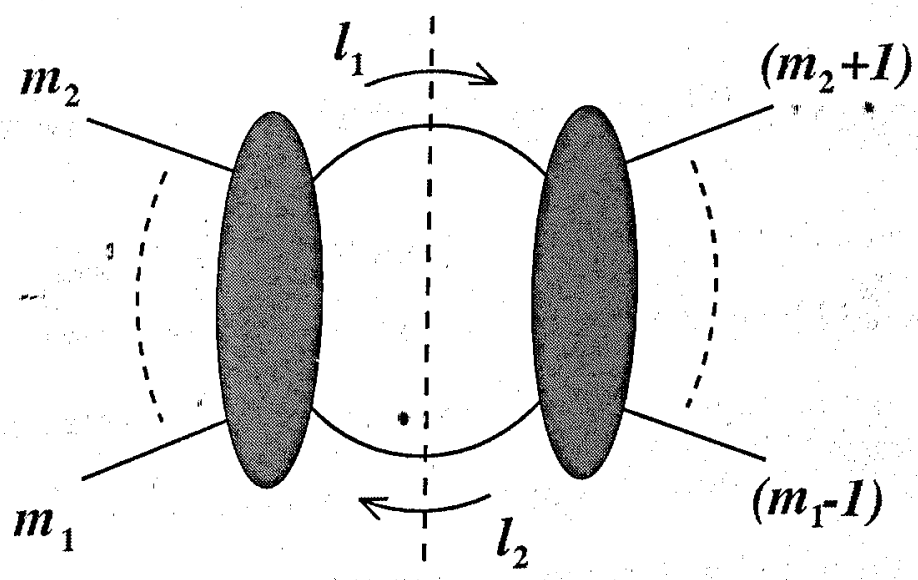

Figure 6.C: Generic cut in a one-loop amplitude.

Now we look in more detail at the way these rules are applied to calculations. At this stage we concentrate on one-loop amplitudes with external gravitons. We also restrict ourselves to massless particles. In the cases we are looking at here - simple physical amplitudes the discontinuous parts are all products of terms

$$
i \theta(x)
$$

$\theta(x)$ is defined to be 0 if $x<0$ and 1 if $x>0 . x$ is one of the momentum invariants of the amplitude. For simplicity, and without loss of information, we consider one channel of the 
amplitude at a time. We can regard this as taking the momentum invariants corresponding to all other channels to be negative so that their discontinuous parts vanish. We are then looking for terms precisely like (6.3).

Consider doing this for the amplitude and channel given in fig. 6.C. The Cutkosky rules for this give

$$
\frac{i}{2} \int d \operatorname{LIPS}\left(-\ell_{1}, \ell_{2}\right) A^{\text {tree }}\left(-\ell_{1}, m_{1}, \ldots, m_{2}, \ell_{2}\right) A^{\text {tree }}\left(-\ell_{2}, m_{2}+1, \ldots, m_{1}-1, \ell_{1}\right) .
$$

As we have indicated, the $\int d \operatorname{LIPS}\left(-\ell_{1}, \ell_{2}\right)$ is the integral over all allowed values of $\ell_{1}$ and $\ell_{2}$ with $\ell_{1}^{2}=\ell_{2}^{2}=0$. Rather than calculate this integral directly, we relate it to another which we know has the same cuts. This enables to us identify more clearly which terms are linked with the discontinuities. The simplest choice is the off-shell scalar integral

$$
-\left.\int \frac{d^{4} \ell_{1}}{(2 \pi)^{4}} A^{\text {tree }}\left(-\ell_{1}, m_{1}, \ldots, m_{2}, \ell_{2}\right) \frac{1}{\ell_{2}^{2}} A^{\text {tree }}\left(-\ell_{2}, m_{2}+1, \ldots, m_{1}-1, \ell_{1}\right) \frac{1}{\ell_{1}^{2}}\right|_{\text {cuts }}
$$

(From the above discussion of Cutkosky rules, it is clear that this leads to (6.4)). So, we can calculate this integral and extract the terms which we would have found from (6.4).

During the calculation we may use $\ell_{1}^{2}=\ell_{2}^{2}=0$ in the numerator at any point since these terms cancel a propagator and the result will not produce a discontinuous factor. (If this were not the case, then we would find an inconsistency between (6.4) and (6.5).)

This calculation allows us to find terms of the form (6.3) with $x=\left(m_{1}+\cdots+m_{2}\right)^{2}$. We can immediately relate these to terms

$$
\ln (-x)=\ln (|x|)-i \pi \theta(x)
$$

It may also be possible to extend these relations to other terms, but in many cases ambiguities restrict this.

Since we require all the discontinuities in a channel, we must sum over contributions for all relevant particles in the loop. The result will give us a large part of the amplitude, at which point we can deduce how much, if anything, is still undetermined in our results. 


\subsection{Uniqueness}

In order to determine how much of the complete amplitude we have constructed we can look to the 'uniqueness theorem' [17]. This result states that

An $m$-point 1-loop amplitude can be determined completely from its cuts (it is 'cut-constructible') if its integral has at most $m-2$ powers of the loop momentum in the numerator of the integrand.

(The exception is the two-point amplitude which is cut-constructible if the integrand has at most one power of loop momentum in the numerator.) The proof can be summarised as follows: All $m$-point tensor integrals in $4-2 \epsilon$ dimensions can be reduced (by, for instance, Passarino-Veltman [85] techniques) to a sum of scalar box, tensor triangle and tensor bubble integrals. If we restrict ourselves to integrals which obey the condition in the theorem then we can make this more precise: all $m$-point tensor integrals reduce to a sum of scalar box and linear triangle and bubble integrals. If we define $\mathcal{F}_{n}$ to be the set of functions relating to these integrals then the cuts of an amplitude, $A_{n}$, can be described as

$$
A_{n}=\left.\sum_{i \mid I_{i} \in \mathcal{F}_{n}} c_{i} I_{i}\right|_{\text {cuts }}
$$

If there is another combination

$$
A_{n}=\left.\sum_{i \mid I_{i} \in \mathcal{F}_{n}} c_{i}^{\prime} I_{i}\right|_{\text {cuts }}
$$

then we know that

$$
\begin{aligned}
&\left.\sum_{i \mid I_{i} \in \mathcal{F}_{n}}\left(c_{i}-c_{i}^{\prime}\right) I_{i}\right|_{\text {cuts }}=0 \\
& \Longrightarrow \sum_{i \mid I_{i} \in \mathcal{F}_{n}}\left(c_{i}-c_{i}^{\prime}\right) I_{i}= \\
&=\text { rational function } \\
& \text { of momentum invariants }
\end{aligned}
$$


By looking at the places in $\mathcal{F}_{n}$ where logarithms and dilogarithms are found and the polynomials associated with these it is possible to show that the right hand side of the last equation vanishes. So,

$$
A_{n}=\sum_{i \mid I_{i} \in \mathcal{F}_{n}} c_{i} I_{i}
$$

To see this explicitly, let us restrict ourselves to calculations with four external parti-* cles. In this case all integral functions required to construct an amplitude can be written as linear combinations of only ten. These are the scalar box, $I_{4}(x, y)$; triangles, $I_{3}(x)$; and bubble, $I_{2}(x)$, integrals, plus a linear combination of bubbles, $J_{2}$ :

$$
\begin{aligned}
I_{4}(x, y) & =r_{\Gamma} \frac{1}{x y}\left\{\frac{2}{\epsilon^{2}}\left[(-x)^{-\epsilon}+(-y)^{-\epsilon}\right]-\ln ^{2}\left(\frac{-x}{-y}\right)-\pi^{2}\right\}, \\
& =r_{\Gamma} \frac{1}{x y}\left\{\frac{4}{\epsilon^{2}}-\frac{2 \ln (-x)+2 \ln (-y)}{\epsilon}+2 \ln (-x) \ln (-y)-\pi^{2}\right\} \\
I_{3}(x) & =\frac{r_{\Gamma}}{\epsilon^{2}}(-x)^{-1-\epsilon}=-\frac{r_{\Gamma}}{x}\left(\frac{1}{\epsilon^{2}}-\frac{\ln (-x)}{\epsilon}+\frac{\ln ^{2}(-x)}{2}\right) \\
I_{2}(x) & =\frac{r_{\Gamma}}{\epsilon(1-2 \epsilon)}(-x)^{-\epsilon}=r_{\Gamma}\left(\frac{1}{\epsilon}-\ln (-x)+2\right) \\
J_{2} & =r_{\Gamma}
\end{aligned}
$$

with $x, y \in\{s, t, u\}$. (This produces a total of ten when we consider all possible choices for $x$ and $y$.)

Nearly all the expressions in (6.11) are identifiable by one logarithmic term which is unique to that function at $\mathcal{O}(\epsilon)$. The exception is the last. It is this which will cause problems in general - it allows the possibility of polynomial terms which cannot be identified by cut results. Clearly, if we could discard this we would be able to find amplitudes with no ambiguities when we know their cuts. This is precisely what we can do for the 'cut-constructible' cases.

Within the set of amplitudes we are looking at here, the number which fill the criterion are small since a general 1-loop (non-supersymmetric) gravity amplitude has $2 n$ powers of loop momentum. If there are cut-constructible amplitudes they will be the ones for SUSY multiplets since the degree of the loop momentum polynomial gets smaller with 
increasing $N$. In fact, $N=8$ and $N=6$ multiplets have a reduction of 8 and 6 powers of loop momentum respectively. For the $N=8$ case this means that an amplitude is cut-constructible if $2 n-8 \leq n-2$, implying that we will be able to obtain the 4 and 5 graviton amplitudes completely. For $N=6$ multiplets only the 4 graviton amplitude will be obtainable in its entirety.

\subsection{External Graviton Calculations}

As an example of this method let us look at some of the amplitudes found using the string-based rules.

First note a trivial result: Consider the amplitudes $A(+,+,+,+)$ and $A(-,+,+,+)$. The cut diagrams for these amplitudes must be of the form in fig. 6.D. (Since we take all particles to be incoming the helicity of a particle in the loop flips as we cross the cut.) It is impossible to choose particles on the internal lines such that both trees are nonvanishing (see appendix IV and ref. [28]); our integral is trivially zero and we deduce that the amplitudes should have no logarithms. This is consistent with our string calculations for these helicity choices.

These cases give a simple example of the uniqueness condition: The cuts are zero in all cases. In particular, they are zero for the $N=8$ and $N=6$ multiplets. Using the result in the previous section we deduce that the whole amplitude should be zero. This is confirmed by both the SUSY identities and the string calculations.

The cuts of $\dot{A}\left(1^{-}, 2^{-}, 3^{+}, 4^{+}\right)$are more complex. Let us begin with those in the s-channel. In this case equation (6.4) becomes

$$
\frac{i}{2} \sum_{\substack{\text { internal } \\ \text { particles }}} \int d \operatorname{LIPS} A^{\text {tree }}\left(1^{-}, 2^{-}, \ell_{2}^{+}, \ell_{1}^{+}\right) A^{\text {tree }}\left(\ell_{1}^{-}, \ell_{2}^{-}, 3^{+}, 4^{+}\right)
$$

The only internal particles which give a non-vanishing result are gravitons. Fermion contributions vanish because their helicity is not flipped by the graviton vertex, implying that $A\left(g, g, \psi^{+}, \psi^{+}\right)=0$. The same holds for vector and scalar amplitudes at tree level (taking 


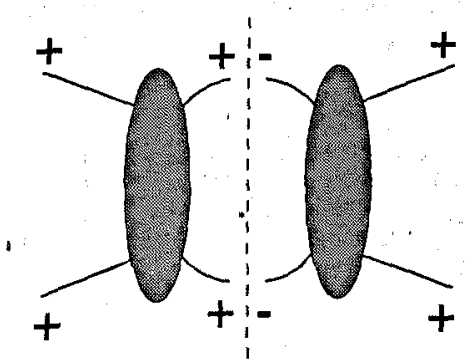

(a)
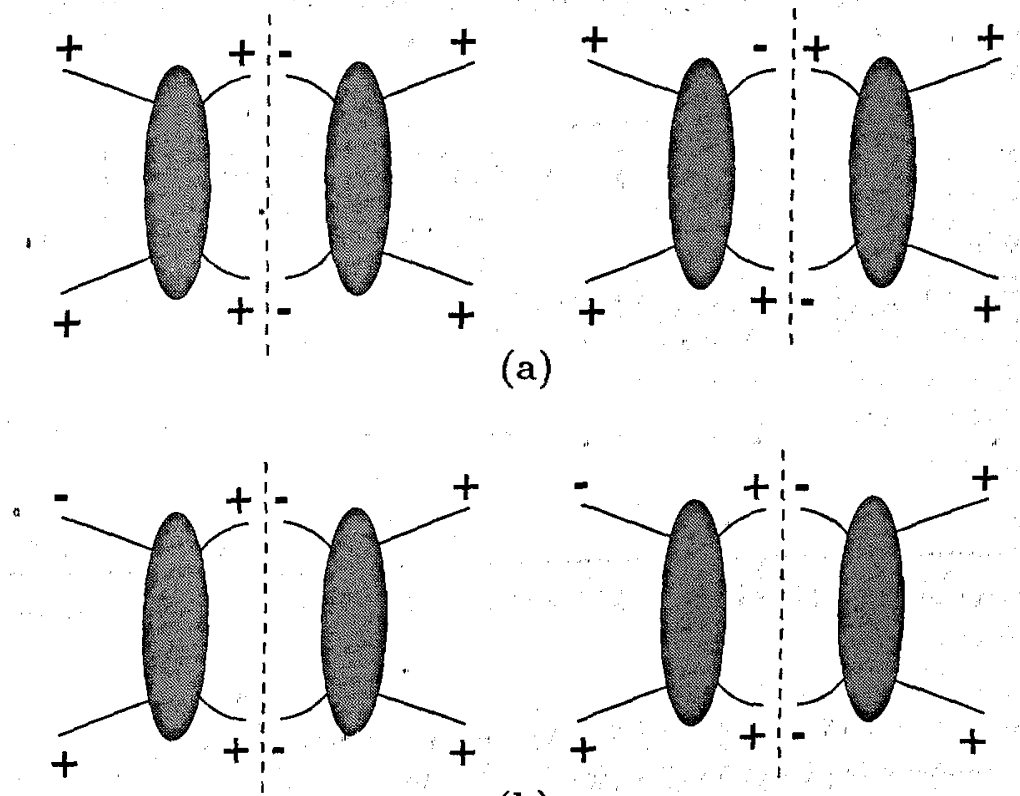

(b)

Figure 6.D: Possible helicity configurations in the cut calculations of (a) $A(+,+,+,+)$ and (b) $A(-,+,+,+)$. In all cases the tree on the right-hand side vanishes.

particle/antiparticle instead of positive/negative helicity in the latter) [86]. These results can be deduced from SUSY relations (see appendix IV). This implies that the only place logarithmic terms can appear is in amplitudes with gravitons in the loop. Let us look in detail at this case - the simplest non-trivial example of applying cut rules - depicted in fig. 6.E.

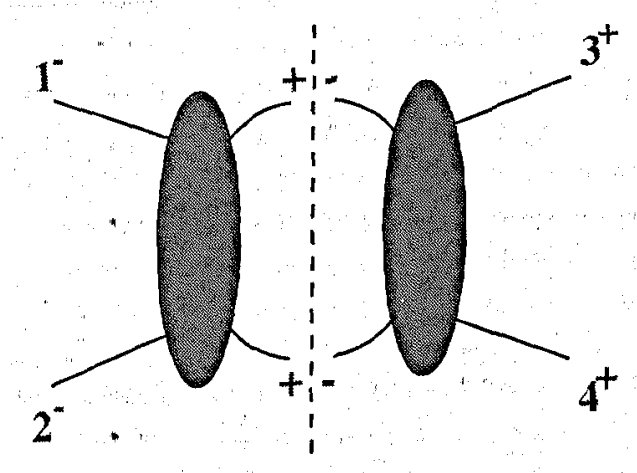

Figure 6.E: Helicity configuration for the $A\left(1^{-}, 2^{-}, 3^{+}, 4^{+}\right) s$-channel cut

The tree expression is the one defined in (5.51):

$$
A^{\text {tree }}\left(1^{-}, 2^{-}, 3^{+}, 4^{+}\right)=\frac{i \kappa^{2}}{4}\left(\frac{\langle 12\rangle^{4}}{\langle 12\rangle\langle 23\rangle\langle 34\rangle\langle 41\rangle}\right)^{2} \times \frac{s t}{u} \text {. }
$$


Inserting this in (6.12) we find the cut equation for this process

$$
\begin{array}{r}
-\frac{i \kappa^{4}}{32} \int d \operatorname{LIPS}\left(\frac{\langle 12\rangle^{4}}{\langle 12\rangle\left\langle 2 \ell_{2}\right\rangle\left\langle\ell_{2} \ell_{1}\right\rangle\left\langle\ell_{1} 1\right\rangle}\right)^{2} \frac{s\left(k_{2} \cdot \ell_{2}\right)}{\left(k_{1} \cdot \ell_{2}\right)} \\
\times\left(\frac{\left\langle\ell_{1} \ell_{2}\right\rangle^{4}}{\langle 34\rangle\left\langle 4 \ell_{1}\right\rangle\left\langle\ell_{1} \ell_{2}\right\rangle\left\langle\ell_{2} 3\right\rangle}\right)^{2} \frac{s\left(k_{3} \cdot \ell_{2}\right)}{\left(k_{4} \cdot \ell_{2}\right)}
\end{array}
$$

which we can rearrange to be,

$$
\begin{aligned}
= & \frac{i \kappa^{4}}{32} s^{2}\left(\frac{\langle 12\rangle^{4}}{\langle 12\rangle\langle 34\rangle}\right)^{2} \int d \operatorname{LIPS} \frac{\left(k_{2} \cdot \ell_{2}\right)}{\left(k_{1} \cdot \ell_{2}\right)} \frac{\left(k_{3} \cdot \ell_{2}\right)}{\left(k_{4} \cdot \ell_{2}\right)} \frac{\left\langle\ell_{1} \ell_{2}\right\rangle^{4}}{\left\langle 2 \ell_{2}\right\rangle^{2}\left\langle\ell_{1} 1\right\rangle^{2}\left\langle 4 \ell_{1}\right\rangle^{2}\left\langle\ell_{2} 3\right\rangle^{2}} \\
= & -\frac{\kappa^{2}}{8} A^{\text {tree }}\left(1^{-}, 2^{-}, 3^{+}, 4^{+}\right) s^{3} t u \int d \operatorname{LIPS} \frac{\left(k_{2} \cdot \ell_{2}\right)}{\left(k_{1} \cdot \ell_{2}\right)} \frac{\left(k_{3} \cdot \ell_{2}\right)}{\left(k_{4} \cdot \ell_{2}\right)} \\
& \times \frac{1}{16\left(k_{2} \cdot \ell_{2}\right)\left(k_{1} \cdot \ell_{1}\right)\left(k_{3} \cdot \ell_{2}\right)\left(k_{4} \cdot \ell_{1}\right)} \\
= & -\frac{\kappa^{2}}{8} A^{\text {tree }}\left(1^{-}, 2^{-}, 3^{+}, 4^{+}\right) s^{3} t u \int d \operatorname{LIPS} \frac{1}{16\left(k_{1} \cdot \ell_{2}\right)\left(k_{4} \cdot \ell_{2}\right)\left(k_{1} \cdot \ell_{1}\right)\left(k_{4} \cdot \ell_{1}\right)}
\end{aligned}
$$

Recall that, in the discussion of the uniqueness condition, we said that amplitudes could be reduced to scalar boxes plus tensor triangles and bubbles. In this case we can reduce it to four scalar boxes using the relation

$$
\begin{array}{r}
\frac{1}{\left(k_{1} \cdot \ell_{2}\right)\left(k_{4} \cdot \ell_{2}\right)\left(k_{1} \cdot \ell_{1}\right)\left(k_{4} \cdot \ell_{1}\right)}+\frac{1}{s^{2}}\left(\frac{-1}{\left(k_{1} \cdot \ell_{1}\right)\left(k_{4} \cdot \ell_{1}\right)}+\frac{1}{\left(k_{1} \cdot \ell_{2}\right)\left(k_{4} \cdot \ell_{1}\right)}\right. \\
=\frac{1}{\left.+\frac{1}{\left(k_{1} \cdot \ell_{1}\right)\left(k_{4} \cdot \ell_{2}\right)}+\frac{-1}{\left(k_{1} \cdot \ell_{2}\right)\left(k_{4} \cdot \ell_{2}\right)}\right)} \\
=\frac{16}{s^{2}}\left(\frac{1}{\left(k_{1}-\ell_{1}\right)^{2}\left(k_{4}+\ell_{1}\right)^{2}}+\frac{1}{\left(k_{1}+\ell_{2}\right)^{2}\left(k_{4}+\ell_{1}\right)^{2}}\right. \\
\left.+\frac{1}{\left(k_{1}-\ell_{1}\right)^{2}\left(k_{4}-\ell_{2}\right)^{2}}+\frac{1}{\left(k_{1}+\ell_{2}\right)^{2}\left(k_{4}-\ell_{2}\right)^{2}}\right)
\end{array}
$$

(This depends on the trees being on-shell.)

To see explicitly that this gives the sum of scalar boxes, we can carry out the substitutions which turn the $d$ LIPS integral to the off-shell integral as in (6.4) and (6.5). So, for 


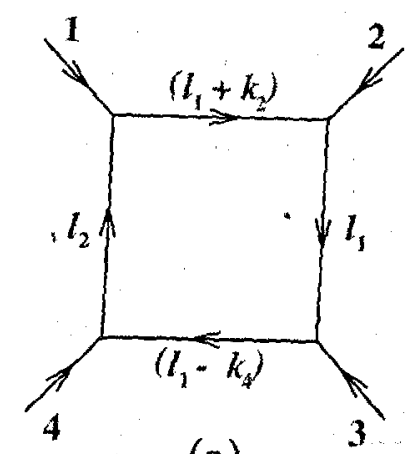

(a)

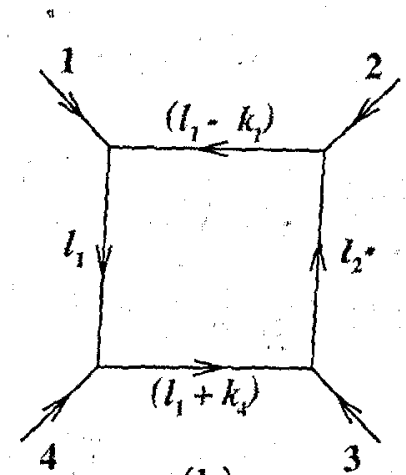

(b)

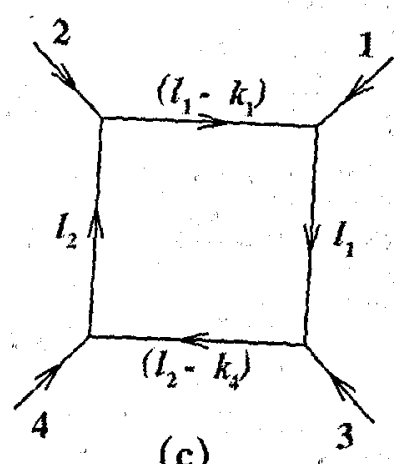

(c)

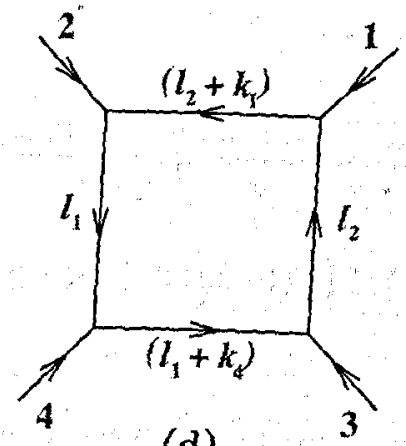

(d)

Figure 6.F: Boxes which contribute to the $A\left(1-, 2^{-}, 3^{+}, 4^{+}\right)$calculation.

Note that (a) is related to (b) and (c) to (d) by redefinition of loop momenta.

example, the first term in (6.16) leads to

$$
\begin{aligned}
\sim \int \frac{d^{4} l_{1}}{(2 \pi)^{4}} & \frac{1}{l_{1}^{2}\left(k_{1}-\ell_{1}\right)^{2} l_{2}^{2}\left(k_{4}+\ell_{1}\right)^{2}} \\
& =\int \frac{d^{4} l_{1}}{(2 \pi)^{4}} \frac{1}{l_{1}^{2}\left(\ell_{1}-k_{1}\right)^{2}\left(\ell_{1}-k_{1}-k_{2}\right)^{2}\left(l_{1}-k_{1}-k_{2}-k_{3}\right)^{2}}
\end{aligned}
$$

which is the box integral depicted in fig. 6.F(b). In this way, we can clearly see that (6.15) can be expressed as the sum of the boxes in fig. $6 . F$ with coefficients

$$
\frac{\kappa^{2}}{8} A^{\text {tree }}\left(1^{-}, 2^{-}, 3^{+}, 4^{+}\right) \text {stu. }
$$

We see in the figure that there are two independent boxes we must consider - those with ordering of external legs 1234 and 2134. The integral for these orderings evaluates to, respectively,

$$
\begin{aligned}
I_{4}^{1234} & =\frac{r_{\Gamma}(4 \pi)^{\epsilon}}{(4 \pi)^{2}} \frac{1}{s t}\left\{\frac{2}{\epsilon^{2}}\left[(-s)^{-\epsilon}+(-t)^{-\epsilon}\right]-\ln ^{2}(-s /-t)-\pi^{2}\right\} \\
& =\frac{r_{\Gamma}(4 \pi)^{\epsilon}}{(4 \pi)^{2}} \frac{1}{s t}\left\{\frac{4}{\epsilon^{2}}-\frac{2}{\epsilon}(\ln (-s)+\ln (-t))+2 \ln (-s) \ln (-t)-\pi^{2}\right\}
\end{aligned}
$$


and

$$
\begin{aligned}
I_{4}^{2134} & =\frac{r_{\Gamma}(4 \pi)^{\epsilon}}{(4 \pi)^{2}} \frac{1}{s t}\left\{\frac{2}{\epsilon^{2}}\left[(-s)^{-\epsilon}+(-u)^{-\epsilon}\right]-\ln ^{2}(-s /-u)-\pi^{2}\right\} \\
& =\frac{r_{\Gamma}(4 \pi)^{\epsilon}}{(4 \pi)^{2}} \frac{1}{s u}\left\{\frac{4}{\epsilon^{2}}-\frac{2}{\epsilon}(\ln (-s)+\ln (-u))+2 \ln (-s) \ln (-u)-\pi^{2}\right\}
\end{aligned}
$$

This allows us to deduce that the cuts in the $s$-channel are,

$$
\begin{aligned}
& \left.2 \frac{\kappa^{2}}{8} A^{\text {tree }}\left(1^{-}, 2^{-}, 3^{+}, 4^{+}\right) s t u\left(I^{1234}+I^{2134}\right)\right|_{\text {cuts }} \\
& =\frac{\kappa^{2}}{4} \frac{r_{\Gamma}(4 \pi)^{\epsilon^{\prime}}}{(4 \pi)^{2}} A^{\text {tree }}\left(1^{-}, 2^{-}, 3^{+}, 4^{+}\right) s t u \\
& \quad \times\left.\left(\frac{4}{\epsilon^{2}}\left(\frac{1}{s t}+\frac{1}{s u}\right)-\frac{2}{\epsilon} \ln (-s)\left(\frac{1}{s t}+\frac{1}{s u}\right)+\frac{2 \ln (-s)}{s}\left(\frac{\ln (-u)}{u}+\frac{\ln (-t)}{t}\right)\right)\right|_{\text {cuts }} \\
& =\frac{\kappa^{2}}{4} \frac{r_{\Gamma}(4 \pi)^{\epsilon}}{(4 \pi)^{2}} A^{\text {tree }}\left(1^{-}, 2^{-}, 3^{+}, 4^{+}\right) s t u\left(\frac{2}{\epsilon} s+2(t \ln (-u)+u \ln (-t))\right) \ln (-s)
\end{aligned}
$$

The $t$ - and $u$-channel cuts require essentially the same calculations since the helicity configurations are the same in both cases. We will calculate the $t$-channel case and deduce the $u$-channel result from this. In this case the possible helicity configurations of the trees are those in fig. 6.G. Since helicity is no longer flipped on either tree, the cuts do not vanish as before; we must include the contribution from all relevant particles across the cut. In theory, we could calculate the contribution from each particle in the loop individually by considering the cut with only that particle on the intermediate lines. (The structure of the trees discussed in appendix IV implies that only one particle type will be involved at a time.) However, these calculations are quite complex in practice and we find that it is more efficient to consider a supersymmetric decomposition of the amplitudes (appendix V). As with the string-based rules, calculations for SUSY multiplets in the loop prove to be simpler than those for individual particles. As before, we can reconstruct amplitudes for any other particle combinations from the SUSY results.

Since we are calculating for different particle types in the loop, we will require all tree amplitudes involving one pair of graviton and one pair of non-graviton particles; each pair will have one particle of each helicity. To obtain these expressions, observe that these can 


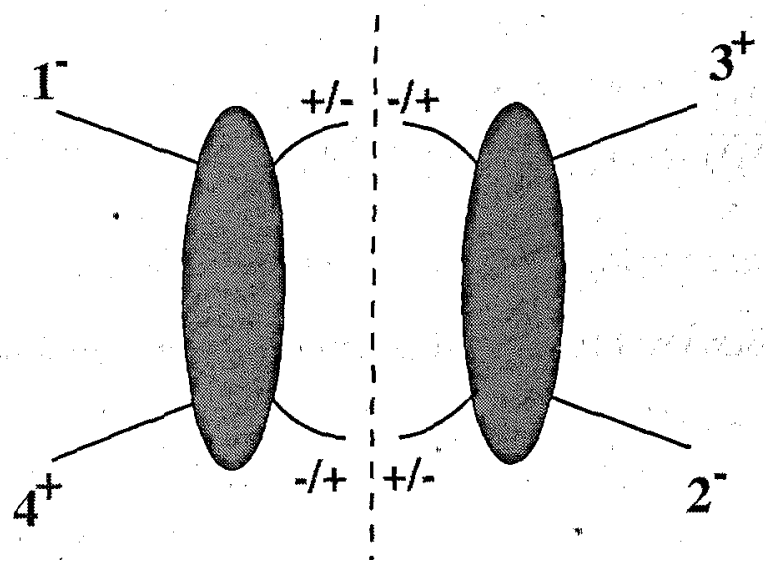

Figure 6.G: Helicity configurations for the $A\left(1^{-}, 2^{-}, 3^{+}, 4^{+}\right) t$-channel cut

all be related, via supersymmetric. Ward identities (See refs. $[22,23,2]$ and Appendix IV), to the two graviton/two scalar amplitude.

$$
\begin{aligned}
& A\left(g^{-}, g^{-}, g^{+}, g^{+}\right)=y^{4} A\left(g^{-}, \phi^{-}, \phi^{+}, g^{+}\right) \\
& A\left(g^{-}, \psi^{-}, \psi^{+}, g^{+}\right)=y^{3} A\left(g^{-}, \phi^{-}, \phi^{+}, g^{+}\right) \\
& A\left(g^{-}, A^{-}, A^{+}, g^{+}\right)=y^{2} A\left(g^{-}, \phi^{-}, \phi^{+}, g^{+}\right) \\
& A\left(g^{-}, \Lambda^{-}, \Lambda^{+}, g^{+}\right)=y A\left(g^{-}, \phi^{-}, \phi^{+}, g^{+}\right)
\end{aligned}
$$

Where $y=\langle 12\rangle /\langle 13\rangle$.

When we include all internal helicity configurations, cuts for the supersymmetric $N=n$ (matter) multiplet are then

$$
\begin{aligned}
& \frac{i}{2} \int d \operatorname{LIPS} A\left(4^{+}, 1^{-}, \phi\left(\ell_{2}\right)^{-}, \phi\left(\ell_{1}\right)^{+}\right) A\left(\phi\left(\ell_{1}\right)^{-}, \phi\left(\ell_{2}\right)^{+}, 2^{-}, 3^{+}\right) \rho_{n} \\
& \quad=-\frac{i \kappa^{4}}{32} \frac{t^{2}}{\langle 23\rangle^{2}\langle 41\rangle^{2}} \int d \operatorname{LIPS} \frac{\left\langle 1 \ell_{1}\right\rangle^{4}\left\langle 2 \ell_{1}\right\rangle^{4}\left\langle 1 \ell_{2}\right\rangle^{2}\left\langle 2 \ell_{2}\right\rangle^{2}}{\left\langle 3 \ell_{1}\right\rangle^{2}\left\langle 4 \ell_{1}\right\rangle^{2}\left\langle\ell_{1} \ell_{2}\right\rangle^{4}} \frac{\left(k_{1} \cdot \ell_{2}\right)\left(k_{2} \cdot \ell_{2}\right)}{\left(k_{1} \cdot \ell_{1}\right)\left(k_{2} \cdot \ell_{1}\right)} \rho_{n}
\end{aligned}
$$

where $\rho_{n}=\left(x-x^{-1}\right)^{n}$, with

$$
\therefore x^{2}=\frac{\left\langle 1 \ell_{2}\right\rangle\left\langle 2 \ell_{1}\right\rangle}{\left\langle 1 \ell_{1}\right\rangle\left\langle 2 \ell_{2}\right\rangle}
$$

We restrict ourselves to choices with even $n, n=2 m$. We can simplify $\rho_{n}$ as follows

$$
\begin{aligned}
\rho_{n}=\left(x-x^{-1}\right)^{2 m}=\frac{\left(x^{2}-1\right)^{2 m}}{x^{2 m}} & =\frac{\left(\left\langle 1 \ell_{2}\right\rangle\left\langle 2 \ell_{1}\right\rangle-\left\langle 1 \ell_{1}\right\rangle\left\langle 2 \ell_{2}\right\rangle\right)^{2 m}}{\left(\left\langle 1 \ell_{2}\right\rangle\left\langle 2 \ell_{1}\right\rangle\left(1 \ell_{1}\right\rangle\left\langle 2 \ell_{2}\right\rangle\right)^{m}} \\
& =\frac{\langle 12\rangle^{8}\left\langle\ell_{1} \ell_{2}\right\rangle^{2 m}}{\left(\left\langle 1 \ell_{2}\right\rangle\left\langle 2 \ell_{1}\right\rangle\left\langle 1 \ell_{1}\right\rangle\left\langle 2 \ell_{2}\right\rangle\right)^{m}} .
\end{aligned}
$$


So, the integral becomes

$$
-\frac{i \kappa^{4}}{32} t^{2} \frac{\langle 12\rangle^{2 m}}{\langle 23\rangle^{2}\langle 41\rangle^{2}} \int d \operatorname{LIPS} \frac{\left(k_{1} \cdot \ell_{2}\right)}{\left(k_{1} \cdot \ell_{1}\right)} \frac{\left(k_{2} \cdot \ell_{2}\right)}{\left(k_{2} \cdot \ell_{1}\right)} \frac{\left\langle\ell_{1} \ell_{2}\right\rangle^{2 m-4}}{\left(\left\langle\ell_{1} 4\right)\left\langle 3 \ell_{1}\right\rangle\right)^{2}} \frac{\left(\left\langle\ell_{2} 1\right\rangle\left\langle 2 \ell_{2}\right\rangle\right)^{4-m}}{\left(\left(\left\langle\ell_{2} 1\right\rangle\left\langle 2 \ell_{2}\right\rangle\right)^{m-2}\right.}
$$

The cases for the $N=8$ multiplet and the $N=6$ matter multiplet are the most interesting here since, for the four-point amplitude, they fulfil the conditions of the uniqueness theorem in the previous section. The $N=8(m=4)$ case is particularly simple; in this case, (6.26) becomes

$$
-\frac{i \kappa^{4}}{32} t^{2} \frac{\langle 12\rangle^{8}}{\langle 23\rangle^{2}\langle 41\rangle^{2}} \int d \operatorname{LIPS} \frac{\left(k_{1} \cdot \ell_{2}\right)}{\left(k_{1} \cdot \ell_{1}\right)} \frac{\left(k_{2} \cdot \ell_{2}\right)}{\left(k_{2} \cdot \ell_{1}\right)} \frac{\left\langle\ell_{1} \ell_{2}\right\rangle^{4}}{\left(\left\langle 1 \ell_{2}\right\rangle\left\langle\ell_{1} 4\right\rangle\left\langle\ell_{2} 2\right\rangle\left\langle 3 \ell_{1}\right\rangle\right)^{2}}
$$

which gives the cut to be

$$
\begin{aligned}
-\frac{i \kappa^{4}}{32} t^{4} s^{2}\left(\frac{\langle 12\rangle^{8}}{\langle 12\rangle^{2}\langle 23\rangle^{2}\langle 34\rangle^{2}\langle 41\rangle^{2}}\right) & 1 \\
& \times \int d \operatorname{LIPS} \frac{\left(k_{1} \cdot \ell_{2}\right)}{\left(k_{1} \cdot \ell_{1}\right)} \frac{\left(k_{2} \cdot \ell_{2}\right)}{\left(k_{2} \cdot \ell_{1}\right)} \frac{1}{16\left(k_{1} \cdot \ell_{2}\right)\left(k_{4} \cdot \ell_{1}\right)\left(k_{2} \cdot \ell_{2}\right)\left(k_{3} \cdot \ell_{1}\right)} .
\end{aligned}
$$

That is

$$
-\frac{\kappa^{2}}{8} A^{\text {tree }}\left(4^{+}, 1^{-}, 2^{-}, 3^{+}\right) t^{2} s \ddot{u} \int d \operatorname{LIPS} \frac{1}{16\left(k_{1} \cdot \ell_{1}\right)\left(k_{2} \cdot \ell_{1}\right)\left(k_{4} \cdot \ell_{1}\right)\left(k_{3} \cdot \ell_{1}\right)}
$$

As before, we can turn this into a sum of boxes. Giving a final result for the $N=8$ case of

$$
\begin{aligned}
A^{N=8}\left(1^{-}, 2^{-}, 3^{+}, 4^{+}\right) & =\kappa^{2} \frac{r_{\Gamma}(4 \pi)^{\epsilon}}{(4 \pi)^{2}} A^{\text {tree }}\left(1^{-}, 2^{-}, 3^{+}, 4^{+}\right) \\
\times & \left(\frac{2 s \ln (-s)+2 t \ln (-t)+2 u \ln (-u)}{\epsilon}\right. \\
+ & 2 s \ln (-t) \ln (-u)+2 t \ln (-u) \ln (-s)+2 u \ln (-s) \ln (-t))
\end{aligned}
$$

For the other choices of $N$ note that we can rearrange $\rho_{n}$ to give

$$
\rho_{n-2}=\frac{8 \operatorname{tr}_{+}\left(4 l_{1} 21 l_{1} 3\right)}{t s^{2}} \rho_{n}
$$

implying that

$$
\rho_{2 m}=\left(\frac{8 \operatorname{tr}_{+}\left(4 l_{1} 21 l_{1} 3\right)}{t s^{2}}\right)^{4-m} \rho_{8}
$$


We use the notation

$$
\operatorname{tr}\left(i l_{1} j m l_{1} n\right)=\operatorname{tr}\left(k_{i} l_{1} k_{j} k_{m} l_{1} k_{n}\right)
$$

and

$$
\operatorname{tr}_{ \pm}(\sigma)=\frac{1}{2} \operatorname{tr}\left(\left(1 \pm \gamma_{5}\right) \sigma\right)
$$

The integral for $N=2 m$ can then be written

$$
\begin{aligned}
-\frac{\kappa^{2}}{8} A^{\text {tree }}\left(4^{+}, 1^{-}, 2^{-}, 3^{+}\right) t^{2} s u \int d \operatorname{LIPS} \frac{1}{16\left(k_{1} \cdot \ell_{1}\right)\left(k_{2} \cdot \ell_{1}\right)\left(k_{4} \cdot \ell_{1}\right)\left(k_{3} \cdot \ell_{1}\right)} \\
\times\left(\frac{8 \operatorname{tr}_{+}\left(4 l_{1} 21 l_{1} 3\right)}{t s^{2}}\right)^{4-m}
\end{aligned}
$$

We can deal with this by first expanding the trace using commutation relations of $\gamma$ matrices $[1,17]$ to rewrite

$$
\operatorname{tr}_{+}\left(4 l_{1} 21 l_{1} 3\right)=\left(k_{1} \cdot l_{1}\right) \operatorname{tr}+\left(42 l_{1} 3\right)-\left(k_{2} \cdot l_{1}\right) \operatorname{tr}_{+}\left(41 l_{1} 3\right)
$$

We can also express the traces over four momenta as $[10,1]$

$$
\begin{aligned}
& \operatorname{tr}_{+}\left(42 l_{1} 3\right)=\frac{1}{2} \operatorname{tr}\left(42 l_{1} 3\right)+\frac{1}{2} \epsilon\left(42 l_{1} 3\right) \\
& \operatorname{tr}_{+}\left(41 l_{1} 3\right)=\frac{1}{2} \operatorname{tr}\left(41 l_{1} 3\right)+\frac{1}{2} \epsilon\left(41 l_{1} 3\right),
\end{aligned}
$$

where $\epsilon(i j k l)$ is defined by

$$
\epsilon(i j k l)=-4 i \epsilon^{\mu \nu \rho \sigma} k_{i \mu} k_{j} k_{m \rho} k_{n \sigma}
$$

We can deal with terms containing $\epsilon$ 's by using the substitutions for $\epsilon(i j k l)^{2}$ in appendix VI and the observation that

$$
\int d \operatorname{LIPS} \epsilon\left(42 l_{1} 3\right) f\left(1,2,3,4, l_{1}\right)=0 \int d \operatorname{LIPS} \epsilon\left(41 l_{1} 3\right) f\left(1,2,3,4, l_{1}\right)=0
$$

if $f$ contains only dot-products of the momenta (ie it has no $\epsilon$-tenors), so we can ignore any terms containing odd powers of $\epsilon(i j k l)$. We can further simplify the resulting expression in the same way as previous cut calculations. We are left with a (relatively) simple sum of 
boxes, triangles and bubbles. In all cases the cuts confirm the results found in the string calculations (up to finite polynomials in the momentum invariants).

In this chapter we have been able to use the constraints identified by the Cutkosky rules to reconstruct four-graviton amplitudes, confirming the results found by the string-inspired techniques. In most cases it has not been possible to find complete amplitudes by this method - there is always some ambiguity due to polynomial terms which are not identified by the cuts. We might wonder if we can overcome this problem and find unambiguous information for a wide range of amplitudes. This is the issue which we will address in chapters which follow. 


\section{Interlude: Analysis of Infinities}

At this point, let us make a short detour from the main work to look at the structure of infinities in the amplitudes we are calculating. The divergent parts of scattering amplitudes can be split into two distinct parts - the ultraviolet and infrared - which come from the divergences due to high and low momentum limits respectively. In general we are only interested in the ultraviolet contributions, since they are the one which determine whether a theory is renormalisable or not. (IR divergences are known to cancel in the S-matrix [67].) It will, therefore, be useful to have some way to distinguish the two contributions in our calculations. This will allow us to confirm that our result for the pure gravity amplitude is UV finite, as implied by our theoretical discussions. It will also be important in the next chapter, where we will look explicitly for UV divergences.

In many calculational techniques it is easy to tell the IR and UV contributions apart. However, in the Cutkosky rules there is some ambiguity in the source of infinities. It might be expected that we could separate the ultraviolet divergences from the infrared ones by looking at the limit in which internal momenta become large, as would be the case in Feynman diagram calculations. We can easily show that this is not a legitimate step in a cut calculation with a simple example: We know that we can add or subtract multiples of $\ell_{2}^{2}$ and $\ell_{1}^{2}$ to the numerator in a one-loop cut calculation without affecting the result (since the internal lines are on-shell). Consider adding $\ell_{1}^{2}$ to the numerator in a triangle calculation, for example the one depicted in fig. 7.A. This will give an extra term

$$
\frac{i}{2} \int d^{4} \ell_{2} \frac{\ell_{1}^{2}}{\ell_{1}^{2}\left(k_{3}+\ell_{1}\right)^{2} \ell_{2}^{2}}
$$




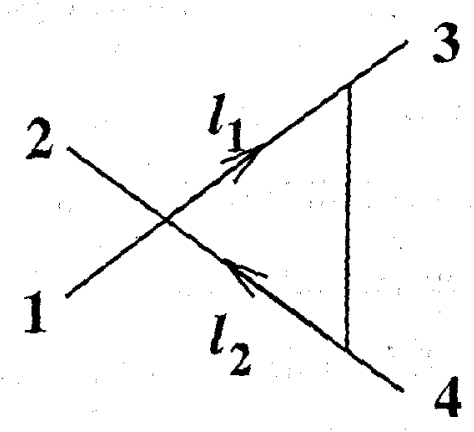

Figure 7.A: An example triangle calculation.

We know that $\ell_{1}=k_{1}+k_{2}+\ell_{2}$. So, we can rewrite $\ell_{1}^{2}$ as

$$
\begin{aligned}
\ell_{1}^{2}= & \left(k_{1}+k_{2}+\ell_{2}\right)^{2} \\
= & \left(s+2 k_{1} \cdot \ell_{2}+2 k_{2} \cdot \ell_{2}\right)^{2} \\
= & \left(2 k_{2} \cdot \ell_{2}\right)\left(2 k_{1} \cdot \ell_{2}\right)+s\left(2 k_{2} \cdot \ell_{2}\right)+s\left(2 k_{1} \cdot \ell_{2}\right) \\
& \quad+s^{2}+\left(2 k_{1} \cdot \ell_{2}\right)^{2}+\left(2 k_{2} \cdot \ell_{2}\right)^{2}
\end{aligned}
$$

If we took the approach of looking at the $\ell_{2}^{2} \rightarrow \infty$ limit for UV divergences, then adding (7.2) would imply an extra ultraviolet divergence (from the first term) of

$$
\sim \frac{s}{\epsilon}
$$

Since the overall effect of $\ell_{1}^{2}$ is zero, this divergence is cancelled by an additional apparent infrared infinity. So, adding $\ell_{1}^{2}$ makes an apparent shift between ultraviolet and infrared divergences. Clearly this implies that the source of infinities in cut calculations is ambiguous; while we can identify the divergences of amplitudes by this method, we cannot use it to separate them into ultraviolet and infrared contributions.

For our results to be useful, we must be able to identify or calculate one of the two infinite sectors. It is, in fact relatively simple to obtain IR divergences by another method, namely by looking at the soft limits of the amplitudes. That is, the limits obtained when the momentum of internal propagators goes to zero. 


\subsection{Infrared divergences/Soft Limits ${ }^{\dagger}$}

In order to find the infrared limits of an amplitude we must look at the effect of letting internal momenta tend to zero - so-called 'soft' limits. (In general, we must also consider the effect of two external particles become collinear; in the cases considered here these do not contribute.) In fact, we need only consider the zero momentum limit of those propagators on the 'edges of the diagram'. By this we mean those for which both ends meet an external line at some (3-point) vertex.

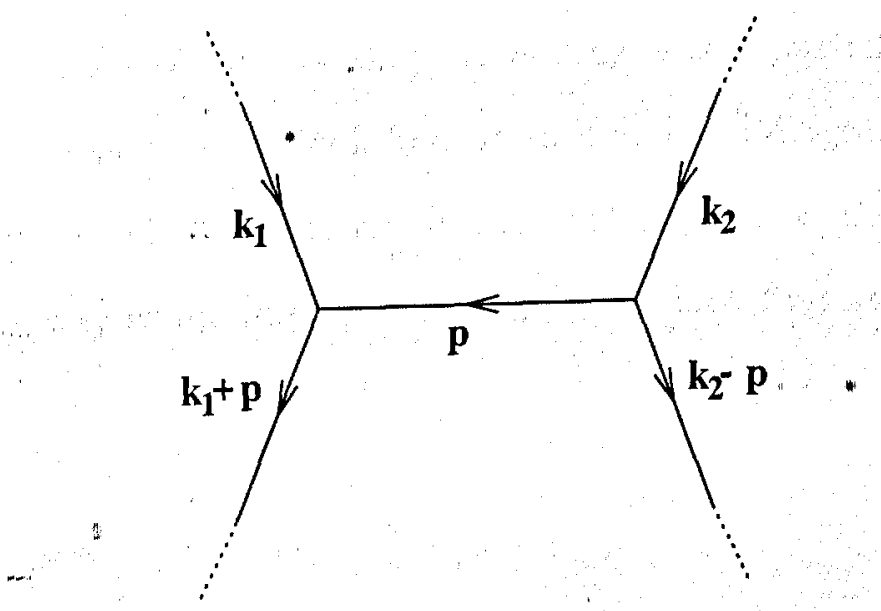

Figure 7.B: A sub-diaggram including a soft graviton.

To see this, consider the sub-diagram in fig. 7.B. As $p \rightarrow 0$ the dominant contribution from the $p$ integral will be

$$
\begin{aligned}
& \int \frac{d^{4-2 \epsilon} p}{\left(p+k_{1}\right)^{2} p^{2}\left(k_{2}-p\right)^{2}} \\
& \sim \int \frac{d^{4-2 \epsilon} p}{\left(k_{1}^{2}+2 k_{1} \cdot p\right) p^{2}\left(k_{2}^{2}-2 k_{2} \cdot p\right)}
\end{aligned}
$$

If either $k_{1}$ or $k_{2}$ (or both) are off-shell then this integral will not be infrared divergent. So, a propagator not on the edge of the diagram will not produce a contribution to the infrared divergences of an amplitude. On the other hand, if both $k_{1}$ and $k_{2}$ are external

† In this section we follow ref. [40] closely. The major difference in our analysis here is the use of dimensional regularisation. 
on-shell lines, then the integral will be

$$
\begin{aligned}
& \sim \int \frac{d^{4-2 \epsilon} p}{k_{1} \cdot p p^{2} k_{2} \cdot p} \\
& \sim \int \frac{d^{4-2 \epsilon} p}{|p|^{4}}
\end{aligned}
$$

Which produces a logarithmic infrared divergence.

Having established that only outermost propagators are relevant, it is clear that the simplest way to find the soft limits of a particular amplitude is to start with the amplitude with one less loop and look at the effect of adding a soft particle between two of the external lines. As an example, we"look first at an individual diagram rather than a whole amplitude. (It will be seen that the result does not depend on the diagram and can be applied in the same way at an amplitude level.) Let us look first at a diagram containing only gravitons.

\section{Gravitons}

Consider adding a soft graviton to an $(n-1)$-loop graviton diagram. To do this in practice, we connect two of the external lines with a virtual graviton and let the momentum of this particle (say $p$ ) tend to zero. If we attach such a particle line between legs with momentum $k_{1}$ and $k_{2}$, then we must add a propagator for the graviton, two three point vertices and two extra propagators with momenta $k_{1}+p$ and $k_{2}-p$ to the normal Feynman diagram expression for the $(n-1)$-loop amplitude.

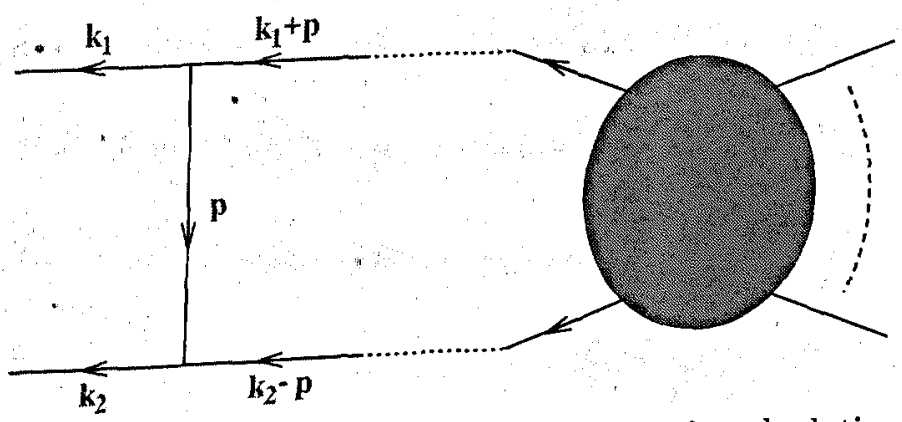

Figure 7.C: Representation of the soft limit calculation. 
Our construction can be pictured as the $n$-loop diagram in fig. 7.C. As $p \rightarrow 0$, we can express the integral for this as

$$
\frac{1}{(2 \pi)^{4-2 \epsilon}} \int \frac{d^{4-2 \epsilon} p B_{\alpha \beta \mu \nu}\left(k_{1}, k_{2}, p\right)}{\left(p+k_{1}\right)^{2} p^{2}\left(k_{2}-p\right)^{2}} A_{\alpha \beta \gamma \mu}^{(\mathrm{n}-1)-\text { loop }}
$$

Where

$$
\begin{aligned}
B_{\alpha \beta \gamma \mu}=\epsilon_{1 \alpha_{1}} \epsilon_{1 \beta_{1}} & \epsilon_{2 \mu_{1}} \epsilon_{2 \nu_{1}} V_{\alpha_{1} \beta_{1} \delta \gamma \alpha_{2} \beta_{2}}\left(k_{2},-p,-k_{2}+p\right) \\
\times & \frac{i}{2}\left(\delta_{\rho \delta} \delta_{\gamma \delta}+\delta_{\rho \gamma} \delta_{\delta \sigma}-\delta_{\gamma \delta} \delta_{\sigma \rho}\right) V_{\mu_{1} \nu_{2} \sigma \rho \mu_{2} \nu_{2}}\left(k_{1}, p,-k_{1}-p\right) \\
\times & \frac{i}{2}\left(\delta_{\mu_{2} \mu} \delta_{\nu_{2} \nu}+\delta_{\mu_{2} \nu} \delta_{\nu_{2} \mu}-\delta_{\mu_{2} \nu_{2}} \delta_{\mu \nu}\right) \\
& \times \frac{i}{2}\left(\delta_{\alpha_{2} \alpha} \delta_{\beta_{2} \beta}+\delta_{\alpha_{2} \beta} \delta_{\beta_{2} \alpha}-\delta_{\alpha_{2} \beta_{2}} \delta_{\alpha \beta}\right)
\end{aligned}
$$

(where $\mathrm{V}$ is the 3 -graviton vertex) and

$$
\epsilon_{1 \alpha} \epsilon_{1 \beta} \epsilon_{2 \gamma} \epsilon_{2 \mu} A_{\alpha \beta \mu \nu}^{(\mathrm{n}-1)-\mathrm{loop}}=\lim _{k_{1}^{2}, k_{2}^{2} \rightarrow 0} A^{(\mathrm{n}-1)-\mathrm{loop}}\left(k_{1}, k_{2}\right)
$$

$A^{(\mathrm{n}-1)-\mathrm{loop}}\left(k_{1}, k_{2}\right)$ is the $(n-1)$-loop amplitude for which the external legs with momenta $k_{1}$ and $k_{2}$ are off-shell. In general, the right hand side of (7.8) may not be equal to the on-shell $(n-1)$-loop amplitude. (See, for instance [11], which includes explicit examples of integral functions in which the limiting case does not equal the equivalent on-shell result.) Since we will only be considering trees here, this will not be a problem;

$$
\epsilon_{1 \alpha} \epsilon_{1 \beta} \epsilon_{2 \gamma} \epsilon_{2 \mu} A_{\alpha \beta \mu \nu}^{0-\text { loop }}=A^{\text {tree }}
$$

When we look at the $p \rightarrow 0$ limit of (7.7), we find that the dominant term is

$$
\begin{aligned}
B_{\alpha \beta \gamma \mu}\left(k_{1}, k_{2}\right) & =i \kappa^{2} \epsilon_{1 \alpha} \epsilon_{1 \beta} \epsilon_{2 \gamma} \epsilon_{2 \mu}\left(k_{1} \cdot k_{2}\right)^{2} \\
& =i \kappa^{2} \epsilon_{1 \alpha} \epsilon_{1 \beta} \epsilon_{2 \gamma} \epsilon_{2 \mu} \frac{\left(-2 k_{1} \cdot k_{2}\right)^{2}}{4}
\end{aligned}
$$

Note that we can discard all terms in which one or more momenta are contracted with the indices on $A^{(n-1)-\text { loop }}$ since this is known to be zero (see, e.g., appendix III). All $p$ dependent terms in the numerator can be disregarded since it would cause (7.5) to converge for small $p$. Hence, the only integral we need to look at is

$$
\frac{1}{(2 \pi)^{4-2 \epsilon}} \int \frac{d^{4-2 \epsilon} p}{\left(p+k_{1}\right)^{2} p^{2}\left(k_{2}-p\right)^{2}}
$$


This can be evaluated by usual Feynman parameter method. The result is,

$$
\frac{-i r_{\Gamma}}{(4 \pi)^{2-\epsilon}\left(-2 k_{1} \cdot k_{2}\right)^{1+\epsilon}}\left(\frac{1}{\epsilon^{2}}+\mathcal{O}\left(\epsilon^{0}\right)\right)
$$

Combining this with (7.10), we find that the divergence due to a soft graviton between external legs 1 and 2 for an n-loop graviton scattering diagram is given by

$$
\text { . } \quad \frac{r_{\Gamma} \kappa^{2}}{(4 \pi)^{2-\epsilon}} \frac{\left(-2 k_{1} \cdot k_{2}\right)^{1-\epsilon}}{4 \epsilon^{2}} \lim _{k_{1}^{2}, k_{2}^{2} \rightarrow 0} A^{(\mathrm{n}-1)-\operatorname{loop}}\left(k_{1}, k_{2}\right)
$$

In general, we require all the IR divergences for a diagram, rather than that due to a graviton connecting one pair of legs. To obtain this we simply sum over the above result for all pairs of adjacent external legs connected by a graviton line:

$$
\frac{r_{\Gamma} \kappa^{2}}{(4 \pi)^{2-\epsilon}} \frac{1}{4 \epsilon^{2}} \sum_{(i, j)}\left(-2 k_{i} \cdot k_{j}\right)^{1-\epsilon} \lim _{k_{1}^{2}, k_{2}^{2} \rightarrow 0} A^{(\mathrm{n}-1)-\operatorname{loop}}\left(k_{1}, k_{2}\right)
$$

As indicated above, we find that this result is not dependent on the form of $A^{(n-1)-\text { loop }}$. Neither does it have any dependence on $n$. We can therefore apply it at the amplitude level. In this case, the sum will be over all pairs of external legs. For instance, we can now deduce that the soft divergence in a general one-loop pure graviton amplitude will be

$$
\frac{r_{\Gamma} \kappa^{2}}{(4 \pi)^{2-\epsilon}} \frac{1}{4 \epsilon^{2}} \sum_{i \neq j}\left(-2 k_{i} \cdot k_{j}\right)^{1-\epsilon} A_{n}^{\text {tree }}
$$

\section{Scalars}

As well as the pure gravity contribution calculated above, we will also need to identify the infrared infinities in amplitudes containing both external gravitons and matter particles. Let us begin with the case with external scalars and gravitons. Fig. 7.D shows all the possible diagrams which could contributed IR divergences. The analysis in all cases closely follows the pure gravity calculation. We find that (a), (b) and (c) all give the same result as the pure gravity case, $(7.13) ;(\mathrm{d})$ and (e) give no soft contribution. 


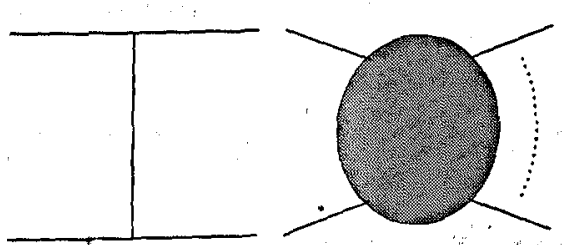

(a)

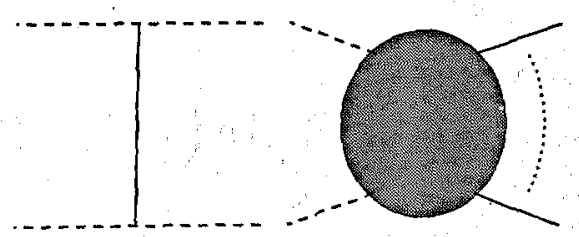

(b)

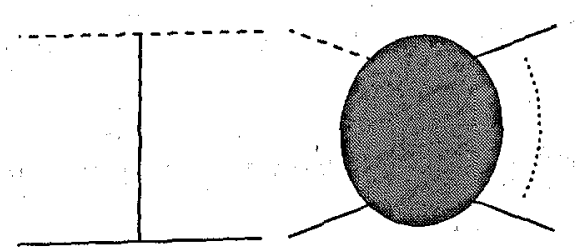

(c)

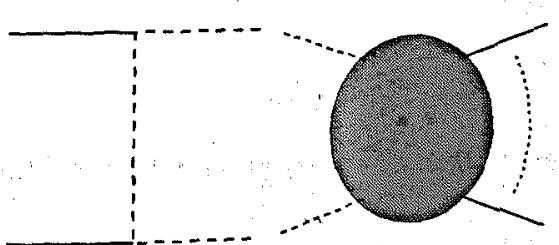

(d)

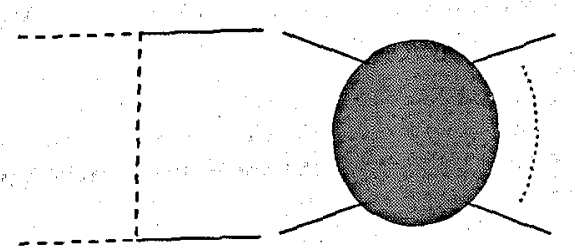

(e)

Figure 7.D: Possible soft contributions to graviton-matter amplitudes. Solid and dashed lines represent gravitons and matter particles,"respectively.

\section{Fermions}

We have to be slightly more careful when looking at amplitudes containing fermions, though again the essential method is the same. In an analogous way to the scalar case, the only relevant diagrams are those in which a graviton joins two fermion legs (fig. 7.D(b)) or a graviton and a fermion leg (fig. 7.D(c)). Consider (b). As before we must add three propagators and two vertices and have an integral of the form

$$
\int \frac{d^{4-2 \epsilon} p C_{a b}\left(k_{1}, k_{2}, p\right)}{\left(p+k_{1}\right)^{2} p^{2}\left(k_{2}-p\right)^{2}} A_{a b}^{(\mathrm{n}-1)-\text { loop }}
$$

Where (writing the spinor indices explicitly)

$$
\left\langle1 | _ { a } \left\langle\left. 2\right|_{b} A_{a b}^{(\mathrm{n}-1)-\mathrm{loop}}\right.\right.
$$

is the the limit of the $n-1$ loop amplitude when the two external legs go on shell. 
$C_{a b}$ takes the form

$$
\begin{aligned}
\kappa^{2}\left\{\langle 1| \frac{i}{8}\left(2 \eta_{\mu \nu}\left(2 \not k_{1}+\not p\right)-\left(2 k_{1}+p\right)_{\mu} \gamma_{\nu}-\left(2 k_{1}+p\right)_{\nu} \gamma_{\mu}\right) i\left(\not k_{1}+\not p\right)\right\}_{a} \\
\times \\
\quad \frac{i}{2}\left(-\delta_{\mu \nu} \delta_{\alpha \beta}+\delta_{\mu \alpha} \delta_{\nu \beta}+\delta_{\mu \beta} \delta_{\nu \alpha}\right) \\
\quad \times\left\{\left(2 \mid \frac{i}{8}\left(2 \eta_{\alpha \beta}\left(2 k_{2}-\not p\right)-\left(2 k_{2}-p\right)_{\alpha} \gamma_{\beta}-\left(2 k_{2}-p\right)_{\beta} \gamma_{\alpha}\right) i\left(k_{2}-\not p\right)\right\}_{b}\right.
\end{aligned}
$$

We can take the $p \rightarrow 0$ limit of this straight away. We also know that"

$$
\langle i| k_{i}=0
$$

via the definitions of the spinors (they must obey the massless Dirac equation), leaving us with

$$
\begin{aligned}
C_{a b}=\frac{i \kappa^{2}}{128}\left\{\langle 1|\left(-2 k_{1 \mu} \gamma_{\nu}-2 k_{1 \nu} \gamma_{\mu}\right) \not k_{1}\right\}_{a} \\
\times\left(\delta_{\mu \alpha} \delta_{\nu \beta}+\delta_{\mu \beta} \delta_{\nu \alpha}\right) \\
\times\left\{\langle 2|\left(-2 k_{2 \beta} \gamma_{\alpha}-2 k_{2 \alpha} \gamma_{\beta}\right) \not_{2}\right\}_{b}
\end{aligned}
$$

Now recall that

$$
\gamma_{\mu} \gamma_{\nu}=-\gamma_{\nu} \gamma_{\mu}+2 g_{\mu \nu}
$$

implying that

$$
\gamma_{\mu} \not k=-k \gamma_{\mu}+2 k_{\mu}
$$

Coupling this with (7.19), we find that (7.20) reduces to

$$
\begin{aligned}
\ddot{C}_{a b} & =i\left\{\left\langle\left. 1\right|_{0}-k_{1 \mu} k_{1 \nu}\right\}_{a}\left(\delta_{\mu \alpha} \delta_{\nu \beta}\right)\left\{\langle 2|-k_{2 \alpha} k_{2 \beta}\right\}_{b}\right. \\
& =i\left(k_{1} \cdot k_{2}\right)^{2}\left\langle1 | _ { a } \left(\left. 2\right|_{b}\right.\right.
\end{aligned}
$$

So, we can see that we get the same result as that for gravitons, equ. (7.13)

In the same way, we can consider case (c). Carrying out a similar calculation as above, we find that, again, we recover expression (7.13). 


\section{Photons}

The method for photon-graviton amplitudes follows the above analysis exactly. The vertices and propagators will differ in places and consideration of helicities is slightly different, but we find that the final results are precisely the same: For diagrams of the form fig. 7.D(b) and (c) we recover (7.13); diagrams (d) and (e) vanish.

Clearly, for all cases involving external matter and external gravitons, we can generalise from single diagrams to full amplitudes. For one-loop results, we will obtain expressions similar to (7.15), possibly with some restriction on the sum. So, we now have the information we require to identify the soft divergences in our calculations.

\subsection{One-Loop Amplitudes with External Gravitons}

Let us use this analysis to look at the four-graviton amplitude. Our calculations in chapter 5 showed that this has a divergent contribution of

$$
A^{[2]}\left(1^{-}, 2^{-}, 3^{+}, 4^{+}\right)=F\left(\frac{2}{\epsilon}\left(\frac{\ln (-u)}{s t}+\frac{\ln (-t)}{s u}+\frac{\ln (-s)}{t u}\right)\right.
$$

when there are gravitons are in the loop, where $F$ is

$$
\frac{i \kappa^{4}(4 \pi)^{\epsilon} r_{\Gamma}}{16(4 \pi)^{2}}\left(\frac{s t\langle 12\rangle^{4}}{\langle 12\rangle\langle 23\rangle\langle 34\rangle\langle 41\rangle}\right)^{2}=\frac{s t u \kappa^{2}(4 \pi)^{\epsilon} r_{\Gamma}}{4(4 \pi)^{2}} A^{\text {tree }}\left(1^{-}, 2^{-}, 3^{+}, 4^{+}\right)
$$

Theoretical considerations implied that this should be pure IR. We can now confirm this using the results from the last section: Summing over all pairs of legs we find that the total IR divergence is.

$$
\left(2(-s)^{1-\epsilon}+2(-t)^{1-\epsilon}+2(-u)^{1-\epsilon}\right) \times \frac{r_{\Gamma}}{(4 \pi)^{2-\epsilon}} \frac{1}{4 \epsilon^{2}} A^{\text {tree }}(--++)
$$

So, the divergent part is

$$
\frac{r_{\Gamma}}{(4 \pi)^{2-\epsilon}}\left(\frac{(s \ln (-s)+t \ln (-t)+u \ln (-u))}{2 \epsilon}\right) A^{\text {tree }}
$$


The infinite part of the amplitude exactly matches our soft result confirming that all observed divergences are IR.

We can also confirm that, as observed, there should be no soft contributions when other particles are in the loop. All these cases would involve trees like fig. 7.D(d), which vanishes.

\subsection{Extracting UV Contributions ${ }^{\dagger}$}

In the next section we will be considering amplitudes which contain both UV and IR divergences. Our interest will be in the UV component, as we discussed in chapter 3 . The path we must take is now clear: We can obtain the IR divergences very easily by the above technique so, having found the total divergence by Cutkosky methods, we can go on to separate the two types and extract the part we are interested in.

† Thanks to Lance Dixon [87] for pointing out this argument. 
Old Bear was in the dining room cutting... Jane Hissey in 'Jolly Snow'

\section{Cutkosky Rules II: Ultraviolet Divergences}

In chapter 6 , we saw that we can obtain complete amplitudes in certain specific cases using cut techniques. In all other cases, the information obtained will give us only a small part of the answer required. This might lead us to conclude that Cutkosky rules are only of use in a few special situations. In fact, we find that even when the conditions of the 'uniqueness theorem' described earlier are not fulfilled we can obtain useful information from cut calculations. In this chapter we will show how we can use cut results to determine exactly the infinities in gravity calculations; in the next we will show how similar reasoning can give us complete amplitudes when the uniqueness conditions are not fulfilled.

Recall that we proved that certain supersymmetric amplitudes could be obtained exactly from the cut results. This result was obtained by looking at a basis covering all possible expressions required to construct particular classes of one-loop amplitudes. In the cutconstructible cases the relevant basis did not allow any ambiguity in the polynomial terms - the terms which could not be obtained directly by using the Cutkosky rules. Let us now look beyond this restricted set to any amplitude, but centre on the infinite parts. Following the same route as before, we can look at the ways that these occur in a general basis. For the four point case, we know that the required basis is the one given in (6.11). Examining this, it is clear that infinite terms only appear in the form

$$
\frac{\left(-s_{i j}\right)^{k-\epsilon}}{\epsilon^{n}}=\frac{\left(-s_{i j}\right)^{k}}{\epsilon^{n}}-\frac{\left(-s_{i j}\right)^{k}}{\epsilon^{n-1}} \ln \left(-s_{i j}\right)+\frac{1}{2} \frac{\left(-s_{i j}\right)^{k}}{\epsilon^{n-2}} \ln \left(-s_{i j}\right)^{2}+\cdots
$$


and, conversely, that $\ln \left(-s_{i j}\right)$ only results from terms

$$
\left(-s_{i j}\right)^{-\epsilon}=1-\epsilon \ln \left(-s_{i j}\right)+\cdots
$$

The implication is that we can easily identify, with no ambiguity, polynomial terms at order $\epsilon^{-n}$ by looking for occurrences of $\ln \left(-s_{i j}\right)$ at order $\epsilon^{-(n-1)}$. (In fact, looking at the complete set of expressions required for one-loop calculations we see that this will be true for any number of external particles.) So, to examine UV and IR infinities in amplitudes, we can simply look at the cut results in the same way as chapter 6 ; we do not require further technology of any kind. .

In this chapter we will use this method to look at divergences in theories of gravity coupled to scalars, fermions and photons. By looking at scattering amplitudes involving external matter particles we will be able to identify some of the problematic infinities in PQG theories. The answer which we will find will enable us to confirm or extend previous derivations of the counter-Lagrangian for each of these theories. We know, from chapter 3 , what form the counterterms should take, up to numerical coefficients. Having identified the infinite components of an amplitude, we will be able to deduce the coefficients necessary to remove the divergences.

\subsection{Gravity Coupled to Scalars}

Let us start with the case considered by 't Hooft and Veltman - gravity coupled to scalars. Initially, we will concentrate on a theory involving only one scalar type. (Note that in this chapter we will implicitly consider real scalars.) We know that divergences will only be seen in amplitudes with external scalars, so let us look at the scattering of four onshell scalars. The calculation can be split into two parts - the cut contributions from (a) gravitons and (b) scalars 'in the loop' (see fig. 8.A).

The easier of these two is the latter; it requires us to sew together two trees with four external scalars. Since there is a symmetry amongst the external particles, we expect to 


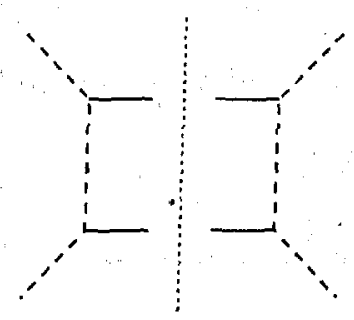

(a)

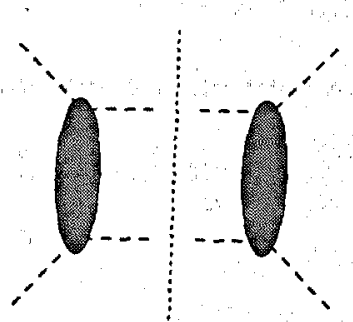

(b)

Figure 8.A: Cut contributions required for the four scalar calculation.

have to carry out essentially the same calculation in all channels. Hence, for simplicity, we will look at the $s$-channel case and deduce the total result from this.

The tree amplitude needed for this cut can be found by direct calculation. It is

$$
A\left(\phi_{1}, \phi_{2}, \phi_{3}, \phi_{4}\right)=-\frac{i \kappa^{2}}{2}\left(\frac{1}{s}\left(t^{2}+u^{2}\right)+\frac{1}{t}\left(s^{2}+u^{2}\right)+\frac{1}{u}\left(s^{2}+t^{2}\right)\right) .
$$

Clearly, this leads to the cut expression

$$
\begin{aligned}
A\left(1,2, l_{1}, l_{2}\right) A\left(l_{2}, l_{1}, 3,4\right) & \\
= & -\frac{\kappa^{4}}{4}\left(\frac{1}{s}\left(4\left(2 . l_{1}\right)^{2}+4\left(1 . l_{1}\right)^{2}\right)+\frac{1}{2\left(2 . l_{1}\right)}\left(s^{2}+4\left(1 . l_{1}\right)^{2}\right)+\frac{1}{2\left(1 . l_{1}\right)}\left(4\left(2 . l_{1}\right)^{2}+s^{2}\right)\right) \\
& \times\left(\frac{1}{s}\left(4\left(3 . l_{1}\right)^{2}+4\left(4 . l_{1}\right)^{2}\right)-\frac{1}{2\left(3 . l_{1}\right)}\left(s^{2}+4\left(4 . l_{1}\right)^{2}\right)-\frac{1}{2\left(4 . l_{1}\right)}\left(4\left(3 . l_{1}\right)^{2}+s^{2}\right)\right) .
\end{aligned}
$$

It is straightforward to expand this. We find a sum of scalar boxes, quadratic triangles, and quartic bubbles. The process of integration follows the graviton scattering calculation: We convert the $\int d \mathrm{LIPS}$ to $\int d^{4} l_{1}$ by introducing extra propagators and evaluate this directly.

We find that the $s$-channel cuts with scalars in the loop are

$$
\begin{aligned}
\frac{i \kappa^{4} r_{\Gamma}}{(4 \pi)^{2-\epsilon}}\left(\frac{1}{2 u t}\left(3 u^{2} t^{2}+2 u^{3} t+2 u t^{3}+u^{4}+t^{4}\right) \ln (s) \frac{1}{\epsilon}\right. \\
+\frac{1}{4}\left(3 u t+2 u^{2}+2 t^{2}\right) \ln (s)^{2}+\frac{s^{3} \ln (s) \ln (t)}{2 t} \\
\left.+\frac{1}{240}\left(-161 t^{2}+39 u t-161 u^{2}\right) \ln (s)+\frac{s^{3} \ln (s) \ln (u)}{2 u}\right) .
\end{aligned}
$$

To obtain the graviton contribution, we must consider sewing trees with two external scalars and two external gravitons. As before, we will have a contribution in every channel. 
But, again, symmetry implies that we can concentrate on the $s$-channel and deduce the others from this. The subtlety in this case is that we must take into account the different possible choices of graviton helicities across the cut. In fact, if the helicities of the gravitons are the same then the tree amplitudes vanish

$$
A\left(\phi_{1}, 2^{+}, 3^{+}, \phi_{4}\right)=A\left(\phi_{1}, 2^{-}, 3^{-}, \phi_{4}\right)=0
$$

So, we need only look at the two cases in which the internal legs have differing helicities (cf, the graviton scattering case; fig. 6.G). The two helicity choices are

$$
A\left(\phi_{1}, \phi_{2}, l_{2}^{-}, l_{1}^{+}\right) A\left(\phi_{3}, \phi_{4}, l_{1}^{-}, l_{2}^{+}\right)
$$

and

$$
A\left(\phi_{1}, \phi_{2}, l_{2}^{+}, l_{1}^{-}\right) A\left(\phi_{3}, \phi_{4}, l_{1}^{+}, l_{2}^{-}\right)
$$

We can show that these are equivalent if we make the substitutions $l_{2} \rightarrow-l_{1}$ and $l_{1} \rightarrow-l_{2}$. These substitutions leave the integration unchanged, so the result from both choices will be the same. We will calculate the cuts for one choice and double the result to get the required contribution.

We have derived the tree amplitude needed for this calculation already; it was used when we looked at cuts in graviton scattering amplitudes. Recall that it is

$$
A\left(\phi_{1}, 2^{-}, 3^{+}, \phi_{4}\right)=\frac{i \kappa^{2}}{4} \frac{\langle 12\rangle^{4}\langle 42\rangle^{4}}{(\langle 12\rangle\langle 23\rangle\langle 34\rangle\langle 41\rangle)^{2}} \frac{s t}{u} \text {. }
$$

The resulting cut expression is

$$
\begin{aligned}
A\left(\phi_{1}, \phi_{2}, l_{1}^{-}, l_{2}^{+}\right) A\left(\phi_{3}, \phi_{4}, l_{2}^{-}, l_{1}^{+}\right) & s\left(1 . l_{1}\right) \\
& =-\frac{\kappa^{4}}{16} \frac{\left\langle 1 l_{1}\right\rangle^{4}\left\langle 2 l_{1}\right)^{4}}{\left(\left\langle 1 l_{1}\right\rangle\left\langle l_{1} l_{2}\right\rangle\left\langle l_{2} 2\right\rangle\langle 21\rangle\right)^{2}} \frac{\left(4 l_{1}\right\rangle^{4}\left\langle 3 l_{1}\right\rangle^{4}}{\left(1 . l_{2}\right)} \times \frac{s\left(3 . l_{2}\right)}{\left(\left\langle 3 l_{2}\right\rangle\left\langle l_{2} l_{1}\right\rangle\left\langle l_{1} 4\right\rangle\langle 43\rangle\right)^{2}} \frac{\left(3 . l_{1}\right)}{} \\
& =-\frac{\kappa^{4}}{256 s^{2}} \frac{\left(\operatorname{tr}_{+}\left(2 l_{2} 4 l_{1}\right)\right)^{4}}{\left(l_{1} .1\right)\left(l_{1} .2\right)\left(l_{1} .3\right)\left(l_{1} .4\right)}
\end{aligned}
$$

To evaluate this recall that

$$
\operatorname{tr}_{+}\left(2 l_{2} 4 l_{1}\right)=\frac{1}{2} \operatorname{tr}\left(2 l_{2} 4 l_{1}\right)+\frac{1}{2} \epsilon\left(2 l_{2} 4 l_{1}\right) .
$$


Using the on-shell condition of the trees, $l_{2}=l_{1}+k_{1}+k_{2}$, we can write $\epsilon\left(2 l_{2} 4 l_{1}\right)$ as

$$
\begin{aligned}
\epsilon\left(2 l_{2} 4 l_{1}\right) & =\epsilon\left(2 l_{1} 4 l_{1}\right)+\epsilon\left(214 l_{1}\right) \\
& =\epsilon\left(214 l_{1}\right)
\end{aligned}
$$

and

$$
\frac{1}{2} \operatorname{tr}\left(2 l_{2} 4 l_{1}\right)=\frac{1}{2} \operatorname{tr}\left(214 l_{1}\right)+4\left(4 . l_{1}\right)\left(2 . l_{1}\right)
$$

Hence,

$$
\operatorname{tr}_{+}\left(2 l_{2} 4 l_{1}\right)=4\left(4 . l_{1}\right)\left(2 . l_{1}\right)+\frac{1}{2} \operatorname{tr}\left(214 l_{1}\right)+\frac{1}{2} \epsilon\left(214 l_{1}\right)
$$

In addition, we know that

$$
\int d \operatorname{LIPS} \epsilon\left(214 l_{1}\right) f\left(1,2,3,4, l_{1}\right)=0
$$

if $f$ contains only dot-products of the momenta (ie it has no $\epsilon$-tencrs) and that a product of $2 \epsilon$ tensors looks like a sum of dot-products (see appendix VI). This implies that we can ignore products of an odd number of $\epsilon$ 's, allowing us to expand $\left(\operatorname{tr}_{+}\left(2 l_{2} 4 l_{1}\right)\right)^{4}$ as

$$
\begin{aligned}
& \left(\left(\frac{1}{2} \operatorname{tr}\left(214 l_{1}\right)\right)^{2}+\left(\frac{1}{2} \epsilon\left(214 l_{1}\right)\right)^{2}+\left(4\left(4 . l_{1}\right)\left(2 . l_{1}\right)\right)^{2}+4\left(4 . l_{1}\right)\left(2 . l_{1}\right) \operatorname{tr}\left(214 l_{1}\right)\right)^{2} \\
& \quad+4\left(\frac{1}{2} \epsilon\left(214 l_{1}\right)\right)^{2}\left(\left(4\left(4 . l_{1}\right)\left(2 . l_{1}\right)\right)^{2}+4\left(4 . l_{1}\right)\left(2 . l_{1}\right) \operatorname{tr}\left(214 l_{1}\right)+\left(\frac{1}{2} \operatorname{tr}\left(214 l_{1}\right)\right)^{2}\right) .
\end{aligned}
$$

In appendix VI we show how $\left(\frac{1}{2} \epsilon\left(214 l_{1}\right)\right)^{2}$ can be expanded as

$$
\begin{aligned}
\left(\frac{1}{2} \epsilon\left(214 l_{1}\right)\right)^{2}=4\left(\left(l_{1} .2\right)^{2} t^{2}\right. & +\left(l_{1} .1\right)^{2} u^{2}+\left(l_{1} .4\right)^{2} s^{2} \\
& \left.-2\left(l_{1} .2\right) t\left(l_{1} .1\right) u-2\left(l_{1} .2\right) t\left(l_{1} .4\right) s-2\left(l_{1} .1\right) u\left(l_{1} .4\right) s\right)
\end{aligned}
$$

Inserting this, together with the expansion for $\operatorname{tr}\left(214 l_{1}\right)$

$$
\operatorname{tr}\left(214 l_{1}\right)=4\left(\left(k_{2} \cdot k_{1}\right)\left(k_{4} \cdot l_{1}\right)-\left(k_{2} \cdot k_{4}\right)\left(k_{1} \cdot l_{1}\right)+\left(k_{4} \cdot k_{1}\right)\left(k_{2} \cdot l_{1}\right)\right)
$$

in (8.16), we can see that (8.10) can be written as a sum of scalar boxes, quadratic triangles and quartic bubbles. 
Doubling this, as discussed, and carrying out the integrations, we find the cuts for internal gravitons to be

$$
\begin{gathered}
\frac{i \kappa^{4} r_{\Gamma}}{(4 \pi)^{2-\epsilon}}\left(\frac{1}{4}\left(u^{2}-u t+t^{2}\right) \ln (s)^{2}-\frac{1}{120}\left(u^{2}+41 u t+t^{2}\right) \ln (s)\right. \\
\left.+\frac{t^{3} \ln (t) \ln (s)}{2 s}+\frac{u^{3} \ln (u) \ln (s)}{2 s}\right)
\end{gathered}
$$

Adding the internal scalar (8.5) and internal graviton (8.19) contributions, we find the total $s$-channel cuts to be

$$
\begin{gathered}
\frac{i \kappa^{4} r_{\Gamma}}{(4 \pi)^{2-\epsilon}}\left(\frac{\left(u^{4}+2 u^{3} t+2 u t^{3}+3 u^{2} t^{2}+t^{4}\right) \ln (s)}{2 u t} \frac{1}{\epsilon}+\frac{1}{4}\left(3 u^{2}+2 u t+3 t^{2}\right) \ln (s)^{2}\right. \\
+\frac{\left(2 t^{4}+u^{4}+4 u t^{3}+4 u^{3} t+6 u^{2} t^{2}\right) \ln (s) \ln (t)}{2 t s} \\
+\frac{\left(4 u t^{3}+4 u^{3} t+6 u^{2} t^{2}+t^{4}+2 u^{4}\right) \ln (s) \ln (u)}{2 u s} \\
\left.+\frac{1}{240} \frac{\left(-163 u^{3} t-43 u^{2} t^{2}-163 u t^{3}\right) \ln (s)}{u t}\right)
\end{gathered}
$$

As we indicated, the total contribution comes from the combined contribution in all channels. (The $t$ and $u$ cases are found simply by substituting $s \leftrightarrow t$ and $s \leftrightarrow u$, respectively.) As expected, when we reconstruct the full result, we can identify a soft term of the form

$$
\begin{aligned}
& \frac{r_{\Gamma}}{2(4 \pi)^{2-\epsilon}} \kappa^{2}\left((-s)^{1-\epsilon}+(-t)^{1-\epsilon}+(-u)^{1-\epsilon}\right)\left(\frac{1}{\epsilon^{2}}+\mathcal{O}(\epsilon)\right) A^{\text {tree }}(--++) \\
& \quad=\frac{i r_{\Gamma} \kappa^{4}}{2 \epsilon(4 \pi)^{2-\epsilon}}(s \ln (s)+t \ln (t)+u \ln (u)) \frac{\left(u^{4}+2 u^{3} t+2 u t^{3}+3 u^{2} t^{2}+t^{4}\right)}{s t u} .
\end{aligned}
$$

related to the $1 / \epsilon$ term in (8.20). If we remove this IR contribution, then we can deduce that the UV infinite component in this amplitude is

$$
\frac{i \kappa^{4} r_{\Gamma}}{(4 \pi)^{2}} \frac{203}{160} \frac{1}{\epsilon}\left(s^{2}+t^{2}+u^{2}\right)
$$

So, by looking at the straightforward cut calculation we have found a complete, and important, part of the amplitude.

We can use this as a test of 't Hooft and Veltman's counter-Lagrangian [56]: It should be removed exactly by a tree contribution from the new terms which they derived. To see 


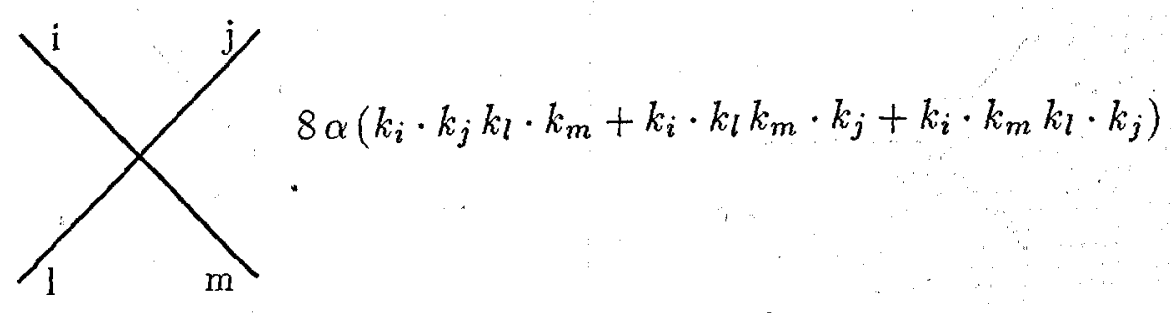

Figure 8.B: The four-point scalar vertex.

that this is the case, note that, to first order, $\left(\partial_{\mu} \phi \partial^{\mu} \phi\right)^{2}$ produces a four point Feynman 'vertex (fig. 8.B). So, the counterterm

$$
\Delta \mathcal{L}=-\frac{\sqrt{-g}}{(4 \pi)^{2}} \frac{203}{30} \frac{1}{\epsilon} R^{2}=-\frac{\kappa^{4} \sqrt{-g}}{(4 \pi)^{2}} \frac{203}{320} \frac{1}{\epsilon}\left(\partial_{\mu} \phi \partial^{\mu} \phi\right)^{2}
$$

will produce a term

$$
-\frac{i \kappa^{4}}{(4 \pi)^{2}} \frac{203}{160} \frac{1}{\epsilon}\left(s^{2}+t^{2}+u^{2}\right)
$$

This does cancel (8.22), as required. (Note that $r_{\Gamma}=1+\mathcal{O}(\epsilon)$ and effects of dimensional regularisation on $\kappa$ do not effect the divergent terms.) Thus we have confirmed the result in ref. [56] for the on-shell case.

We can also look at the result of adding extra scalars to our theory. The cut technique allows us to see this effect with relative ease; the above calculation will differ only by the addition of an extra cut contribution coming from an internal loop of the new scalars. To obtain fuller information we must also look at the interaction between two pairs of different scalars. We will see that we can use parts of the above calculation to reduce the work required here.

't Hooft and Veltman did not give an explicit result for the counter-Lagrangian in the theory with multiple scalars, so let us begin by considering what their method implies that we should see in this 'case. Starting with 't Hooft and Veltman's counter-Lagrangian, let us consider the effect of changing the scalar, $\phi$ to a vector of scalars, $\phi_{i}$. There are three differences which we must take into account. 


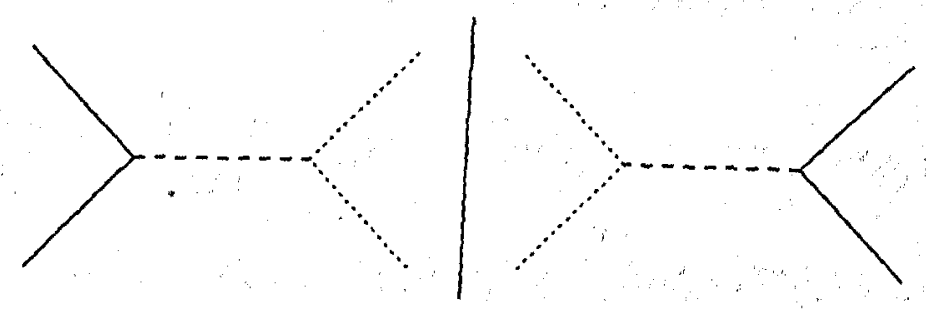

Figure 8.C: One channel cut for four scalar amplitude with internal loop of another scalar type.

First, we know that from their argument that we must also add a factor of

$$
-\frac{\sqrt{-g}}{(4 \pi)^{2}} \frac{1}{\epsilon}\left(\frac{1}{144} R^{2}+\frac{1}{120} R_{\mu \nu} R^{\mu \nu}-\frac{1}{360} R^{2}\right)
$$

to the counter-Lagrangian for each extra degree of freedom in the vector. We can confirm this step by looking at the effect of adding an internal loop of a new scalar to diagrams with four external scalars. For this we have a cut of the form shown in fig. 8.C. Calculation in one channel produces the infinite contribution

$$
\frac{i \kappa^{4}}{(4 \pi)^{2}} \frac{1}{480} \frac{1}{\epsilon}\left(t^{2}+u^{2}+s^{2}\right)
$$

and, summing over all channels, we find the total divergence

$$
\frac{i \kappa^{4}}{(4 \pi)^{2}} \frac{1}{160} \frac{1}{\epsilon}\left(t^{2}+u^{2}+s^{2}\right)
$$

This corresponds correctly to the results expected from the new term (8.25).

Second, we should note that the equations of motion will become

$$
\begin{aligned}
D_{\mu} D^{\mu} \phi_{i} & =0 \\
R_{\mu \nu} & =\frac{\kappa^{2}}{2}\left(D_{\mu} \phi_{i}\right)\left(D_{\nu} \phi_{i}\right) \\
\quad R & =\frac{\kappa^{2}}{2}\left(D_{\mu} \phi_{i}\right)\left(D^{\mu} \phi_{i}\right)
\end{aligned}
$$

Third, we must be careful about the occurrence of terms like $\left(D_{\mu} \phi D_{\nu} \phi\right)\left(D^{\mu} \phi D^{\nu} \phi\right)$ and $\left(D_{\mu} \phi D^{\mu} \phi\right)\left(D_{\nu} \phi D^{\nu} \phi\right)$. These are equivalent when $\phi$ is a single scalar, but differ when it is a vector. So, there will be cancellations in the derivation of the counter-Lagrangian for one scalar which aren't seen when we add more flavours to the theory. 
The combination of these observations leads us to expect the counter-Lagrangian to take the form

$$
\begin{array}{r}
-\frac{\sqrt{-g}}{(4 \pi)^{2}} \frac{1}{\epsilon}\left(\frac{2+n}{240} R^{2}+\frac{42+n}{.120} R_{\mu \nu} R^{\mu \nu}+\alpha \kappa^{4}\left(\partial_{\mu} \phi_{i} \partial^{\mu} \phi_{i}\right)\left(\partial_{\nu} \phi_{j} \partial^{\nu} \phi_{j}\right)\right. \\
\left.+\beta \kappa^{4}\left(\partial_{\mu} \phi_{i} \partial_{\nu} \phi_{i}\right)\left(\partial^{\mu} \phi_{j} \partial^{\nu} \phi_{j}\right)+\frac{\kappa^{2}}{12} R\left(\partial_{\mu} \phi_{i} \partial^{\mu} \phi_{i}\right)+2 \kappa^{2}\left(D_{\mu} D^{\mu} \phi_{i}\right)^{2}\right)
\end{array}
$$

for a general $\phi_{i}$. Note that the cancellations seen in the single scalar case have left us ,with an ambiguity - $\alpha$ and $\beta$ cannot be deduced directly from the previous result. We will determine them here using cut results. We can find a useful condition by comparing (8.29) with the single scalar expression: Setting $\phi_{i}=\phi$ and $n=1$ leads us to deduce that $\alpha+\beta=\frac{1}{2}$.

We will be calculating on-shell amplitudes, so we should look at the explicit on-shell form of the above counter-Lagrangian expression. If we insert the field equations, then we find this simplifies to become

$$
\begin{aligned}
\Delta \mathcal{L}=-\frac{\kappa^{4} \sqrt{-g}}{(4 \pi)^{2}-\epsilon}\left(\left(\frac{42+n}{960}+\alpha\right)\left(\partial_{\mu} \phi_{i} \partial^{\mu} \phi_{i}\right)\left(\partial_{\nu} \phi_{j} \partial^{\nu} \phi_{j}\right)\right. \\
\left.+\left(\frac{42+n}{480}+\beta\right)\left(\partial_{\mu} \phi_{i} \partial_{\nu} \phi_{i}\right)\left(\partial^{\mu} \phi_{j} \partial^{\nu} \phi_{j}\right)\right) \\
\quad-
\end{aligned}
$$

So, our goal of finding the counter-Lagrangian for multiple scalars now reduces to finding the values of $\alpha$ and $\beta$. To determine these, let us turn to an amplitude with two different pairs of external scalars. If $k_{1}$ and $k_{2}$ are the momenta of one pair and $k_{3}$ and $k_{4}$ the momenta of the others, then we must consider (a) the $s$-channel cut with gravitons in the loop (b) the two $s$-channel cuts with identical scalars in the loop (one diagram for each sort of scalar) and (c) the $t$ - and $u$-channel cuts with one scalar of each type in the loop (see fig. 8.D).

(a) will be precisely the same as the result in the case with a single scalar (8.20). The other two are straightforward and the calculations follow similar steps to those above: (b) evaluates to give

$$
\frac{i \kappa^{4} r_{\Gamma}}{(4 \pi)^{2-\epsilon}}\left(\frac{t u}{2 \epsilon} \ln (s)-t u \ln (s)^{2}-\frac{1}{120}\left(21 t^{2}-139 u t+21 u^{2}\right) \ln (s)\right)
$$




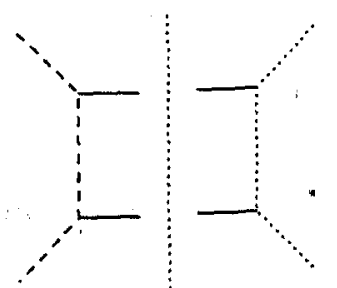

(a)

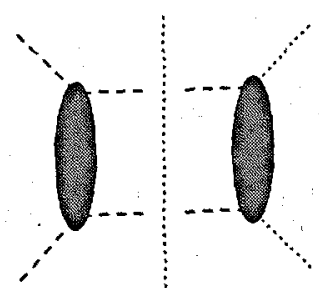

(b)

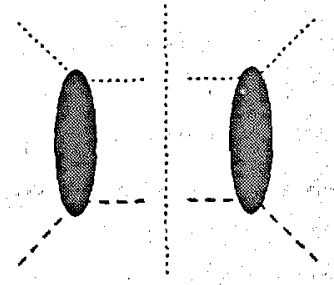

(c)

Figure 8.D: Cut contributions for an amplitude with two pairs of different external scalars.

and the $u$-channel part of (c) is

$$
\frac{i \kappa^{4} r \Gamma}{(4 \pi)^{2-\epsilon}}\left(\frac{t u^{2}}{2 s} \frac{1}{\epsilon} \ln (u)-\frac{u^{2}}{4} \ln (u)-\frac{u^{3}}{2 s} \ln (u) \ln (s)+\frac{u^{2}}{4} \ln (u)^{2}\right) .
$$

Symmetry implies that the $t$-channel contribution can be deduced directly from this by making the substitution $t \leftrightarrow u$.

Summing over all channels, we can find the total divergent contribution. We can extract the IR infinities as before. They resemble those in the single scalar case, but restricted to the s-channel. This leaves us with a UV divergence of

$$
-\frac{i \kappa^{4}}{(4 \pi)^{2}} \frac{1}{\epsilon}\left(-\frac{101}{120} u^{2}+\frac{49}{120} s^{2}-\frac{101}{120} t^{2}\right)
$$

(Note that since

$$
\begin{aligned}
\sum_{i, j}\left(\sigma\left(\partial_{\mu} \phi_{i} \partial^{\mu} \phi_{i}\right)\left(\partial_{\nu} \phi_{j} \partial^{\nu} \phi_{j}\right)+\rho\left(\partial_{\mu} \phi_{i} \partial_{\nu} \phi_{i}\right)\left(\partial^{\mu} \phi_{j} \partial^{\nu} \phi_{j}\right)\right) & \\
= & \sum_{i}(\sigma+\rho)\left(\partial_{\mu} \phi_{i} \partial^{\mu} \phi_{i}\right)^{2} \\
& +\sum_{i \neq j}\left(\sigma\left(\partial_{\mu} \phi_{i} \partial^{\mu} \phi_{i}\right)\left(\partial_{\nu} \phi_{j} \partial^{\nu} \phi_{j}\right)+\rho\left(\partial_{\mu} \phi_{i} \partial_{\nu} \phi_{i}\right)\left(\partial^{\mu} \phi_{j} \partial^{\nu} \phi_{j}\right)\right)
\end{aligned}
$$

we have an easy check that this is consistent with the single scalar results - the sum of the coefficients must be the same as the result for four external scalars of the same type (in a theory with 2 scalars). This we can confirm to be the case.)

We are now in a position to find $\alpha$ and $\beta$ by equating coefficients of $s$ and $t$ in the counter-Lagrangian with those required to cancel (8.33). The resulting equations are

$$
2\left(\frac{2+42}{960}+\alpha\right)=-\frac{49}{120} \quad\left(\frac{42+2}{480}+\beta\right)=\frac{101}{120}
$$


which imply that

$$
\alpha=-\frac{1}{4} \quad \beta=\frac{3}{4}
$$

(As expected, $\alpha+\beta=\frac{1}{2}$ ) So, we can deduce that the counter-Lagrangian for $n$ scalars takes the form

$$
\begin{aligned}
-\frac{\sqrt{-g}}{\epsilon}\left(\frac{2+n}{240} R^{2}\right. & +\frac{42+n}{120} R_{\mu \nu} R^{\mu \nu}-\frac{\kappa^{4}}{4}\left(\partial_{\mu} \phi_{i} \partial^{\mu} \phi_{i}\right)\left(\partial_{\nu} \phi_{j} \partial^{\nu} \phi_{j}\right) \\
& \left.+\frac{3}{4} \kappa^{4}\left(\partial_{\mu} \phi_{i} \partial_{\nu} \phi_{i}\right)\left(\partial^{\mu} \phi_{j} \partial^{\nu} \phi_{j}\right)+\frac{\kappa^{2}}{12} R\left(\partial_{\mu} \phi_{i} \partial^{\mu} \phi_{i}\right)+2 \kappa^{2}\left(D_{\mu} D^{\mu} \phi_{i}\right)^{2}\right)
\end{aligned}
$$

\subsection{Gravity Coupled to Fermions}

The process for obtaining fermion cuts follows the scalar calculation very closely. Again, we look at the case with four external matter particles and, again, we have contributions from the gravitons 'in the loop' and fermions 'in the loop' cases. (Diagrammatically, these are the same as the scalar case, fig. 8.A.) The subtlety is that the matter particles in this case also have helicity which must be taken into account. As with graviton scattering amplitudes, we can consider the different helicity configurations of the external particles. It is easy to show that all cuts vanish for external helicities $(-,+,+,+)$ and $(+,+,+,+)$, implying that the amplitudes will be divergence free. We will concentrate on the case $\left(1^{-}, 2^{+}, 3^{-}, 4^{+}\right)$(with chiral fermions).

The asymmetry of the external helicities in $A\left(1^{-}, 2^{+}, 3^{-}, 4^{+}\right)$implies that, unlike in the scalar amplitude, different channels will have different contributions. In fact, there are two cases which must be considered: the $u$-and $s$-channels. (The $s$ and $t$ calculations will be related by symmetry:)

In the $u$-channel, the internal graviton contribution is zero; there is no internal helicity configuration for which all the trees involved in the calculation are non-zero. So, the only contribution which we must calculate is the one with internal fermions. The building block for that case will be the tree with four external fermions. The only choice of external 
helicities for which this is non-zero is $A^{\text {tree }}(-,+,-,+)$. We can find this from direct calculation:

$$
A\left(1^{-}, 2^{+}, 3^{-}, 4^{+}\right)=-\frac{i(13)[24] \kappa^{2}}{8 s t}\left(2 s^{2}+s t+2 t^{2}\right)
$$

Sewing two such trees together gives us the cut contribution

$$
\begin{aligned}
& A\left(1-l_{1}^{+} 3^{-} l_{2}^{+}\right) A\left(l_{1}^{-} 2^{+} l_{2}^{-} 4^{+}\right)= \\
& -\frac{\kappa^{4}}{16} \frac{\langle 13\rangle^{2}\left[l_{1} l_{2}\right]}{4\left(l_{1} .1\right)\left(l_{1} .3\right)}\left(u^{2}+12\left(l_{1} .1\right)^{2}+12\left(l_{1} .3\right)^{2}\right) \frac{[24]^{2}\left\langle l_{1} l_{2}\right\rangle}{4\left(l_{1} .2\right)\left(l_{1} .4\right)}\left(u^{2}+12\left(l_{1} .2\right)^{2}+12\left(l_{1} .4\right)^{2}\right) \\
& \quad=-\frac{\kappa^{4}}{16}[24]\langle 13\rangle u \frac{\left(u^{2}+12\left(l_{1} .1\right)^{2}+12\left(l_{1} .3\right)^{2}\right)\left(u^{2}+12\left(l_{1} .2\right)^{2}+12\left(l_{1} .4\right)^{2}\right)}{16\left(l_{1} .1\right)\left(l_{1} .2\right)\left(l_{1} .3\right)\left(l_{1} .4\right)} \\
& \quad=-\frac{\kappa^{4} u^{3}}{16\langle 24\rangle[13]} \frac{\left(u^{2}+.12\left(l_{1} .1\right)^{2}+12\left(l_{1} .3\right)^{2}\right)\left(u^{2}+12\left(l_{1} .2\right)^{2}+12\left(l_{1} .4\right)^{2}\right)}{16\left(l_{1} .1\right)\left(l_{1} .2\right)\left(l_{1} .3\right)\left(l_{1} .4\right)}
\end{aligned}
$$

So, expanding this and integrating in the usual way gives us the total $u$-channel cuts to be:

$$
\begin{array}{r}
-\frac{i r_{\Gamma} \kappa^{4}}{(4 \pi)^{2-\epsilon}} \frac{1}{16\langle 24\rangle[13]}\left(\frac{u^{3}\left(2 t^{2}+s t+2 s^{2}\right) \ln (u)}{s t}+\frac{2 u^{4} \ln (u) \ln (t)}{t}\right. \\
\left.+\frac{2 u^{4} \ln (u) \ln (s)}{s}+\frac{3}{2} u^{3} \ln (u)^{2}-\frac{9}{8} u^{3} \ln (u)\right)
\end{array}
$$

The $s$-channel has contributions for both particle types in the loop. Let us begin with the fermion contribution. Again, we use (8.38) as our building block. The cut expression in this case is

$$
\begin{aligned}
& A\left(1-2^{+} l_{1}^{-} l_{2}^{+}\right) A\left(l_{2}^{-} l_{1}^{+} 3^{-} 4^{+}\right)= \\
& -\frac{\kappa^{4}}{64} \frac{\left\langle 1 l_{1}\right\rangle\left[2 l_{2}\right]\left(3 l_{2}\right\rangle\left[l_{1} 4\right]}{s^{2}\left(3 . l_{1}\right)\left(2 . l_{1}\right)}\left(3 s^{2}+12\left(2 . l_{1}\right)^{2}+4\left(1 . l_{1}\right)^{2}\right)\left(3 s^{2}+4\left(4 . l_{1}\right)^{2}+12\left(3 . l_{1}\right)^{2}\right) \\
& \quad=-\frac{\kappa^{4}}{64} \frac{\operatorname{tr}-\left(1 l_{1} 42 l_{2} 3\right)}{\langle 24\rangle[13]} \frac{\left(3 s^{2}+12\left(2 . l_{1}\right)^{2}+4\left(1 . l_{1}\right)^{2}\right)\left(3 s^{2}+4\left(4 . l_{1}\right)^{2}+12\left(3 . l_{1}\right)^{2}\right)}{s^{2}\left(2 . l_{1}\right)\left(3 . l_{1}\right)}
\end{aligned}
$$

We can expand the trace as

$$
\begin{aligned}
\operatorname{tr}-\left(1 l_{1} 42 l_{2} 3\right) & =\operatorname{tr}-\left(1 l_{1} 42 l_{1} 3\right)-\operatorname{tr}-\left(1 l_{1} 4243\right) \\
& =2\left(l_{1} .4\right) \operatorname{tr}\left(12 l_{2} 3\right)-2\left(2 . l_{1}\right) \operatorname{tr}\left(14 l_{1} 3\right)-2(2.4) \operatorname{tr}\left(1 l_{1} 43\right)
\end{aligned}
$$


Using this we can evaluate the cut as before and find

$$
\begin{gathered}
-\frac{i \kappa^{4} r_{\Gamma}}{(4 \pi)^{2-\epsilon}} \frac{1}{16\langle 24\rangle[13]}\left(\frac{1}{\epsilon} \frac{u^{2}\left(2 u^{2}+3 u t+3 t^{2}\right)}{t} \ln (s)-\frac{\left(t^{3}+2 u t^{2}+2 u^{2} t-u^{3}\right)}{2} \ln (s)^{2}\right. \\
\left."-\frac{s^{2}\left(2 u^{2}-t^{2}\right)}{t} \ln (s) \ln (t)+\frac{u\left(274 u t-53 u^{2}+60 t^{2}\right)}{60} \ln (s)\right) .
\end{gathered}
$$

The tree used for the cut with internal gravitons was used in chapter 6 . We can write it as

$$
A\left(g^{-}, f^{-}, f^{+}, g^{+}\right)=\frac{\langle 13\rangle^{3}}{\langle 12\rangle^{3}} A\left(g^{-}, g^{-}, g^{+}, g^{+}\right)=\frac{i \kappa^{2}}{4} \frac{\langle 12\rangle^{3}\langle 13\rangle^{3}}{\langle 23\rangle^{2}\langle 34\rangle^{2}\langle 14\rangle^{2}} \frac{s t}{u}
$$

This is very similar to the tree in the four scalar calculation with internal gravitons. In fact, the calculation process here follows that case very closely, so we will not give details. The result is

$$
\begin{aligned}
-\frac{i \kappa^{4} r_{\Gamma}}{(4 \pi)^{2-\epsilon}} & \frac{1}{\langle 24\rangle[13]}\left(\frac{u^{4} \ln (s) \ln (u)}{8 s}-\frac{1}{32}\left(4 t^{3}+2 s^{3}+11 t^{2} s+8 t s^{2}\right) \ln (s)^{2}\right. \\
& \left.-\frac{t^{2}\left(2 t s+2 t^{2}+s^{2}\right) \ln (s) \ln (t)}{16 s}-\frac{1}{960} u\left(7 s^{2}-5 t s+48 t^{2}\right) \ln (s)\right) .
\end{aligned}
$$

Summing (8.43) and (8.45), we see that the total s-channel cuts are

$$
\begin{array}{r}
-\frac{i \kappa^{4} r_{\Gamma}}{(4 \pi)^{2-\epsilon}} \frac{u}{16\langle 24)[13]}\left(\frac{1}{\epsilon} \frac{u^{2}\left(2 u^{2}+3 u t+3 t^{2}\right)}{t} \ln (s)+\frac{1}{4} u(21 s+4 t) \ln (s)\right. \\
+\frac{2 u^{3} \ln (s) \ln (u)}{s}+\frac{\left(2 s^{4}+2 t s^{3}-t^{2} s^{2}+2 s t^{3}+2 t^{4}\right) \ln (s) \ln (t)}{s t} \\
\left.+\frac{1}{2}\left(3 s^{2}+10 s t+6 t^{2}\right) \ln (s)^{2}\right) .
\end{array}
$$

We can find the $t$-channel contribution from this by making the exchange $s \leftrightarrow t$. If we combine the results from the three channels we can deduce the total expression. As expected, we have an IR contribution,

$$
\begin{aligned}
& -\frac{i \kappa^{4} r_{\Gamma}}{2(4 \pi)^{2-\epsilon}} \frac{1}{\epsilon}(u \ln (u)+s \ln (s)+t \ln (t)) \frac{1}{8} \frac{u^{2}\left(s t+2 t^{2}+2 s^{2}\right)}{\langle 24)[13] s t} .
\end{aligned}
$$

Removing this leaves the UV component easily identifiable. We find this to be

$$
-\frac{59}{128} \frac{i \kappa^{4} r_{\Gamma}}{(4 \pi)^{2}} \frac{1}{\epsilon} \frac{u^{3}}{\langle 24\rangle[13]}
$$




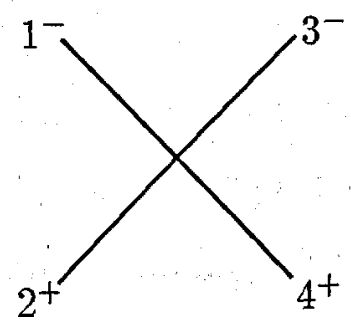

Figure 8.E: Four-point counterterm vertex for fermions.

This expression must be cancelled by a four-point counterterm. This case has not been considered before, so we can use our result to deduce new information about the DiracEinstein counter-Lagrangian. The general on-shell counterterm which we must consider takes the form

$$
\frac{\alpha}{2} \frac{\kappa^{4}}{(4 \pi)^{2}} \frac{e}{\epsilon}\left(\bar{\eta} \gamma_{\mu} \overleftrightarrow{\partial}_{\nu} \eta\right)^{2}
$$

(Note that other all other possibilities can be related to this by a combination of on-shell conditions, integration by parts and the Fierz theorem.) At lowest order, this leads us to the vertex in fig. 8.E. We can deduce a counterterm contribution

$$
\begin{aligned}
\frac{i \kappa^{4}}{(4 \pi)^{2}} \frac{\alpha}{\epsilon}\left(\left\langle 2^{+}\left|\gamma_{\mu}\right| 1^{+}\right\rangle\left\langle 4^{+}\left|\gamma^{\mu}\right| 3^{+}\right\rangle(u-t)-\left\langle 4^{+}\left|\gamma_{\mu}\right| 1^{+}\right\rangle\left\langle 2^{+}\left|\gamma^{\mu}\right| 3^{+}\right\rangle(u-s)\right) \\
=\frac{i \kappa^{4}}{(4 \pi)^{2}} \frac{\alpha}{\epsilon}[24]\langle 31\rangle 2(2 u-t-s) \\
=\frac{i \kappa^{4}}{(4 \pi)^{2}} \frac{6 \alpha}{\epsilon}[42]\langle 13\rangle u=-6 \alpha \frac{i \kappa^{4}}{(4 \pi)^{2}} \frac{u^{3}}{\langle 24\rangle[13]} \frac{1}{\epsilon}
\end{aligned}
$$

(Note that $\left\langle i^{-}\left|\gamma_{\mu}\right| j^{+}\right\rangle=0$, so all other contributions vanish. This also confirms our assertion that any other helicity choice will have no UV divergences at one loop.) Comparing this with (8.48) implies that .

$$
\alpha=-\frac{59}{768}
$$

The fact that this coefficient is non-zero is confirmation that the Dirac-Einstein system is non-renormalisable. Note that we have evaluated part of the counter-Lagrangian which could not be determined from the calculation in ref. [42]. 


\subsection{Gravity Coupled to Photons}

We could take the same route as we have with other particles to find the infinities in photon amplitudes, but we can reduce the work by a judicious use of SUSY identities. We will look at the four point amplitude with no external gravitons. Although our aim is to find the infinities in a system containing photons and gravitons, let us begin by considering the $N=2$ supersymmetric multiplet containing a photon a graviton and two gravitinos. The cut for this amplitude is the sum of the cuts with photons, gravitinos and gravitons in the loop (fig. 8.F)

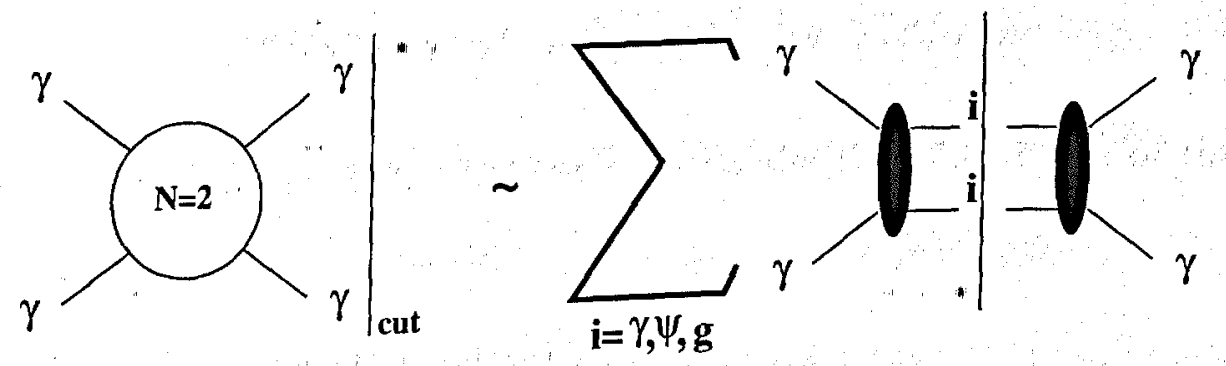

Figure 8.F: Cut contributions required for the one loop

four photon amplitude in an $N=2$ SUGRA theory.

Notice that the tree amplitudes $A^{\text {tree }}(\gamma, \gamma, \gamma, \gamma)$ and $A^{\text {tree }}(g, g, g, g)$ do not include any contribution due to gravitinos (since any internal gravitinos must form a complete loop.) Hence, the only gravitino contribution is contained in the second term in the sum in fig. 8.F. We may deduce that the cuts for the amplitude in which we are interested, that containing only gravitons and photons, are equal to the cuts of the $N=2$ amplitude minus the cut containing gravitinos (fig. 8.G).

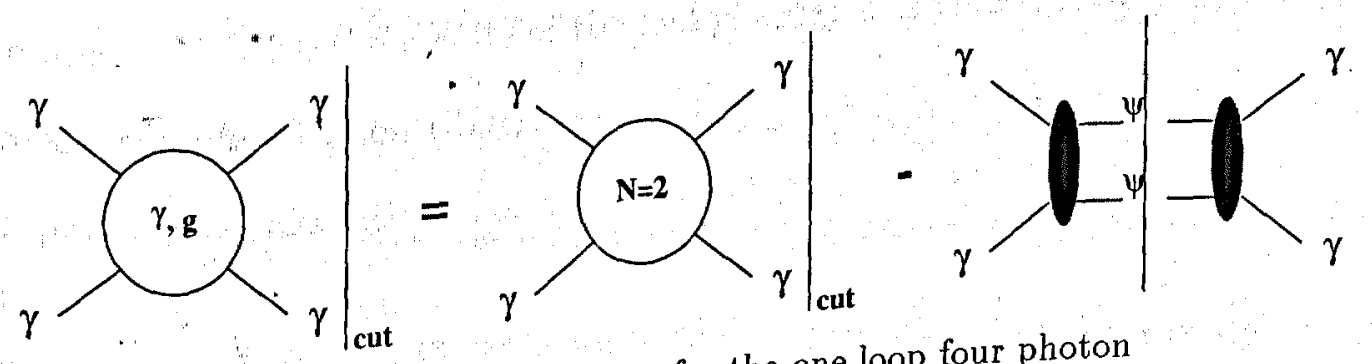

Figure 8.G: Cut equation for the one loop four 
Now, using supersymmetric relations between amplitudes (see Appendix IV) we can make the following deductions: First, for choices of external helicities $\left(\gamma^{-} \gamma^{+} \gamma^{+} \gamma^{+}\right)$and $\left(\gamma^{+} \gamma^{+} \gamma^{+} \gamma^{+}\right)$the gravitino contributions vanish and the cuts will be precisely equal to the cuts in the $N \stackrel{2}{=} 2$ amplitude. We know the $N=2$ amplitude contains only IR divergences and, hence, there will be no UV infinite components in the photon-graviton amplitudes with those helicity choices.

This implies that the amplitude of interest to us here is $A\left(\gamma^{-} \gamma^{+} \gamma^{-} \gamma^{+}\right)$, so let us concentrate on this helicity configuration. SUSY relations give us

$$
A^{\mathrm{N}=2}\left(\gamma^{-} \gamma^{-} \gamma^{+} \gamma^{+}\right)=\frac{\langle 43\rangle^{2}}{\langle 12\rangle^{2}} A^{\mathrm{N}=2}\left(g^{-} g^{-} g^{+} g^{+}\right)
$$

Implying the relation in fig. 8.H. The first term on the right hand side of that relation gives us the cuts

$$
\begin{array}{r}
F^{\prime}\left(\frac{2}{\epsilon}\left(\frac{\ln (-u)}{s t}+\frac{\ln (-t)}{s u}+\frac{\ln (-s)}{t u}\right)\right. \\
+\frac{2 \ln (-u) \ln (-s)}{s u}+\frac{2 \ln (-t) \ln (-u)}{t u}+\frac{2 \ln (-t) \ln (-s)}{t s} \\
+\frac{\left(3 t^{4}+3 t^{3} u-t^{2} u^{2}+3 t u^{3}+3 u^{4}\right)\left(\ln ^{2}(-t /-u)\right)}{s^{6}} \\
\left.+\frac{(t-u)\left(26 t^{2}+46 t u+26 u^{2}\right) \ln (-t /-u)}{s^{5}}\right)
\end{array}
$$

where $F^{\prime}$ here is

$$
\frac{i \kappa^{4}(4 \pi)^{\epsilon} r_{\Gamma}}{16(4 \pi)^{2}} \frac{\langle 43\rangle^{2}}{\langle 12\rangle^{2}}\left(\frac{s t\langle 12\rangle^{4}}{\langle 12\rangle\langle 23\rangle\langle 34\rangle\langle 41\rangle}\right)^{2}=\frac{s t u \kappa^{2}(4 \pi)^{\epsilon} r_{\Gamma}}{4(4 \pi)^{2}} A^{\text {tree }}\left(\gamma^{-}, \gamma^{-}, \gamma^{+}, \gamma^{+}\right)
$$

If we compare this with the results of the last chapter it is clear that the divergences seen here are the complete IR contributions expected; there are no UV contributions from this ${ }^{\prime} N=2$, part of the calculation. So, the UV divergence we are looking for will be found in the subtracted gravitino contribution.

In fact, we only need to carry out one calculation to find the divergences in the gravitino contribution. Note that, if the helicities on one side of the cut are the same 


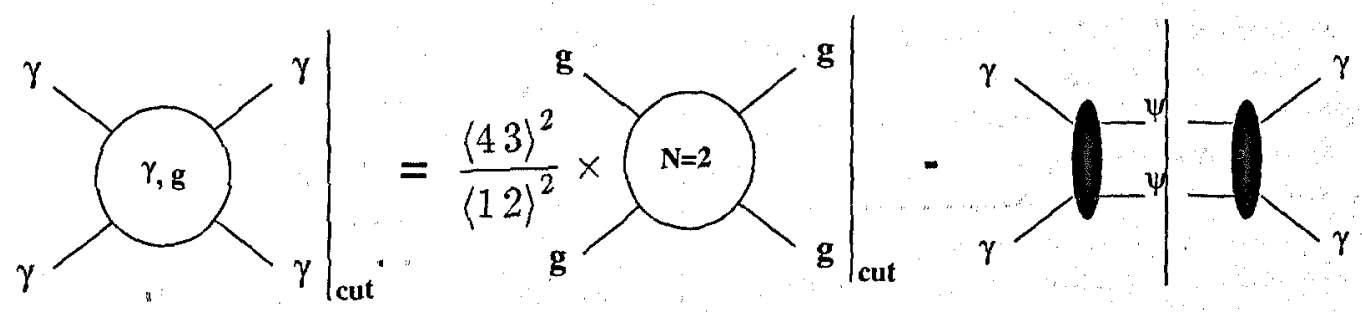

Figure 8.H: Cut equation for the one loop four photon amplitude in Einstein-Maxwell theory (rewritten).

then at least one tree will vanish. Also, there is a symmetry between the two non-zero cut contributions - those in the $t$ - and $u$-channels. "So, if we calculate the $t$-channels divergences, we will have the complete result. Using a combination of SUSY and previous results, we have reduced our work to one calculation.

Let us look at the $t$-channel contribution. For this we will need the photon-gravitino tree. This can be found via another supersymmetric relation:

$$
A^{\mathrm{N}=2}\left(\gamma^{-} \psi^{-} \gamma^{+} \psi^{+}\right)=\frac{\langle 32\rangle\langle 34\rangle}{\langle 12\rangle^{2}} A^{\mathrm{N}=2}\left(g^{-} g^{-} g^{+} g^{+}\right)
$$

So, we require the $d$ LIPS integral of

$$
\begin{aligned}
A\left(\gamma_{1}^{-} \dot{\psi}_{l_{1}}^{-} \gamma_{4}^{+} \psi_{l_{2}}^{+}\right) A\left(\gamma_{2}^{-} \psi_{l_{2}}^{-} \gamma_{3}^{+} \psi_{l_{1}}^{+}\right) & =-\frac{\kappa^{4}}{16} \frac{\left(4 . l_{2}\right)^{2}\left\langle 1 l_{1}\right\rangle^{3}}{\left\langle l_{2} 4\right\rangle^{2}\left\langle 1 l_{2}\right\rangle(1.4)} \times \frac{\left(3 . l_{1}\right)^{2}\left\langle 2 l_{2}\right\rangle^{3}}{\left\langle l_{1} 3\right\rangle^{2}\left\langle 2 l_{1}\right\rangle(2.3)} \\
& =-\frac{\kappa^{4}}{16 t^{2}} \frac{\operatorname{tr}_{+}\left(4 l_{2} 21 l_{1} 3\right)^{2} \operatorname{tr}_{+}\left(1 l_{1} 2 l_{2}\right)}{\langle 21\rangle^{2}[34]^{2}\left(1 . l_{2}\right)\left(2 . l_{1}\right)}
\end{aligned}
$$

We can deal with the traces in a similar way to the scalar case:

$$
\begin{aligned}
\operatorname{tr}_{+}\left(1 l_{1} 2 l_{2}\right) & =\operatorname{tr}_{+}\left(1 l_{1} 2 l_{1}\right)+\operatorname{tr}_{+}\left(1 l_{1} 23\right) \\
& =4\left(1 . l_{1}\right)\left(2 l_{1}\right)+\operatorname{tr}_{+}\left(1 l_{1} 23\right) \\
& =4\left(1 . l_{1}\right)\left(2 . l_{1}\right)+\frac{1}{2} \operatorname{tr}\left(1 l_{1} 23\right)+\frac{1}{2} \epsilon\left(1 l_{1} 23\right)
\end{aligned}
$$

and

$$
\begin{aligned}
\operatorname{tr}_{+}\left(4 l_{2} 21 l_{1} 3\right)= & \operatorname{tr}_{+}\left(4 l_{1} 21 l_{1} 3\right)+\operatorname{tr}_{+}\left(4321 l_{1} 3\right) \\
= & 2\left(l_{1} .2\right) \operatorname{tr}+\left(41 l_{1} 3\right)-2\left(l_{1} \cdot 1\right) \operatorname{tr}+\left(42 l_{1} 3\right)+2 \operatorname{tr}_{+}\left(21 l_{1} 3\right)(4.3) \\
= & \left(l_{1} .2\right)\left(\operatorname{tr}\left(41 l_{1} 3\right)+\epsilon\left(41 l_{1} 3\right)\right) \\
& \quad-\left(l_{1} .1\right)\left(\operatorname{tr}\left(42 l_{1} 3\right)+\epsilon\left(42 l_{1} 3\right)\right)+(4.3)\left(\operatorname{tr}\left(21 l_{1} 3\right)+\epsilon\left(21 l_{1} 3\right)\right)
\end{aligned}
$$




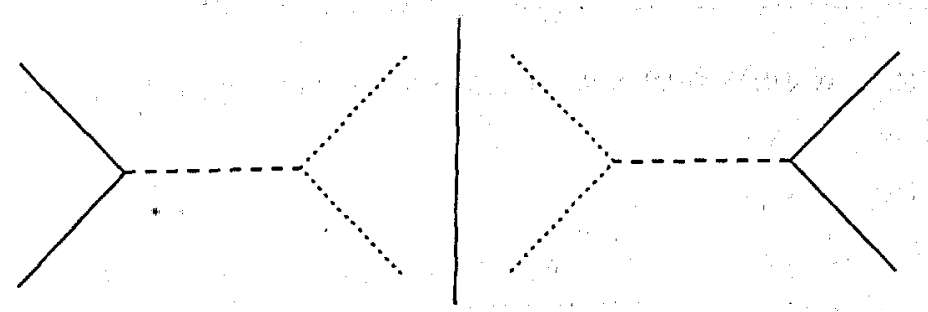

Figure 8.I: Extra cut required when a second photon flavour is added to the theory.

As before, we need only take into account terms with an even number of epsilon tensors; pairs of these tensors can be expanded using the relations in appendix VI to give us an expression which we can integrate in the usual way. When this is done, we get cut contributions

$$
\frac{i \kappa^{4} r_{\Gamma}}{(4 \pi)^{2-\epsilon}} \frac{s^{4}}{\langle 21\rangle^{2}[34]^{2}}\left(\frac{137}{960} \ln (t)+\frac{137}{960} \ln (u)-\ln ^{2}(t / u)\right) .
$$

We must double this result since there are two gravitinos in the SUSY multiplet; the contributions from both should be subtracted. So, we deduce that the UV divergence is

$$
\frac{137}{240} \frac{i \kappa^{4}}{(4 \pi)^{2}} \frac{1}{\epsilon} \frac{s^{4}}{(21)^{2}[34]^{2}}
$$

As with the scalar case, we can compare this with the theoretical derivation of the counterterms (see ref. $[71]^{\dagger}$ ), We can confirm that these terms do, indeed, cancel this, as required.

We can also look at the effect of adding more (independent) $U(1)$ particles to the system. To add $n$ more photons, we must consider $n$ diagrams of the form fig. 8.I, where the internal particle is a photon, but a different flavour to the external one. This requires us to evaluate the cut expression

$$
\frac{\kappa^{4}}{16 t^{2}} \frac{t r_{+}\left(4 l_{2} 21 l_{1} 3\right)^{2}}{\langle 21\rangle^{2}[34]^{2}}
$$
This actually differs from the result in ref. [71] by a factor of 4 . This is simply due to a difference 
We require one copy of this for each extra photon included in our analysis. If we carry out the usual analysis, and sum over all channels, we find that $n$ extra photons produce an added UV infinite contribution of

$$
\frac{n}{40} \frac{i \kappa^{4}}{(4 \pi)^{2}} \frac{1}{\epsilon} \frac{s^{4}}{\langle 21\rangle^{2}[34]^{2}}
$$

Again we can compare this with the theoretical results [72] and find that the derived counterterms will remove this divergence.

So, we have been able to use the cuts to find useful information in situations where we could not obtain complete amplitudes. In particular, we have been able to confirm theoretical derivations of divergences in theories with scalars and photons coupled to gravity. We have also been able to determine counterterm coefficients for the Fermion-Einstein system which have not be found before.

All of these calculations have worked with the fact that we can completely determine terms an order of $\epsilon^{-1}$ higher than the order at which we are working. In the next chapter we will show how this can be used to obtain complete amplitudes. 
When you expect nothing and get everything, that's destiny.

Damian Chapa in 'Bound by Honor'

\section{Cutkosky Rules III: Complete Amplitudes}

In the previous section we saw that there are certain parts of any amplitude for which we can find complete expressions. In particular, if, we are working at order $\epsilon^{0}$, then we can find the total coefficient of $\epsilon^{-1}$. It is clear from looking at the basis of functions which we use to construct the amplitudes that this can be generalised to all orders in $\epsilon$. That is, if we find a cut expression at order $\epsilon^{n}$ then we can find exactly the coefficient of $\epsilon^{n-1}$. The next step is to consider whether we can use this to help us find complete results for any amplitude, even those which do not fulfil the conditions of the uniqueness theorem. In fact, we can. The Cutkosky rules are not dependent on the dimension in which we work, so we can try to use these rules in $4-2 \epsilon$ dimensions. This will give us the cuts correctly to all orders in $\epsilon$ and so will also give us the complete amplitude to all orders as well. At one loop there is only one part of the process which depends on the dimensions - the integral. So, we expect that we can find complete amplitudes by following a similar path to earlier derivations, but with integrals carried out in $4-2 \epsilon$ dimensions.

This method of finding complete amplitudes was originally demonstrated by Bern and Morgan; in ref. [18] they were able to find four gluon amplitudes which were not cutconstructible by the earlier criteria. In addition they were able to use the method to obtain four gluon amplitudes with internal massive particles. In this chapter we follow a similar path for graviton amplitudes. We will see that, while the process allows us to deduce far more information than other cut calculations we have considered, it is not significantly more complicated. 


\subsection{Cut Calculation of $A^{1-\mathrm{loop}}(+,+,+,+)$}

Let us look at the way this combination of dimensional regularisation and Cutkosky rules can be used to find one of the amplitudes which we were able to calculate using the string rules, but which was not recoverable using the earlier cut calculations. We will consider the four graviton amplitude $A^{1-\mathrm{loop}}(+,+,+,+)$. Recall that this could not be calculated using Cutkosky methods before because, for all internal particles, at least one of the trees vanished, giving no cut contribution at $\mathcal{O}(\epsilon)$. Here we will consider that case with internal scalars. We know from SUSY decomposition that the result is the same for each type of internal particle, so the scalar calculation will be sufficient to find the complete result."

Let us begin by considering the new technologies required for this calculation. As we have remarked, the difference which we must introduce is to carry out our integral in $D=4-2 \epsilon$ dimensions. In practice, we do this by the following method (see, for instance, ref. [8]): We begin by splitting the momentum vector into to two parts

$$
p_{D}=p_{4}+\mu
$$

where $p_{4}$ is a four-dimensional part, only the usual 4 space-time components are non-zero, and $\mu^{\alpha} \mathrm{a}$ ' $-2 \epsilon^{\prime}$ dimensional part, only the extra $-2 \epsilon$ components are non-zero. $\mu^{\alpha}$ is made up from the space-like components of $p_{D}$ only, and we explicitly chose to work with a Minkowski metric of negative signature. So $p_{D}^{2}$ can be expressed as

$$
p_{D}^{2}=p_{4}^{2}-\mu^{2}
$$

It is clear that this additional piece now looks, like a mass, and indeed, single powers of $\mu^{\alpha}$ do not appear in our calculations, so we can consistently treat it this way. The subtlety is that the total $\mathrm{D}$-dimensional vector is integrated over, so there must be some integration over this mass-like piece. To do this we begin by splitting up the integral as

$$
\int \frac{d^{D} p}{(2 \pi)^{D}} \rightarrow \int \frac{d^{4} p}{(2 \pi)^{4}} \frac{d^{-2 \epsilon} \mu}{(2 \pi)^{-2 \epsilon}}
$$


We can then deal with the $\int d^{-2 \epsilon} \mu$ by considering changing to spherically symmetric coordinates in our $(-2 \epsilon)$-dimensional subspace. Since the integrand is only dependent on $\mu^{2}$, we do not need to worry about angular dependence and can integrate this out explicitly. The general expression for a solid angle in $(d+1)$-dimensions is

$$
\int d \Omega_{d}=\frac{2 \pi^{(d+1) / 2}}{\Gamma\left(\frac{d+1}{2}\right)}
$$

So, we find

$$
\begin{aligned}
\int \frac{d^{-2 \epsilon} \mu}{(2 \pi)^{-2 \epsilon}} f\left(\mu^{2}\right) & =\int d \Omega_{-1-2 \epsilon} \int d \mu^{2} \frac{1}{2}\left(\mu^{2}\right)^{-1-\epsilon} f\left(\mu^{2}\right) \\
& =\frac{-\epsilon(4 \pi)^{\epsilon}}{\Gamma(1-\epsilon)} \int_{0}^{\infty} d \mu^{2}\left(\mu^{2}\right)^{-1-\epsilon} f\left(\mu^{2}\right)
\end{aligned}
$$

This process allows us to deal with our D-dimensional integral in a fairly straightforward way - the extra dimensions can be made to look like a mass which will be integrated over, with a particular measure, to give the required result.

The only other subtlety which we must consider is the expression for the trees. The trees required are $A^{\text {tree }}\left(i^{+}, j^{+}, \phi, \phi\right)$. These vanished for a massless scalar, however our splitting of the $D$-dimensional momentum means that we must consider the expression for a tree with scalar of mass $\mu$. To simplify our calculations we would like to use the spinor helicity technique as before. The problem here is that this is constructed for use with on-shell, massless particles. To get around this restriction we can write the momenta of the scalars as a sum of two null four vectors [88]:

$$
p_{s 1}=q_{1}+\alpha q_{2}, \quad p_{s 2}=\alpha q_{1}+q_{2}
$$

with the condition

$$
p_{s 1}^{2}=p_{s 2}^{2}=\mu^{2}=2 \alpha q_{1} \cdot q_{2}
$$

This expresses the system as four null vectors, which allows us to implement the spinor helicity method in the usual way. We simply convert the resulting expression by using the above relations at the end of our calculation. We find the result

$$
A_{4}\left(1^{s}, 2^{+}, 3^{+}, 4^{s}\right)=-\frac{i \kappa^{2}}{4} \frac{\left(\mu^{4}\right)[23]^{2}}{\langle 23\rangle^{2}}\left(\frac{1}{\left(k_{1}+k_{2}\right)^{2}-\mu^{2}}+\frac{1}{\left(k_{1}+k_{3}\right)^{2}-\mu^{2}}\right)
$$


(Clearly, this vanishes for $\mu=0$, as expected.)

We now have reduced the ' $4-2 \epsilon$ dimensional' calculation to something which looks very similar to our earlier ' 4 dimensional' calculations. The only subtlety which we have is the extra parameter $\mu^{2}$ which must be integrated out.

Since we are considering the $A^{1-\mathrm{loop}}(+,+,+,+)$ amplitude we need only consider one channel - all others will be the equivalent by symmetry. Let us look at the $s$-channel. As with earlier calculations, we sew together two trees

$$
\frac{i}{2} \int d^{D} \operatorname{LIPS}\left(-\ell_{1}, \ell_{2}\right) A^{\text {tree }}\left(-\ell_{1}, 1^{+}, 2^{+}, \ell_{2}\right) A^{\text {tree }}\left(-\ell_{2}, 3^{+}, 4^{+}, \ell_{1}\right)
$$

Again, rather than a direct calculation, we look at an integral which we know will give us the correct cut contribution

$$
-\left.\int \frac{d^{D} \ell_{1}}{(2 \pi)^{D}} A^{\text {tree }}\left(-\ell_{1}, 1^{+}, 2^{+}, \ell_{2}\right) \frac{1}{\ell_{2}^{2}} A^{\text {tree }}\left(-\ell_{2}, 3^{+}, 4^{+}, \ell_{1}\right) \frac{1}{\ell_{1}^{2}}\right|_{\text {cut }}
$$

If we carry out the above rewriting of the integral, this now becomes

$$
\begin{aligned}
& -\int \frac{d^{4} \ell_{1}}{(2 \pi)^{4}} \frac{-\epsilon(4 \pi)^{\epsilon}}{\Gamma(1-\epsilon)} \int_{0}^{\infty} d \mu^{2}\left(\ddot{\mu}^{2}\right)^{-1-\epsilon} \\
& \left.A^{\text {tree }}\left(-\ell_{1}, 1^{+}, 2^{+}, \ell_{2}\right) \frac{1}{\ell_{2}^{2}-\mu^{2}} A^{\text {tree }\left(--\ell_{2}, 3^{+}, 4^{+}, \ell_{1}\right) \frac{1}{\ell_{1}^{2}-\mu^{2}}}\right|_{\text {cut }}
\end{aligned}
$$

Inserting the expression for the trees (9.8), we find two box contributions - corresponding to boxes with external leg ordering 1234 and 1243 . Consider the first of these; we get an integral expression

$$
\begin{array}{r}
-\frac{i \kappa^{4}}{16} \frac{\epsilon(4 \pi)^{\epsilon}}{\Gamma(1-\epsilon)} \frac{[12]^{2}[34]^{2}}{\langle 12\rangle^{2}\langle 34\rangle^{2}} \int \frac{d^{4} p}{(2 \pi)^{4}} \int_{0}^{\infty} d \mu^{2}\left(\mu^{2}\right)^{-1-\epsilon} \mu^{8} \times \frac{1}{\left(p^{2}-\mu^{2}\right)} \\
\times \frac{1}{\left(\left(p-k_{1}\right)^{2}-\mu^{2}\right)} \times \frac{1}{\left(\left(p-k_{1}-k_{2}\right)^{2}-\mu^{2}\right)} \times \frac{1}{\left(\left(p-k_{1}-k_{2}-k_{3}\right)^{2}-\mu^{2}\right)}
\end{array}
$$

After carrying out a Feynman parameterisation, we can rewrite this as

$$
\begin{aligned}
-\frac{i \kappa^{4}}{16} \frac{\epsilon(4 \pi)^{\epsilon}}{\Gamma(1-\epsilon)} \frac{[12]^{2}[34]^{2}}{\langle 12\rangle^{2}\langle 34\rangle^{2}} \int \prod_{i=1}^{4} d a_{i} \delta\left(\sum_{j=1}^{4} a_{j}-1\right) \\
\times \int_{0}^{\infty} d \mu^{2}\left(\mu^{2}\right)^{-1-\epsilon} \frac{\mu^{8}}{\left(s a_{1} a_{3}+t a_{2} a_{4}-\mu^{2}\right)^{2}}
\end{aligned}
$$


At this point we may carry out the 'mass' integration

$$
\begin{aligned}
\int_{0}^{\infty} d \mu^{2}\left(\mu^{2}\right)^{-1-\epsilon} \frac{\mu^{8}}{\left(s a_{1} a_{3}+t a_{2} a_{4}-\mu^{2}\right)^{2}} & =\mathrm{B}(4-\epsilon,-4+\epsilon)\left(-s a_{1} a_{3}-t a_{2} a_{4}\right)^{2-\epsilon} \\
& =\frac{\pi(3-\epsilon)}{\sin (\pi \epsilon)}\left(-s a_{1} a_{3}-t a_{2} a_{4}\right)^{2-\epsilon}
\end{aligned}
$$

( $\mathrm{B}(x, y)$ is the conventional Beta-function), allowing us to write the $s$-channel cut (including both box orderings) as

$$
-\frac{i \kappa^{4}}{16(4 \pi)^{2-\epsilon}} \frac{[12]^{2}[34]^{2}}{\langle 12\rangle^{2}\langle 34\rangle^{2}} \frac{\pi \epsilon(3-\epsilon)}{\sin (\pi \epsilon)} \frac{\epsilon(1-\epsilon)(2-\epsilon)}{\Gamma(1-\epsilon) \Gamma(1+\epsilon)}\left(I_{1234}^{D=12-2 \epsilon}+I_{1243}^{D=12-2 \epsilon}\right)
$$

where $I_{i j k l}^{D}$ indicates the four point box integral in D-dimensions with external legs ordered $i j k l$ :

$$
I_{i j k l}^{D} \equiv \Gamma(4-D / 2) \int d a_{i} \delta\left(\sum a_{i}-1\right) \frac{1}{\left(-s_{i j} a_{1} a_{3}-s_{j k} a_{2} a_{4}\right)^{4-D / 2}}
$$

Note that (9.15) is valid to all orders in $\epsilon$. An examination of the coefficient of the integrals in (9.15) shows that we have an overall $\epsilon$ there so, since we only require the loop amplitude to $\mathcal{O}\left(\epsilon^{0}\right)$, we need only evaluate the divergent parts of $I^{D=12-2 \epsilon}$. This can be done very easily since it is the same form as the integrals we required in earlier chapters. We find

$$
\begin{aligned}
I^{D=12-2 \epsilon} & =\Gamma(-2+\epsilon) \int d a_{i} \delta\left(\sum a_{i}-1\right)\left(-s a_{1} a_{3}-t a_{2} a_{4}\right)^{2-\epsilon} \\
& =\frac{1}{2 \epsilon} \int d a_{i} \delta\left(\sum a_{i}-1\right)\left(-s a_{1} a_{3}-t a_{2} a_{4}\right)^{2}+\mathcal{O}\left(\epsilon^{0}\right) \\
& =\frac{2 s^{2}+s t+2 t^{2}}{5040 \epsilon}+\mathcal{O}\left(\epsilon^{0}\right)
\end{aligned}
$$

Inserting this into (9.15) and summing over channels, as usual, we obtain the total expression

$$
-\frac{i \kappa^{4}}{(4 \pi)^{2}}\left(\frac{s t}{\langle 12\rangle\langle 23\rangle\langle 34\rangle\langle 41\rangle}\right)^{2} \frac{\left(s^{2}+t^{2}+u^{2}\right)}{3840}
$$

We can see that this confirms our earlier calculation using the string-inspired techniques $(5.47)$.

So, it is clear that we can use the cut technique in this way for any amplitude and not just the 'cut-constructible' ones identified earlier. Surprisingly, the method is not significantly more complex than the earlier cut calculations; all we require is a wider knowledge of integrals and trees which include massive particles. 


\subsection{Loops with Internal Massive Particles}

In the previous section we calculated one loop results by introducing what might be regarded, in some senses, as a fictitious mass. We might wonder whether, since we can do such calculations with comparative ease, it would be possible to do a similar calculation for internal particle with a real mass. Up to now all our results have been restricted to massless theories so this would be a useful extension. We will see that a calculation with a massive scalar is well within our reach.

Let us consider the amplitude above, but with the internal scalar having a mass $m$. We can do this very easily by substituting all occurrences of $\mu^{2}$ with $\mu^{2}+m^{2}$ in our initial expressions. The $\mu^{8}$ term in the numerator will become $\left(\mu^{2}+m^{2}\right)^{4}$, so we will have a series of terms with differing powers of $\mu^{2}$. There will also be changes in the denominator, changing the $\mu$ integration (9.14). The general integral which we must, consider now becomes

$$
\begin{aligned}
\int d a_{i} \delta\left(\sum a_{i}-1\right) \int_{0}^{\infty} d \mu^{2}\left(\mu^{2}\right)^{-1-\epsilon} \frac{\mu^{2 n}}{\left(s a_{1} a_{3}+t a_{2} a_{4}-\mu^{2}-m^{2}\right)^{2}} \\
=\mathrm{B}(n-\epsilon,-n+\epsilon) \int d a_{i} \delta\left(\sum a_{i}-1\right)\left(-s a_{1} a_{3}-t a_{2} a_{4}+m^{2}\right)^{n-\epsilon-2} \\
=\frac{(-1)^{n} \pi(n-\epsilon-1)}{\sin (\pi \epsilon)} \frac{1}{\Gamma(2+\epsilon-n)} I_{1234}^{D=2 n-2 \epsilon+4}(m)
\end{aligned}
$$

If we expand the integral and sum over the 3 channels then we find a total contribution which looks like

$$
\begin{aligned}
&-\frac{i \kappa^{4}}{16(4 \pi)^{2-\epsilon}} \frac{[12]^{2}[34]^{2}}{\langle 12\rangle^{2}\langle 34\rangle^{2}} \frac{\pi \epsilon}{\sin (\pi \epsilon) \Gamma(1-\epsilon) \Gamma(1+\epsilon)} \\
& \times\left(\epsilon(1-\epsilon)(2-\epsilon)(3-\epsilon) I_{1234}^{D=12-2 \epsilon}(m)\right. \\
&+4 m^{2} \epsilon(1-\epsilon)(2-\epsilon) I_{1234}^{D=10-2 \epsilon}(m)+6 m^{4} \epsilon(1-\epsilon) I_{1234}^{D=8-2 \epsilon}(m) \\
&\left.+4 m^{6} \epsilon I_{1234}^{D=6-2 \epsilon}(m)-m^{8} I_{1234}^{D=4-2 \epsilon}(m)+\{1243\}+\{1324\}\right)
\end{aligned}
$$

As before, this is valid to all orders in $\epsilon$, but we will restrict ourselves to $\mathcal{O}\left(\epsilon^{0}\right)$. Since $\sin (\pi \epsilon) \sim \pi \epsilon$, the overall coefficient will be $\mathcal{O}\left(\epsilon^{0}\right)$. So, we see that we require $I^{D=12-2 \epsilon}(m)$, $I^{D=10-2 \epsilon}(m), I^{D=8-2 \epsilon}(m)$ and $I^{D=6-2 \epsilon}(m)$ to $\mathcal{O}\left(\epsilon^{-1}\right) ;$ and $I^{D=4-2 \epsilon}(m)$ to $\mathcal{O}\left(\epsilon^{0}\right)$. 
The first of these, $I^{D=12-2 \epsilon}(m)$, evaluates to give

$$
\begin{aligned}
\frac{\epsilon(1-\epsilon)(2-\epsilon)}{\Gamma(1+\epsilon)} I_{1234}^{D=12-2 \epsilon} & =\int d a_{i} \delta\left(\sum a_{i}-1\right)\left(-s a_{1} a_{3}-t a_{2} a_{4}+m^{2}\right)^{2-\epsilon} \\
& =\frac{2 s^{2}+s t+2 t^{2}}{2520}-\frac{m^{2}(s+t)}{60}+\frac{m^{4}}{6}+\mathcal{O}(\epsilon) .
\end{aligned}
$$

We can find the others by differentiating with respect to $m^{2}$ to give

$$
\begin{aligned}
& \frac{\epsilon(1-\epsilon)}{\Gamma(1+\epsilon)} I_{1234}^{D=10-\epsilon}=-\frac{s+t}{120}+\frac{m^{2}}{6}+\mathcal{O}(\epsilon) \\
& \frac{\epsilon}{\Gamma(1+\epsilon)} I_{1234}^{D=8-2 \epsilon}=1 / 6+\mathcal{O}(\epsilon) \\
& \frac{1}{\Gamma(1+\epsilon)} I_{1234}^{D=6-2 \epsilon}=\mathcal{O}\left(\epsilon^{0}\right) .
\end{aligned}
$$

So, we find an expression for the amplitude ${ }^{\dagger}$

$$
\begin{aligned}
-\frac{i \kappa^{4}}{16(4 \pi)^{2}}\left(\frac{s t}{\langle 12\rangle\langle 23\rangle\langle 34\rangle\langle 41\rangle}\right)^{2}\left(\frac{\left(s^{2}+t^{2}+u^{2}\right)}{240}\right. \\
\left.+\frac{1}{2} m^{4}-m^{8}\left(I_{1234}^{D=4}(m)+I_{1243}^{D=4}(m)+I_{1324}^{D=4}(m)\right)\right)
\end{aligned}
$$

We might expect that this expression for amplitude should also contain terms of the form $\ln \left(m^{2}\right)$, which would not have been recovered in the above analysis. However, we can deduce that no such terms appear by applying the arguments outlined in ref. [18]: $\ln \left(m^{2}\right)$ terms only appear in two integral functions

$$
\begin{array}{r}
I_{1}=m^{2-2 \epsilon} \frac{\Gamma(1+\epsilon)}{\epsilon(\epsilon-1)} \\
I_{2}(0)=m^{-2 \epsilon} \frac{\Gamma(1+\epsilon)}{\epsilon}
\end{array}
$$

$\dagger$ The integral $I_{i j k l}^{D=4}(m)$ has been calculated in ref. [18], and can be expressed as

$$
I_{i j k l}^{D=4}(m)=-\frac{1}{s_{i j} s_{i l}}\left[H\left(-\frac{s_{j l} m^{2}}{s_{i j} s_{i l}}, \frac{m^{2}}{s_{i j}}\right)+H\left(-\frac{s_{j l} m^{2}}{s_{i j} s_{i l}}, \frac{m^{2}}{s_{i l}}\right)\right]+\mathcal{O}(\epsilon)
$$

where

$$
\begin{array}{r}
H(X, Y) \equiv \frac{2}{x_{+}-x_{-}}\left[\ln \left(1-\frac{X}{Y}\right) \ln \left(\frac{-x_{-}}{x_{+}}\right)-\operatorname{Li}_{2}\left(\frac{x_{-}}{y-x_{+}}\right)\right. \\
\left.-\operatorname{Li}_{2}\left(\frac{x_{-}}{x_{-}-y}\right)+\operatorname{Li}_{2}\left(\frac{x_{+}}{x_{+}-y}\right)+\operatorname{Li}_{2}\left(\frac{x_{+}}{y-x_{-}}\right)\right]
\end{array}
$$

$x_{ \pm}=\frac{1}{2}(1 \pm \sqrt{1-4 X}), y=\frac{1}{2}(1+\sqrt{1-4 Y})$ and $\operatorname{Li}_{2}(z)$ is the dilogarithm. 
(In fact, there is a third, $\lim _{k \rightarrow 0} I_{2}^{6-2 \epsilon}\left(k^{2}\right)$, but this can be related to the others by $I_{2}^{6-2 \epsilon}\left(k^{2}\right)=I_{1}+\frac{1}{6} k^{2} I_{2}(0)+\mathcal{O}\left(k^{4}\right)$.) These contain infinities, so we must take them into account if we wish to get the correct divergences in our result. Rather than taking the answer to be just the cut-constructed part, we must look at the general expression

$$
\text { Cut-constructed part }+d_{1} I_{1}+d_{2} I_{2}(0)
$$

We can find $d_{1}$ and $d_{2}$ by choosing values which give the correct infinite terms. Since there are two types of divergence to be considered - quadratic and logarithmic ultraviolet - this will determine the coefficients uniquely. Once we have the divergences correct we know that we will also have the correct $\ln \left(m^{2}\right)$ terms and the complete amplitude.

In the case which we are considering here we know that there should be no UV divergences (using the arguments in chapter 3), and we can confirm that (9.23) already fulfils this condition. So we can deduce that $d_{1}=d_{2}=0$; there are no $\ln \left(m^{2}\right)$ terms.

As a check of our result, we cant make a large $m$ expansion of (9.23): In this limit $I_{1234}^{D=4}$ becomes

$$
\begin{aligned}
\int d a_{i} & \delta\left(\sum a_{i}-1\right)\left(-s a_{1} a_{3}-t a_{2} a_{4}+m^{2}\right)^{-2-\epsilon} \\
= & \int d a_{i} \delta\left(\sum a_{i}-1\right)\left(m^{2}\right)^{-2-\epsilon}\left(1-(2+\epsilon) \frac{S}{m^{2}}+(2+\epsilon)(3+\epsilon) \frac{S^{2}}{2 m^{4}}\right. \\
& -(2+\epsilon)(3+\epsilon)(4+\epsilon) \frac{S^{3}}{6 m^{6}}+(2+\epsilon)(3+\epsilon)(4+\epsilon)(5+\epsilon) \frac{S^{4}}{24 m^{8}} \\
& \left.-(2+\epsilon)(3+\epsilon)(4+\epsilon)(5+\epsilon)(6+\epsilon) \frac{S^{5}}{120 m^{10}} \cdots\right) \\
& =\frac{1}{6 m^{4}}+\frac{s+t}{60 m^{6}}+\frac{2 s^{2}+s t+2 t^{2}}{840 m^{8}}+\frac{3 s^{3}+s t^{2}+s^{2} t+3 t^{3}}{7560 m^{10}}+\mathcal{O}\left(m^{-12}\right) \cdots
\end{aligned}
$$

where $S=-s a_{1} a_{3}-t a_{2} a_{4}$. So, the amplitude becomes

$$
\frac{i \kappa^{4}}{16(4 \pi)^{2}}\left(\frac{s t}{\langle 12\rangle\langle 23\rangle\langle 34\rangle\langle 41\rangle}\right)^{2}\left(\frac{s t u}{504 m^{2}}+\mathcal{O}\left(m^{-4}\right)\right)
$$

This amplitude tends to zero as $m \rightarrow \infty$ as expected. It is also in agreement with $m \rightarrow \infty$ results obtained by other methods [87]. 
Clearly, this implementation of the Cutkosky rules in $4-2 \epsilon$ dimensions is a powerful technique; for a small increase in the complexity of the integral which we must evaluate, we can find complete expressions for any amplitude. Here we have shown how it can be used to reproduce results found using the string-inspired rules and to calculate 4graviton scattering amplitudes with massive particles in the loop. However, this method of reconstructing results is not restricted to amplitudes of a particular kind and we expect that it will be useful in the derivation of a wide range of PQG calculations. 
Think about the future...

Jack Nicholson in 'Batman'

\section{Conclusion and Future Work}

Gauge theories, and particularly quantum gravity, cause significant problems for those wishing to carry out calculations. The conventional method - Feynman diagrams - seems to magnify the problems to the point where a lot of amplitudes are practically unobtainable. In recent years, there have been a number of attempts to find alternatives to Feynman's method. In QCD especially, this has produced new techniques which can be used to determine results in a far more effective way. In this work we have looked at how these new techniques in QCD can be adapted for PQG and SUGRA.

We began by showing how string theory can be used to develop tools for gauge theory calculations. String theories contain gauge theories in their infinite tension limit. By choosing a suitable string and looking at this limit, we can construct an alternative method for finding one-loop results. In the case of PQG, a closed bosonic string is one possible choice. With this starting point we showed how to derive the 'Master Formula', which contains all the amplitude information, and a set of rules for extracting the required results. As presented here, these two components can be used to find any one-loop graviton scattering amplitude. Note that, while the string theory roots of the method remain clear, the formula and rules can be used without reference to their source.

This technique appears to inherit many of the simplifications found in string amplitudes - the number of diagrams which must be considered is significantly less than in Feynman's method and the expressions which must be integrated are particularly compact. This reduces the amount of computation required considerably. We found that calculations are simplified further if we use another method suggested by string theory - the SUSY 
decomposition. This suggests that the most efficient way to calculate a full set of results is to concentrate on SUSY multiplets; contributions for any choice of particles can be found as linear combinations of the SUSY expressions. Using both the string-based rules and the SUSY decomposition we were able to find all one-loop four-graviton amplitudes in PQG.

We have also looked at methods which implement the Cutkosky rules. These take a very different approach to the techniques above: Rather than a direct calculation, Cutkosky-based methods look at the analytic constraints which can be placed on an amplitude. These constraints can then be used to reconstruct some or all of the result. Since it is used at an amplitude level, this method avoids many of the problems encountered when using Feynman diagrams. Using the Cutkosky rules at one-loop, we were able to confirm many of the results found by the string-based method. For the majority, this confirmation was limited to an agreement in the logarithmic parts of the amplitude. However in two cases, we were able to re-derive complete expressions; using a 'uniqueness theorem', we could deduce that the $N=6$ and $N=8$ SUSY matter multiplets are precisely determined by their cuts.

Except for the few special SUSY amplitudes, the naive application of the Cutkosky rules seems to be of limited use. However, there are situations in which the information it provides is valuable. Even when we cannot obtain complete amplitudes we know that the infinite terms we recover will be correct. Using the method for this purpose, we were able to investigate some of the divergences in PQG. It is not possible to distinguish between UV and IR contributions in Cutkosky calculations, but we were able to separate them with an independent investigation of IR infinities. The UV divergences which we found allowed us to confirm previous derivations of the counter-terms in gravity-scalar and gravity-photon systems. In the Dirac-Einstein theory we were able to use our calculation to obtain new information about the counter-Lagrangian.

We can get round the limitations of the Cutkosky-based method if we turn to a more sophisticated approach: Rather than calculating in 4 dimensions, we can work with 
a dimensional regularisation scheme and consider contributions at all orders in $\epsilon$, the regularisation parameter. It is clear from looking at integral functions that knowing cuts to all orders allows us to reconstruct the entire amplitude to all orders, with no ambiguities. Using this method we were able to confirm another of the expressions obtained by the string-based method and find an additional result for gravity coupled to a massive scalar.

So, with this combination of new techniques, we have been able to derive a number of amplitudes in PQG and SUGRA. The majority of these results have not been obtained before. This lack of previous calculations can be attributed to the difficulties involved with conventional methods. Clearly, these new techniques are a powerful extension to our calculational tool-kit and should enable many new quantities to be obtained.

So what of the future? How can this work be extended? Clearly the results we have presented here are only a small subset of the one-loop amplitudes which could be calculated by these methods. Both the string-based methods and the Cutkosky rules can be used for any number of external particles. (Though, of course, there will be an increase in algebraic complexity.) The string-based rules presented here are restricted to external gravitons, but it would be possible (and useful) to look at extensions to other external particles. The Cutkosky rules have no restriction on external particles; the combination of this method with dimensional regularisation provides the means to obtain a wide range of complete results at one-loop.

So, the additional technology required for more one-loop results is minimal. Also useful, but less straightforward, are extensions for higher order calculations. While there has been some work which looks at the string based methods at $n$-loops [89], it is not clear at the present time how a set of rules as useful as those for one-loop could be developed. Another approach to this - the worldline formalism [6] - takes the philosophy of the stringinspired technique and uses it with first quantised particle calculations. This method has been used to reproduce one-loop string-based rules. There has also been some success with 


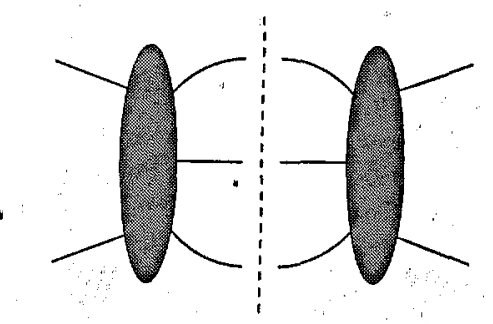

Figure 10.A: Two-loop cut with a three-particle intermediate state

this alternative derivation at two-loops [7] and it is possible that this will provide the best route for $n$-loop extensions. In light of this, an adaptation of the worldline formalism for use in PQG and SUGRA theories would be useful, providing grounding for the exploitation of higher loop methods as they are developed. .

The Cutkosky rules appear to be the most promising path for extensions to higher order in the near future. The method is not dependent in any way on the number of loops in the diagram being considered. The only increase in complexity comes from the number of cuts being considered and the integrals which must be carried out. As an example of the potential of this technique at higher loops, let us consider the 2-loop pure gravity amplitude $A^{2-\operatorname{loop}}(+,+,+,+)$. If we concentrate on the divergence terms in the result and carry out a naive cut calculation in the same way as chapters 6 and 8 , then we find that, for this particular amplitude, we can make a small amount of headway without further technology of any sort [90]:

When applying the Cutkosky rules at two-loops we must consider contributions from threeparticle intermediate states (fig. 10.A), as well' as the two-particles ones found in the oneloop case. (Though for two-loop cuts the latter involves sewing together one one-loop and one tree amplitude, as in fig. 10.B). In general, this will increase the complexity of the

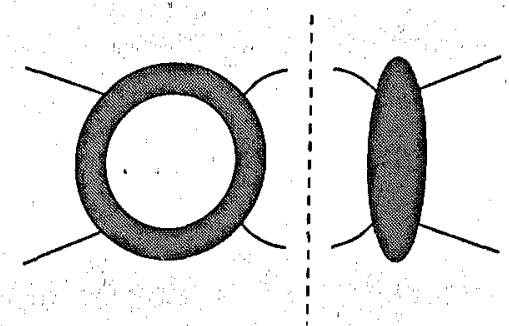

Figure 10.B: Generic two-loop cut with a two-particle intermediate state 

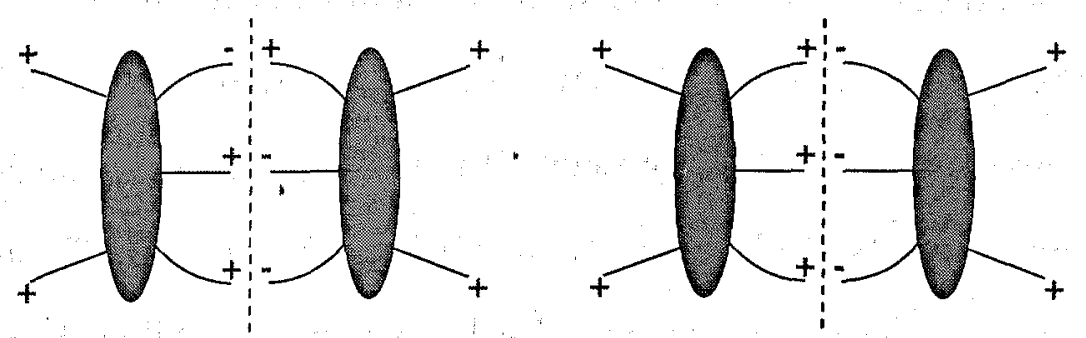

Figure 10.C: Possible helicity configurations of the three-particle cut of $A^{2-\text { loop }}(+,+,+,+)$. In both cases the tree on the

calculation considerably. However, with the helicity configuration which we are considering here we can avoid many of the problems. Note, that the possible helicity configurations for the three particle cuts of this amplitude are in those fig. 10.C. Since we know (from SUSY Ward Identities) that $A^{\text {tree }}(++++-)$ and $A^{\text {tree }}(+++++)$ are both zero, it is clear that these cuts all vanish trivially. So, we are left with two-particle cuts similar to found in one-loop calculations. We can deal with these as before; we simply sew one-loop amplitudes to trees" (fig. 10.D)

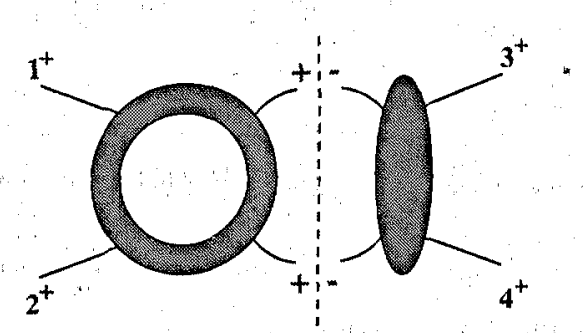

(a)

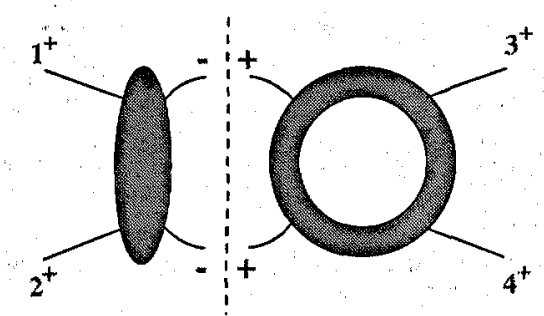

(b)

Figure 10.D: Non-zero contributions to the $s$-channel cut calculation of $A^{2-\operatorname{loop}}(+,+,+,+)$.

If we carry out the calculations using the expressions for one-loop amplitudes in chapter 5 , we find a divergent contribution of

$$
\begin{aligned}
& \sim A^{1-\operatorname{loop}}\left(1^{+}, 2^{+}, 3^{+}, 4^{+}\right) \\
& \quad \times\left(\frac{(-s)^{1-\epsilon}+(-t)^{1-\epsilon}+(-u)^{1-\epsilon}}{2 \epsilon^{2}}+\frac{3\left((-s)^{1-\epsilon}+(-t)^{1-\epsilon}+(-u)^{1-\epsilon}\right)}{2 \epsilon}\right)
\end{aligned}
$$

Referring back to the discussion of soft limits, it is clear that we have the correct IR contribution here. Unfortunately, we can not be so certain that the UV component is 
complete; there are contributions which we have not considered which could be important. For example, the one-loop expressions which we used are only determined to $\mathcal{O}\left(\epsilon^{0}\right)$. If we look at the one-loop integrals, we see that the missing $\mathcal{O}(\epsilon)$ parts of these expressions might also be relevant. To be sure of a full result we should look at Cutkosky calculations in $4-2 \epsilon$ dimensions for all the diagrams. Clearly, this will require a complete set of two-loop integral functions.

While we have only found a small part of the final result in this example, the potential of this approach should be obvious. The restriction has been the difficulty of the integrals rather than problems with the technique Given an improvement in integral technology the Cutkosky-based method will be a useful aid in obtaining higher order amplitude contributions.

In conclusion: We now have a powerful set of new techniques for one-loop amplitude calculations. These methods overcome the complexities which have hampered the traditional Feynman diagram methods and, as a consequence, have allowed us to find a large set of previously unknown results. There is room for optimism foncerning the development of this technology for an increasingly wide range of cases. At one-loop the majority of amplitudes should be calculable using these methods, though further adaptations may be necessary. At higher loops there are still problems which must be overcome; it seems that the most significant impediment at the present time is the lack of necessary integral technology. We may hope that this is a restriction which will be overcome in the near future. 


\section{Appendix I. Quantities in General Relativity}

In this appendix we give general definitions of some of the relevant quantities required in GR and PQG. We also give a brief overview of the expansions used in PQG; that is, when we make small perturbations of the metric tensor (see, e.g., refs. $[28,29,32,56]$ ).

\section{I.1 Definitions}

As usual, we represent the non-covariant derivative by

$$
\begin{aligned}
V^{\alpha_{1} \alpha_{2} \ldots \alpha_{n}} \beta_{\beta_{1} \beta_{2} \ldots \beta_{m}, \nu} & =\partial_{\nu} V^{\alpha_{1} \alpha_{2} \ldots \alpha_{n}}{ }_{\beta_{1} \beta_{2} \ldots \beta_{m}} \\
& =\frac{\partial}{\partial x^{\nu}} V^{\alpha_{1} \ldots \alpha_{n}}{ }_{\beta_{1} \ldots \beta_{m}}
\end{aligned}
$$

and the covariant derivative by

$$
\begin{aligned}
\nabla_{\nu} V_{\beta_{1} \beta_{2} \ldots \beta_{m}}^{\alpha_{1} \alpha_{2} \ldots \alpha_{n}}= & V^{\alpha_{1} \alpha_{2} \ldots \alpha_{n}}{ }_{\beta_{1} \beta_{2} \ldots \beta_{m} ; \nu} \\
= & \frac{\partial}{\partial x^{\nu}} V^{\alpha_{1} \ldots \alpha_{n}}{ }_{\beta_{1} \ldots \beta_{m}} \\
& \quad+\Gamma_{\nu \rho}^{\alpha_{1}} V^{\rho \ldots \alpha_{n}}{ }_{\beta_{1} \ldots \beta_{m}}+\ldots+\Gamma_{\nu \rho}^{\alpha_{n}} V^{\alpha_{1} \ldots \rho}{ }_{\beta_{1} \ldots \beta_{m}} \\
& -\Gamma_{\nu \beta_{1}}^{\rho} V^{\alpha_{1} \ldots \alpha_{n} \ldots \beta_{m}}-\ldots-\Gamma_{\nu \beta_{m}}^{\rho} V^{\alpha_{1} \ldots \alpha_{n}}{ }_{\beta_{1} \ldots \rho} \ldots
\end{aligned}
$$

where the metric connection, $\Gamma_{\beta \gamma}^{\alpha}$, is defined by

$$
\Gamma_{\beta \gamma}^{\alpha}=\frac{1}{2} g^{\alpha \rho}\left(g_{\rho \beta, \gamma}+g_{\rho \gamma, \beta}-g_{\beta \gamma, \rho}\right) .
$$

From this, we also construct the Riemann tensor

$$
R_{\beta \gamma \delta}^{\alpha} \equiv \Gamma_{\beta \delta, \gamma}^{\alpha}-\Gamma_{\beta \gamma, \delta}^{\alpha}+\Gamma_{\rho \gamma}^{\alpha} \Gamma_{\beta \delta}^{\rho}-\Gamma_{\rho \delta}^{\alpha} \Gamma_{\beta \gamma}^{\rho}
$$

The Ricci tensor and scalar are then defined as

$$
\begin{aligned}
R_{\mu \nu} & \equiv R_{\mu \rho \nu}^{\rho} \\
& =\Gamma_{\mu \rho, \nu}^{\rho}-\Gamma_{\mu \nu, \rho}^{\rho}+. \Gamma_{\mu \rho}^{\tau} \Gamma_{\tau \nu}^{\rho}-\Gamma_{\mu \nu}^{\tau} \Gamma_{\tau \rho}^{\rho}
\end{aligned}
$$

and

$$
R \equiv g^{\alpha \beta} R_{\alpha \beta}=g^{\alpha \beta} R_{\alpha \rho \beta}^{\rho}
$$

The Einstein tensor is made up of a linear combination of the Ricci quantities

$$
G_{\alpha \beta} \equiv R_{\alpha \beta}-\frac{1}{2} g_{\alpha \beta} R
$$




\section{I.2 Expansions}

We now give the perturbative expansions of the GR quantities when the metric tensor undergoes small perturbations of the form

$$
g_{\mu \nu}=\tilde{g}_{\mu \nu}+\kappa h_{\mu \nu}
$$

where, $\kappa=\sqrt{8 \pi G}$ and, in all "cases, $\tilde{X}$ will indicate that $X$ depends only on the classical background metric. We use this classical metric to raise and lower the indices on $h_{\mu \nu}$.

We can find the inverse metric tensor, $g^{\mu \nu}$, by requiring that $g_{\mu \alpha} g^{\mu \beta}=\delta_{\alpha}^{\beta}$. . This implies that

$$
g^{\mu \nu}=\tilde{g}^{\mu \nu}-\kappa h^{\mu \nu}+\kappa^{2} h_{\alpha}^{\mu} h^{\alpha \nu}-\kappa^{3} h_{\alpha}^{\mu} h_{\beta}^{\alpha} h^{\beta \nu}+\mathcal{O}\left(\kappa^{4}\right)
$$

We can also find the expansion of $\sqrt{-g}$ from this as follows:

Note first that

$$
\operatorname{det}(X)=\exp (\operatorname{Tr} \ln (X))
$$

and that (I.8) can be rewritten

$$
g_{\mu \nu}=\tilde{g}_{\mu \alpha}\left(\delta_{\nu}^{\alpha}+\kappa h_{\nu}^{\alpha}\right)
$$

Then we can expand it as

$$
\begin{aligned}
\sqrt{-g} & =\sqrt{-\operatorname{det}(g)}=i \exp \left(\frac{1}{2} \operatorname{Tr} \ln (g)\right) \\
& =i \exp \left(\frac{1}{2} \operatorname{Tr} \ln (\tilde{g})+\frac{1}{2} \operatorname{Tr} \ln \left(\delta_{\nu}^{\alpha}+\kappa h_{\nu}^{\alpha}\right)\right) \\
& =\sqrt{-\tilde{g}} \exp \left(\frac{1}{2} \operatorname{Tr}\left(\kappa h_{\nu}^{\alpha}-\frac{1}{2} \kappa^{2} h_{\beta}^{\alpha} h_{\nu}^{\beta}+\frac{1}{3} \kappa^{3} h_{\mu}^{\alpha} h_{\beta}^{\mu} h_{\nu}^{\beta}+\mathcal{O}\left(\kappa^{4}\right)\right)\right) \\
& =\sqrt{-\tilde{g}} \exp \left(\frac{1}{2} \kappa h_{\alpha}^{\alpha}-\frac{1}{4} \kappa^{2} h_{\beta}^{\alpha} h_{\alpha}^{\beta}+\frac{1}{6} \kappa^{3} h_{\mu}^{\alpha} h_{\beta}^{\mu} h_{\alpha}^{\beta}+\mathcal{O}\left(\kappa^{4}\right)\right) \\
& =\sqrt{-\tilde{g}}\left(1+\frac{\kappa}{2} h^{+}+\frac{\kappa^{2}}{8}\left(h^{2}-2 h_{\alpha}^{\beta} h_{\beta}^{\alpha}\right)\right. \\
& \left.\quad+\frac{\kappa^{3}}{48}\left(h^{3}-6 h h_{\alpha}^{\beta} h_{\beta}^{\alpha}+8 h_{\alpha}^{\mu} h_{\beta}^{\alpha} h_{\mu}^{\beta}\right)+\mathcal{O}\left(\kappa^{4}\right)\right)
\end{aligned}
$$


Similarly we can expand the other GR quantities in terms of $h_{\mu \nu}$. First, the Christoffel symbol can be expressed as

$$
\Gamma_{\nu \alpha}^{\mu}=\Gamma_{\nu \alpha}^{(0) \mu}+\Gamma_{\nu \alpha}^{(1) \mu}+\Gamma_{\nu \alpha}^{(2) \mu}+\Gamma_{\nu \alpha}^{(3) \mu}+\mathcal{O}\left(\kappa^{4}\right)
$$

where

$$
\begin{aligned}
& \Gamma_{\nu \alpha \sigma}^{(0) \mu_{\sigma}}=\tilde{\Gamma}_{\nu \alpha}^{\mu} \\
& \Gamma_{\nu \alpha}^{(1) \mu}=\frac{\kappa}{2} \tilde{g}^{\mu \sigma}\left(h_{\sigma \nu ; \alpha}+h_{\sigma \alpha ; \nu}-h_{\alpha \nu ; \sigma}\right) \\
& \Gamma_{\nu \alpha}^{(2) \mu}=\frac{\kappa^{2}}{2} h_{\rho}^{\mu} \tilde{g}^{\rho \sigma}\left(h_{\sigma \nu ; \alpha}+h_{\sigma \alpha ; \nu}-h_{\alpha \nu ; \sigma}\right) \\
& \Gamma_{\nu \alpha}^{(3) \mu}=\frac{\kappa^{3}}{2} h_{\rho}^{\mu} h_{\beta}^{\rho} \tilde{g}^{\beta \sigma}\left(h_{\sigma \nu ; \alpha}+h_{\sigma \alpha ; \nu}-h_{\alpha \nu ; \sigma}\right)
\end{aligned}
$$

From this we find that the Ricci tensor becomes

$$
R_{\mu \nu}=R_{\mu \nu}^{(0)}+R_{\mu \nu}^{(1)}+R_{\mu \nu}^{(2)}+R_{\mu \nu}^{(3)}+\mathcal{O}\left(\kappa^{4}\right)
$$

Where, as before, $R_{\mu \nu}^{(n)} \sim \mathcal{O}\left(\kappa^{n}\right)$. Specifically,

$$
\begin{aligned}
& R_{\mu \nu}^{(0)}=\tilde{R}_{\mu \nu} \\
& R_{\mu \nu}^{(1)}=\Gamma_{\mu \lambda ; \nu}^{(1) \lambda}-\Gamma_{\mu \nu ; \lambda}^{(1) \lambda} \\
& =\frac{\kappa}{2}\left(h_{\beta ; \mu \nu}^{\beta}-h_{\mu ; \nu \alpha}^{\beta}-h_{\nu ; \mu \alpha}^{\beta}+h_{\mu \nu ; \beta}^{\beta}\right) \\
& R_{\mu \nu}^{(2)}=\Gamma^{(2) \lambda}{ }_{\mu \lambda ; \nu}-\Gamma_{\mu \nu ; \lambda}^{(2) \lambda} \text {. } \\
& +\Gamma^{(1) \tau}{ }_{\mu \rho} \Gamma^{(1) \rho}{ }_{\tau \nu}-\Gamma^{(1) \tau}{ }_{\mu \nu} \Gamma^{(1) \rho}{ }_{\tau \rho} \\
& R_{\mu \nu}^{(3)}=\Gamma_{\mu \lambda ; \nu}^{(3) \lambda}-\Gamma_{\mu \nu ; \lambda}^{(3) \lambda} . \\
& +\Gamma_{\mu \rho}^{(1) \tau} \Gamma^{(2) \rho}{ }_{\tau \nu}+\Gamma_{\mu \rho}^{(2) \tau} \Gamma_{\tau \nu}^{(1) \rho} \\
& -\Gamma_{\mu \nu}^{(1) \tau} \Gamma_{\tau \rho}^{(2) \rho}-\Gamma_{\mu \nu}^{(2) \tau} \Gamma_{\tau \rho}^{(1) \rho}
\end{aligned}
$$

And so, the Ricci scalar is

$$
R=R^{(0)}+R^{(1)}+R^{(2)}+R^{(3)}+\mathcal{O}\left(\kappa^{4}\right)
$$


where,

$$
\begin{aligned}
& R^{(0)}=\tilde{R} \\
& R^{(1)}=-\kappa h^{\alpha \beta} \tilde{R}_{\alpha \beta}+\tilde{g}^{\alpha \beta}\left(\Gamma^{(1) \lambda} \dot{\alpha \lambda ; \beta}-\Gamma_{\alpha \beta ; \lambda}^{(1) \lambda}\right) \\
& \quad=\kappa\left(h_{\beta ; \alpha}^{\beta}-h_{\alpha ; \alpha}^{\beta}\right) \\
& \quad R^{(2)}=\tilde{g}^{\alpha \beta} R_{\alpha \beta}^{(2)}-\kappa h^{\alpha \beta} R_{\alpha \beta}^{(1)}+\kappa^{2} h_{\mu}^{\alpha} h^{\mu \beta} \tilde{R}_{\alpha \beta} \\
& R^{(3)}=\tilde{g}^{\alpha \beta} R_{\alpha \beta}^{(3)}-\kappa h^{\alpha \beta} R_{\alpha \beta}^{(2)}+\kappa^{2} h_{\mu}^{\alpha} h^{\mu \beta} R_{\alpha \beta}^{(1)}-\kappa^{3} h_{\alpha}^{\mu} h_{\beta}^{\alpha} h^{\beta \nu} \tilde{R}_{\alpha \beta} .
\end{aligned}
$$




\section{Appendix II. Expansions of Green Functions}

In the derivation of the string-based rules we need expressions for the string Green functions and their derivatives in the limits $\nu \rightarrow 0$ and $\tau, \nu \rightarrow \infty$. Here we give the relevant parts of these expressions (see ref. [3]).

\section{II.1 Open String Green Functions}

As discussed in the text, the basic Green function in the open string case is defined via the two-point correlation function (4.18):

$$
\begin{aligned}
\left\langle X_{\mu}\left(\nu_{i}\right) X_{\nu}\left(\nu_{j}\right)\right\rangle & =\delta_{\mu \nu} G_{B}\left(\nu_{i}-\nu_{j}\right) \\
& =-\delta_{\mu \nu}\left(\log \left|2 \sinh \left(\nu_{i j}\right)\right|-\frac{\left(\nu_{i j}\right)^{2}}{\tau}-4 q \sinh ^{2}\left(\nu_{i j}\right)\right)+\mathcal{O}\left(q^{2}\right)
\end{aligned}
$$

(where $\nu_{i j}=\nu_{i}-\nu_{j}$ )

The infinite tension limit includes the derivatives of this $\dot{G}_{B}(\nu)=\frac{1}{2} \partial_{\nu} G_{B}(\nu)$ and $\ddot{G}_{B}(\nu)=\frac{1}{4} \partial_{\nu}^{2} G_{B}(\nu)$. The second is removed by integration by parts, so we only need limiting expressions for the Green function and its first derivative.

In the limit $\nu \rightarrow 0$, we have

$$
\begin{gathered}
\exp \left(G_{B}(\nu)\right) \sim \nu^{-1 / \pi} \\
\dot{G}_{B}(\nu) \sim \nu^{-1}
\end{gathered}
$$

In the limit $\tau, \nu \rightarrow \infty$

$$
\begin{aligned}
\exp \left(G_{B}(\nu)\right) & \rightarrow \exp \left(\frac{\nu^{2}}{\tau}-|\nu|\right) \times \text { constant } \\
\dot{G}_{B}(\nu) & \rightarrow \frac{\nu}{\tau}-\operatorname{sign}(\nu)\left(\frac{1}{2}+\exp (-2|\nu|)-q \exp (2|\nu|)\right)
\end{aligned}
$$

(recall that $q=\exp (-2 \tau)$ )

\section{II.2 Closed String Green Functions}

In the closed string case the Green function is defined in the same way, but is now a function of a complex variable.

$$
\begin{aligned}
\left\langle X_{\mu}\left(\nu_{i}, \bar{\nu}_{i}\right) X_{\nu}\left(\nu_{j}, \bar{\nu}_{j}\right)\right\rangle & =\delta_{\mu \nu} G_{B}\left(\nu_{i}-\nu_{j}\right) \\
& =-\delta_{\mu \nu}\left(\log \left|2 \sinh \left(\left|\nu_{i j}\right|\right)\right|-\frac{\left(\left|\nu_{i j}\right|\right)^{2}}{\tau}-4 q \sinh ^{2}\left(\left|\nu_{i j}\right|\right)\right)+\mathcal{O}\left(q^{2}\right)
\end{aligned}
$$


(where $\nu_{i j}=\nu_{i}-\nu_{j}$ ). As well as the Green function and first derivatives, $\dot{G}_{B}(\nu)=$ $\frac{1}{2} \partial_{\nu} G_{B}(\nu)$ and $\dot{\bar{G}}_{B}(\bar{\nu})=\frac{1}{4} \partial_{\bar{\nu}} G_{B}(\nu)$ we must also include $H_{B}=\frac{1}{4} \partial_{\nu} \partial_{\bar{\nu}} G_{B}(\nu)$

In the limit $\nu \rightarrow 0$, we have ,

$$
\begin{gathered}
\exp \left(G_{B}(\nu)\right) \sim|\nu|^{-1 / \pi} \\
\dot{G}_{B}(\nu) \sim-\frac{1}{\nu} \quad \dot{\bar{G}}_{B}(\bar{\nu}) \sim-\frac{1}{\bar{\nu}}
\end{gathered}
$$

(We do not require the $\nu \rightarrow 0$ limit of $H_{B}$.) In the limit $\tau, \nu \rightarrow \infty$

$$
\begin{aligned}
\exp \left(G_{B}(\nu)\right) & \rightarrow \exp \left(\frac{(\Im \nu)^{2}}{\Im \tau}-|\Im \nu|\right) \times \text { constant } \\
\dot{G}_{B}(\nu) & \rightarrow \frac{\Im \nu}{\Im \tau}-\operatorname{sign}(\Im \nu)\left(\frac{1}{2}+\exp (-2|\Im \nu|)-q \exp (2|\Im \nu|)\right) \\
\dot{G}_{B}(\bar{\nu}) & \rightarrow \frac{\Im \bar{\nu}}{\Im \tau}-\operatorname{sign}(\Im \bar{\nu})\left(\frac{1}{2}+\exp (-2|\Im \bar{\nu}|)-q \exp (2|\Im \bar{\nu}|)\right)
\end{aligned}
$$

To find $H_{B}$ in this limit we need only consider the first terms in the expansions of $\dot{G}_{B}(\nu)$ and $\dot{G}_{B}(\bar{\nu})$. Noting that $\nu-\bar{\nu}=2 \Im(\nu)$, it is clear that

$$
\partial_{\bar{\nu}} \dot{G}_{B}(\nu)=\partial_{\nu} \dot{G}_{B}(\bar{\nu})=\partial_{\nu}\left(\frac{\bar{\nu}-\nu}{2 \Im \tau}\right)=-\frac{1}{2 \Im \tau}
$$

So,

$$
H_{B}^{i j}=-\frac{1}{2 \Im \tau} \quad \forall i, j
$$

Looking at (II.5) we would expect that there should be a $\delta$-function included in (II.6). Work in ref. [13] implies that this can be ignored for our purposes. We have confirmed this (in calculations of physical amplitudes) with explicit tests: Different choices of the way we integrate by parts will produce different occurrences of $H_{B}$. Any omitted $\delta$-function would be seen in inconsistencies between the results found.

In our description of the string-based rules, we have adjusted the location of some minus signs for the sake of simplicity. Note that we define the variables $x_{i}$ by

$$
x_{i} \equiv \frac{\Im \nu_{i}}{\Im \tau}
$$


This would imply the limit

$$
\begin{aligned}
& \dot{G}_{B}\left(x_{i j}\right) \sim-\frac{1}{2} \operatorname{sign}\left(x_{i j}\right)+x_{i j} \\
& \dot{\bar{G}}_{B}\left(x_{i j}\right) \sim-\left(-\frac{1}{2} \operatorname{sign}\left(x_{i j}\right)+x_{i j}\right) .
\end{aligned}
$$

We have actually chosen to make the two substitutions the same. Changing the sign of the second term requires us to change the $H$ substitution (II.7) to

$$
H_{B}^{i j}=\frac{1}{2 T}
$$

(Each occurrence of an $H$ effectively replaces one $\dot{G}_{B}$ and one $\dot{\bar{G}}_{B}$.) We also require an additional overall $(-1)^{n}$, where $n$ is the number of external legs. 


\section{Appendix III. Spinor-Helicity Techniques}

One major simplification which has been widely used both in QED and QCD calculations and in the work presented here is the spinor-helicity technique. In this method we calculate amplitudes for specific choices of external helicity. Since there is no interference between different helicity configurations, the total cross-section can be found by summing" over all possible helicity amplitudes. Working with specific helicity choices allows significant simplifications to be made in the calculation process. In particular, for external on-shell particles we can use the representation for the helicity vectors introduced by $\mathrm{Xu}$, Zhang and Chang [19,2]. In this representation helicity vectors are written as products of spinors dependent on the particle momentum and an arbitrary reference momentum.

The basic notation which we use is

$$
\begin{aligned}
\langle p+\mid q-\rangle & =[p q] \\
.\langle p-\mid q+\rangle & =\langle p q\rangle
\end{aligned}
$$

with $|q-\rangle$ and $|q+\rangle$ are positive and negative helicity massless Dirac spinors respectively (i.e. $q \cdot \gamma|q \pm\rangle=0$ and $|q \pm\rangle=\frac{1}{2}\left(1 \pm \gamma_{5}\right)|q \pm\rangle$ ). The normalisation is chosen to be such that

$$
\left\langle p \pm\left|\gamma_{\mu}\right| p \pm\right\rangle=2 p_{\mu}
$$

It is possible to show that we can relate vectors and spinors of the same helicity by a linear transformation:

$$
\epsilon_{\mu}^{+}(p) \sim \overline{u_{+}(p)} \gamma_{\mu} v
$$

where $v$ is a second spinor and $\gamma_{\mu}$ are the usual gamma matrices. Further, we can always choose an arbitrary momentum, $\mathrm{k}$, such that $v=|k+\rangle$ (with $k^{2}=0$ and $\left.k \cdot p \neq 0\right)$.

Including suitable normalisations we can define the helicity vectors by

$$
\epsilon_{\mu}^{ \pm}(p, k)= \pm \frac{\left\langle p \pm\left|\gamma_{\mu}\right| k \pm\right\rangle}{\sqrt{2}\langle k \mp \mid p \pm\rangle}
$$


The choice of $k$ is related to the gauge. So, a change of $k$ leads to a gauge transformation

$$
\epsilon_{\mu}^{+}(p ; k)=\epsilon_{\mu}^{+}(p ; q)+\frac{\sqrt{2}\langle q k\rangle}{\langle q p\rangle\langle k p\rangle} p_{\mu} .
$$

Note that this shows clearly an assertion which we make in the text: If we replace an epsilon vector with its equivalent momentum vector, the result will vanish. If this was not true then (III.5) implies that amplitudes will not be gauge invariant.

Having set this representation up, we find that judicious choices of reference momenta can simplify many calculations. If we represent the polarisation vector for a particle of momenta $k_{i}$ with reference momenta $q$, then we find

$$
\begin{aligned}
k_{j} \cdot \epsilon^{+}\left(k_{i} ; q\right) & =\frac{\left\langle q k_{j}\right\rangle\left[k_{j} k_{i}\right]}{\sqrt{2}\left\langle q k_{i}\right\rangle} \quad k_{j} \cdot \epsilon^{-}\left(k_{i} ; q\right)=\frac{\left[q k_{j}\right]\left\langle k_{j} k_{i}\right\rangle}{\sqrt{2}\left[k_{i} q\right]} \\
& \epsilon^{+}\left(k_{j} ; p\right) \cdot \epsilon^{+}\left(k_{i} ; q\right)=\frac{\left[k_{j} k_{i}\right]\langle q p\rangle}{\left\langle k_{j} p\right\rangle\left\langle k_{i} q\right\rangle} \\
& \epsilon^{-}\left(k_{j} ; p\right) \cdot \epsilon^{-}\left(k_{i} ; q\right)=\frac{\left\langle k_{j} k_{i}\right\rangle[q p]}{\left[k_{j} p\right]\left[k_{i} q\right]} \\
& \epsilon^{-}\left(k_{j} ; p\right) \cdot \epsilon^{+}\left(k_{i} ; q\right)=\frac{\left\langle q k_{j}\right\rangle\left[p k_{i}\right]}{\left\langle q k_{i}\right\rangle\left[k_{j} p\right]}
\end{aligned}
$$

From these we can deduce useful identities for specific choices of reference momenta

$$
\begin{array}{r}
\epsilon^{ \pm}\left(k_{j} ; q\right) \cdot \epsilon^{ \pm}\left(k_{i} ; q\right)=0 \\
\epsilon^{ \pm}\left(k_{j} ; q\right) \cdot \epsilon^{\mp}\left(k_{i} ; k_{j}\right)=0 \\
q \cdot \epsilon^{ \pm}\left(k_{j} ; q\right)=0
\end{array}
$$

Also, as required, we confirm that these definitions imply

$$
k_{j} \cdot \epsilon^{ \pm}\left(k_{j} ; q\right)=0
$$

We can also extend the spinor-helicity technique so that it can be used in PQG and SUGRA theories $[20,21,88]$. Gravitons are spin-2 particles, so their helicities are represented by rank 2 tensors rather than vectors. We can, however, make direct use of the above method by noting that the tensors can be written as

$$
\epsilon_{\mu \nu}=\epsilon_{\mu} \bar{\epsilon}_{\nu}
$$


$\epsilon$ and $\bar{\epsilon}$ can be treated independently as helicity vectors during the calculation (in particular, implementing the above techniques) and the full tensor reconstructed at the end [84]. We can take a similar route for gravitinos [8்8].

While these techniques are useful in all amplitude calculations, they are particularly relevant for the techniques we have focussed on. Note that in string calculations all helicity vectors occur in terms like $\epsilon_{i} \cdot \epsilon_{j}$ or $k \cdot \epsilon_{i}$ at the beginning of the calculation. If we use the above, with clever choices for reference momenta, we can significantly reduce the number of terms we must deal with before we start. Also, in the cut techniques the helicity method provides a very compact way of representing the tree amplitudes, simplifying the subsequent work. 


\section{Appendix IV. Supersymmetric Identities}

Supersymmetric theories are constructed with symmetries between particles of different types, so it is not surprising that they generate relations between different amplitudes in the theory. As expected, we can use these symmetries to find a large set of supersymmetric results given a small number of amplitudes [22]. More surprisingly, we also find that it is possible to use these to relate some non-supersymmetric amplitudes [23,2]. Here we show how these relations can be constructed and give the results which we used in our calculations. We begin with a reminder of the essentials of supersymmetric theory.

\section{IV.1 Amplitudes in Supersymmetry}

Supersymmetric theories are theories containing one or more charges, $Q^{i}, \cdot$ which have spin one half. (The number of such charges in a theory is generally denoted by $N$; The most common supersymmetric theories considered are those with $N \leq 8$.) These charges will take fermions to bosons are vice versa. The $Q^{i}$ s transform as spinors under Lorentz transformations, so we can define

$$
Q^{i}(\eta)=\bar{\eta}^{\alpha} Q_{\alpha}^{i}
$$

where $Q_{\alpha}^{i}$ are generators which form a Majorana spinor and $\eta_{\alpha}$ is a fermionic spinor parameter.

If we then look at the case of an $N=1$ supersymmetric multiplet containing a graviton, $\mathrm{g}$, and a gravitino, $\psi$. (This is the example used in ref. [22]. However, here we carry out the analysis using the spinor-helicity formulation as in ref. [2]. This formulation fits more closely with the presentation of our string inspired and Cutkosky results.) The commutation relations of the creation operators with $Q(\eta)$ are

$$
\begin{aligned}
& {\left[Q(\eta), a_{g}^{ \pm}\right]= \pm \Gamma^{ \pm}(p, \eta) a_{\psi}^{ \pm}} \\
& {\left[Q(\eta), a_{\psi}^{ \pm}\right]= \pm \Gamma^{\mp}(p, \eta) a_{g}^{ \pm}}
\end{aligned}
$$


Here $a_{g}^{ \pm}$is the creation operator for a ( $\left.\begin{array}{c}\text { postive } \\ \text { negative }\end{array}\right)$ helicity graviton and $a_{\psi}^{ \pm}$is the creation operator for a ( $\left.\begin{array}{c}\text { postive } \\ \text { negative }\end{array}\right)$ helicity gravitino. As elsewhere, we take all particles to be in-going (so we will not consider equivalent formulae for annihilation operators).

We may define the $\Gamma$ terms in a 'spinor-helicity way' as

$$
\Gamma^{+}(p, \eta)=\bar{\eta} u_{-}(p)
$$

(Note it must be both a spinor and linear in $\eta$.) $\eta$ is completely arbitrary, up to having zero norm, so we can choose it to be the product of a Grassmann parameter, $\theta$, and a positive-helicity spinor of momentum $\mathrm{k}, u_{-}(k)$, implying

$$
\Gamma^{+}(p, \eta)=\overline{\theta u+(k)} u_{-}(p)=\theta[k p]
$$

Similarly, we can put

$$
\Gamma^{-}(p, \eta)=\overline{\theta u_{-}(k)} u_{+}(p)=\theta\langle k p\rangle
$$

Then, with a slight change of notation,

$$
\begin{aligned}
& \Gamma^{+}(p, q)=\theta[k p] \\
& \Gamma^{-}(p, q)=\theta\langle k p\rangle .
\end{aligned}
$$

\section{IV.2 Construction of Identities}

The starting point for using supersymmetry to relate amplitudes is to notice that in an unbroken supersymmetric model the supersymmetric operator annihilates the vacuum,

$$
\begin{aligned}
& Q_{i}|0\rangle=0 \\
& \langle 0| Q_{i}=0
\end{aligned}
$$

where $Q_{i}$ is a supersymmetric operator. Hence, if $\left\{a_{i}\right\}$ is a set of $n$ supersymmetric creation and annihilation operators, then

$$
\left\langle 0\left|\left[Q_{i}, a_{1} a_{2} \ldots a_{n}\right]\right| 0\right\rangle=0
$$


This can easily be expanded to give the Supersymmetric Ward Identity

$$
\sum_{j=1}^{n}\left\langle 0\left|a_{1} \cdots a_{j-1}\left[Q_{i}, a_{j}\right] a_{j+1} \cdots a_{n}\right| 0\right\rangle=0 .
$$

By astute choices of $a_{j}$ 's and use of the above commutation relations it is possible to construct a number of useful relations between various amplitudes.

As an example of the power of these relations, let us consider a number of derivations using the technique with $N=1(g, \psi)$ and $N=2(g, \psi, \gamma)$ supersymmetric multiplets. .

$\mathrm{N}=1$

First, we can easily show that $A\left(g^{+}, g^{+}, g^{+}, g^{+}\right)$and $A\left(g^{-}, g^{+}, g^{+}, g^{+}\right)$are trivial in any supersymmetric multiplet.

From equ. (IV.8) we have

$$
\left\langle 0\left|\left[Q_{i}, a_{\psi}^{+}\left(\bar{k}_{1}\right) a_{g}^{+}\left(k_{2}\right) a_{g}^{+}\left(k_{3}\right) a_{g}^{+}\left(k_{4}\right)\right]\right| 0\right\rangle=0 .
$$

Which gives, expanding as in equ. (IV.9)

$$
\begin{aligned}
& \Gamma^{-}\left(k_{1}, q\right)\left\langle 0\left|a_{g}^{+}\left(k_{1}\right) a_{g}^{+}\left(k_{2}\right) a_{g}^{+}\left(k_{3}\right) a_{g}^{+}\left(k_{4}\right)\right| 0\right\rangle \\
& +\Gamma^{+}\left(k_{2}, q\right)\left\langle 0\left|a_{\psi}^{+}\left(k_{1}\right) a_{\psi}^{+}\left(k_{2}\right) a_{g}^{+}\left(k_{3}\right) a_{g}^{+}\left(k_{4}\right)\right| 0\right\rangle \\
& +\Gamma^{+}\left(k_{3}, q\right)\left\langle 0\left|a_{\psi}^{+}\left(k_{1}\right) a_{g}^{+}\left(k_{2}\right) a_{\psi}^{+}\left(k_{3}\right) a_{g}^{+}\left(k_{4}\right)\right| 0\right\rangle \\
& \quad+\Gamma^{+}\left(k_{4}, q\right)\left\langle 0\left|a_{\psi}^{+}\left(k_{1}\right) a_{g}^{+}\left(k_{2}\right) a_{g}^{+}\left(k_{3}\right) a_{\psi}^{+}\left(k_{4}\right)\right| 0\right\rangle=0
\end{aligned}
$$

The three terms with fermions of the same helicity vanish since interactions with gravity conserve helicity. Hence,

$$
A\left(g^{+}, g^{+}, g^{+}, g^{+}\right)=0
$$

Similarly, consider

$$
\left\langle 0\left|\left[Q_{i}, a_{\psi}^{+}\left(k_{1}\right) a_{g}^{+}\left(k_{2}\right) a_{g}^{+}\left(k_{3}\right) a_{g}^{-}\left(k_{4}\right)\right]\right| 0\right\rangle=0 .
$$


which implies

$$
\begin{aligned}
& \Gamma^{-}\left(k_{1}, q\right)\langle 0| a_{g}^{+}\left(k_{1}\right) a_{g}^{+}\left(k_{2}\right) a_{g}^{+}\left(k_{3}\right) a_{g}^{-}\left(k_{4}\right)|0\rangle \\
&+\Gamma^{+}\left(k_{2}, q\right)\langle 0| a_{\psi}^{+}\left(\dot{k}_{1}\right) a_{\psi}^{+}\left(k_{2}\right) a_{g}^{+}\left(k_{3}\right) a_{g}^{-}\left(k_{4}\right)|0\rangle \\
&+\Gamma^{+}\left(k_{3}, q\right)\left\langle 0\left|a_{\psi}^{+}\left(k_{1}\right) a_{g}^{+}\left(k_{2}\right) a_{\psi}^{+}\left(k_{3}\right) a_{g}^{-}\left(k_{4}\right)\right| 0\right\rangle \\
& \quad-\Gamma^{-}\left(k_{4}, q\right)\left\langle 0\left|a_{\psi}^{+}\left(k_{1}\right) a_{g}^{+}\left(k_{2}\right) a_{g}^{+}\left(k_{3}\right) \dot{a}_{\psi}^{-}\left(k_{4}\right)\right| 0\right\rangle=0 .
\end{aligned}
$$

Again, the middle two terms vanish by helicity conservation. So,

$$
-\Gamma^{-}\left(k_{1}, q\right) A\left(g^{+}, g^{+}, g^{+}, g^{-}\right)-\Gamma^{-}\left(k_{4}, q\right) A\left(\psi^{+}, g^{+}, g^{+}, \psi^{-}\right)=0
$$

If we choose $q=k_{4}$, then $\Gamma^{-}\left(k_{4}, q\right)=0$ and we obtain

$$
A\left(g^{+}, g^{+}, g^{+}, g^{-}\right)=0
$$

(NB. Choosing, instead, $q=k_{1}$ gives us $A\left(g^{+}, g^{+}, \psi^{+}, \psi^{-}\right)=0$.)

As well as identifying trivial amplitudes, the same method gives relations between non-zero amplitudes. As above, we begin by considering

$$
\left\langle 0\left|\left[Q_{i}, a_{\psi}^{+}\left(k_{1}\right) a_{g}^{-}\left(k_{2}\right) a_{g}^{+}\left(k_{3}\right) a_{g}^{-}\left(k_{4}\right)\right]\right| 0\right\rangle=0
$$

implying

$$
\begin{aligned}
& \Gamma^{-}\left(k_{1}, q\right)\left\langle 0\left|a_{g}^{+}\left(k_{1}\right) a_{g}^{-}\left(k_{2}\right) \dot{a}_{g}^{+}\left(k_{3}\right) a_{g}^{-}\left(k_{4}\right)\right| 0\right\rangle \\
& -\Gamma^{-}\left(k_{2}, q\right)\left\langle 0\left|a_{\psi}^{+}\left(k_{1}\right) a_{\psi}^{-}\left(k_{2}\right) a_{g}^{+}\left(k_{3}\right) a_{g}^{-}\left(k_{4}\right)\right| 0\right\rangle \\
& +\Gamma^{+}\left(k_{3}, q\right)\left\langle 0\left|a_{\psi}^{+}\left(k_{1}\right) a_{g}^{-}\left(k_{2}\right) \dot{a}_{\psi}^{-+}\left(k_{3}\right) a_{g}^{-}\left(k_{4}\right)\right| 0\right\rangle \\
& -\Gamma^{-}\left(k_{4}, q\right)\left\langle 0\left|a_{\psi}^{+}\left(k_{1}\right) a_{g}^{-}\left(k_{2}\right) a_{g}^{+}\left(k_{3}\right) a_{\psi}^{-}\left(k_{4}\right)\right| 0\right\rangle=0 .
\end{aligned}
$$

The third term vanishes as before; choosing $q=k_{4}$ and using relation (IV.6) we find

$$
\langle 14\rangle A\left(g^{+}, g^{-}, g^{+}, g^{-}\right)=\langle 24\rangle A\left(\psi^{+}, \psi^{-}, g^{+}, g^{-}\right)
$$


$\mathrm{N}=\mathbf{2}$

If we wish to consider amplitudes with gravitons and particles other than gravitinos then we must use a SUSY multiplet with $N>1$. For instance, let us consider amplitudes with photons and gravitons. For this we need to use the $N=2$ multiplet which is constructed from a graviton, two gravitinos and a photon. First we must note that the $N=2$ Lagrangian is invariant under, the infinitesimal chiral transformation [49]

$$
\delta \psi_{\mu}=i \gamma_{5} \psi_{\mu}: \quad \delta \phi=-i \gamma_{5} \phi_{\mu}
$$

where $\psi_{\mu}$ and $\phi_{\mu}$ are the two gravitino fields.

We deduce that $\psi$ - $\psi$-boson and $\phi$ - $\phi$-boson vertices conserve helicity, whereas a $\psi$ - $\phi$ boson vertex will violate helicity maximally. It then follows that,

$$
\begin{array}{cc}
A\left(\psi^{ \pm}, \psi^{ \pm}, B, B^{\prime}\right)=0 & A\left(\phi^{ \pm}, \phi^{ \pm}, B, B^{\prime}\right)=0 \\
\cdots A\left(\psi^{ \pm}, \phi^{\mp}, B, B^{\prime}\right)=0
\end{array}
$$

where $B$ and $B^{\prime}$ are bosons of either helicity.

We may proceed as before, but must keep watch on which gravitino field is involved in any particular SUSY transformation. (Recall that we now have two SUSY generators.) We work with the following notation:

$$
\begin{array}{ll}
{\left[Q_{1}, a_{g}^{ \pm}\right]= \pm \Gamma^{ \pm}(p, q) a_{\psi}^{ \pm}} & {\left[Q_{2}^{ \pm}, a_{g}^{ \pm}\right]= \pm \Gamma^{ \pm}(p, q) a_{\phi}^{ \pm}} \\
{\left[Q_{1}, a_{\psi}^{ \pm}\right]= \pm \Gamma^{\mp}(p, q) a_{g}^{ \pm}} & {\left[Q_{2}, a_{\phi}^{ \pm}\right]= \pm \Gamma^{\mp}(p, q) a_{g}^{ \pm}} \\
{\left[Q_{2}, a_{\psi}^{ \pm}\right]= \pm \Gamma^{ \pm}(p, q) a_{\gamma}^{ \pm}} & {\left[Q_{1}, a_{\phi}^{ \pm}\right]= \pm \Gamma^{ \pm}(p, q) a_{\gamma}^{ \pm}} \\
{\left[Q_{1}, a_{\gamma}^{ \pm}\right]=\mp \Gamma^{\mp}(p, q) a_{\phi}^{ \pm}} & {\left[Q_{2}, a_{\gamma}^{ \pm}\right]=\mp \Gamma^{\mp}(p, q) a_{\psi}^{ \pm}}
\end{array}
$$

As before we begin with a trivial result $A\left(g^{-}, g^{-}, \gamma^{+}, \gamma^{+}\right)=0$. Our starting point for this is

$$
\left\langle 0\left|\left[Q_{2}, a_{g}^{-}\left(k_{1}\right) a_{\gamma}^{+}\left(k_{2}\right) a_{\psi}^{+}\left(k_{3}\right) a_{g}^{-}\left(k_{4}\right)\right]\right| 0\right\rangle=0
$$


implying,

$$
\begin{aligned}
& \Gamma^{-}\left(k_{1}, q\right)\left\langle 0\left|a_{\phi}^{-}\left(k_{1}\right) a_{\gamma}^{+}\left(k_{2}\right) a_{\psi}^{+}\left(k_{3}\right) a_{g}^{-}\left(k_{4}\right)\right| 0\right\rangle \\
& -\Gamma^{-}\left(k_{2}, q\right)\left\langle 0\left|a_{g}^{-}\left(\dot{k_{1}}\right) a_{\psi}^{+}\left(k_{2}\right) a_{\psi}^{+}\left(k_{3}\right) a_{g}^{-}\left(k_{4}\right)\right| 0\right\rangle \\
& +\Gamma^{+}\left(k_{3}, q\right)\left\langle 0\left|a_{g}^{-}\left(k_{1}\right) a_{\gamma}^{+}\left(k_{2}\right) a_{\gamma}^{+}\left(k_{3}\right) a_{g}^{-}\left(k_{4}\right)\right| 0\right\rangle \\
& \quad-\Gamma^{-}\left(k_{4}, q\right)\left\langle 0\left|a_{\psi}^{+}\left(k_{1}\right) a_{\gamma}^{-}\left(k_{2}\right) a_{\psi}^{+}\left(k_{3}\right) a_{\phi}^{-}\left(k_{4}\right)\right| 0\right\rangle=0 .
\end{aligned}
$$

By the above observation, we can immediately see that the all terms bar the third vanish. This implies that the third term must also by equal to zero, proving the result.

For the Cutkosky calculations, we also require the equivalent relation to (IV.19) for other particle types. Here we look at the result for $A\left(\gamma^{+}, \gamma^{-}, g^{+}, g^{-}\right)$. This amplitude can be found from

$$
\left\langle 0\left|\left[Q_{2}, a_{g}^{-}\left(k_{1}\right) a_{\psi}^{-}\left(k_{2}\right) a_{g}^{+}\left(k_{3}\right) a_{\gamma}^{+}\left(k_{4}\right)\right]\right| 0\right\rangle=0
$$

which expands to give

$$
\begin{aligned}
& \Gamma^{-}\left(k_{1}, q\right)\left\langle 0\left|a_{\phi}^{-}\left(k_{1}\right) a_{\psi}^{-}\left(k_{2}\right) a_{g}^{+}\left(k_{3}\right) a_{\gamma}^{+}\left(k_{4}\right)\right| 0\right\rangle \\
& -\Gamma^{-}\left(k_{2}, q\right)\left\langle 0\left|a_{g}^{-}\left(k_{1}\right) a_{\gamma}^{-}\left(k_{2}\right) a_{g}^{+}\left(k_{3}\right) a_{\gamma}^{+}\left(k_{4}\right)\right| 0\right\rangle \\
& +\Gamma^{+}\left(k_{3}, q\right)\left\langle 0\left|a_{g}^{-}\left(k_{1}\right) a_{\psi}^{-}\left(k_{2}\right) a_{\phi}^{+}\left(k_{3}\right) a_{\gamma}^{+}\left(k_{4}\right)\right| 0\right\rangle \\
& +\Gamma^{-}\left(k_{4}, q\right)\left\langle 0\left|a_{g}^{-}\left(k_{1}\right) a_{\psi}^{-}\left(k_{2}\right) a_{g}^{+}\left(k_{3}\right) a_{\psi}^{+}\left(k_{4}\right)\right| 0\right\rangle=0 .
\end{aligned}
$$

The third term vanishes and we take $q$ to be $k_{1}$, giving

$$
\text { (12) } A\left(g^{-}, \gamma^{-}, g^{+}, \gamma^{+}\right)=\langle 14\rangle A\left(g^{-}, \psi^{-}, g^{+}, \psi^{+}\right)
$$

Which, combined with (IV.19), gives

$$
A\left(g^{-}, \gamma^{-}, g^{+}, \gamma^{+}\right)=\frac{\langle 14\rangle^{2}}{\langle 12\rangle^{2}} A\left(g^{-}, g^{-}, g^{+}, g^{+}\right)
$$

These results can easily be extended to different multiplets to give a wide range of results. 


\section{IV.3 Applications}

There are a number of ways we use these identities in our calculations. First, we note that the results obtained above,

$$
\begin{aligned}
& A^{\text {SUSY }}\left(g^{+}, g^{+}, g^{+}, g^{-}\right)=0 \\
& A^{\text {SUSY }}\left(g^{+}, g^{+}, g^{+}, g^{+}\right)=0
\end{aligned}
$$

are in agreement with our result that, at 1-loop,

$$
\begin{aligned}
& A^{\text {fermion }}\left(g^{+}, g^{+}, g^{+}, g^{-}\right)=-A^{\text {boson }}\left(g^{+}, g^{+}, g^{+}, g^{-}\right) \\
& A^{\text {fermion }}\left(g^{+}, g^{+}, g^{+}, g^{+}\right)=-\dot{A}^{\text {boson }}\left(g^{+}, g^{+}, g^{+}, g^{+}\right)
\end{aligned}
$$

for any boson and fermion in the loop. This can be deduced from the SUSY identities and noting that every multiplet has an equal number of fermions and bosons and that the SUSY amplitudes factor into a sum of amplitudes from individual particles. The latter is because a one-loop amplitude can only include at most one other particle together with the graviton. (This can be seen by looking at the matter-gravity vertices.)

So, for instance, for the $(g, \psi)$ multiplet

$$
\begin{array}{r}
A^{\text {SUSY }}\left(g^{+}, g^{+}, g^{+}, g^{+}\right)=0 \\
\Rightarrow A^{\mathrm{g}}\left(g^{+}, g^{+}, g^{+}, g^{+}\right)+A^{\psi}\left(g^{+}, g^{+}, g^{+}, g^{+}\right)=0
\end{array}
$$

Which generalises if we look at other possible SUSY multiplets. Thus, we have a simple confirmation between theory and calculation.

Second, there is extensive use of these results in the Cutkosky derivation of the oneloop graviton scattering amplitudes. For this we require $A^{\text {tree }}\left(g^{-}, g^{+}, X^{-}, X^{+}\right)$, where $X$ is any particle. By using supersymmetric identities, we can easily see that for any supersymmetric multiplet containing $X$ and $g$

$$
A^{\text {tree }}\left(g^{-}, g^{+}, X^{-}, X^{+}\right)=\frac{\langle 14\rangle^{y}}{\langle 13\rangle^{y}} A^{\text {tree }}\left(g^{-}, g^{+}, g^{-}, g^{+}\right)
$$

(where $\mathrm{y}$ depends on the type of particle $X$ ). 
In the Cutkosky calculations we used this result outside a supersymmetric context. This is justified by the following argument: The minimal coupling of gravity to other fields implies that each vertex containing both gravity and some other field, $\mathrm{X}$, must have at least two X particles. Since only one of these particles can be internal (more than this would imply a loop in the diagram), the only possible contributions to $A^{\text {tree }}(g, g, X, X)$ are those in fig. IV.A.
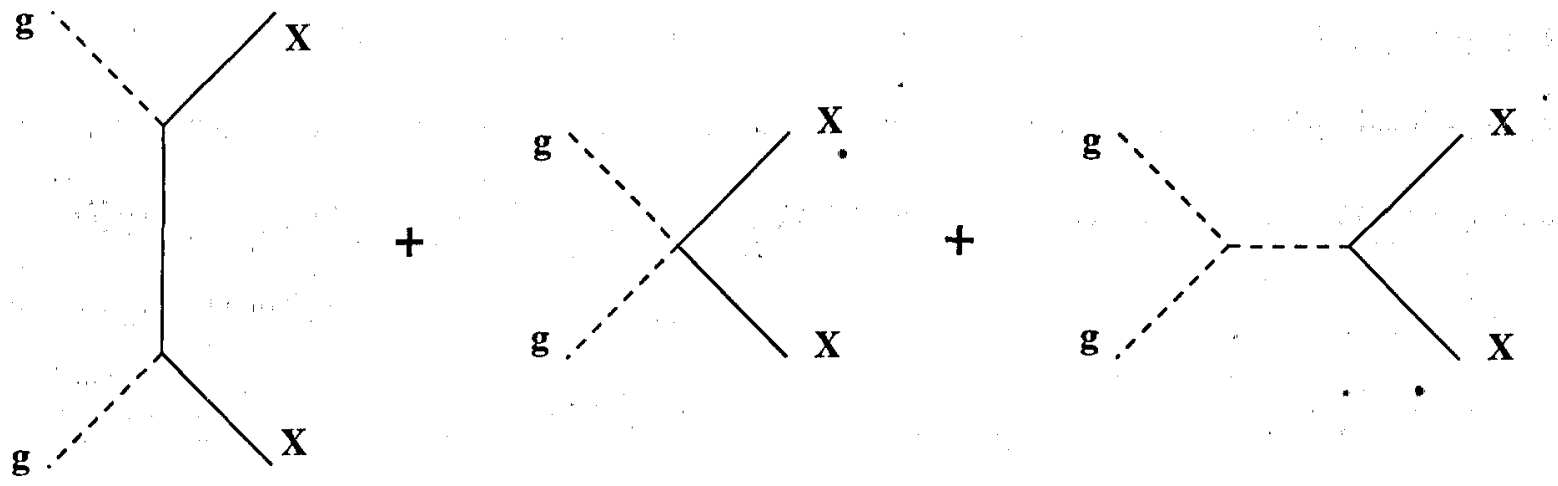

Figure IV.A Contributions to $A^{\text {tree }}(g, g, X, X)$

It is clear that the trees depend only on the external particles involved (ie they are independent of any particular supersymmetric multiplet.) and can be used in a general way.

We saw above that $A\left(g^{+}, g^{+}, \psi^{+}, \psi^{-}\right)=0$, which is the first confirmation of our assertion that gravity does not flip particle helicities at tree level. We can use SUSY identities to show this is true for other particles. For instance, consider repeating (IV.25) with the change $a_{\psi}^{-} \rightarrow a_{\psi}^{+}$. If we note that, at tree level, $\langle 0|g \phi \psi g| 0\rangle=0$ then a similar argument leads to the deduction

$$
A\left(g^{-}, \gamma^{+}, g^{+}, \gamma^{+}\right)=0
$$




\section{Appendix V. Supersymmetric Decomposition}

We find that the most useful way to organise one-loop graviton amplitudes is with a 'SUSY decomposition' inspired by string theory. (For use of this method in QCD and electroweak theories see $[15,24]$.) Rather than calculating amplitudes for individual particles, we do so for certain combinations. In particular, we choose certain SUSY multiplets. Results for any combination of particles required can then be found from the SUSY amplitudes. This method allows us to express a set of results in its most compact form and simplifies the calculations significantly when a suitable calculational technique is used. In the Cutkosky rules, amplitudes containing the larger multiplets can be constrained more strongly than those for individual particles.
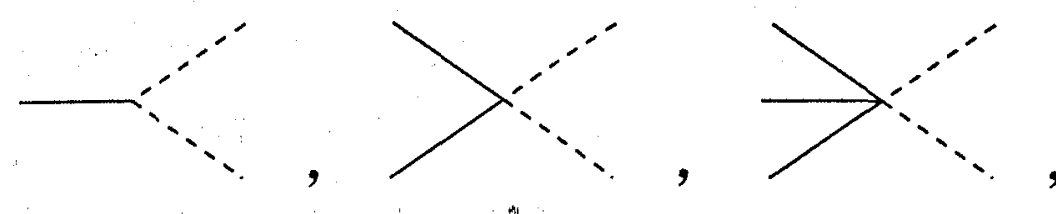

Figure V.A Vertices containing gravitons (solid line) plus other particles (broken line).

To see how such a decomposition is possible, consider that particles coupled to gravitons come in pairs (fig.V.A). So, if we consider one loop diagrams in which the only external particles are gravitons, then we know that they must be constructed from the (one particle irreducible) building blocks in fig.V.B. Clearly, this means that, for this subset of amplitudes, only one particle other than the gravitons can be involved at a time. So, amplitudes involving specific choices of internal particle types are independent from each other.

While we can calculate the contributions from individual particles, the independence of the amplitudes implies that we can calculate linear combinations of the contributions and extract the individual answers at the end. We might hope that, with good choices of the linear combinations, we could reduce the necessary calculations to a small simple set. Since SUSY has simpler results than those from its constituent particles, we might expect the linear combinations of SUSY multiplets to be the most profitable. We find 


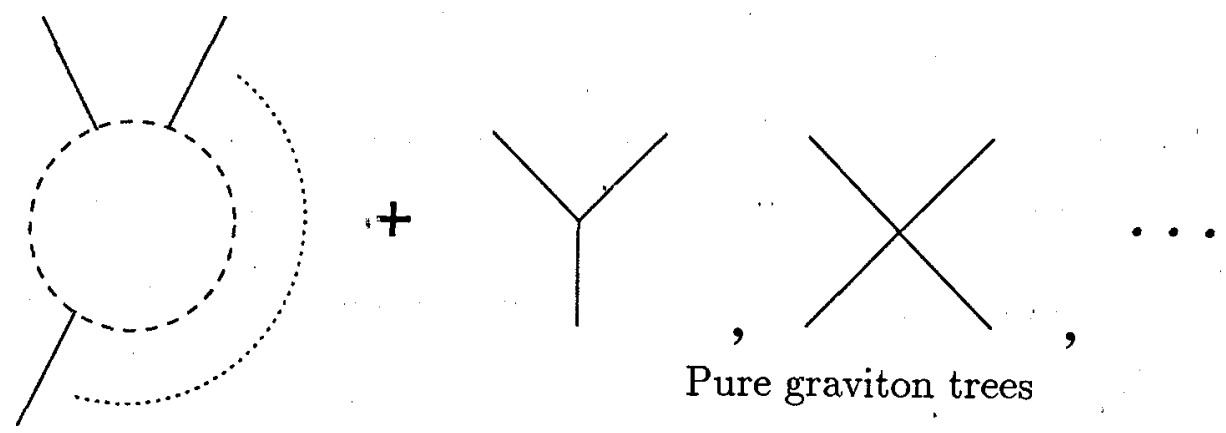

Figure V.B Building blocks for a one-loop graviton amplitude. Gravitons and other particles are represented by solid and broken lines respectively.

in our calculations that this is indeed the case. The multiplets which we choose are the $N=1,4,6,8$ multiplets centred around the scalar. That is, the multiplets in table 5.2. So, in practice we calculate for each of these SUSY multiplets and find expressions for specific individual particles by using

$$
\begin{aligned}
A^{[0]} & =A^{N=0} \\
A^{[1 / 2]} & =A^{N=1}-A^{[0]} \\
A^{[1]} & =A^{N=4}-4 A^{N=1}+A^{[0]} \\
A^{[3 / 2]} & =A^{N=6}-6 A^{N=4}+9 A^{N=1}-A^{[0]} \\
A^{[2]} & =A^{N=8}-8 A^{N=6}+20 A^{N=4}-16 A^{N=1}+A^{[0]}
\end{aligned}
$$

With Feynman diagram methods it is hard to see the advantage of such an approach, since particles of different types are treated differently and Feynman diagrams involving different particles are evaluated separately. However, in both the string-based and Cutkosky methods all particles are treated in essentially the same way and significant simplifications are seen within diagrams when we use this approach. (There are also simplifications seen in superfield calculations when the background field method is employed [91,92].)

In the string-based rules we see the simplifications as cancellations between the common contributions within multiplets. In terms of the notation from chapter 4 and Feynman parameters, we can deduce that for a general $n$-point diagram $S$ there will be a polynomial of degree $n$; the cycle contributions, $C_{V}$ and $C_{F}$ will be of degree $n-2$ and $C_{V}-4 C_{F}$ will 
be of degree $n-4$ (the 2- and 3-cycle contributions will cancel in the combination). For each particle type, there is a scalar contribution $N_{s}[S, S]$ where $N_{s}$ counts the degrees of freedom (with fermions having negative weight). Hence, for any supersymmetric multiplet the $[S, S]$ term will cancel and the Feynman parameter polynomial will be simplified. For higher $N$ supersymmetric multiplets, we see combinations which leave precisely factors of the form $C_{V}-4 C_{F}$ (see table 5.3 for explicit details of this). So, for increasing $N$, the degree of polynomials which appear in calculations decreases and the cycle contributions which must be considered are simplified.

In the Cutkosky method we find that the SU'SY multiplets produce particularly compact expressions, reducing the complexity of the cut calculations. We also find that the uniqueness theorem (see section 6.3) implies that it is the amplitudes for multiplets of higher $\mathrm{N}$, which are most tightly constrained by the cut results. 


\section{Appendix VI. Epsilon Tensor Expansions}

Many of our cut calculations require us to consider traces of the form

$$
\operatorname{tr}_{ \pm}\left(k_{1} k_{2} k_{3} k_{4} \cdots\right)=\frac{1}{2} \operatorname{tr}\left(\left(1+\gamma_{5}\right) k_{1} k_{2} k_{3} k_{4} \cdots\right)
$$

(We often abuse notation and write this as $\operatorname{tr}_{ \pm}(1,2,3,4, \ldots), \operatorname{tr}_{ \pm}\left(k_{1} k_{2} k_{3} k_{4} \cdots\right)$ or some combination of the two.) For a trace of this kind involving four momenta, we can write this as

$$
\operatorname{tr}_{ \pm}\left(k_{1} k_{2} k_{3} k_{4}\right)=\frac{1}{2} \operatorname{tr}\left(k_{1} k_{2} k_{3} k_{4}\right) \pm \frac{1}{2} \epsilon\left(k_{1}, k_{2}, k_{3}, k_{4}\right)
$$

with

$$
\epsilon\left(k_{1}, k_{2}, k_{3}, k_{4}\right)=-4 i \epsilon^{\mu \nu \sigma \rho} k_{1 \mu} k_{2 \nu} k_{3 \sigma} k_{4 \rho}
$$

$\operatorname{The} \operatorname{tr}\left(k_{1} k_{2} k_{3} k_{4}\right)$ is easy to deal with, it becomes [1]

$$
\operatorname{tr}\left(k_{1} k_{2} k_{3} k_{4}\right)=4\left(\left(k_{1} \cdot k_{2}\right)\left(k_{3} \cdot k_{4}\right)-\left(k_{1} \cdot k_{3}\right)\left(k_{2} \cdot k_{4}\right)+\left(k_{1} \cdot k_{4}\right)\left(k_{2} \cdot k_{3}\right)\right)
$$

The $\epsilon$-functions require slightly more work: Note that we are considering amplitudes with four external momenta. These are related by

$$
k_{1}+k_{2}+k_{3}+k_{4}=0
$$

so we only have three independent momenta in the theory. This implies that [17]

$$
\int d^{4} l \epsilon\left(l, k_{i}, k_{j}, k_{m}\right)=0
$$

More generally,

$$
\int d^{4} l \epsilon\left(l, k_{i}, k_{j}, k_{m}\right) f\left(l, k_{1}, k_{2}, k_{3}, k_{4}\right)=0
$$

unless $f$ contains something which will alter the $\epsilon\left(l, k_{i}, k_{j}, k_{m}\right)$. In fact, another $\epsilon$-function will do this, since products of $\epsilon$-tensors produce sums of Kronecker deltas. So, we can discard products of odd numbers of $\epsilon$-functions. We will expand a product of an even 
number as dot-products of momenta. To enable us to do this, we list here explicit results for the products of $\epsilon$-functions required in the text.

A product of $2 \epsilon$-functions can be written explicitly as

$$
\epsilon(a, b, c, d) \epsilon(e, f, g, h)=-16 \epsilon^{\mu \nu \rho \sigma} \epsilon^{\mu^{\prime} \nu^{\prime} \rho^{\prime} \sigma^{\prime}} a_{\mu} b_{\nu} c_{\rho} \dot{d}_{\sigma} e_{\mu^{\prime}} f_{\nu^{\prime}} g_{\rho^{\prime}} h_{\sigma^{\prime}}
$$

So,we can use [1]

$$
\begin{aligned}
\epsilon^{\mu \nu \rho \sigma} \epsilon^{\mu^{\prime} \nu^{\prime} \rho^{\prime} \sigma^{\prime}}=-\operatorname{det}\left(\eta_{\alpha \alpha^{\prime}}\right) \cdot \quad & \alpha=\mu, \nu, \rho, \sigma \\
\therefore \quad & \alpha=\mu^{\prime}, \nu^{\prime}, \rho^{\prime}, \sigma^{\prime}
\end{aligned}
$$

to rewrite this as an expression consisting only of dot products. Obviously this will be a large and complicated expression in general, but if some of the momenta are shared between the epsilon functions and are on-shell, it is possible to obtain surprisingly compact answers.

Consider first the case with two shared on-shell momenta

$$
\epsilon\left(k_{1}, k_{2}, k_{3}, k_{4}\right) \epsilon\left(p_{1}, p_{2}, k_{3}, k_{4}\right)
$$

with $k_{3}^{3}=0$ and $k_{4}^{2}=0$.

We find this to be

$$
\begin{aligned}
& 16\left(-(k 1 . p 1)(k 2 . p 2)(k 3 . k 4)^{2}+(k 1 . p 2)(p 1 . k 2)(k 3 . k 4)^{2}\right. \\
&+(k 1 . p 1)(k 2 . k 4)(p 2 . k 3)(k 3 . k 4)-(k 1 . k 4)(p 1 . k 2)(p 2 . k 3)(k 3 . k 4) \\
&-(k 1 . k 3)(p 1 . k 2)(p 2 . k 4)(k 3 . k 4)-(k 1 . p 2)(p 1 . k 3)(k 2 . k 4)(k 3 . k 4) \\
&+(k 1 . k 4)(p 1 . k 3)(p 2 . k 2)(k 3 . k 4)+(k 1 . k 3)(p 1 . k 3)(k 2 . k 4)(p 2 . k 4) \\
&-(k 1 . k 4)(p 1 . k 3)(k 2 . k 3)(p 2 . k 4)-(k 1 . p 2)(p 1 . k 4)(k 2 . k 3)(k 3 . k 4) \\
&+(k 1 . k 3)(p 1 . k 4)(k 2 . p 2)(k 3 . k 4)-(k 1 . k 3)(p 1 . k 4)(k 2 . k 4)(p 2 . k 3) \\
&+(k 1 . p 1)(k 2 . k 3)(p 2 . k 4)(k 3 . k 4)+(k 1 . k 4)(p 1 . k 4)(k 2 . k 3)(k 3 . p 2))
\end{aligned}
$$


Similarly,

$$
\begin{aligned}
& \epsilon\left(k_{1}, k_{2}, k_{3}, k_{4}\right) \epsilon\left(p_{1}, k_{2}, k_{3}, k_{4}\right)= \\
& 16\left(2(k 1 . p 1)(k 2 . k 4)(k 2 . k 3)(k 3 . k 4)+(k 1 . k 2)(p 1 . k 2)(k 3 . k 4)^{2}\right. \\
&-(k 1 . k 4)(p 1 . k 2)(k 2 . k 3)(k 3 . k 4)-(k 1 . k 3)(p 1 . k 2)(k 2 . k 4)(k 3 . k 4) \\
&-(k 1 . k 2)(p 1 . k 3)(k 2 . k 4)(k 3 . k 4)+(k 1 . k 3)(p 1 . k 3)(k 2 . k 4)^{2} \\
&-(k 1 . k 4)(p 1 . k 3)(k 2 . k 3)(k 2 . k 4)-(k 1 . k 2)(p 1 . k 4)(k 2 . k 3)(k 3 . k 4) \\
&-(k 1 . k 3)(p 1 . k 4)(k 2 . k 4)(k 2 . k 3)+(k 1 . k 4)(p 1 . k 4)(k 2 . k 3)(k 3 . k 2))
\end{aligned}
$$

Obviously, the simplest form comes from the case where all momenta are shared and on-shell:

$$
\begin{aligned}
& \epsilon\left(k_{1}, k_{2}, k_{3}, k_{4}\right) \epsilon\left(k_{1}, k_{2}, k_{3}, k_{4}\right) \\
& =16\left((k 1 . k 2)^{2}(k 3 . k 4)^{2}-2(k 1 . k 4)(k 1 . k 2)(k 2 . k 3)(k 3 . k 4)\right. \\
& +(k 1 . k 3)^{2}(k 2 . k 4)^{2}-2(k 1 . k 3)(k 1 . k 2)(k 2 . k 4)(k 3 . k 4) \\
& \left.+(k 1 . k 4)^{2}(k 2 . k 3)^{2}-2(k 1 . k 4)(k 1 . k 3)(k 2 . k 4)(k 2 . k 3)\right) .
\end{aligned}
$$




\section{References}

[1] C. Itzykson and J-B Zuber, Quantum Field Theory (McGraw-Hill Book Co., 1980).

[2] M.L. Mangano and S.J. Párke, Phys. Rep. 200:301 (1991).

[3] Z. Bern and D.A. Kosower, Phys. Rev. Lett. 66:1669 (1991); Nucl. Phys. B379:451 (1992).

[4] Z. Bern and D.A. Kosower, in Proceedings of the PASCOS-91 Symposium, eds. P. Nath and S. Reucroft (World Scientific, 1992).

[5] L.D. Landau, Nucl. Phys. 13:181 (1959);

S. Mandelstam, Phys. Rev. 112:1344 (1958), 115:1741 (1959);

R.E. Cutkosky, J. Math. Phys. 1:429 (1960).

[6] M.J. Strassler, Nucl. Phys. B385:145 (1992), SLAC-PUB-5978 (1992);

M.G. Schmidt and C. Schubert, Phys. Lett. 318B:438 (1993);

D. Cangami, E. D'Hoker and G. Dunne, Phys Rev D51:2513 (1995);

M.G. Schmidt and C. Schubert, hep-ph/9412358.

[7] M.G. Schmidt and C. Schubert, Phys. Lett. 331B:69 (1994);

C.S. Lam, hep-ph/9406388.

[8] G.D. Mahlon, Phys. Rev. D49:2197 (1994), Phys. Rev. D49:4438 (1994).

[9] Z. Bern, G. Chalmers, L. Dixon, and D. A. Kosower, Phys. Rev. Lett. $72: 2134$ (1994).

[10] Z. Bern, D.C. Dunbar, L. Dixon and D.A. Kosower, Nucl. Phys. B425:217 (1994).

[11] Z. Bern and G. Chalmers, Nucl. Phys. B447:465 (1995).

[12] J. Scherk, Nucl. Phys. B31:222 (1971);

A. Neveu and J. Scherk, Nucl. Phys. B36:155 (1972).

[13] J. Minahan, Nucl. Phys. B298:36 (1988);

I. Senda, Phys. Lett. 206B:473 (1988);

K. Miki, Nucl. Phys. B291:349 (1987);

M.B. Green and N. Seiberg, Nucl. Phys. B299:559 (1988).

[14] Z. Bern, Phys. Lett. 296B:85 (1992). 
[15] Z. Bern, in Proceedings of Theoretical Advanced Study Institute in High Energy Physics (TASI 92), eds. J. Harvey and J. Polchinski (World Scientific, 1993) (hep-ph/9304249).

[16] R.J. Eden, P.V. Landshoff; D.I. Olive and J.C. Polkinghorne, The Analytic S-Matrix (Cambridge University Press, 1966).

[17] Z. Bern, D.C. Dunbar, L. Dixon and D.A. Kosower, Nucl..Phys. B435:59 (1995)."

[18] Z. Bern and A. Morgan, hep-ph/9511336.

[19] P. De Causmaeker, R. Gastmans, W. Troost and T.T. Wu, Nucl. Phys. B206:53 (1982); R. Kleiss and W. J. Stirling, Nucl. Phys. B262:235 (1985);

J. F. Gunion and Z. Kunszt, Phys. Lett. 161ن:333 (1985);

R. Gastmans and T.T. Wu, The Ubiquitous Photon: Helicity Method for QED and QCD (Clarendon Press) (1990);

Z. Xu, D.-H. Zhang and L. Chang, Nucl. Phys. B291:392 (1987).

[20] F.A. Berends, W.T. Giele and H. Kuijf, Phys. Lett. 211B:91 (1988).

[21] S.F.Novaes and D.Spehler, Phýs.Rev. D44:3990 (1991); Nucl.Phys. B371:618 (1992); H.T. Cho, K.L. Ng, Phys. Rev. D47:1692 (1993) : .

[22] M.T. Grisaru, H.N. Pendleton and P. van Nieuwenhuizen, Phys. Rev. D15:996 (1977); M.T. Grisaru and H.N. Pendleton, Nucl. Phys. B124:81 (1977).

[23] S.J. Parke and T.R. Taylor, Phys.Lett.157B:81 (1985) [Erratum: 174B:465 (1986)].

[24] Z. Bern and A. Morgan, Phys. Rev. D49:6155 (1994).

[25] Z. Bern, L. Dixon and D.A. Kosower, Phys. Rev. Lett. 70:2677 (1993); DPF Conf.1992:901-905 (hep-ph/9212237) .

[26] Z. Bern, L. Dixon, D. Dunbar, and D.A. Kosower, hep-ph/9405248.

[27] Z. Bern, L. Dixon and D.A. Kosower, Nucl. Phys. B437:259 (1995).

[28] B.S. DeWitt, Phys. Rev. 162:1239 (1967).

[29] M. Veltman, in Les Houches 1975, Methods in Field Theory, ed R. Balian and J. Zinn-Justin, (North Holland, Amsterdam, 1976);

R.P. Feynman, Lectures on Gravitation (Addison-Wesley, 1995). 
[30] P. van Nieuwenhuizen, Phys. Rep. 68:189 (1981).

[31] Z. Bern, D.C. Dunbar and T. Shimada, Phys. Lett. 312B:277 (1993).

[32] S. Weinberg, Gravitation and Cosmology (Wiley, 1972).

[33] Quantum Gravity, eds. C. Isham, R. Penrose and D. Sciama (Oxford, 1975).

[34] M. B. Green, J.H. Schwarz, and E. Witten, Superstring Theory (Cambridge University Press, 1987).

[35] R. Utiyama and B.S. DeWitt, J. Math. Phys. 3:608 (1962).

[36] S. Weinberg, in General Relativity: An Einstein Centenary Survey, eds. S.W. Hawking and W. Israel (Cambridge University Press, 1979).

[37] P. West, Introduction to Supersymmetry and Supergravity (World Scientific, 1992).

[38] S. Mandelstam, in Miami 1983, Proceedings, High-energy Physics (1987). .

[39] J.F. Donoghue, gr-qc/9512024.

[40] S. Weinberg, Phys. Rev. 140:B516 (1965).

[41] H. Weyl, Z. Phys. 56:330 (1929); Phys. Rev. 77:699 (1950).

[42] S. Deser and P. van Nieuwenhuizen, Phys. Rev. D10:411 (1974).

[43] R.P. Woodard, Phys. Lett. 148B:440 (1984).

[44] W. Rarita and J. Schwinger, Phys. Rev. 60:61 (1941).

[45] S. Ferrara, D.Z. Freedman and P. van Nieuwenhuizen, Phys. Rev. D14:912 (1976).

[46] P.D.B. Collins, A.D. Martin and E.J. Squires in Particle Physics and Cosmology (Wiley, 1989).

[47] S. Deser and B. Zumino, Phys. Lett. 62B:335 (1976).

[48] S. Ferrara, P. van Nieuwenhuizen and J. Scherk, Phys. Rev. Lett. 37:1035 (1976);

S. Ferrara, P. van Nieuwenhuizen, P. Breitenlohner, F. Gliozzi and J. Scherk, Nucl. Phys. B117:333 (1977);

S. Ferrara, D.Z. Freedman, P. van Nieuwenhuizen, P. Breitenlohner, F. Gliozzi and J. Scherk, Phys. Rev. D15:1013 (1977).

[49] S. Ferrara and P. van Nieuwenhuizen, Phys. Rev. Lett. 37:1669 (1976). 
[50] D.Z. Freedman, Phys. Rev. Lett. 38:105 (1977);

S. Ferrara, J. Scherk and B. Zumino, Phys. Lett. 66B:35 (1977).

[51] A. Das, Phys Rev D15:2805 (1977);

E. Cremmer and J. Scherk, Phys. Lett. 69B:97 (1977), Nucl. Phys. B127:259 (1977);

E. Cremmer, S. Ferrara and J. Scherk, Phys. Lett. 74B:404 (1978).

[52] B. de Wit, F.A. Berends, •J.W. van Holten and P. van Nieuwenhuizen, Phys. Lett. 83B:188 (1979), Nucl. Phys. B154:261 (1979).

[53] D.Z. Freedman and B. de Wit, Nucl. Phys. B130:105 (1977);

E. Cremmer and B. Julia, Phys. Lett. 80B:48 (1979), Nucl. Phys. B159:141 (1979).

[54] S. Y. Choi, J. S. Shim, and H.. S. Song, hep-th/9411092.

[55] G. 't Hooft, Acta Universitatis Wratislavensis no. 38, 12th Winter School of Theoretical Physics in Karpacz; Functional and Probabilistic Methods in Quantum Field Theory, Vol. 1 (1975);

B.S. DeWitt, in Quantum Gravity II, eds. C. Isham, R. Penrose and D. Sciama (Oxford, 1981);

L.F. Abbott, Nucl. Phys. B185:189 (1981);

L.F Abbott, M.T. Grisaru and R.K. Schaeffer, Nucl. Phys. B229:372 (1983).

[56] G. 't Hooft and M. Veltman, Ann. Inst. Henri Poincaré 20:69 (1974).

[57] L.F. Abbott, M.T. Grisaru and R.K. Shaefer, Nucl. Phys. B229:372 (1983).

[58] Z. Bern and D.C. Dunbar, Nucl. Phys. B379:562 (1992).

[59] L.L. Gervais and A. Neveu, Nucl. Phys. B46:381 (1972).

[60] S. Sannan, Phys. Rev. D34:1748 (1986).

[61] H. Kawai, D.C. Lewellen and S.H.H. Tye, Nucl. Phys. B269:1 (1986).

[62] D.M. Capper and G. Leibbrandt, Nuovo Cimento Lett. 6:117 (1973);

D.M. Capper, G. Leibbrandt and M. Ramón Medrano, Phys. Rev. D8:4320 (1973);

D.M. Capper and M.J. Duff, Nuovo Cimento A 23:173 (1974);

D.M. Capper and M.J. Duff, Nucl. Phys. B82:147 (1974); 
D.M. Capper and G. Leibbrandt, J. Math. Phys. 15:795 (1974) .

[63] D.M. Capper, M.J. Duff and L. Halpern Phys. Rev. D10:461 (1974).

[64] M.T. Grisaru and J. Zak, Phys. Lett. 90B:237 (1980).

[65] M.B. Green, J.H. Schwarz and L. Brink, Nucl. Phys. B198:472 (1982).

[66] J. Collins, Renormalization (Cambridge University Press, 1984).

[67] T. Kinoshita, J. Math. Phys. 3:650 (1962);

T.D. Lee and M. Nauenberg, Phys. Rev. 133B:1549 (1964);

T. Kinoshita and A. Ukawa, Phys. Rev. D13:1573 (1976).

[68] G. 't Hooft and M. Veltman, Nucl. Phys. B44:189 (1972);

C.G. Bollini and J.J. Giambiagi, Phys. Lett. 40B:566 (1972); Nuovo Cimento 12B:189 (1972);

J.F. Ashmore, Nuovo Cimento Lett. 4:289 (1972); Comm. Math. Phys. 29:177 (1973);

G. Leibbrandt, Rev. Mod. Phys. 47:849 (1975).

[69] W. Siegel, Phys. Lett. 84B:193 (1979);

D.M. Capper, D.R.T. Jones and P. van Nieuwenhuizen, Nucl. Phys. B167:479 (1980);

L.V. Avdeev and A.A. Vladimirov, Nucl. Phys. B219:262 (1983);

I. Jack, D.R.T. Jones and K.L. Roberts, Z. Phys. C63:151 (1994).

[70] G. 't Hooft, Nucl. Phys. B62:444 (1973).

[71] S. Deser and P. van Nieuwenhuizen, Phys. Rev. D10:401 (1974);

M.T. Grisaru, P. van Nieuwenhuizen and C.C. Wu, Phys. Rev. D12:1813 (1975).

[72] S. Deser, Hung-Sheng Tsao and P, van Nieuwenhuizen, Phys. Lett. 50B:491 (1974); Phys. Rev. D10:3337 (1974).

[73] M.T. Grisaru, P. van Nieuwenhuizen and J.A.M. Vermaseren, Phys. Rev. Lett. 25:1662 (1976).

[74] M.H. Goroff and A. Sagnotti, Phys. Lett. 150B:81 (1985); Nucl. Phys. B266:709 (1986);

A.E.M. van de Ven, Nucl. Phys. B378:309 (1992).

[75] P. van Nieuwenhuizen and J.A.M. Vermaseren, Phys. Rev. D16:298 (1977). 
[76] M.T. Grisaru, Phys. Lett. 66B:75 (1977).

[77] P. van Niewenhuizen and C.C. Wu, J. Math. Phys. 18:81 (1977).

[78] S. Deser, J.H. Kay and K.S. Stelle, Phys. Rev. Lett. 38:527 (1977).

[79] Z. Bern and D.A. Kosower, Nucl. Phys. B362:389 (1991).

[80] J.E. Paton and H.M. Chan, Nucl. Phys. B10:516 (1969).

[81] Z. Bern and D.A. Kosower, Phys. Rev. D38:1888 (1988);

Z. Bern, D.A. Kosower and K. Roland, Nucl. Phys. B334:309 (1990).

[82] E. Cremmer and J. Scherk, Nucl. Phys. B103:399 (1976).

[83] Z. Bern, L. Dixon and D.A. Kosower, Phys. Lètt. 302B:299 (1993) [Erratum: B318:649 (1993)]; Nucl. Phys. 412:752 (1994) .

[84] D. Gross and R. Jackiw, Phys. Rev. 166:1287 (1968).

[85] L.M. Brown and R.P. Feynman, Phys. Rev. 85:231 (1952);

G. Passarino and M. Veltman, Nucl. Phys. B160:151 (1979);

G. 't Hooft and M. Veltman, Nucl. Phys. B153:365 (1979);

R.G. Stuart, Comput. Phys. Comm. 48:367 (1988);

R.G. Stuart and A. Gongora, Comput. Phys. Comm. 56:337 (1990).

[86] Y.Q. Cai and G. Papini, Phys. Rev. Lett.66:1259 (1991);

R. Aldrovandi, G.E.A. Matsas, S.F. Novaes and D. Spehler, Phys. Rev. D50:2645 (1994).

[87] L. Dixon, Private Communication.

[88] R.Aldrovandi, S.F.Novaes and D.Spehler, Gen. Rel. Grav. 26:409 (1994).

[89] K. Roland, Phys. Lett. 289B:148 (1992); .

G. Cristofano, R. Marotta and K. Roland, Nucl. Phys. B 392:345 (1993).

[90] D.C. Dunbar and P.S. Norridge, Nucl. Phys. B433:181 (1995).

[91] M.T. Grisaru and W. Siegel, Nucl. Phys. B187:149 (1981).

[92] S.J. Gates, M.T. Grisaru, M. Rocek and W. Siegel, Superspace, (Benjamin/Cummings, 1983). 